\title{
Differences in Sexual Delay Discounting Among In-Treatment Adults with Opioid Use Disorder
}

\author{
Jonathan J.K. Stoltman \\ West Virginia University, jonathan.stoltman@gmail.com
}

Follow this and additional works at: https://researchrepository.wvu.edu/etd

Part of the Behavioral Economics Commons, Behavioral Medicine Commons, Developmental

Psychology Commons, Health Psychology Commons, and the Substance Abuse and Addiction Commons

\section{Recommended Citation}

Stoltman, Jonathan J.K., "Differences in Sexual Delay Discounting Among In-Treatment Adults with Opioid Use Disorder" (2019). Graduate Theses, Dissertations, and Problem Reports. 4071.

https://researchrepository.wvu.edu/etd/4071

This Dissertation is protected by copyright and/or related rights. It has been brought to you by the The Research Repository @ WVU with permission from the rights-holder(s). You are free to use this Dissertation in any way that is permitted by the copyright and related rights legislation that applies to your use. For other uses you must obtain permission from the rights-holder(s) directly, unless additional rights are indicated by a Creative Commons license in the record and/ or on the work itself. This Dissertation has been accepted for inclusion in WVU Graduate Theses, Dissertations, and Problem Reports collection by an authorized administrator of The Research Repository @ WVU.

For more information, please contact researchrepository@mail.wvu.edu. 
Differences in Sexual Delay Discounting Among In-Treatment Adults with Opioid Use Disorder

Jonathan J.K. Stoltman, M.A., M.S.

\author{
Dissertation submitted to the \\ Eberly College of Arts and Sciences \\ at West Virginia University \\ in partial fulfillment of the requirements for the degree of \\ Doctor of Philosophy in \\ Psychology
}

Julie Hicks Patrick, Ph.D., Chair

Karen Anderson, Ph.D.

Aaron Metzger, Ph.D.

Robin Pollini, Ph.D.

Department of Psychology

Morgantown, West Virginia

2019

Keywords: Delay-discounting, Sexual decision-making, Individual differences

Copyright 2019, Jonathan Joseph Kelly Stoltman 


\section{Abstract \\ Differences in Sexual Delay Discounting Among In-Treatment Adults with Opioid Use}

\section{Disorder}

\section{Jonathan J.K. Stoltman}

Previous research has found impulsive decision-making to be a core component of addiction (Moody, Franck, Hatz, \& Bickel, 2016). One way to measure impulsive choice is through the use of a delay discounting task. The delay discounting task provides a way to measure choice of immediate, smaller rewards compared to delayed, larger rewards (Odum, 2011b). An emerging area of research in addiction science is the intersection of addiction and sexual health. Previous sexual delay discounting research has focused on whether attractiveness or STD risk can shift the likelihood of waiting until a condom was available (Johnson \& Bruner, 2012). This study is among the first to include a pregnancy-risk condition, utilize shorter delays, and include individual difference variables such as gender, future time perspective, condom attitudes, and sexual arousal. The final analysis included 113 adults in treatment for Opioid Use Disorder. Two monetary discounting conditions $(\$ 10, \$ 100)$ and three sexual discounting conditions (attraction, STD risk, pregnancy risk) were used to test differences in choice between and within condition. Results indicated significant differences between and within monetary and sexual domains. Next, magnitude differences were observed within each monetary and sexual condition. Lastly, individual differences indicated participants with higher views of the Future as Limited and Future as Ambiguous were less likely to wait for a delayed condom in the Low Pregnancy Risk condition. Higher condom pleasure was associated with a greater likelihood of waiting until a delayed condom was available in the High Attraction, Low STD Risk, and Low Pregnancy Risk conditions. This study advances our understanding of impulsive decision-making and addiction. First, between condition differences were observed indicating that monetary and sexual decision making are distinct concepts that can yield distinct patterns of behavior. The High Attraction condition and the Low STD Risk condition had the least likelihood of waiting until a condom was available. Interestingly, the Low Pregnancy Risk condition elicited a greater likelihood of waiting until a condom was available. This suggests that STD Risk and Pregnancy Risk are evaluated differently and could motivate decision-making. Second, within condition differences were observed indicating that magnitude or level of risk can shift responding, even with the use of hypothetical constructs. Third, individual differences variables such as future time perspective and condom attitudes are associated with delayed condom choice. 


\section{Acknowledgements}

My time as a Mountaineer has provided me with an opportunity to work with many amazing people. There are too many to name, but I will try. This study could not have happened without the patients at the COAT clinic. My questions for them were sensitive, and their time is important. Without their commitment and interest in research, this project would not have been possible. Thank you to Julie Hicks Patrick, Ph.D. Her guidance has helped me throughout my doctoral training. The lessons that Dr. Patrick has taught me will help to carry me through both personal and professional challenges. I appreciate the time and comments provided by my committee members, Karen Anderson, Ph.D., Aaron Metzger, Ph.D., and Robin Pollini, Ph.D. This document and my ability to tackle large projects are improved, having worked with each of you. Laura Lander MSW, thank you for your tremendous support through all phases of this project and for your continued advocacy to advance sexual health services in the COAT clinic. Thank you, Matthew W. Johnson, Ph.D. for providing the Qualtrics codebook to capture the delay discounting data and the intellectual support during the design of this project. Thank you to Hendrée Jones, Ph.D. and Mishka Terplan, M.D. for inspiring me to pursue this path at the intersection of addiction and sexual health and for setting out a blueprint for how to do so with through your tremendous and impactful careers. Thank you to the COAT clinic group-leaders who provided instrumental support throughout the project. A special thank you to the administrators at Ruby Memorial Hospital's Chestnut Ridge Center, in particular, Rolly Sullivan, M.D. and Jim Berry D.O. who laid the groundwork for this project. Thank you to the WVCTSI for their financial and logistical support. A special thank you to Valerie Blake and Tanisha Hendrix. Without you, much of this data could not have been collected. You allowed me to be in two places at once and did so with class and grace.

I am thankful to my mother, Mary Pat Stoltman, and father, Jeffrey Stoltman for all their trips and calls to the mountain to show their support. You were my secret weapons throughout this process and always understood the what and why of my time here. I am thankful for my family's support and encouragement throughout this whole process: Joan and Todd, Lindsey and Lee, Ben and Annie, Caiti and Reed, Gordie Quentin, grandmas and grandpa, aunts and uncles, and cousins who always asked and cared about my progress. Thank you to my friends and family for their well-timed trips to the mountains where they reminded me to get outside and enjoy all that wild and wonderful West Virginia has to offer.

A special thank you to Ellen Manegold, who in equal measure inspired and celebrated my accomplishments throughout my training.

Montani Semper Liberi 


\section{Table of Contents}

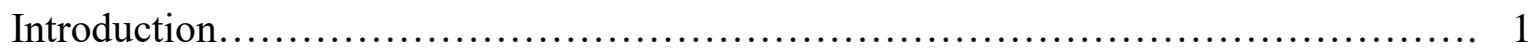

Delay Discounting.................................................. 1

Sexual Discounting Task.............................................. 4

Domains, Delay Length, Reward Magnitude, and Individual Differences............. 4

Life-span Specific Considerations..................................... 6

Sexual Intercourse-specific Considerations............................. 8

The Opioid Epidemic.................................................... 9

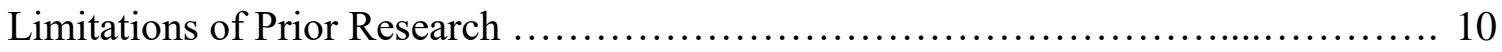

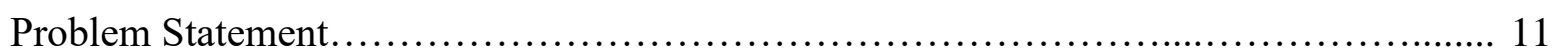

Research Questions and Hypotheses.......................................... 12

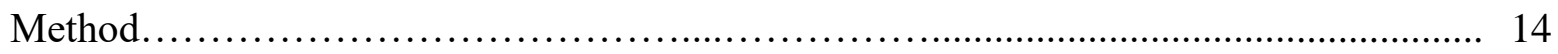

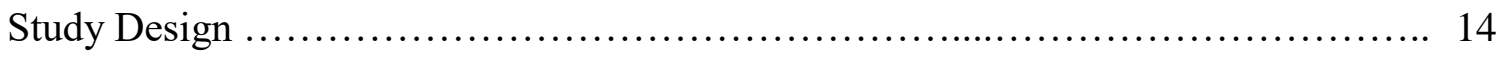

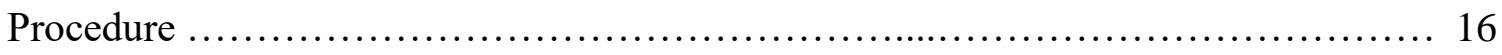

Survey Software................................................. 17

Measures..................................................................... 18

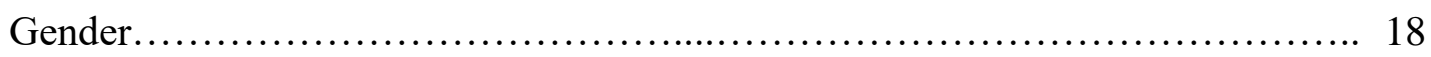

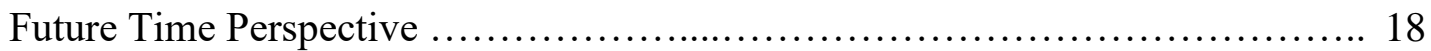

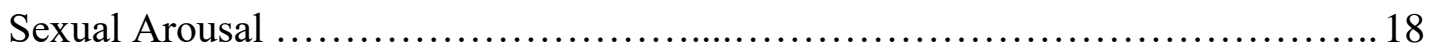

Condom Attitudes.................................................... 19

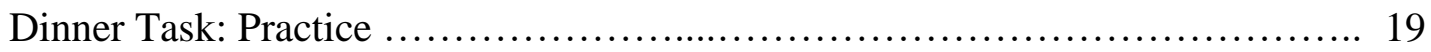

Monetary Delay-Discounting Task ........................................ 19

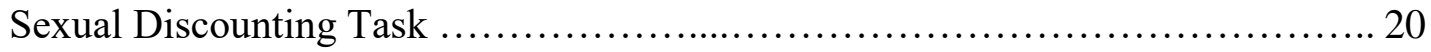


Sexual Discounting Task: Attractiveness.............................. 22

Sexual Discounting Task: STD Risk.................................... 23

Sexual Discounting Task: Pregnancy Risk.............................2 24

Data Processing.................................................................. 24

Delay Discounting Data Cleaning................................. 25

Delay discounting Data Coding..................................... 27

Monetary Delay-Discounting Task AUC Formula................. 28

Sexual Discounting Task AUC Formula....................... 28

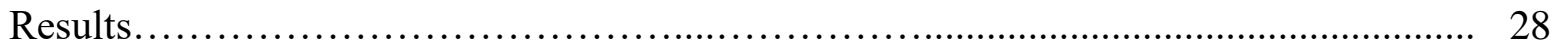

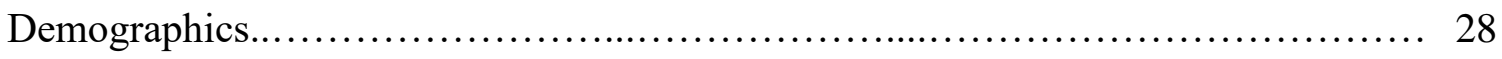

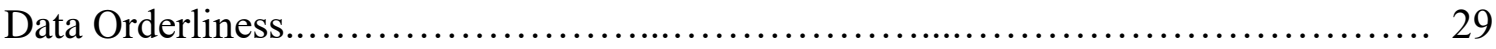

Data Normality............................................................ 30

Non-systematic Data........................................................ 30

Order Effects............................................................. 31

The Analytic Sample........................................................ 32

Data External Validity Check .................................................... 32

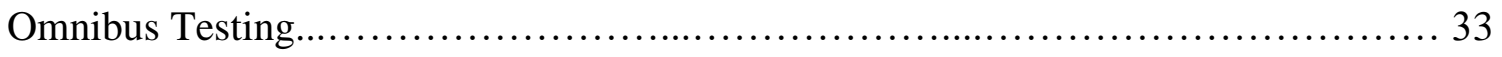

Research Question 1: Are there domain differences in delay discounting?............. 33

Research Question 2: Does reward magnitude influence choice for monetary and sexual discounting?

Research Question 3: Are differences in sexual discounting associate with

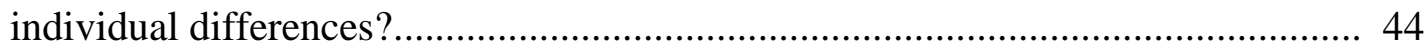

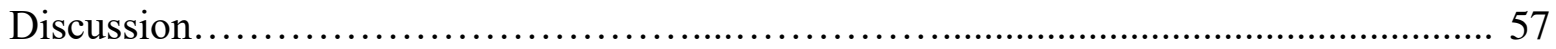


Domain Differences between Delay Discounting AUCs......................... 58

Condition Differences between Delay Discounting AUCs.......................... 61

Reward Magnitude Differences........................................... 62

Individual Differences and Sexual Discounting................................. 64

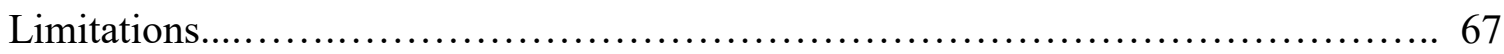

Future Directions and Conclusions........................................... 70

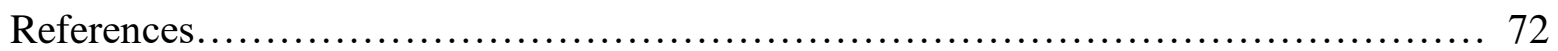

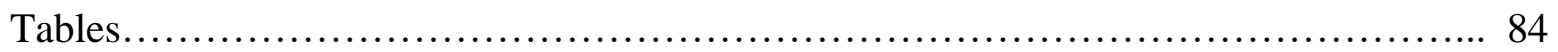

Figures................................................................ 98

Appendices............................................................. 104

Appendix A: Consent form and all measures used in the study..................... 104

Appendix B: Procedural details ............................................. 204

Appendix C: Randomization procedures...................................... 206

Appendix D: Monetary Delay Discounting Task and Sexual Discounting Task AUC

Formula........................................................... 207

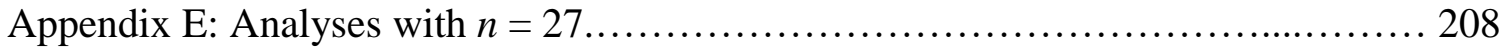

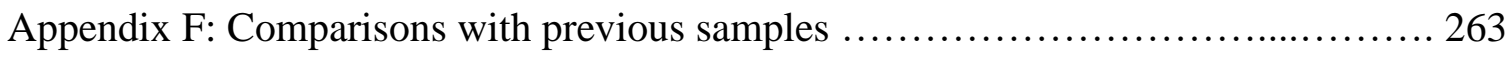




\section{List of Tables}

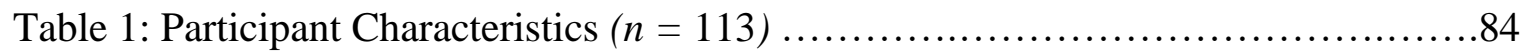

Table 2: Descriptive Statistics for AUC Using Imputed Data $(n=113) \ldots \ldots \ldots \ldots \ldots . \ldots . \ldots 5$

Table 3: Dependent Samples Wilcoxon Signed-ranks Test Median Differences

Between and Within Conditions using Imputed AUC Data $(n=113) \ldots \ldots \ldots \ldots . . . .86$

Table 4: Spearman Correlation Coefficient with Raw AUC Using Pooled

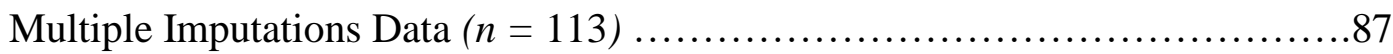

Table 5: Mann-Whitney U Comparing AUC and Median Split Future as

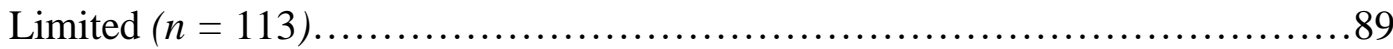

Table 6: Mann-Whitney U Comparing AUC and Median Split Future as

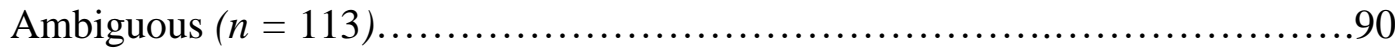

Table 7: Mann-Whitney U Comparing AUC and Median Split MCAS

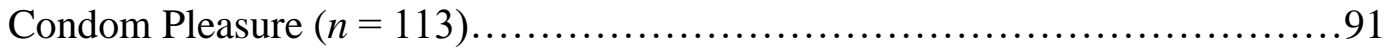

Table 8: Mann-Whitney U Comparing AUC and Median Split MCAS

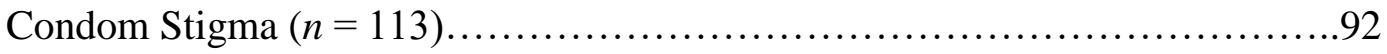

Table 9: Mann-Whitney U Comparing AUC and Median Split MCAS Embarrassment

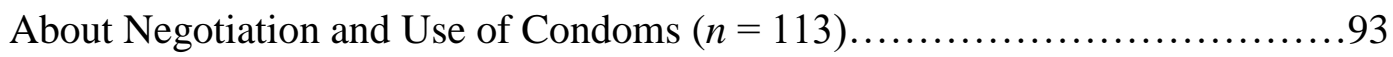

Table 10: Mann-Whitney U Comparing AUC and Median Split Sexual

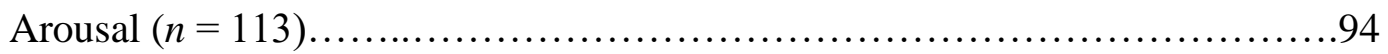

Table 11: Mann-Whitney U Comparing AUC and Gender $(n=113) \ldots \ldots \ldots \ldots \ldots \ldots . \ldots 9$

Table 12: Mann-Whitney U Comparing AUC and Reproductive-aged

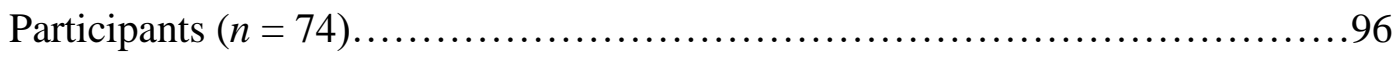

Table 13: Mann-Whitney U Comparing AUC and Relationship Status $(n=113) \ldots \ldots . . .97$ 


\section{Appendices..........................................................................104}

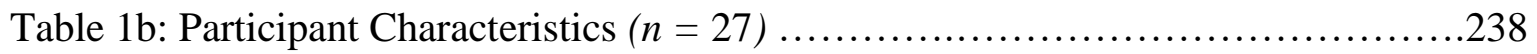

Table 2b: Descriptive Statistics for Raw AUC, Standardized AUC, and

“Immediately Available" Condom Preferences $(n=27)$

Table 3b: Dependent Samples Wilcoxon Signed-ranks Test Median Differences

Between and Within Condition using Raw AUC, Standardized AUC, and "Immediately Available" Condom Preference $(n=27) \ldots \ldots \ldots \ldots \ldots \ldots \ldots . . .240$

Table 4b: Spearman Correlation Coefficient with Raw AUC $(n=27) \ldots \ldots \ldots \ldots \ldots . . . .241$

Table 5b: Spearman Correlation Coefficient with Standardized AUC $(n=27) \ldots \ldots \ldots \ldots \ldots . . .243$

Table 6b: Spearman Correlation Coefficient with "Immediately Available"

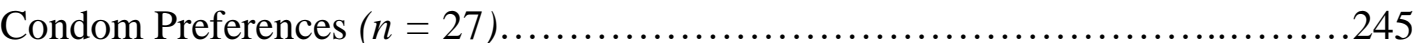

Table 7b: Mann-Whitney U Comparing Gender and Sexual Discounting $(n=27) \ldots \ldots . .247$

Table 8b: Mann-Whitney U Comparison of Participants with Imputed Data to those

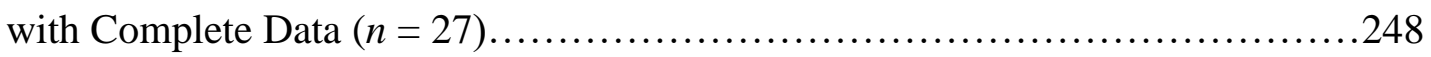




\section{List of Figures}

Figure 1. Sexual Discounting Task data orderliness ..................................98

Figure 2. Monetary Delay-Discounting Task data orderliness........................ 99

Figure 3. Illustrating the computation of AUC using sample data...................... 100

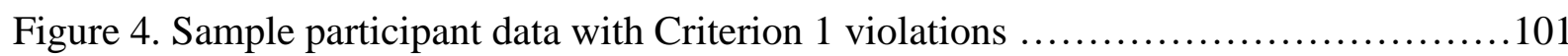

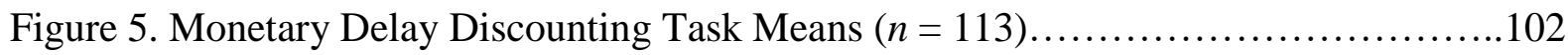

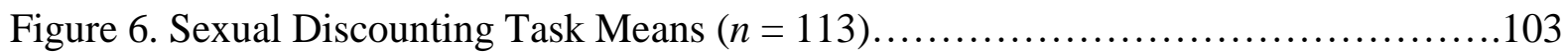

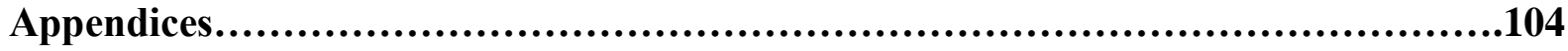

Figure 1b. Percent of Immediate Value Chosen by Delay Using Complete

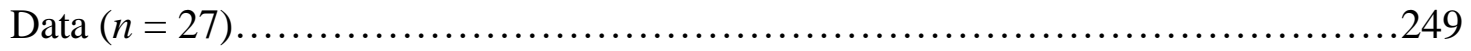

Figure 2b. Percent Likelihood of Condom Use by Delay Using

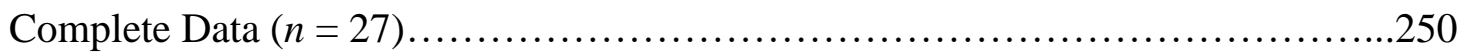

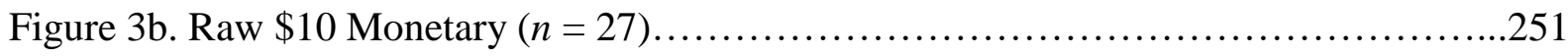

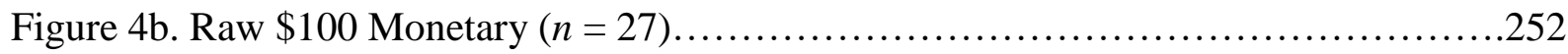

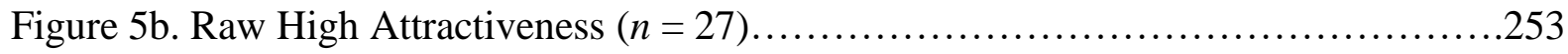

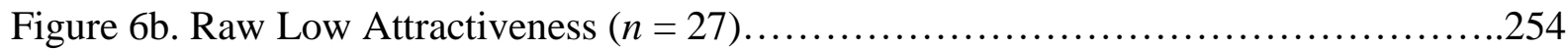

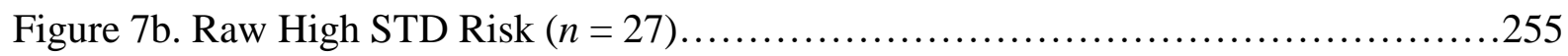

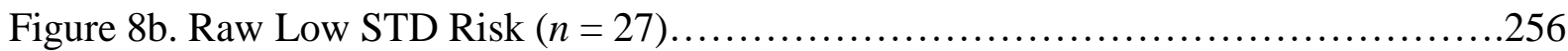

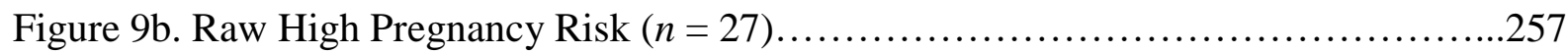

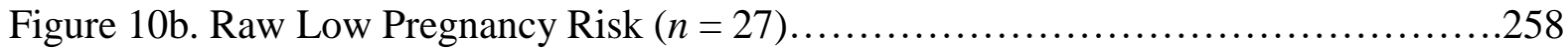

Figure 11b. Incomplete and Complete Data (Monetary) $(n=27) \ldots \ldots \ldots \ldots \ldots \ldots \ldots \ldots \ldots \ldots \ldots . . . \ldots 259$

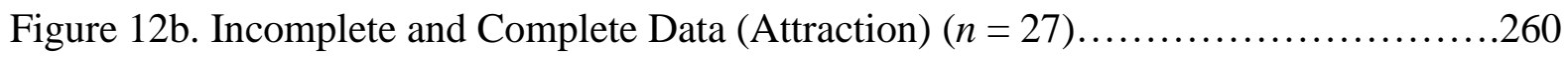

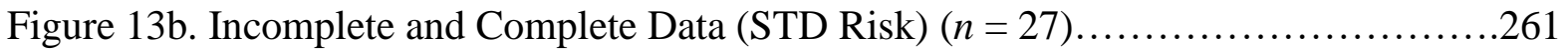


Figure 14b. Incomplete and Complete Data (Pregnancy Risk) $(n=27) \ldots \ldots \ldots \ldots \ldots \ldots . . .262$ 


\section{Differences in Sexual Delay Discounting Among In-Treatment Adults with Opioid Use}

\section{Disorder}

\section{Introduction}

Impulsive decision-making has been observed across various contexts (Dalley, Everitt, \& Robbins, 2011; Rogers, Moeller, Swann, \& Clark, 2010) and is a hallmark of addiction (Moody et al., 2016; Verdejo-Garcia, Lawrence, \& Clark, 2008). For adults with an addiction, impulsive decision making is related to earlier substance use initiation (Dougherty et al., 2015; Verges, Littlefield, Arriaza, \& Alvarado, 2019) and increased risk of relapse among those in treatment (Adinoff et al., 2007; Pattij \& De Vries, 2013). The most pressing addiction concern in the United States of America is opioid use disorder. The country is experiencing an opioid epidemic that has been characterized by record numbers of overdose deaths (Hedegaard, Bastian, Trinidad, Spencer, \& Warner, 2018), high levels of prescription opioid misuse (Han, Compton, Blanco, \&

Jones, 2018), and increasing instances of neonatal abstinence syndrome (Patrick et al., 2012).

Thus, a better understanding of impulsive decision-making in the context of the opioid epidemic is an important public health concern. Using the experimental task of delay discounting, the present study examines how adults with opioid use disorder indicate choices about sexual behaviors.

\section{Delay Discounting}

Delay discounting is both a framework and a task used to understand impulsive decisionmaking (Reynolds \& Schiffbauer, 2005). Impulsivity is a multifaceted construct and delay discounting is one way to characterize impulsivity (Argyriou, Um, Carron, \& Cyders, 2018). The experimental delay-discounting tasks are distinct from action-based and self-report measures of impulsive decision-making (Broos et al., 2012; Xu, Korczykowski, Zhu, \& Rao, 2013). That is, 
delay-discounting tasks have discrete alternatives based on delays while action-based and selfreport measures can rely on subjective interpretation of time and an accurate understanding of your impulsive characteristics in relation to others while delay-discounting tasks have clear delays and do not rely on referring to others to determine your response (Odum, 2011b). As a framework, delay discounting is a way to understand impulsive decision-making when the decisions are both: 1 . temporally relevant (i.e., reward now or later) and 2. the choice is based on subjective evaluation of the reward value rather than objective reward value (Odum, 2011b). Ultimately, delay-discounting tasks provide a way to measure in which domains impulsive, smaller-soon decisions are made (Paglieri, 2013). Due to its wide applicability to a range of decision-making domains that have implications across the life-span (Argyriou et al., 2018), delay-discounting tasks have grown to be an important method to characterize decision-making (Bickel \& Mueller, 2009).

To evaluate the degree to which delay discounting influences an individual's decisionmaking, researchers can use various domain-specific delay-discounting tasks (Green \& Myerson, 2004). Due to the ubiquity and importance of monetary decision-making, delay-discounting tasks have frequently used monetary decisions as a means to examine impulsive choice. In a typical monetary delay-discounting task, the participant makes a series of dichotomous choices between a self-selected smaller-sooner hypothetical monetary reward, such as receiving $\$ 50$ now, as opposed to a receiving a larger-later reward, such as $\$ 100$ in six months (Rachlin, Raineri, \& Cross, 1991). In order to isolate the some of the effects that delay has on choice, the larger-later reward systematically shifts the delay length while maintaining the larger magnitude (e.g., \$100 in one day, $\$ 100$ in one week, $\$ 100$ in one month, $\$ 100$ in six months, $\$ 100$ in one year, $\$ 100$ in five years). At some delay point, a participant will shift their choice from the larger-later reward 
to the smaller-sooner. This shift indicates at what value or delay the subjective value of the smaller-sooner and larger-later are equivalent.

Based on an individual's response at each delay point, there are various ways to compute and compare an individual's preference for smaller-sooner rewards such as $k$, ED50, and area under the curve (AUC; Yoon \& Higgins, 2008). $k$ estimates the fit of the data to a hyperbolic curve (Mazur, 1987). ED50 was proposed to help clarify and standardized the reporting of delaydiscounting task results in the medical literature by using an already recognized model, the effective delay 50 (i.e., ED50, Yoon \& Higgins, 2008). Both $k$ and ED50 rely on participants choosing a smaller-sooner reward that is $50 \%$ of the delayed magnitude at some point during the task (Yoon \& Higgins, 2008). In delay-discounting tasks with shorter delays, this amount of discounting may not occur. AUC provides an assumption-free outcome variable derived from a participant's discounting data (Myerson, Green, \& Warusawitharana, 2001). That is, unlike with $k$, there are no underlying assumptions that the data fits a hyperbolic curve (Yoon et al., 2017). Fit for AUC uses a participant's responses at each delay to compute AUC, while $k$ fits a participant's responses to the hyperbolic function (Odum, 2011a). These limitations of $k$ and ED50 are especially pronounced when using delay-discounting tasks that utilize shorter time delays (i.e., minutes and hours) because they often lack hyperbolic discounting, thus AUC is the preferred output for (Johnson et al., 2017; Stoltman et al., 2015). There are limitations to AUC when steep discounting occurs (i.e., more preference for smaller sooner) and in those situations, $k$ or ED50 may be preferred approaches (Yoon et al., 2017). Overall, a choice for smaller-sooner rewards is considered to be an impulsive choice and AUC is one way that this choice can be represented (Green, Myerson, Oliveira, \& Chang, 2013). 
Sexual Discounting Task. Although sexual discounting tasks have grown in popularity over the past decade (Berry \& Johnson, 2018), there has been relatively little investigation into individual differences that might underlie sexual decision-making processes using this task. Sexual decision-making is an emotionally salient process and can shift based on delays, making it a prime candidate for understanding using a sexual discounting task (Lawyer, Williams, Prihodova, Rollins, \& Lester, 2010). Initial research in sexual discounting focused on task development (Johnson \& Bruner, 2012) and populations with substance use disorders (for review, see: Berry \& Johnson, 2018). These initial studies using the Sexual Discounting Task often used the same delay lengths as monetary tasks to determine the domain level differences between sexual decision-making and monetary decision-making. A new, adapted version of the sexual discounting task has since been developed that uses ecologically relevant time-frames (Johnson, Herrmann, Sweeney, LeComte, \& Johnson, 2017). Inquiry into sexual decisionmaking with these ecologically relevant time frames (i.e., minutes rather than weeks and years) needs further investigation to understand whether these shifts can happen across ecologically relevant delay lengths. Additional research related to partner characteristics (i.e., attractiveness, STD risk, pregnancy risk) is needed. Such information may advance our understanding of the circumstances in which individuals discount, enabling more tailored interventions and sexual health psychoeducation programs.

\section{Domains, Delay Length, Reward Magnitude, and Individual Differences}

One benefit of using delay-discounting tasks to assess decision-making is the flexibility to change the rewards used in the task based on the domain of interest (e.g., food choice or condom use). In some instances, the delay discounting has been shown to be affected by domain (Field, Santarcangelo, Sumnall, Goudie, \& Cole, 2006; Giordano et al., 2002; Mitchell, 2004; 
Stoltman, Woodcock, Lister, Lundahl, \& Greenwald, 2015). Thus, because domain can influence impulsive choice, domain-specific decision-making investigations should use domain-specific rewards and delays. The development of the Sexual Discounting Task is one attempt to better understand these domain-specific decision-making processes by using a domain-specific reward (e.g., hypothetical sexual intercourse). Aside from reward type, when choosing to develop a new task or use an existing one, a researcher must also consider delay length and reward magnitude (Weatherly, 2014).

The delay length chosen for a discounting task should be based on the relevant temporal structure of the decision-making process of interest. For example, the delay lengths initially preferred for monetary tasks (i.e., weeks, months, years) were selected to approximate delays encountered in real-world long-term financial decisions (e.g., using a savings account or 401k) and explain under what conditions people generally favor smaller-sooner versus larger-later hypothetical monetary rewards (Kirby \& Marakovic, 1996). As such, monetary delaydiscounting tasks typically have delay lengths that ranged from weeks to months to years (Odum \& Rainaud, 2003). However, to accurately represent domains that have decisions with a more immediate hedonic value, such as sexual decision-making or substance use, delay lengths on the order of minutes and hours may have better face validity and ecological validity (Johnson et al., 2017; Stoltman et al., 2015). While many decisions have both short and long-term implications, sexual activity with an immediately available and willing sexual partner would likely not be delayed for months or years. A second consideration when choosing delay lengths is how these delays fit into the larger delay-discounting literature. To appropriately compare discounting tasks and better understand the domain-related factors that can affect the preference for smaller-sooner rewards, it is important to use a similar delay length so that the two tasks are modeling similar 
decision-making parameters. That is, by using the same delays regardless of domain, a researcher can isolate differences in cross-over point between two domains as a product of the reward type, not the delay length. In summary, when choosing delay length for the delay-discounting task, domain matters; thus, reasonable time delays should be used when possible to increase ecological validity.

Reward magnitude is another important consideration. Although reward magnitude (e.g., $\$ 100$ or $\$ 1000$ ) has not been consistently shown to effect the choice for smaller-sooner rewards across the life-span in monetary delay-discounting tasks (Green, Myerson, Oliveira, \& Chang, 2014; Odum, Baumann, \& Rimington, 2006), this might be specific to the domain of monetary decision-making. That is, while monetary magnitude is objective, sexual "magnitude" may be more subjective in nature and thus, more susceptible to differences in impulsive choice across conditions. Previous attempts to quantify sexual magnitude have elucidated differences between high/low attractiveness (Johnson \& Bruner, 2012); however, the procedures have been cumbersome. In this task, a participant rated 60 pictures based on attractiveness and sorted them into high/low attractiveness (Johnson \& Bruner, 2012). Thus, development of streamlined research protocol that allows for testing the role magnitude might have on domain-specific decision-making, would be beneficial to the field.

Life-span specific considerations. Because research has focused on task parameters, less attention has been paid towards potential individual differences in the sexual-discounting literature. Life-span developmental psychology is interested in individual differences in growth and decline across the life-span (Baltes, 1987). Sexual decision-making is a complex process that can be affected by various contextual factors (Carrier Emond, Nolet, Cyr, Rouleau, \& Gagnon, 2016) and individual differences such as: future time perspective (Sosa-Rubí, Salinas-Rodríguez, 
Montoya-Rodríguez, \& Galárraga, 2018); sexual arousal (Ariely \& Loewenstein, 2006;

Jarmolowicz et al., 2014; Johnson et al., 2017; Lawyer \& Schoepflin, 2013); condom attitudes (Shih et al., 2011); and gender (Johnson \& Bruner, 2013; Lawyer \& Schoepflin, 2013). As highlighted in this section, these individual differences have been addressed to a limited degree in sexual-discounting literature. Due to the importance of understanding risky sexual decisionmaking, better understanding these person-level processes can be an important step in developing efficacious interventions to reduce risky sexual decision-making.

Gender differences in sexual decision-making have not been studied extensively using sexual discounting tasks. The limited research in the area of sexual discounting suggests women prefer waiting to use a condom more than men (Johnson \& Bruner, 2013; Lawyer \& Schoepflin, 2013). Although, this conclusion is derived from the original Sexual Discounting Task which used longer delays (i.e., months and years) and smaller samples ( $n=13$ and $n=66$ women, respectively), which could affect the interpretation and generalizability of these findings. These initial inquiries suggest additional inquiry is needed to increase our understanding of this gender differences.

The nascent research in sexual discounting has had limited overlap with time perspective research, although there is a reason to believe that differences in time perspective can affect general decision-making processes. Future time perspective is defined as the extent to which individuals view their future as distinct from their past and present (Brothers, Chui, \& Diehl, 2014). Socioemotional Selectivity Theory theorizes that this perception of time left, as opposed to time passage or duration, is associated with decision-making (Carstensen, Isaacowitz, \& Charles, 1999). Socioemotional Selectivity Theory provides a decision-making framework that postulates decision-making is associated with valuations of time (open or restricted) and that the 
perception of time as finite, or close to the end, changes our evaluation of goals and behaviors (Carstensen, 1991). In a study of male sex workers in Mexico, those with higher future time perspective had reported higher condom use (Sosa-Rubí et al., 2018). Additionally, future time perspective was not associated with condom use with casual partners, but greater orientation toward the future was associated with more frequent condom use with regular partners (SosaRubí et al., 2018). The seemingly inconsistent association of future time perspective with condom use could be due to the contingencies surrounding condom-less sexual intercourse for sex workers, that is with casual partners condom use were overall lower but financial incentives could be driving this behavior more than time perspective (Sosa-Rubí et al., 2018). Other research has shown that future time perspective is related to monetary delay-discounting (Cosenza, Griffiths, Nigro, \& Ciccarelli, 2017; Gollner, Ballhausen, Kliegel, \& Forstmeier, 2017); however, monetary delay-discounting has also been shown to be a distinct type of decision-making when compared to sexual discounting (Jarmolowicz, Bickel, \& Gatchalian, 2013; Johnson \& Bruner, 2012). Taken together, further research is needed into the role future time perspective might have on these sexual decision-making processes because time perspective is malleable and thus could inform future intervention development if it is a relevant variable (Stein, Tegge, Turner, \& Bickel, 2018; Stein et al., 2016).

Sexual intercourse-specific variables. Sexual arousal has been shown to drive more impulsive sexual decision-making both in real-world (Ariely \& Loewenstein, 2006) and hypothetical situations (Jarmolowicz et al., 2014; Lawyer \& Schoepflin, 2013), but not overall discounting (Lawyer \& Schoepflin, 2013). Increases in sexual arousal have been shown to be associated with riskier sexual decision-making, and increased risk of exposure to sexually 
transmitted disease (Johnson et al., 2017). Taken together, sexual arousal is an important variable to consider whether it might affect sexual decision-making surrounding pregnancy risk.

Condom attitudes are thought to influence condom use (Shih et al., 2011). When evaluating condom use, there can be differences in affective and cognitive components for women (Hood \& Shook, 2013), though this has not been fully assessed in men. For women, having a positive attitude toward condoms was positively associated with past condom use, while having positive thoughts about condoms was associated with future condom use intentions (Hood \& Shook, 2013). Condom use intentions, however, are not always realized in heat of the moment decision-making scenarios like sexual activity as shown above by the work of Ariely and Loewenstein (2006). More work is needed to disentangle the role condom attitudes might have on hypothetical condom use in risky situations and whether this is a factor for men.

Higher rates of risky sexual behavior can be observed in adults with substance use disorder, making this sample an important at-risk group to understand (Woerner, Kopetz, Lechner, \& Lejuez, 2016). However, relatively little else is known about what might predict sexual discounting, whether these domain level decision-making factors differ at shorter delays, or whether ecologically valid tasks (i.e., with shorter delays) might increase our understanding of these processes.

\section{The Opioid Epidemic}

The United States is experiencing a wide-spread opioid epidemic (Murthy, 2016). The present opioid epidemic is characterized by direct effects such as the high rates of opioid use/misuse (Han et al., 2018) and overdose deaths (Hedegaard et al., 2018), as well as indirect effects such as increased rates of sexually transmitted infections (STI) and sexually transmitted disease (STD) e.g., Hepatitis C virus and HIV transmission (Linley et al., 2018; Zibbell et al., 
2018). HIV and Hepatitis C virus transmission have a multifaceted relation with the opioid epidemic due to the dual risk of transmission associated with injection drug use and risky sex (Shaw \& Hunter, 2012; Tibbs, 1995), making sexual risk behaviors a particularly important area of study. As such, the limited research on sexual discounting in opioid-using individuals has focused on understanding under which conditions people are likely to engage in risky sex that might lead to STD transmission. The interaction between risky sexual behaviors and drug use are critically important to understand and inform intervention development, but they are not the only risks associated with condom-less sex.

Another area of concern is the disproportionate effect of risky sex on women with opioid use disorder. The sequelae of risky sex are unbalanced between the genders because women are also at risk for unintended pregnancy. Women already experience more complications with opioid use disorder, as seen through the higher number of symptoms when presenting for opioid use disorder treatment and higher rates of relapse (Mack et al., 2017). Because of these disproportionate effects, research about sexual-health behaviors in opioid use disorder has primarily focused on women; however, sexual behavior and sexual decision-making often involves both men and women, suggesting that our understanding of sexual health behavior in opioid use disorder can benefit from an extended scope to include both genders and include questions related to pregnancy risk. Overall, substance use disorder intersects with human sexuality in complex ways.

\section{Limitations of Prior Research}

Although sexual delay discounting is still a new area of delay discounting, methodological advancements can widen the reach and applicability of the Sexual Discounting Task. With the high prevalence of unintended pregnancies (Heil et al., 2011) and limited use of 
high efficacy birth control in substance use disorder patient populations (Terplan, Hand, Hutchinson, Salisbury-Afshar, \& Heil, 2015), sexual discounting research can inform our understanding of circumstances surrounding unintended pregnancies and STD transmission. To date, much of the general research in sexual health and opioid use disorder has focused on unintended pregnancy rate and understanding birth control use. In research on sexual delay discounting, the focus has been on STIs/STDs and partner attractiveness as reasons that might shift condom use. Extending sexual discounting research to include attractiveness, STD risk, and pregnancy risk can help inform the development of precise interventions. This study is among the first to develop a pregnancy-risk delay discounting task condition and propose that a participant's evaluation of pregnancy-risk might shift preference away from waiting until a delayed condom is available.

The majority of earlier sexual delay discounting work used longer delays (e.g., weeks, months) to compare with monetary delay-discounting tasks. Few investigators have included individual difference characteristics such as gender, time perspective, sexual arousal, or condom attitudes to understand how these might affect hypothetical condom use. This study will characterize condom use decisions across various conditions using a delay-discounting framework and to incorporate individual differences to further our understanding of condom decision-making in adults with opioid use disorder. Due to the opioid epidemic, there is a pressing need to understand more about this sub-group through the developmental focus of individual differences.

\section{Problem Statement}

Adults with substance use disorders often exhibit more impulsive decision-making (Moody et al., 2016; Verdejo-Garcia et al., 2008). Sexual decision-making is complex and can 
lend itself to increased impulsive decision-making, which can have long-term consequences (Ariely \& Loewenstein, 2006). Understanding factors that influence sexual decision-making can help support the development of high efficacy interventions in adults with substance use disorder who may be at higher risk for sequelae associated with risky sexual decision-making.

\section{Research Questions and Hypotheses}

This study was guided by several research questions and hypotheses, as detailed below:

RQ1. Are there domain differences in delay discounting?

H1a. Prior research has not directly assessed differences between monetary and sexual discounting; however, non-significant correlations in previous research indicate differences could be present (Johnson \& Bruner, 2012, 2013; Johnson, Johnson, Herrmann, \& Sweeney, 2015). I hypothesized that sexual discounting would have lower AUC (i.e., more preference for smaller sooner rewards) than monetary discounting.

RQ2. Does reward magnitude influence choice for monetary and sexual discounting?

H2a. Based on previous research in monetary discounting (Green, Myerson, Oliveira, \& Chang, 2013), I hypothesized that there would be differences in AUC within monetary delay discounting between the two conditions, $\$ 10$ versus $\$ 100$. I hypothesized that the $\$ 10$ magnitude would have lower AUC (i.e., more preference for smaller sooner rewards) than the $\$ 100$ magnitude.

H2b. Based on previous research in sexual discounting (Johnson et al., 2017), I hypothesized that there would be differences between sexual discounting based on attractiveness. I hypothesized that the High Attraction condition would have lower AUC (i.e., more preference for immediate, condom-less sexual intercourse) compared to the Low Attraction condition. 
H2c. Based on previous research in sexual discounting (Herrmann, Hand, Johnson, Badger, \& Heil, 2014; Johnson \& Bruner, 2012; Johnson et al., 2017; M. W. Johnson et al., 2015), I hypothesized that there would be differences between sexual discounting based on STD risk. I hypothesized that the Low STD Risk condition would have lower AUC (i.e., more preference for immediate, condom-less sexual intercourse) than the High STD Risk condition.

H2d. The pregnancy risk task is new but based on previous studies investigation into STD risk and sexual discounting, I hypothesized there would be differences between sexual discounting conditions based on riskiness. I hypothesized that the Low Pregnancy Risk condition would have a lower AUC (i.e., more preference for immediate, condom-less sexual intercourse) than the High Pregnancy Risk condition.

RQ3. Are differences in sexual discounting associated with individual differences?

H3b. The inclusion of subscales that measure Future Time Perspective is novel in the sexual discounting literature. Based on previous research in Future Time Perspective (Cosenza et al., 2017; Gollner et al., 2017; Sosa-Rubí et al., 2018) and SST (Carstensen, 1991), I hypothesized that Future Time Perspective would be correlated with the sexual discounting AUC. Higher endorsement of Future as Open would be associated with lower AUC (i.e., more preference for immediate, condom-less sexual intercourse) in the High Attraction, Low STD Risk, and Low Pregnancy Risk conditions. Higher endorsement of Future as Limited would be associated with higher AUC (i.e., less preference for immediate, condom-less sexual intercourse) in the High Attraction, Low STD Risk, and Low Pregnancy Risk conditions. Higher endorsement with Future as Ambiguous would be associated with lower AUC (i.e., more preference for immediate, condom-less sexual intercourse) in the High Attraction, Low STD Risk, and Low Pregnancy Risk conditions. 
H3c. Based on previous research in sexual discounting and decision-making (Ariely \& Loewenstein, 2006; Jarmolowicz et al., 2014; Johnson et al., 2017), I hypothesized that sexual arousal would be negatively correlated with the sexual discounting AUC for all six sexual discounting conditions. Higher sexual arousal would be associated with lower AUC (i.e., more preference for immediate, condom-less sexual intercourse).

H3d. The inclusion of subscales that measure condom attitudes is novel in the sexual discounting literature. Based on previous research in sexual decision making (Hood \& Shook, 2013; Shih et al., 2011), I hypothesized that condom attitudes would be correlated with the sexual discounting AUC for all six sexual discounting conditions. Higher endorsement with MCAS Condom Pleasure would be associated with higher AUC (i.e., less preference for immediate, condom-less sexual intercourse) in all six sexual discounting conditions. Higher endorsement with MCAS Identity Stigma Related to Condom Use would be associated with higher AUC (i.e., less preference for immediate, condom-less sexual intercourse) in all six sexual discounting conditions. Higher endorsement with MCAS Embarrassment About Negotiation and Use of Condoms would be associated with higher AUC (i.e., less preference for immediate, condom-less sexual intercourse) in all six sexual discounting conditions.

H3a. Based on previous research in sexual discounting (Jarmolowicz et al., 2014; Johnson \& Bruner, 2013; Lawyer \& Schoepflin, 2013), I hypothesized that there would be differences in sexual discounting AUC based on gender. I hypothesized that men would have a lower AUC (i.e., more preference for immediate, condom-less sexual intercourse) than women across all six sexual discounting conditions.

\section{Method}

\section{Study Design}


Participants were drawn from a brief-longitudinal study on sexual health and opioid treatment (for more information on the full list of measures included in the study, see Appendix A). In total, 128 participants were enrolled in the study. The typical participant was Caucasian (92.2\%) women $(72.2 \%)$ nearly 34 years of age $(M=33.8, \mathrm{SD}=8.5$ years; range: $19-69$ years $)$ and $88.5 \%$ had a high school education or higher $(M=12.6, \mathrm{SD}=1.9$ years; range: $6-17$ years $)$. Recruitment occurred from 2016 to 2018 at the Comprehensive Opioid Addiction Treatment (COAT) Clinic at West Virginia University. The COAT Clinic is a combination sublingual naloxone/buprenorphine medication-assisted treatment program serving patients with primary opioid use disorder diagnoses (Zheng et al., 2017). All participants included in this analysis provided self-report information using the 7" Amazon Fire capacitive touch tablet to answer questions using REDCAP (Obeid et al., 2013) and Qualtrics (Provo, UT) survey software. All the survey collection devices used stock Amazon Fire Tablet OS software and Chrome web browser to administer the online surveys. To be eligible, potential participants had to be 18 or older, have an opioid use disorder diagnosis, and be actively enrolled in the opioid use disorder treatment. The West Virginia University Clinical and Translational Science Institute and Behavioral Medicine and Psychiatry at West Virginia University provided study funding. The West Virginia University Institution Review Board approved this study. For additional procedural information, refer to Appendix B.

Survey items were based on relevant questions from national surveys and review of the sexual health literature. The larger study was designed to track the change in sexual health behavior over time, which necessitated multiple survey waves. Wave 1 focused on sexual health, contraceptive knowledge, and access to sexual health services. Wave 2 focused on monetary and sexual discounting. Wave 3 followed up many of the questions from Wave 1 and included 
additional questions on drug use progression, coercive sex, and intimate partner violence. See Appendix A for more information on the full list of measures.

\section{Procedure}

Study recruitment consisted of both word-of-mouth and a brief in-person description of the study to a treatment group (maximum size of 12 people) by trained research staff. Participants were recruited immediately before the start of that day's group-based medicationassisted treatment appointment. After that day's treatment group ended, adults interested in taking part in the study were provided further details about the study and consented. Research staff answered questions about the study during this time. Those interested in participating were then group consented. Consent processes included a brief description of the study, information on financial considerations, HIPAA, and the voluntary nature of research participation (Appendix A). Informed consent was provided by 128 adults prior to study participation. All participants completed the consent process and survey in a group meeting room that provided a quiet, confidential environment. Honoraria was provided as a \$10 Walmart gift card for Wave 1, \$15 Walmart gift card for Wave 2, and \$25 Walmart gift card for Wave 3 totaling three gift cards if all study waves were completed.

After consent, all participants were provided a confidential ID number to link the multiple survey waves. Tablet computers were then provided to each participant, with time spent adjusting the font and orienting each participant to the touch-screen device. For Wave 2, participants were shown how to rotate the tablets for the delay discounting tasks. The participants self-advanced the survey software. Participants who needed a rubber-tipped nonactive stylus (e.g., long fingernails) were provided one. Research staff remained in the room to answer questions and troubleshoot device issues. 
Survey Wave 2 (Delay Discounting) was broken up into six parts. Part 1 began with a brief series of questions on demographic information. The participants then received on-screen instructions to rotate the tablet computer from vertical to horizontal. Part 2 consisted of a Dinner Practice session (Johnson et al., 2017) to orient the participant to the task and troubleshoot any task administration issues (e.g., non-responsive touch-screen). Part 3 included the Monetary Delay-Discounting Task (Johnson, Herrmann, \& Johnson, 2015) with a block randomizer. Part 4 included the Sexual Discounting Task (Johnson et al., 2017) with a block randomizer that encompassed the high/low attractiveness and STD high/low-risk conditions. Part 5 included the novel Pregnancy Risk Sexual Discounting Task with a block randomizer for high/low-risk. Prior to Part 6, the participants received on-screen instructions to rotate the tablet computer from horizontal to vertical before completing included sex-specific questions on sexual history. There were no breaks between survey parts, and the overall session took approximately 15 minutes. More detail on each relevant part of the survey will be provided below.

Survey software. Study data for Wave 1 and Wave 3 were collected and managed using REDCap electronic data capture tools hosted at West Virginia University. Wave 2 data were collected using Qualtrics (Provo, UT) to facilitate completion of the discounting tasks, which used a visual analog scale (VAS). Prior to initiating the delay discounting portion of the survey on the tablet, participants were instructed in the survey and by research staff to rotate the survey horizontally to allow the largest surface area possible for completing the delay discounting tasks with the VAS pointer. The VAS was 5.5 inches long when completed horizontally. The tablet registers multiple points of contact and has a pixel density of 171 pixels per inch. Participants were provided rubber tipped non-active styluses for completing the delay discounting tasks with the VAS pointer if they were having difficulty with the tablet registering manual touch input. 
The rubber tipped non-active stylus only registers an input when placed on the screen. When moving the VAS pointer, the number reflecting the current position of the VAS pointer automatically populated next to the line. For all discounting tasks described herein, the default VAS pointer location started at $50 \%$ and only after the VAS pointer moved did the current VAS pointer location populate next to the line. To move to the next item, the VAS pointer had to move from its default position. For additional procedural information, refer to Appendix B.

\section{Measures}

Gender. Self-reported as either man or woman in the demographics of each study wave. Future Time Perspective. At the end of Wave 1, all participants were asked questions on time perspective. The present study used a recently developed brief, 12-item measure of future time perspective (Brothers et al., 2014). The final 12-item measure has three distinct components: Future as Open, Future as Limited, and Future as Ambiguous (Brothers et al., 2014). Items were endorsed on a 5-point Likert-type scale from "Strongly Disagree" to "Strongly Agree." Exemplar items include "My future is uncertain" and "I know that I do not have all the time in the world." Reliability was acceptable to good for all 3-subscales included in the present analysis: Future as Open $(\alpha=0.79)$, Future as Limited $(\alpha=0.76)$, and Future as Ambiguous $(\alpha=$ 0.84). The full list of items in in Appendix A.

Sexual Arousal. Before each condition, participants were prompted to think of themselves as single and available to have sex. Next, participants were asked to rate "How interested are you in having sex right now?" from 0-100 using a VAS pointer. Both poles were labeled as Not Interested (0) and Very Interested (100). The percent represented by the VAS pointer position was populated directly next to the VAS. The default VAS pointer position was $50 \%$. To move to the next item, the VAS pointer had to move from its default position. 
Condom Attitudes. At the end of Wave 1, participants were presented questions about condom attitudes using the UCLA Multidimensional Condom Attitudes Scale (MCAS; HelwegLarsen \& Collins, 1994). To reduce participant burden, 3-subscales (Condom Pleasure, Identity Stigma Related to Condom Use, Embarrassment About Negotiation and Use of Condoms) totaling 14-items were used from the larger 5-subscale, 25-item questionnaire (Helweg-Larsen \& Collins, 1994). These 3-subscales were chosen for their relevance to this sub-group. Items were endorsed on a 7-point Likert-type scale from "Strongly Disagree" to "Strongly Agree" (HelwegLarsen \& Collins, 1994). Exemplar items include "Use of a condom is an interruption of foreplay" and "It is easy to suggest my partner use a condom." Reliability was acceptable to good for the 3-subscales used in the present analysis: MCAS Condom Pleasure $(\alpha=0.69)$, MCAS Identity Stigma Related to Condom Use $(\alpha=0.88)$, and MCAS Embarrassment About Negotiation and Use of Condoms $(\alpha=0.81)$. The full list of items in in Appendix A.

Dinner Task: Practice. After demographics questions in Wave 2, a practice session was used to acclimate participants to the question format, identify any questions participants had about the task, identify problems with the using the VAS on the tablet, and emphasize reading the prompt for each portion of the task. Previous studies have also used a simulated practice session to allow for questions and determine ability to complete the task (Johnson et al., 2017). More information on the practice task is provided in Appendix A and Appendix B.

Monetary Delay-Discounting Task. The present study used an adapted version of the hypothetical \$10 and \$100 magnitude monetary rewards conditions (P. S. Johnson et al., 2015). The "free condition" was chosen because it reduces the role opportunity costs might have on monetary delay discounting (P. S. Johnson et al., 2015). In this task, opportunity costs refer to the ability to gain money through other sources during the wait for the larger-later delayed 
reward (Paglieri, 2013). The full Monetary Delay-Discounting Task prompt is provided in Appendix A. Data derived from the two Monetary Delay-Discounting Task magnitudes was used to compute AUC as described in the data analysis section below.

The eight delay lengths were presented in order and ranged from minutes to hours (5 min.; 10 min.; 30 min.; 1 hr.; 3 hr.; 6 hr.; 12 hr.; 24 hr.). Participants used a VAS pointer to reflect how much money now would feel just as good as the larger-later amount (either \$10 or $\$ 100$ based on the condition). For example, in the $\$ 100$ magnitude condition and delay of 6 hours, a participant could move their VAS pointer to $\$ 75$ now to reflect that $\$ 75$ now would feel just as good as $\$ 100$ in 6 hours. Moving the VAS pointer to $\$ 100$ would reflect that the largerlater delayed amount was preferred. Similar to the practice session, the dollar amount represented by the VAS pointer position was populated directly next to the VAS. The default VAS pointer position was either $\$ 5$ and $\$ 50$ respectively for each magnitude and each delay length. To move to the next item, the VAS pointer had to move from its default position. The condition order was randomized using the block randomizer in Qualtrics (i.e., \$10 condition than $\$ 100$ condition or $\$ 100$ condition than $\$ 10$ condition).

Sexual Discounting Task. The present study used hypothetical desire and STD risk conditions adapted from the Sexual Discounting Task (Johnson \& Bruner, 2012; Johnson et al., 2017). Test-retest reliability of this task has shown good stability over a one-week timeframe (Johnson \& Bruner, 2013). An additional pregnancy risk condition was developed specifically for this project. In total, six sexual delay discounting conditions were used: high attractiveness, low attractiveness, high STD risk, low STD risk, high pregnancy risk, low pregnancy risk.

Before each condition, participants were prompted to think of themselves as single and available to have sex. Next participants were asked to rate their attractiveness and desire to have 
sex right now from 0-100 using a VAS. For attractiveness, the poles were Very Unattractive (0) and Very Attractive (100). For desire, the poles were Not Interested (0) and Very Interested (100). An adapted version of the classic Johnson and Bruner (2012) Sexual Discounting Task was used whereby the participant was asked to imagine an individual rather than complete the picture rating process. This is the first study to rely on hypothetical sexual partners instead of the picture rating process. Each condition had specific instructions for the participant to imagine a hypothetical person. For illustration purposes, in the MOST attractive condition, participants were asked to "imagine the person you would MOST want to have sex with; you are very interested in have sex with this individual" and then rate that individual using a similar VAS to the self-attractiveness rating, 0-100 with both poles were labeled Very Unattractive (0) and Very Attractive (100). The participant was then prompted:

Imagine the person you would $\underline{\text { MOST }}$ want to have sex with. You are very interested in having sex with this individual. Imagine that you have just met this person. You are getting along great and they are interested in having sex with you now. Imagine you are confident that there is no chance of pregnancy, for example, you know that one of you is either on the pill, has had their "tubes tied," or had a vasectomy. Imagine that there is a condom readily and immediately available.

The eight delay lengths were presented in order and ranged from an immediately available condom to delayed ability to use a condom from minutes to hours (immediately available; 2 min.; 5 min.; 15 min.; 30 min.; 1 hr.; 3 hr.; 6 hr.). Participants used a VAS pointer to reflect how likely they were to wait to use a condom across the conditions at different delay lengths. Each pole was labeled as "I will definitely have sex with this person right away without a condom (0)" and "I will definitely wait DELAY LENGTH to have sex with this person with a condom 
(100)." For example, in the MOST attractive condition and delay of 6 hrs., a participant could move the VAS pointer to $30 \%$ to reflect that they are $30 \%$ likely to wait 6 hrs. to use a condom when having sex with this person. Similar to the practice session, the percent represented by the VAS pointer position was populated directly next to the VAS. The default VAS pointer position was $50 \%$ for each condition. To move to the next item, the VAS pointer had to move from its default position.

The condition order was randomized using the block randomizer in Qualtrics. To remain consistent with previous research (Johnson et al., 2017), multiple blocks were used for randomization procedures. Block 1 randomized between and within attractiveness and STD conditions. Block 2 used the novel Pregnancy Risk Task and was thus randomized separately. Block 2 randomized the order of pregnancy risk conditions. For more detail, refer to Appendix A and $\mathrm{C}$ for the randomization of the Sexual Discounting Task.

Due to the length of this survey and potential repetitiveness of using a VAS for a significant portion of the survey (i.e., two Monetary Delay-Discounting Tasks and six Sexual Discounting Tasks), quality checks were inserted after the first two conditions and in the middle of the pregnancy risk condition. The quality check consisted of typing the words "West Virginia" and moving the VAS pointer to $75 \%$ before the participant was able to advance to the next task. The full Sexual Discounting Task prompt is provided in Appendix A. Data derived from the six Sexual Discounting Task conditions will be used to compute AUC as described in the data analysis section below.

Sexual Discounting Task: Attractiveness. There were two attractiveness conditions that participants were asked to imagine: most and least (Johnson \& Bruner, 2012). For the most attractive condition, participants were asked to "imagine the person you would most want to 
have sex with; you are very interested in have sex with this individual" and then rate that individual using a similar slide to the self-attractiveness rating, 0-100 with both poles were labeled Very Unattractive (0) and Very Attractive (100). The participant was then prompted:

Imagine the person you would MOST want to have sex with. You are very interested in having sex with this individual. Imagine that you have just met this person. You are getting along great and they are interested in having sex with you now. Imagine you are confident that there is no chance of pregnancy, for example, you know that one of you is either on the pill, has had their "tubes tied," or had a vasectomy. Imagine that there is a condom readily and immediately available.

Participants then proceeded to rate the likelihood that they would wait to use a condom with progressively longer presented in order and ranged from immediately available condom to delayed from minutes to hours (immediately available; 2 min.; 5 min.; 15 min.; 30 min.; 1 hr.; 3 hr.; $6 \mathrm{hr}$.). The same procedure was followed for the least attractive condition.

Sexual Discounting Task: STD risk. There were two STD risk conditions that participants were asked to imagine: high and low (Johnson \& Bruner, 2012). For the high STD risk condition, participants were asked to "imagine the person you believe is most likely to have a sexually transmitted disease (STD)" and then rate that individual using a similar slide to the self-attractiveness rating, 0-100 with both poles were labeled Very Unattractive (0) and Very Attractive (100). The participant was then prompted:

Imagine a person you believe is MOST likely to have a sexually transmitted disease (STD). Imagine that you have just met this person. You are getting along great and they are interested in having sex with you now. Imagine you are confident that there is no chance of pregnancy, for example you know that one of you is either on the pill, has had 
their "tubes tied," or had a vasectomy. Imagine that there is a condom readily and immediately available.

Participants then proceeded to rate the likelihood that they would wait to use a condom with progressively longer presented in order and ranged from immediately available condom to delayed from minutes to hours (immediately available; 2 min.; 5 min.; 15 min.; 30 min.; 1 hr.; 3 hr.; $6 \mathrm{hr}$.). The same procedure was followed for the least likely to have an STD condition.

Sexual Discounting Task: Pregnancy risk. The novel Pregnancy Risk Discounting Task was adapted from STD and attractiveness Sexual Discounting Tasks (Johnson \& Bruner, 2012). There were two pregnancy risk conditions that participants were asked to imagine: high and low. For the high pregnancy risk condition, participants were asked to "imagine a person who, if you have sex with them, you are very likely to cause a pregnancy" and then rate that individual using a similar slide to the self-attractiveness rating, 0-100 with both poles were labeled Very Unattractive (0) and Very Attractive (100). The participant was then prompted:

Imagine a person who, if you have sex with them, you are VERY LIKELY to cause a pregnancy. Imagine that you have just met this person. You are getting along great and they are interested in having sex with you now. Imagine you are confident that having sex with this person is $\underline{V E R Y L I K E L Y}$ to cause a pregnancy. Imagine that there is a condom readily and immediately available.

Participants then proceeded to rate the likelihood that they would wait to use a condom with progressively longer presented in order and ranged from immediately available condom to delayed from minutes to hours (immediately available; 2 min.; 5 min.; 15 min.; 30 min.; 1 hr.; 3 hr.; $6 \mathrm{hr}$.). The same procedure was followed for the very UNlikely to cause a pregnancy.

\section{Data Processing}


Basic descriptives were used to characterize the sample, and all variables were assessed for missing data and outliers. Variables violating normality (z scores > 3.29; Field, 2013) were transformed or non-parametric analysis were used if the transformation was not sufficient. Nonnormal distributions (skewness, kurtosis) were transformed using logarithmic or square root transformation depending on the severity of the skewness and need to use normally distributed variables for analysis. Depending on the variables missing and pattern of missingness, participants with missing data were excluded. Spearman bivariate correlations were used to assess the statistical relation between personality, gender, future time perspective, sexual arousal, and condom attitudes. Delay discounting were compared two ways: 1. AUC were used to compare delay discounting between and within domains (e.g., high magnitude monetary versus high STD risk) with a Freidman's Test and a Wilcoxon Signed-rank Tests, 2. "Immediately Available" Condom Preferences, the 0-delay time point, were used as a comparison between and within Sexual Discounting Task conditions.

Delay discounting data cleaning. Discounting data were screened for random or nonsystematic responding using the two criteria set forth by Johnson and Bickel (2008). Criterion 1 was developed through a data driven approach to screen out random responding through assessing if participants responses indicate increased preference for delayed rewards (Johnson \& Bickel, 2008). Criterion 1 violations can suggest random responding or not understanding the task and therefore Criterion 1 violations indicate invalid data that is typically removed case-wise from analysis (Johnson \& Bickel, 2008). For example, in the \$100 monetary task, a participant with increased preference for delayed rewards might select $\$ 5$ now makes me feel just as good as $\$ 100$ at the 2 minute delay. At the 180 minute delay, this same participant would select $\$ 100$ now makes me feel just as good as $\$ 100$ at the 180 minute delay. This suggests that when the 
delay increased from 2 minutes to 180 minutes, the value of delayed rewards increased. That is, when the delay increased, more immediate money was needed to feel as good as the delayed amount. This response pattern indicates that an increased delay increased the value of the delayed reward. This response pattern is counter-intuitive and indicates that the participant might not understand the task or be randomly responding, thus a response pattern like this would be flagged by the algorithm as violating Criterion 1 (Johnson \& Bickel, 2008). To systematically identify Criterion 1 violations, Johnson and Bickel (2008) recommend identifying cases where there is $\mathrm{a} \geq 10 \%, 20 \%$, or $30 \%$ difference between adjacent delays. Due to the use of a touchscreen and use of a research naïve sample, this study used the liberal $\geq 30 \%$ criteria.

In instances where the previous response was under 10\% in the Sexual Discounting Task, under $\$ 1$ in the $\$ 10$ Monetary Delay-Discounting Task, and under $\$ 10$ in the $\$ 100$ Monetary Delay-Discounting Task, the $\geq 30 \%$ criterion will be ignored. For responding under $10 \%$ and under $\$ 1$, a $30 \%$ increase from the previous time point might indicate touchscreen sensitivity issues rather than random responding. For example, a difference in responding of $2 \%$ likely to wait and use a condom at time point $\mathrm{X}$ and $4 \%$ likely to wait and use a condom at time point $\mathrm{Y}$ (a $50 \%$ increase) would be a violation of Criterion 1 but the absolute value difference between $2 \%$ and $4 \%$ is less relevant, and thus the criterion is overly sensitive when responses are in the $10 \%$ probability range of this task. This pattern of responding could indicate difficult interacting with the touchscreen and not, non-systematic responding which is what the algorithm is designed to detect. Criterion 1 is, however, still useful for identifying non-systematic data outside the $10 \%$ response area. To further verify this, visual inspection of curves were used for each participant and each condition. 
The second criterion can be used to identify when there is no change across the delays (Johnson \& Bickel, 2008). This response pattern would imply that the immediate reward has no value to the participant as the desire for it is not affected by delay (e.g., Criterion 2; Johnson \& Bickel, 2008). Johnson and Bickel (2008) propose that Criterion 2 violations are when the final time point is within $5 \%$ of the first time point. Due to the restricted temporal frame used in these tasks, it is possible that participants would not have preference shifts by the final time point (Stoltman et al., 2015), therefore this Criterion 2 was not used in the current study.

Non-systematic data was assessed for subgroup differences to determine if there are any underlying patterns. Figure 1 provides information about data orderliness for each Sexual Discounting Task condition. Figure 2 provides information about data orderliness across each Monetary Delay-Discounting Task condition.

Delay discounting data coding. AUC was used to compare the Monetary DelayDiscounting Task and Sexual Discounting Tasks. AUC is a standardized value ranging from 0-1 with a lower AUC value representing preference for smaller-sooner rewards (i.e., more discounting) than higher AUC values (Myerson, Green, \& Warusawitharana, 2001). AUC values are computed for each participant. To do so, the raw responses at each time point are converted to standardized responses (i.e., raw value/maximum larger-later value). These standardized values are then used to compute the area of the trapezoids that form the final AUC value (Figure 3). For example, at the delay of 2 minutes before a condom is available timepoint, a participant responded there is a $50 \%$ chance they would wait 2 minutes to use a condom. At the next delay, the participant responded there is a $40 \%$ chance they would wait 5 minutes to use a condom. These two responses would then be used to compute AUC for that portion of the curve (i.e., $[3 *[.4 / .5]] / 2)$. For the full formula, see Appendix D. 
Monetary Delay-Discounting Task AUC formula. When computing AUC for the Monetary Delay-Discounting Task, all cross-over points are transformed from their raw monetary amount to percent of the delayed, larger-later reward. For example, if a participant reports that in the $\$ 100$ condition at 60 minute delay they would prefer $\$ 85 \mathrm{NOW}$ as opposed to $\$ 100$ in 60 minutes, the value entered in the AUC equation (Appendix D) for the 60 minute delay would be $.85(85 / 100)$.

Sexual Discounting Task AUC formula. When computing AUC for the Sexual Discounting Task, all cross-over points are transformed from their raw percent condom preference and divided by $100 \%$, the larger-later amount. For example, if a participant reports that in the High Pregnancy Risk condition there is an $85 \%$ likelihood they would wait to use a condom at the 60 minute delay, the value entered in the AUC equation (Appendix D) for the 60 minute delay would be $0.85(85 \% / 100 \%)$.

An alpha of .05 was used for all statistical tests. All analyses were computed using SPSS v.25 (SPSS, Inc, Chicago, IL).

\section{Results}

\section{Demographics}

A total of 128 adults were enrolled in the study. However, 15 participants had incomplete data and were removed from the final analysis. Of those 15, two participants stopped the study before completing the Monetary Delay-Discounting Task (first discounting task assessed). Nine participants who completed the Sexual Discounting Task were not presented the Pregnancy Risk (Low) condition due to a randomization error in Qualtrics that was identified during the initial run of participants. Four participants did not complete Wave 1 and thus, never completed the questions that comprise the covariates. The final analytic sample includes 113 adults with 
complete data. The final analytic sample had a mean age of 34.3 years $(\mathrm{SD}=8.8$, range $19-69)$, with $73.5 \%$ self-identified as women, and $92.9 \%$ Caucasian. There were no significant differences observed between participants with complete, systematic data and participants with incomplete, non-systematic data on any of the relevant demographic comparisons. See Table 1 for full list of comparisons.

Self-reported past 12 month and lifetime condom use were low in this sample. In the past 12 months, $33.6 \%$ of participants reported using a condom $0 \%$ of the time, while $13.3 \%$ reported using a condom $100 \%$ of the time during this timeframe. When assessing lifetime condom use, $24.8 \%$ reported using condoms $1 \%$ - $10 \%$ of the time, while $5.3 \%$ reported using a condom $100 \%$ of the time. In contrast, a nationally representative sample of reproductive adults surveyed from $2011-2015$, found that $23.8 \%$ of reproductive-age women and $33.7 \%$ of reproductive-age men reported condom use at their during their last sexual intercourse (Copen, 2017).

Relationship status varied in the sample. Roughly a quarter of participants $(25.7 \%)$ reported never being married, while $24.8 \%$ are living with a partner, and $21.2 \%$ were married at the time of the survey with $91.2 \%$ reported having a sexual partner in the past year. The majority of participants have children $(78.8 \%)$. The current pregnancy rate was low in the sample at $19.3 \%$ of women. Roughly a quarter of women in the sample $(27.7 \%)$ reported being postmenopausal, had a hysterectomy, or both. Roughly $47 \%$ of the women in this sample cannot currently become pregnant either due to current pregnancy, being post-menopausal, having a hysterectomy or some combination of these three elements. To determine the role tangible pregnancy risk might have on responding, an analysis will include parsing out women at no risk of becoming pregnant at the time they were surveyed.

\section{Data orderliness}


Data normality. As shown in Table 2, the High STD Risk AUC and High Pregnancy

Risk AUC conditions were severely negatively skewed normality (z scores > 3.29; Field, 2013).

To determine if the skew is too high and needs to be transformed, the ratio of the skew divided by the standard error was examined. Due to the severity of the skew (Range: $-8.36--3.37$ ), logarithmic transformations were not sufficient to allow parametric distribution; therefore, nonparametric analysis was used. Table 2 provides the descriptives for all Sexual Discounting Task and Monetary Delay-Discounting Task data. All covariates met the assumptions of normality. Table 1 provides the descrpitives for all covariates.

Non-systematic data. Criterion 1 violations (i.e., a $\geq 30 \%$ increase between sequential timepoints) can indicate a lack of understanding the task, random responding, issues with the experimental conditions, or issues with the experimental environment that effected attention to the task. Therefore, participants with Criterion 1 violations did not have AUC computed for the conditions when the violations occurred (Johnson \& Bickel, 2008). In the High Attractiveness condition, 17 participants had Criterion 1 violations and 5 participants had multiple Criterion 1 violations within High Attractiveness. In the Low Attractiveness condition, 34 participants had Criterion 1 violations and 6 participants had multiple Criterion 1 violations within Low Attractiveness. In the High STD Risk condition, 20 participants had Criterion 1 violations and 5 participants had multiple Criterion 1 violations within High STD Risk. In the Low STD Risk condition, 22 participants had Criterion 1 violations and 7 participants had multiple Criterion 1 violations within Low STD Risk. In the High Pregnancy Risk condition, 12 participants had Criterion 1 violations and 2 participants had multiple Criterion 1 violations within High Pregnancy Risk. In the Low Pregnancy Risk condition, 9 participants had Criterion 1 violations and 5 participants had multiple Criterion 1 violations within Low Pregnancy Risk. Complete, 
systematic sexual delay discounting data was available for 48 participants ( $42 \%$ of the analytic sample). Sixty-five participants had at least one AUC imputed. Of the 65 participants with Criterion 1 violations, 41 had them in one condition and 24 had them in 2 or more conditions. Figure 1 includes a full accounting of Criterion 1 violations. Sample participant data with Criterion 1 violations are provided in Figure 4. A fully conditional specification method multiple imputations approach was used to compute AUC in conditions with non-systematic data. A fully conditional specification method multiple imputations approach uses Monte Carlo simulations to impute the missing data across multiple datasets prior to pooling the results during analysis (Liu \& De, 2015). All minimums were set to zero and maximums set to 1 .

Order effects. Three blocks were used to randomize the order discounting tasks were presented to participants: Block 1 for the monetary discounting had two levels; Block 2 for the original sexual discounting tasks (i.e., attraction and STD risk) had eight levels; and Block 3 for the new pregnancy risk task had two levels. Due to the number of randomization paths for Block 2, the overall cell sizes were small ranging from $10-18$ participants in each cell. See Appendix C for randomization procedures and exact cell sizes.

Order effects were observed for High Attraction $\left(\mathrm{F}_{(1,111)}=2.50 ; p=.020\right)$. A Tukey’s post-hoc analysis revealed that participants presented High Attraction, Low Attraction, Low STD Risk, High STD Risk had significantly lower High Attraction AUC compared to participants presented Low STD Risk, High STD Risk, High Attraction, Low Attraction. No other effects were observed for Low Attraction $(p=.223)$, High STD Risk $(p=.102)$, Low STD Risk $(p=$ .798), High Pregnancy Risk $(p=.503)$, Low Pregnancy Risk $(p=.053), \$ 10$ Monetary $(p=$ $.057)$, or $\$ 100$ Monetary $(p=.474)$. No other order effects were observed for the other blocks. 
The analytic sample. For the sake of parsimony and to maximize power, results are reported for the multiple imputations data set $(n=113)$. Full results are reported in Appendix E for participants with complete, systematic, non-zero "Immediately Available” Condom Preference data $(n=27)$.

\section{Data external validity check}

The original Sexual Discounting Task (Johnson \& Bruner, 2012) was used as a comparison with our sample on Attraction and STD Risk mean AUC. The Johnson and Bruner (2012) sample differed from ours in that their sample included non-treatment seeking cocaine dependent individuals while our sample was in-treatment for Opioid Use Disorder. However, few other sexual discounting studies included descriptives for the Sexual Discounting Task. A series of one sample $t$-tests were used to compare the Johnson and Bruner (2012) sample mean AUC and our sample mean AUC. The data for our sample were not significantly different from previous sexual discounting research in the High Attraction condition $t(112)=1.91, p=.156$, Low Attraction condition $t(112)=1.33, p=.749$, and High STD Risk condition $t(112)=1.20, p$ $=.721$. However, a significant difference was observed between participants in the analytic sample who had a significantly higher AUC in the Low STD Risk condition $\left(M_{A U C}=.52\right)$ compared to the Johnson and Bruner (2012) sample $\left(M_{A U C}=.44\right) ; t(112)=2.71, p=.044$.

Additionally, our sample did not have significantly different condom use attitudes when compared to not-in-treatment adults who reported use of cocaine and heroin (Rosengard, Anderson, \& Stein, 2006) regarding MCAS Identity Stigma Related to Condom Use, $t(112)=$ $.45, p=.657$, and MCAS Embarrassment About Negotiation and Use of Condoms, $t(111)=$ $1.73, p=.086$. However, a significant difference was observed between participants in the 
analytic sample that had significantly lower MCAS Condom Pleasure $(M=16.2)$ compared to the Rosengard et al. (2006) sample $(M=18.7) ; t(111)=-4.71, p<.001$.

Lastly, compared to a healthy adult sample in Brothers et al. (2014), participants in the analytic sample were not significantly different regarding Future Time Perspective in the Future as Limited domain, $t(112)=-.20, p=.843$. However, participants in the analytic sample had slightly lower perception of the Future as Open $(M=15.7)$ compared to the Brothers et al. (2014) sample $(M=16.6) ; t(112)=-3.22, p=.002$. Participants in the analytic sample also had significantly higher perception of the Future as Ambiguous $(M=11.8)$ compared to the Brothers et al. (2014) sample $(M=10.0) ; t(112)=5.30, p<.001$. Refer to Appendix F for comparisons with the present sample across these three tasks.

\section{Omnibus Testing}

A Friedman's non-parametric omnibus tests was computed to determine overall median differences in this repeated measure design. A comparison was computed between AUC for all the discounting conditions (i.e., High/Low Attraction, High/Low STD Risk, High/Low Pregnancy Risk). There was a statistically significant difference in AUC across conditions, $\chi^{2}(5)$ $=75.13, p<.001$. See Table 2 for AUC medians.

To test Research Question 1., are there domain differences in delay discounting?, a series of Wilcoxon Signed-rank Tests was computed between AUC Monetary Delay-Discounting Tasks and each AUC Sexual Discounting Task condition. See Table 3 for a full list of comparisons.

$\$ 10$ Monetary Delay-Discounting Task. The median AUC for the \$10 Monetary DelayDiscounting Task AUC $(\mathrm{Mdn}=.33, \mathrm{IQR}=.71)$ differed from the median Low Attraction AUC $(\mathrm{Mdn}=.73, \mathrm{IQR}=.65 ; \mathrm{Z}=-3.44, p=.002)$. Participants more steeply discounted the $\$ 10$ reward 
compared to condom choice in the Low Attraction condition. This response suggests a greater preference for smaller sooner rewards in the $\$ 10$ Monetary condition compared to a greater likelihood of waiting until a condom was available in the Low Attraction condition. See Table 3 for a full list of comparisons.

The median AUC for the $\$ 10$ Monetary Delay-Discounting Task AUC (Mdn = .33, IQR $=.71)$ differed from the median High STD Risk AUC $(\mathrm{Mdn}=1.00, \mathrm{IQR}=.41 ; \mathrm{Z}=-5.48$, $p<.001)$. Participants more steeply discounted the $\$ 10$ reward compared to condom choice in the High STD Risk condition. This response suggests a greater preference for smaller sooner rewards in the $\$ 10$ Monetary condition compared to a greater likelihood of waiting until a condom was available in the High STD Risk condition. See Table 3 for a full list of comparisons.

The median AUC for the $\$ 10$ Monetary Delay-Discounting Task AUC (Mdn $=.33$, IQR $=.71)$ differed from the median High Pregnancy Risk AUC $(\mathrm{Mdn}=1.00, \mathrm{IQR}=.43 ; \mathrm{Z}=$ $-5.41, p<.001)$. Participants more steeply discounted the $\$ 10$ reward compared to condom choice in the High Pregnancy Risk condition. This response suggests a greater preference for smaller sooner rewards in the $\$ 10$ Monetary condition compared to a greater likelihood of waiting until a condom was available in the High Pregnancy Risk condition. See Table 3 for a full list of comparisons.

The median AUC for the $\$ 10$ Monetary Delay-Discounting Task AUC (Mdn $=.33$, IQR $=.71)$ differed from the median Low Pregnancy Risk AUC $(\mathrm{Mdn}=.73, \mathrm{IQR}=.66 ; \mathrm{Z}=$ -2.97, $p=.014)$. Participants more steeply discounted the $\$ 10$ reward compared to condom choice in the Low Pregnancy Risk condition. This response suggests a greater preference for smaller sooner rewards in the $\$ 10$ Monetary condition compared to a greater likelihood of 
waiting until a condom was available in the Low Pregnancy Risk condition. See Table 3 for a full list of comparisons.

No significant differences in median AUC were detected between the \$10 Monetary Delay-Discounting Task and the High Attraction condition $(\mathrm{Z}=-0.54, p=.605)$. Additionally, no significant differences in median AUC were detected between the \$10 Monetary DelayDiscounting Task and the Low STD Risk condition $(Z=-1.12, p=.320)$. See Table 3 for a full list of comparisons.

$\$ 100$ Monetary Delay-Discounting Task. The median AUC for the \$100 Monetary Delay-Discounting Task AUC $(\mathrm{Mdn}=.63, \mathrm{IQR}=.62)$ differed from the median High Attraction AUC $(\mathrm{Mdn}=.51, \mathrm{IQR}=.86 ; \mathrm{Z}=-2.37, p=.019)$. Participants less steeply discounted the $\$ 100$ reward compared to condom choice in the High Attraction condition. This response suggests a less preference for smaller sooner rewards in the $\$ 100$ Monetary condition compared to a lower likelihood of waiting until a condom was available in the High Attraction condition. See Table 3 for a full list of comparisons.

The median AUC for the $\$ 100$ Monetary Delay-Discounting Task AUC (Mdn $=.63$, IQR $=.62)$ differed from the median High STD Risk AUC $(\mathrm{Mdn}=1.00, \mathrm{IQR}=.41 ; \mathrm{Z}=-3.53$, $p=.001)$. Participants more steeply discounted the $\$ 100$ reward compared to condom choice in the High STD Risk condition. This response suggests a greater preference for smaller sooner rewards in the $\$ 100$ Monetary condition compared to a greater likelihood of waiting until a condom was available in the High STD Risk condition. See Table 3 for a full list of comparisons.

The median AUC for the $\$ 100$ Monetary Delay-Discounting Task AUC (Mdn $=.63$, IQR $=.62)$ differed from the median High Pregnancy Risk AUC $(\mathrm{Mdn}=1.00, \mathrm{IQR}=.43 ; \mathrm{Z}=$ 
$-3.22, p=.003)$. Participants more steeply discounted the $\$ 100$ reward compared to condom choice in the High Pregnancy Risk condition. This response suggests a greater preference for smaller sooner rewards in the $\$ 100$ Monetary condition compared to a greater likelihood of waiting until a condom was available in the High Pregnancy Risk condition. See Table 3 for a full list of comparisons.

No significant differences in median AUC were detected between the $\$ 100$ Monetary Delay-Discounting Task and the Low Attraction condition $(\mathrm{Z}=-0.89, p=.453)$. Additionally, no significant differences in median AUC were detected between the \$100 Monetary DelayDiscounting Task and the Low STD Risk condition $(\mathrm{Z}=-1.50, p=.139)$. Lastly, no significant differences in median AUC were detected between the $\$ 100$ Monetary Delay-Discounting Task and the Low Pregnancy Risk condition $(Z=-0.38, p=.711)$. See Table 3 for a full list of comparisons.

High Attraction AUC. The median AUC for the High Attraction AUC (Mdn $=.51$, IQR $=.86)$ differed from the median High STD Risk AUC $(\mathrm{Mdn}=1.00, \mathrm{IQR}=.41$; $\mathrm{Z}=-5.51, p<.001)$. Participants more steeply discounted condoms in the High Attraction compared to the High STD Risk condition. This suggests there was a lower likelihood of waiting until a condom was available in the High Attraction condition compared to the High STD Risk condition. See Table 3 for a full list of comparisons.

The median AUC for the High Attraction AUC $(\mathrm{Mdn}=.51, \mathrm{IQR}=.86)$ differed from the median High Pregnancy Risk AUC $(\mathrm{Mdn}=1.00, \mathrm{IQR}=.43 ; \mathrm{Z}=-6.44, p<.001)$. Participants more steeply discounted condoms in the High Attraction compared to the High Pregnancy Risk condition. This suggests there was a lower likelihood of waiting until a condom was available in 
the High Attraction condition compared to the High Pregnancy Risk condition. See Table 3 for a full list of comparisons.

The median AUC for the High Attraction AUC $(\mathrm{Mdn}=.51, \mathrm{IQR}=.86)$ differed from the median Low Pregnancy Risk AUC $(\mathrm{Mdn}=.73, \mathrm{IQR}=.66 ; \mathrm{Z}=-4.00, p<.001)$. Participants more steeply discounted condoms in the High Attraction compared to the Low Pregnancy Risk condition. This suggests there was a lower likelihood of waiting until a condom was available in the High Attraction condition compared to the Low Pregnancy Risk condition. See Table 3 for a full list of comparisons.

No significant differences in median AUC were detected between the High Attraction condition and the Low STD Risk condition $(Z=-1.10, p=.293)$. See Table 3 for a full list of comparisons.

Low Attraction AUC. The median AUC for the Low Attraction AUC (Mdn = .73, IQR = $.65)$ differed from the median High STD Risk AUC (Mdn = 1.00, IQR = .41;

$\mathrm{Z}=-3.05, p=.009)$. Participants more steeply discounted condoms in the Low Attraction compared to the High STD Risk condition. This suggests there was a lower likelihood of waiting until a condom was available in the Low Attraction condition compared to the High STD Risk condition. See Table 3 for a full list of comparisons.

The median AUC for the Low Attraction AUC (Mdn = .73, IQR = .65) differed from the median Low STD Risk AUC $(\mathrm{Mdn}=.58, \mathrm{IQR}=.77 ; \mathrm{Z}=-2.68, p=.010)$. Participants less steeply discounted condoms in the Low Attraction condition compared to the Low STD Risk condition. This suggests there was a greater likelihood of waiting until a condom was available in the Low Attraction condition compared to the Low STD Risk condition. See Table 3 for a full list of comparisons. 
The median AUC for the Low Attraction AUC (Mdn = .73, IQR = .65) differed from the median and High Pregnancy Risk AUC $(\mathrm{Mdn}=1.00, \mathrm{IQR}=.43 ; \mathrm{Z}=-3.01, p=.030)$. Participants more steeply discounted condoms in the Low Attraction condition compared to the High Pregnancy Risk condition. This suggests there was a lower likelihood of waiting until a condom was available in the Low Attraction condition compared to the High Pregnancy Risk condition. See Table 3 for a full list of comparisons.

No significant differences in median AUC were detected between the Low Attraction condition and the Low Pregnancy Risk condition $(Z=-1.05, p=.415)$. See Table 3 for a full list of comparisons.

High STD Risk AUC. The median AUC for the High STD Risk AUC (Mdn = 1.00, IQR $=.41)$ differed from the median High Attraction $\mathrm{AUC}(\mathrm{Mdn}=.51, \mathrm{IQR}=.86$; $\mathrm{Z}=-5.51, p<.001)$. Participants less steeply discounted condoms in the High STD Risk condition compared to the High Attraction condition. This suggests there was a greater likelihood of waiting until a condom was available in the High STD Risk condition compared to the High Attraction condition. See Table 3 for a full list of comparisons.

The median AUC for High STD Risk AUC (Mdn = 1.00, IQR = .41) differed from the median the Low Attraction AUC $(\mathrm{Mdn}=.73, \mathrm{IQR}=.65 ; \mathrm{Z}=-3.05, p=.009)$. Participants less steeply discounted condoms in the High STD Risk condition compared to the Low Attraction condition. This suggests there was a greater likelihood of waiting until a condom was available in the High STD Risk condition compared to the Low Attraction condition. See Table 3 for a full list of comparisons.

The median AUC for the High STD Risk AUC (Mdn = 1.00, IQR = .41) differed from the median Low Pregnancy Risk AUC $(\mathrm{Mdn}=.73, \mathrm{IQR}=.66 ; \mathrm{Z}=-3.85, p<.001)$. Participants 
less steeply discounted condoms in the High STD Risk condition compared to the Low Pregnancy Risk condition. This suggests there was a greater likelihood of waiting until a condom was available in the High STD Risk condition compared to the Low Pregnancy Risk condition. See Table 3 for a full list of comparisons.

No significant differences in median AUC were detected between High STD Risk condition and the High Pregnancy Risk condition $(\mathrm{Z}=-0.50, p=.647)$. See Table 3 for a full list of comparisons.

Low STD Risk AUC. The median AUC for the Low STD Risk AUC (Mdn = .58, IQR = .77) differed from the median Low Attraction AUC (Mdn $=.73, \mathrm{IQR}=.65 ; \mathrm{Z}=-2.68, \mathrm{p}=.010)$. Participants more steeply discounted condoms in the Low STD Risk condition compared to the Low Attraction condition. This suggests there was a lower likelihood of waiting until a condom was available in the Low STD Risk condition compared to the Low Attraction condition. See Table 3 for a full list of comparisons.

The median AUC for the Low STD Risk AUC (Mdn = .58, IQR = .77) differed from the median High Pregnancy Risk AUC $(\mathrm{Mdn}=1.00, \mathrm{IQR}=.43 ; \mathrm{Z}=-5.47, p<.001)$. Participants more steeply discounted condoms in the Low STD Risk condition compared to the High Pregnancy Risk condition. This suggests there was a lower likelihood of waiting until a condom was available in the Low STD Risk condition compared to the High Pregnancy Risk condition. See Table 3 for a full list of comparisons.

The median AUC for the Low STD Risk AUC (Mdn =.58, IQR = .77) differed from the median Low Pregnancy Risk AUC (Mdn = .73, IQR = .66; Z = -2.88, $p=.030)$. Participants more steeply discounted condoms in the Low STD Risk condition compared to the Low Pregnancy Risk condition. This suggests there was a lower likelihood of waiting until a condom 
was available in the Low STD Risk condition compared to the Low Pregnancy Risk condition. See Table 3 for a full list of comparisons.

No significant differences in median AUC were detected between Low STD Risk condition and the High Attraction condition $(Z=-1.10, p=.293)$. See Table 3 for a full list of comparisons.

High Pregnancy Risk AUC. The median AUC for the High Pregnancy Risk AUC (Mdn $=1.00, \mathrm{IQR}=.43)$ differed from the median High Attraction $\mathrm{AUC}(\mathrm{Mdn}=.51, \mathrm{IQR}=.86 ; \mathrm{Z}=-$ $6.44, p<.001)$. Participants more less steeply discounted condoms in the High Pregnancy Risk condition compared to the High Attraction condition. This suggests there was a greater likelihood of waiting until a condom was available in the High Pregnancy Risk condition compared to the High Attraction condition. See Table 3 for a full list of comparisons.

The median AUC for the High Pregnancy Risk AUC $(\mathrm{Mdn}=1.00, \mathrm{IQR}=.43)$ differed from the median and Low Attraction AUC $(\mathrm{Mdn}=.73, \mathrm{IQR}=.65 ; \mathrm{Z}=-3.01, p=.030)$. Participants less steeply discounted condoms in the High Pregnancy Risk condition compared to the Low Attraction condition. This suggests there was a greater likelihood of waiting until a condom was available in the High Pregnancy Risk condition compared to the Low Attraction condition. See Table 3 for a full list of comparisons.

The median AUC for the High Pregnancy Risk AUC $(\mathrm{Mdn}=1.00, \mathrm{IQR}=.43)$ differed from the median Low STD Risk AUC $(\mathrm{Mdn}=.58, \mathrm{IQR}=.77 ; \mathrm{Z}=-5.47, p<.001)$. Participants less steeply discounted condoms in the High Pregnancy Risk condition compared to the Low STD Risk condition. This suggests there was a greater likelihood of waiting until a condom was available in the High Pregnancy Risk condition compared to the Low STD Risk condition. See Table 3 for a full list of comparisons. 
No significant differences in median AUC were detected between the High Pregnancy Risk condition and the High STD Risk condition $(Z=-0.50, p=.647)$. See Table 3 for a full list of comparisons.

Low Pregnancy Risk AUC. The median AUC for the Low Pregnancy Risk AUC (Mdn = $.73, \mathrm{IQR}=.66)$ differed from the median High Attraction AUC $(\mathrm{Mdn}=.51, \mathrm{IQR}=.86 ; \mathrm{Z}=-$ 4.00, $p<.001)$. Participants less steeply discounted condoms in the Low Pregnancy Risk compared to the High Attraction condition. This suggests there was a greater likelihood of waiting until a condom was available in the Low Pregnancy Risk condition compared to the High Attraction condition. See Table 3 for a full list of comparisons.

The median AUC for the Low Pregnancy Risk AUC (Mdn = .73, IQR = .66) differed from the median High STD Risk AUC $(\mathrm{Mdn}=1.00, \mathrm{IQR}=.41 ; \mathrm{Z}=-3.85$, $p<.001)$. Participants more steeply discounted condoms in the Low Pregnancy Risk condition compared to the High STD Risk condition. This suggests there was a lower likelihood of waiting until a condom was available in the Low Pregnancy Risk condition compared to the High STD Risk condition. See Table 3 for a full list of comparisons.

The median AUC for the Low Pregnancy Risk AUC $(\mathrm{Mdn}=.73, \mathrm{IQR}=.66)$ differed from the median Low STD Risk AUC (Mdn =.58, IQR = .77; Z = -2.88, $p=.030)$. Participants less steeply discounted condoms in the Low Pregnancy Risk condition compared to the Low STD Risk condition. This suggests there was a greater likelihood of waiting until a condom was available in the Low Pregnancy Risk condition compared to the Low STD Risk condition. See Table 3 for a full list of comparisons. 
No significant differences in median AUC were detected between the Low Pregnancy Risk condition and the Low Attraction condition $(Z=-1.05, p=.415)$. See Table 3 for a full list of comparisons.

To test Research Question 2., Does reward magnitude influence choice for monetary and sexual discounting, a series of Wilcoxon Signed-rank Tests was computed between AUCs within the Monetary Delay-Discounting Task and within each Sexual Discounting Task condition. See Table 3 for full list of comparisons. See Figure 5 for visual depiction of $\$ 10$ and $\$ 100$ Monetary percent of immediate value chosen across delays. See Figure 6 for a visual depiction of percent likelihood of condom use by delay in each condition.

RQ2a: Within Condition Comparison for Monetary Delay-Discounting Task AUC. In order to examine magnitude differences in delay discounting, comparisons were computed within the Monetary Delay-Discounting Task between $\$ 10$ and $\$ 100$ conditions. The median AUC for the $\$ 10$ Monetary condition $(\mathrm{Mdn}=.33, \mathrm{IQR}=.71)$ differed from the median AUC for the $\$ 100$ Monetary condition $(\mathrm{Mdn}=.63, \mathrm{IQR}=.62 ; \mathrm{Z}=-4.41, p<.001)$. Participants more steeply discounted money in the $\$ 10$ Monetary condition compared to the $\$ 100$ Monetary condition. This indicates a greater preference for smaller sooner rewards in the $\$ 10$ Monetary condition compared to the $\$ 100$ Monetary condition. See Table 3 for a full list of comparisons. See Figure 5 for visual depiction of $\$ 10$ and $\$ 100$ Monetary percent of immediate value chosen across delays.

\section{RQ2b: Within Condition Comparison for All Raw Sexual Discounting Task AUC.}

In order to examine magnitude differences in delay discounting, comparisons were computed between AUCs within each Sexual Discounting Task condition. See Table 3 for full list of 
comparisons. See Figure 6 for a visual depiction of percent likelihood of condom use by delay in each condition.

Attraction AUC. The median AUC for the High Attraction condition (Mdn $=.51, \mathrm{IQR}=$ .86) differed from the median AUC for the Low Attraction condition (Mdn $=.73$, IQR $=.65 ; \mathrm{Z}=$ -4.03, $p<.001)$. Participants more steeply discounted condom use in the High Attraction condition compared to the Low Attraction condition. This indicates there was a lower likelihood of waiting until a condom was available in the High Attraction condition compared to the Low Attraction condition. See Table 3 for full list of comparisons. See Figure 6 for a visual depiction of percent likelihood of condom use by delay in each condition.

STD Risk AUC. The median AUC for the High STD Risk condition (Mdn = 1.00, IQR = .41) differed from the median AUC for the Low STD Risk condition $(\mathrm{Mdn}=.58, \mathrm{IQR}=.77 ; \mathrm{Z}=$ $-5.39, p<.001)$. Participants less steeply discounted condom use in the High STD Risk condition compared to the Low STD Risk condition. This indicates there was a greater likelihood of waiting until a condom was available in the High STD Risk condition compared to the Low STD Risk condition. See Table 3 for full list of comparisons. See Figure 6 for a visual depiction of percent likelihood of condom use by delay in each condition.

Pregnancy Risk AUC. The median AUC for the High Pregnancy Risk condition (Mdn = $1.00, \mathrm{IQR}=.43)$ differed from the median AUC for the Low Pregnancy Risk condition $(\mathrm{Mdn}=$ $.73, \mathrm{IQR}=.66 ; \mathrm{Z}=-3.55, p<.001)$. Participants less steeply discounted condom use in the High Pregnancy Risk condition compared to the Low STD Pregnancy condition. This indicates there was a greater likelihood of waiting until a condom was available in the High Pregnancy Risk condition compared to the Low Pregnancy Risk condition. See Table 3 for full list of 
comparisons. See Figure 6 for a visual depiction of percent likelihood of condom use by delay in each condition.

To test Research Question 3a, are differences in sexual discounting associate with individual differences, a Spearman rank correlations were computed using Future Time Perspective, MCAS Condom Attitudes, Sexual Arousal, and AUC for each Sexual Discounting Task condition. A Mann-Whitney U was used due to non-parametric data to test median differences in AUC between median splits for each covariate. AUC is on a $0-1$ scale whereby lower AUC indicates less preference for condoms across delays. See Table 4 for the full list of comparisons.

Future Time Perspective. Future Time Perspective was assessed using a brief, 12-item measure (Brothers et al., 2014). Future Time Perspective was categorized into three subfactors: Future as Open, Future as Limited, and Future as Ambiguous (Brothers et al., 2014). Each subscale is discussed below.

Future as Open. No significant correlations were observed between Future as Open and AUC across the Sexual Discounting Task conditions: High Attraction $\mathrm{r}_{\mathrm{s}}(111)=-.007$, Low Attraction $\mathrm{r}_{\mathrm{s}}(111)=.105$, High STD Risk $\mathrm{r}_{\mathrm{s}}(111)=.077$, Low STD Risk $\mathrm{r}_{\mathrm{s}}(111)=-.006$, High Pregnancy Risk $\mathrm{r}_{\mathrm{s}}(111)=-.107$, or Low Pregnancy Risk $\mathrm{r}_{\mathrm{s}}(111)=.033$. See Table 4 for the full list of comparisons.

Future as Limited. Future as Limited was significantly negatively correlated with High Pregnancy Risk AUC, $\mathrm{r}_{\mathrm{S}}(111)=-.245, p<.01$. That is, a lower likelihood of waiting until a condom was available in the High Pregnancy Risk condition was associated with a higher endorsement of the Future as Limited. Conversely, a greater likelihood of waiting until a condom 
was available in the High Pregnancy Risk condition was associated with a lower endorsement of the Future as Limited. See Table 4 and Table 5 for the full list of comparisons.

Future as Limited was significantly negatively correlated with Low Pregnancy Risk Raw AUC, $\mathrm{r}_{\mathrm{s}}(111)=-.219, p<.05$. That is, a lower likelihood of waiting until a condom was available in the Low Pregnancy Risk condition was associated with a higher endorsement of the Future as Limited. Conversely, a greater likelihood of waiting until a condom was available in the Low Pregnancy Risk condition was associated with a lower endorsement of the Future as Limited. See Table 4 and Table 5 for the full list of comparisons.

No significant correlations were observed between Future as Limited and AUC across the Sexual Discounting Task conditions: High Attraction $r_{\mathrm{s}}(111)=-.148$, Low Attraction $r_{\mathrm{s}}(111)=$ -.073 , High STD Risk $r_{\mathrm{s}}(111)=.005$, or Low STD Risk $\mathrm{r}_{\mathrm{s}}(111)=-.157$. No significant differences were observed between High/Low Future as Limited on any AUC across the Sexual Discounting Task conditions: High Attraction, Low Attraction, High STD Risk, Low STD Risk, High Pregnancy Risk, or Low Pregnancy Risk. See Table 4 and Table 5 for the full list of comparisons.

Future as Ambiguous. Future as Ambiguous was significantly negatively correlated with Low Attraction AUC, $\mathrm{r}_{\mathrm{s}}(111)=-.208, p<.05$. That is, a lower likelihood of waiting until a condom was available in the Low Attraction condition was associated with a higher endorsement of the Future as Ambiguous. Conversely, a greater likelihood of waiting until a condom was available in the Low Attraction condition was associated with a lower endorsement of the Future as Ambiguous. See Table 4 and Table 6 for the full list of comparisons.

Future as Ambiguous was significantly negatively correlated with Low Pregnancy Risk AUC, $\mathrm{r}_{\mathrm{s}}(111)=-.232, p<.05$. That is, a lower likelihood of waiting until a condom was 
available in the Low Pregnancy Risk condition was associated with a higher endorsement of the Future as Ambiguous. Conversely, a greater likelihood of waiting until a condom was available in the Low Pregnancy Risk condition was associated with a lower endorsement of the Future as Ambiguous. See Table 4 and Table 6 for the full list of comparisons.

To probe this correlation between Future as Ambiguous and the Low Pregnancy Risk condition further, a median split was completed to separate Future as Ambiguous into two groups. Due to the non-parametric properties found in the AUC data, a Mann-Whitney U was used to test median AUC differences between High Future as Ambiguous and Low Future as Ambiguous. High Future as Ambiguous had significantly lower AUC (Mdn = .59, IQR = .70) compared to Low Future as Ambiguous $(\mathrm{Mdn}=.86, \mathrm{IQR}=.53 ; \mathrm{U}=1100.2, p=.015)$ in the Low Pregnancy Risk condition. Participants with High Future as Ambiguous had a lower likelihood of waiting until a condom was available compared with Low Future as Ambiguous in the Low Pregnancy Risk condition. See Table 4 and Table 6 for the full list of comparisons.

No significant correlations were observed between Future as Ambiguous and AUC across the Sexual Discounting Task conditions: High Attraction $\mathrm{r}_{\mathrm{s}}(111)=-.170$, High STD Risk $\mathrm{r}_{\mathrm{s}}(111)$ $=-.088$, Low STD Risk $\mathrm{r}_{\mathrm{s}}(111)=-.127$, or High Pregnancy Risk $\mathrm{r}_{\mathrm{s}}(111)=-.047$. No other significant differences were observed between High/Low Future as Ambiguous on AUC across the Sexual Discounting Task conditions: High Attraction, Low Attraction, High STD Risk, Low STD Risk, or High Pregnancy Risk. See Table 4 and Table 6 for the full list of comparisons.

MCAS. Condom attitudes were assessed using The UCLA Multidimensional Condom Attitude Scale (MCAS; Helweg-Larsen \& Collins, 1994). The MCAS was categorized into three subfactors: Condom Pleasure, Identity Stigma Related to Condom Use, Embarrassment About 
Negotiation and Use of Condoms (Helweg-Larsen \& Collins, 1994). Each is discussed below. See Table 4 for the full list of comparisons.

MCAS Condom Pleasure. MCAS Condom Pleasure was significantly positively correlated with High Attraction AUC, $\mathrm{r}_{\mathrm{s}}(111)=.367, p<.01$. That is, a greater likelihood of waiting until a condom was available in the High Attraction condition was associated with a higher endorsement of the condoms as pleasurable. Conversely, a lower likelihood of waiting until a condom was available in the High Attraction condition was associated with a lower endorsement of the condoms as pleasurable. See Table 4 and Table 7 for the full list of comparisons.

MCAS Condom Pleasure was significantly positively correlated with Low STD Risk AUC, $\mathrm{r}_{\mathrm{s}}(111)=.270, p<.01$. That is, a greater likelihood of waiting until a condom was available in the Low STD Risk condition was associated with a higher endorsement of the condoms as pleasurable. Conversely, a lower likelihood of waiting until a condom was available in the Low STD Risk condition was associated with a lower endorsement of the condoms as pleasurable. See Table 4 and Table 7 for the full list of comparisons.

MCAS Condom Pleasure was significantly positively correlated with Low Pregnancy Risk AUC, $\mathrm{r}_{\mathrm{s}}(111)=.360, p<.01$. That is, a greater likelihood of waiting until a condom was available in the Low Pregnancy Risk condition was associated with a higher endorsement of the condoms as pleasurable. Conversely, a lower likelihood of waiting until a condom was available in the Low Pregnancy Risk condition was associated with a lower endorsement of the condoms as pleasurable. See Table 4 and Table 7 for the full list of comparisons.

To probe this correlation between MCAS Condom Pleasure and the High Attraction condition further, a median split was completed to separate MCAS Condom Pleasure into two 
groups. Due to the non-parametric properties found in the AUC data, a Mann-Whitney U was used to test median AUC differences between High MCAS Condom Pleasure and Low MCAS Condom Pleasure. High MCAS Condom Pleasure had significantly higher AUC (Mdn = .71, $\mathrm{IQR}=.64)$ compared to Low MCAS Condom Pleasure $(\mathrm{Mdn}=.24, \mathrm{IQR}=.71 ; \mathrm{U}=844.7, p<$ $.001)$ in the High Attraction condition. Participants with High MCAS Condom Pleasure had a greater likelihood of waiting until a condom was available in the High Attraction condition compared with Low MCAS Condom Pleasure. See Table 4 and Table 7 for the full list of comparisons.

To probe this correlation between MCAS Condom Pleasure and the Low STD Risk condition further, a median split was completed to separate MCAS Condom Pleasure into two groups. Due to the non-parametric properties found in the AUC data, a Mann-Whitney U was used to test median AUC differences between High MCAS Condom Pleasure and Low MCAS Condom Pleasure. High MCAS Condom Pleasure had significantly higher AUC (Mdn = .70, $\mathrm{IQR}=.63)$ compared to Low MCAS Condom Pleasure $(\mathrm{Mdn}=.38, \mathrm{IQR}=.88 ; \mathrm{U}=1015.2, p=$ .006) in the Low STD Risk condition. Participants with High MCAS Condom Pleasure had a greater likelihood of waiting until a condom was available in the Low STD Risk condition compared to Low MCAS Condom Pleasure. See Table 4 and Table 7 for the full list of comparisons.

To probe this correlation between MCAS Condom Pleasure and the Low Pregnancy Risk condition further, a median split was completed to separate MCAS Condom Pleasure into two groups. Due to the non-parametric properties found in the AUC data, a Mann-Whitney U was used to test median AUC differences between High MCAS Condom Pleasure and Low MCAS Condom Pleasure. High MCAS Condom Pleasure had significantly higher AUC (Mdn = .87, 
$\mathrm{IQR}=.42)$ compared to Low MCAS Condom Pleasure $(\mathrm{Mdn}=.58, \mathrm{IQR}=.83 ; \mathrm{U}=1038.7, p=$ .004) in the Low Pregnancy Risk condition. Participants with High MCAS Condom Pleasure had a greater likelihood of waiting until a condom was available in the Low Pregnancy Risk condition compared to Low MCAS Condom Pleasure. See Table 4 and Table 7 for the full list of comparisons.

No significant correlations were observed between MCAS Pleasure and AUC across the Sexual Discounting Task conditions: Low Attraction $r_{\mathrm{s}}(111)=.146$, High STD Risk $\mathrm{r}_{\mathrm{s}}(111)=$ -.022, or High Pregnancy Risk $\mathrm{r}_{\mathrm{s}}(111)=.179$. No other significant differences were observed between High/Low MCAS Condom Pleasure on AUC across the Sexual Discounting Task conditions: Low Attraction, High STD Risk, or High Pregnancy Risk. See Table 4 and Table 7 for the full list of comparisons.

MCAS Identity Stigma Related to Condom Use. MCAS Identity Stigma Related to Condom Use was significantly positively correlated with High STD Risk AUC, $\mathrm{r}_{\mathrm{s}}(111)=.413, p$ $<.01$. That is, a greater likelihood of waiting until a condom was available in the High STD Risk condition was associated with a higher endorsement of a stigma attached to people who use condoms. Conversely, a lower likelihood of waiting until a condom was available in the High STD Risk condition was associated with a lower endorsement of a stigma attached to people who use condoms. See Table 4 and Table 8 for the full list of comparisons.

MCAS Identity Stigma Related to Condom Use was significantly positively correlated with High Pregnancy Risk AUC, $\mathrm{r}_{\mathrm{s}}(111)=.215, p<.05$. That is, a greater likelihood of waiting until a condom was available in the High Pregnancy Risk condition was associated with a higher endorsement of a stigma attached to people who use condoms. Conversely, a lower likelihood of waiting until a condom was available in the High Pregnancy Risk condition was associated with 
a lower endorsement of a stigma attached to people who use condoms. See Table 4 and Table 8 for the full list of comparisons.

MCAS Identity Stigma Related to Condom Use was significantly positively correlated with Low Pregnancy Risk AUC, $\mathrm{r}_{\mathrm{s}}(111)=.262, p<.01$. That is, a greater likelihood of waiting until a condom was available in the Low Pregnancy Risk condition was associated with a higher endorsement of a stigma attached to people who use condoms. Conversely, a lower likelihood of waiting until a condom was available in the Low Pregnancy Risk condition was associated with a lower endorsement of a stigma attached to people who use condoms. See Table 4 and Table 8 for the full list of comparisons.

To probe this correlation between MCAS Identity Stigma Related to Condom Use and the High Attraction condition further, a median split was completed to separate MCAS Identity Stigma Related to Condom Use into two groups. Due to the non-parametric properties found in the AUC data, a Mann-Whitney U was used to test median AUC differences between High MCAS Identity Stigma Related to Condom Use and Low MCAS Identity Stigma Related to Condom Use. High MCAS Identity Stigma Related to Condom Use had significantly higher AUC $(\mathrm{Mdn}=.60, \mathrm{IQR}=.82)$ compared to Low MCAS Identity Stigma Related to Condom Use $(\mathrm{Mdn}=.36, \mathrm{IQR}=.78 ; \mathrm{U}=1160.7, p=.041)$ in the High Attraction condition. Participants with High MCAS Identity Stigma Related to Condom Use had a greater likelihood of waiting until a condom was available in the High Attraction condition compared to Low MCAS Identity Stigma Related to Condom Use. See Table 4 and Table 8 for the full list of comparisons.

To probe this correlation between MCAS Identity Stigma Related to Condom Use and the High Risk STD condition further, a median split was completed to separate MCAS Identity Stigma Related to Condom Use into two groups. Due to the non-parametric properties found in 
the AUC data, a Mann-Whitney U was used to test median AUC differences between High MCAS Identity Stigma Related to Condom Use and Low MCAS Identity Stigma Related to Condom Use. High MCAS Identity Stigma Related to Condom Use had significantly higher AUC $(\mathrm{Mdn}=1.00, \mathrm{IQR}=.01)$ compared to Low MCAS Identity Stigma Related to Condom Use $(\mathrm{Mdn}=.80, \mathrm{IQR}=.60 ; \mathrm{U}=925.2, p=.001)$ in the High STD Risk condition. Participants with High MCAS Identity Stigma Related to Condom Use had a greater likelihood of waiting until a condom was available in the High STD Risk condition compared to Low MCAS Identity Stigma Related to Condom Use. See Table 4 and Table 8 for the full list of comparisons.

Due to the non-parametric properties found in the AUC data, a Mann-Whitney U was used to test median AUC differences between High MCAS Identity Stigma Related to Condom Use and Low MCAS Identity Stigma Related to Condom Use. High MCAS Identity Stigma Related to Condom Use had significantly higher AUC $(\mathrm{Mdn}=.70, \mathrm{IQR}=.71)$ compared to Low MCAS Identity Stigma Related to Condom Use $(\mathrm{Mdn}=.39, \mathrm{IQR}=.72 ; \mathrm{U}=1157.3, p=.049)$ in the Low STD Risk condition. Participants with High MCAS Identity Stigma Related to Condom Use had a greater likelihood of waiting until a condom was available in the Low STD Risk condition compared to Low MCAS Identity Stigma Related to Condom Use. See Table 4 and Table 8 for the full list of comparisons.

To probe this correlation between MCAS Identity Stigma Related to Condom Use and the High Pregnancy Risk condition further, a median split was completed to separate MCAS Identity Stigma Related to Condom Use into two groups. Due to the non-parametric properties found in the AUC data, a Mann-Whitney U was used to test median AUC differences between High MCAS Identity Stigma Related to Condom Use and Low MCAS Identity Stigma Related to Condom Use. High MCAS Identity Stigma Related to Condom Use had significantly higher 
AUC $(\mathrm{Mdn}=1.00, \mathrm{IQR}=.25)$ compared to Low MCAS Identity Stigma Related to Condom Use $(\mathrm{Mdn}=.88, \mathrm{IQR}=.65 ; \mathrm{U}=1120.2, p=.012)$ in the High Pregnancy Risk condition. Participants with High MCAS Identity Stigma Related to Condom Use had a greater likelihood of waiting until a condom was available in the High Pregnancy Risk condition compared to Low MCAS Identity Stigma Related to Condom Use. See Table 4 and Table 8 for the full list of comparisons.

To probe this correlation between MCAS Identity Stigma Related to Condom Use and the Low Pregnancy Risk condition further, a median split was completed to separate MCAS Identity Stigma Related to Condom Use into two groups. Due to the non-parametric properties found in the AUC data, a Mann-Whitney U was used to test median AUC differences between High MCAS Identity Stigma Related to Condom Use and Low MCAS Identity Stigma Related to Condom Use. High MCAS Identity Stigma Related to Condom Use had significantly higher AUC $(\mathrm{Mdn}=.89, \mathrm{IQR}=.46)$ compared to Low MCAS Identity Stigma Related to Condom Use $(\mathrm{Mdn}=.53, \mathrm{IQR}=.74 ; \mathrm{U}=1048.0, p=.004)$ in the Low Pregnancy Risk condition. Participants with High MCAS Identity Stigma Related to Condom Use had a greater likelihood of waiting until a condom was available in the Low Pregnancy Risk condition compared to Low MCAS Identity Stigma Related to Condom Use. See Table 4 and Table 8 for the full list of comparisons.

No significant correlations were observed between MCAS Identity Stigma Related to Condom Use and AUC across the Sexual Discounting Task conditions: High Attraction $\mathrm{r}_{\mathrm{s}}(111)$ $=.150$, Low Attraction $\mathrm{r}_{\mathrm{s}}(111)=.151$, or Low STD Risk $\mathrm{r}_{\mathrm{s}}(111)=.166$. No other significant differences were observed between High/Low MCAS Condom Pleasure on AUC across the Low Attraction Sexual Discounting Task conditions. See Table 4 and Table 8 for the full list of comparisons. 
MCAS Embarrassment About Negotiation and Use of Condoms. MCAS Embarrassment About Negotiation and Use of Condoms was significantly positively correlated with Low Attraction AUC, $\mathrm{r}_{\mathrm{s}}(111)=.199, p<.05$. That is, a greater likelihood of waiting until a condom was available in the Low Attraction condition was associated with a higher endorsement of embarrassment about negotiating and using condoms. Conversely, a lower likelihood of waiting until a condom was available in the Low Attraction condition was associated with a lower endorsement of embarrassment about negotiating and using condoms. See Table 4 and Table 9 for the full list of comparisons.

MCAS Embarrassment About Negotiation and Use of Condoms was significantly positively correlated with High Pregnancy Risk AUC, $\mathrm{r}_{\mathrm{s}}(111)=.213, p<.05$. That is, a greater likelihood of waiting until a condom was available in the High Pregnancy Risk condition was associated with a higher endorsement of embarrassment about negotiating and using condoms. Conversely, a lower likelihood of waiting until a condom was available in the Low Attraction condition was associated with a lower endorsement of embarrassment about negotiating and using condoms. See Table 4 and Table 9 for the full list of comparisons.

No significant correlations were observed between MCAS Embarrassment About Negotiation and Use of Condoms and AUC across the Sexual Discounting Task conditions: High Attraction $r_{\mathrm{s}}(111)=.118$, High STD Risk $\mathrm{r}_{\mathrm{s}}(111)=.166$, Low STD Risk $\mathrm{r}_{\mathrm{s}}(111)=.001$, or Low Pregnancy Risk $\mathrm{r}_{\mathrm{s}}(111)=.053$. No significant differences were observed between High/Low MCAS Embarrassment About Negotiation and Use of Condoms on AUC across the Sexual Discounting Task conditions: High Attraction, Low Attraction, High STD Risk, Low STD Risk, High Pregnancy Risk, or Low Pregnancy Risk. See Table 4 and Table 9 for the full list of comparisons. 
Sexual Arousal. Sexual Arousal was significantly negatively correlated with High Pregnancy Risk AUC, $\mathrm{r}_{\mathrm{s}}(111)=-.281, p<.01$. That is, a lower likelihood of waiting until a condom was available in the High Pregnancy Risk condition was associated with a higher sexual arousal. Conversely, a greater likelihood of waiting until a condom was available in the High Pregnancy Risk condition was associated with a lower sexual arousal. See Table 4 and Table 10 for the full list of comparisons.

To probe this correlation between Sexual Arousal and the High Pregnancy Risk condition further, a median split was completed to separate Sexual Arousal into two groups. Due to the non-parametric properties found in the AUC data, a Mann-Whitney U was used to test median AUC differences between High Sexual Arousal and Low Sexual Arousal. High Sexual Arousal had significantly lower AUC $(\mathrm{Mdn}=.88, \mathrm{IQR}=.58)$ compared to Low Sexual Arousal $(\mathrm{Mdn}=$ $1.00, \mathrm{IQR}=.28 ; \mathrm{U}=1178.8, p=.029)$ in the High Pregnancy Risk condition. Participants with High Sexual Arousal had a lower likelihood of waiting until a condom was available in the High Pregnancy Risk condition compared to Low Sexual Arousal. See Table 4 and Table 10 for the full list of comparisons.

No significant correlations were observed between sexual arousal and AUC across the Sexual Discounting Task conditions: High Attraction, Low Attraction, High STD Risk, Low STD Risk, or Low Pregnancy Risk. No significant differences were observed between High/Low Sexual Arousal on AUC across the Sexual Discounting Task conditions: High Attraction $\mathrm{r}_{\mathrm{s}}(111)$ $=-.138$, Low Attraction $r_{\mathrm{s}}(111)=-.071$, High STD Risk $\mathrm{r}_{\mathrm{s}}(111)=-.156$, Low STD Risk $\mathrm{r}_{\mathrm{s}}(111)$ $=-.118$, or Low Pregnancy Risk $\mathrm{r}_{\mathrm{s}}(111)=-.143$. See Table 4 and Table 10 for the full list of comparisons. 
Due to the non-parametric properties found in the AUC data, a Mann-Whitney U was used to test Research Question 3b.: Are differences in sexual discounting associated with gender, median AUC was compared between genders for each the Sexual Discounting Task condition (Gender X Attractiveness, Gender X STD Risk, Gender X Pregnancy Risk). See Table 7 for the full list of gender comparisons.

Gender. No significant difference was observed between gender and AUC across the Sexual Discounting Task conditions: High Attraction $U=1024.0, p=.307$, Low Attraction $U=$ 927.0, $p=.220$, High STD Risk $U=1084.3, p=.500$, Low STD Risk $U=960.2, p=.176$, High Pregnancy Risk $U=951.2, p=.096$, or Low Pregnancy Risk $U=940.2, p=.085$. See Table 11 for the full list of gender comparisons.

Age. Age was significantly positively correlated with High Attraction AUC, $\mathrm{r}_{\mathrm{s}}(111)=$ $.198, p<.05$. That is, a lower likelihood of waiting until a condom was available in the High Attraction condition was associated with a lower age. Conversely, a greater likelihood of waiting until a condom was available in the High Attraction condition was associated with a higher age. See Table 4 for the full list of comparisons.

Age was significantly positively correlated with Low STD Risk AUC, $\mathrm{r}_{\mathrm{s}}(111)=.222, p<$ .05. That is, a lower likelihood of waiting until a condom was available in the Low STD Risk condition was associated with a lower age. Conversely, a greater likelihood of waiting until a condom was available in the Low STD Risk condition was associated with a higher age. See Table 4 for the full list of comparisons.

Age was significantly positively correlated with High Pregnancy Risk AUC, $\mathrm{r}_{\mathrm{s}}(111)=$ $.232, p<.05$. That is, a lower likelihood of waiting until a condom was available in the High Pregnancy Risk condition was associated with a lower age. Conversely, a greater likelihood of 
waiting until a condom was available in the High Pregnancy Risk condition was associated with a higher age. See Table 4 for the full list of comparisons.

No significant correlations were observed between Age and various Sexual Discounting Task conditions including: Low Attraction $\mathrm{r}_{\mathrm{s}}(111)=.063$, High STD Risk $\mathrm{r}_{\mathrm{s}}(111)=.014$, or Low Pregnancy Risk $\mathrm{r}_{\mathrm{s}}(111)=.142$. See Table 4 for the full list of comparisons.

Reproductive-age adults. A Kruskal-Wallis non-parametric omnibus test was computed to determine overall median differences between reproductive-aged men, reproductive-aged women, currently pregnant women, and women not at risk for a pregnancy (e.g., women who reported having a hysterectomy, being post-menopausal, or both) across all Sexual Discounting conditions. The Kruskal-Wallis H test was statistically significant for the Low STD Risk condition $\chi^{2}(3)=8.65, p=.034$ and the Low Pregnancy Risk condition $\chi^{2}(3)=10.42, p=.015$. Post-hoc pairwise comparisons revealed that women who had a hysterectomy, are postmenopausal, or both, had greater likelihood of waiting until a condom was available in the Low STD Risk condition when compared to reproductive-aged men $(p=.042)$. An additional posthoc pairwise comparisons revealed that women who had a hysterectomy, are post-menopausal, or both, had greater likelihood of waiting until a condom was available in the Low Pregnancy Risk condition when compared to reproductive-aged men $(p=.014)$. No significant differences were observed for the other post-hoc comparisons.

A Mann-Whitney was run to test median AUC differences between reproductive-aged men and reproductive-aged women (i.e., excluding women that could not become pregnant at the time of the survey) on the six Sexual Discounting conditions. No significant differences were observed. See Table 12 for the full list of comparisons. 
Relationship status. To compare participants based on current relationship status, a composite variable was created. Participants who responded "married" and "separated" were combined to create a variable representing participants that are "in a relationship" $(n=31)$. Participants who responded, "never married”, “divorced”, "living with a partner", and "widowed" were combined to create a variable representing participants that are "not in a relationship" ( $n=82)$. A Mann-Whitney was run to test median AUC differences between participants "in a relationship" and "not in a relationship" on the six Sexual Discounting conditions. No significant differences were observed. See Table 13 for the full list of comparisons.

\section{Discussion}

Delay discounting is an important marker of domain-specific impulsive decision-making (Dalley et al., 2011). Various delay-discounting tasks can be used to assess the role that delay has on our preference between smaller-sooner or larger-later rewards (Paglieri, 2013). Previous research has shown that the sexual discounting task was domain and condition dependent. However, this prior research was limited by methodological concerns and a narrow scope that frequently did not include individual difference variables. There are several points of intersection between the pressing, wide-spread opioid epidemic and sexual risk behaviors including neonatal abstinence (Patrick et al., 2012), unintended pregnancies (Heil et al., 2011), and limited use of birth control (Terplan et al., 2015). Therefore, broadening our understanding of sexual decisionmaking through the inclusion of individual difference variables can inform the development of high efficacy sexual health interventions in adults with substance use disorder.

The current study is the first to include future time perspective, condom attitudes, and sexual arousal to broaden our understanding of the role of these individual difference variables in 
the likelihood of waiting for an available condom as assessed through the Sexual Discounting Task. The current study is also the first to include a pregnancy risk condition. This new pregnancy risk condition extends the focus of previous research that compared sexual attraction and STD risk conditions. Pregnancy risk is especially important in the Opioid Use Disorder patient populations because roughly $90 \%$ of women who present as pregnant in-treatment are experiencing an unintended pregnancy (Heil et al., 2011). The introduction of the new pregnancy risk condition necessitates a systematic approach. First, between-condition differences in delay discounting were tested. Second, within-condition differences in delay discounting were tested. After establishing the conditions are distinct and discounting occurs in expected direction and magnitude, the role of individual difference variables was tested. This study addressed three research questions: RQ1, are there domain differences in delay discounting; RQ2, does reward magnitude influence preference for monetary and sexual discounting; RQ3, are differences in sexual discounting associated with individual differences.

\section{Domain Differences between Delay Discounting AUCs}

The first goal of the present study was to characterize between-domain differences (i.e., money vs. sex). Previous research has established that the Sexual Discounting Task AUC was not correlated with the Monetary Delay-Discounting Task AUC (Johnson \& Bruner, 2012, 2013; M. W. Johnson et al., 2015). However, these studies did not report directly testing AUC differences between each domain (i.e., monetary vs. sex). Although a lack of correlation could indicate differences in AUC between domains, a direct test of differences is preferred. Therefore, to directly test differences in AUC between the Monetary-Delay Discounting Task and the Sexual Delay Discounting Task a non-parametric, Wilcoxon Signed-ranks Test was used to examine domain differences. Based on this series of analyses using the $\$ 10$ and $\$ 100$ Monetary 
AUC, significant differences in AUC were observed. Lower AUCs were observed in the \$10 Monetary condition. This suggest a greater preference for smaller-sooner rewards in the $\$ 10$ Monetary condition than immediate condom-less sexual intercourse for the Low Attraction, High STD Risk, High Pregnancy Risk, and Low Pregnancy Risk conditions. This suggests that in conditions with low attraction and high risk or conditions where the risk might be more salient like the potential for pregnancy, there was a greater likelihood of waiting until a condom was available or waiting for the delayed reward.

An important distinction was observed between the \$10 and \$100 Monetary conditions when examining differences with the High Attraction condition. The \$10 Monetary AUC was not significantly different from High Attraction AUC; however, a difference was observed between \$100 Monetary AUC and High Attraction AUC. The \$100 Monetary AUC was higher than the High Attraction AUC. This suggests that in the High Attraction condition there was less likelihood of waiting until a condom was available or a greater preference for the immediately available reward compared to the $\$ 100$ Monetary condition. Said another way, a highly attractive, willing, and available sexual partner shifts preference towards immediate rewards more than $\$ 100$. Compared to the $\$ 10$ Monetary condition, the $\$ 100$ Monetary condition had similar results for the comparisons between the $\$ 100$ Monetary AUC and the High STD Risk AUC and High Pregnancy Risk AUC. A lower AUC was observed for the \$100 Monetary condition. This suggest a greater preference for smaller-sooner rewards in the $\$ 100$ Monetary condition than immediate condom-less sexual intercourse for the High STD Risk condition and High Pregnancy Risk condition. This indicates in conditions with high risk, there was a greater likelihood of waiting until a condom was available. 
Taken together, these comparisons between domains indicate that there are important domain differences in reward evaluation between monetary and sex rewards. Therefore, Monetary Delay-Discounting Tasks should not be used instead of Sexual-Discounting Tasks if the experiment is concerned with risky sexual behavior. Additionally, money has been shown to be a more powerful reward when compared to high-risk sexual situations as reflected in lower AUC for the \$10 and \$100 Monetary conditions when compared to High STD and High Pregnancy Risk conditions. This could inform future intervention testing and development. Contingency management, a reward-based approach to behavior treatment for addiction uses rewards to increase the frequency of a desired therapeutic behavior (e.g., Petry, 2011). The contingency management relies on rewards with high reward value to increase the behavior. In this context, it appears that even small amounts of money, $\$ 10$, have a high reward value compared to condoms in risky sexual conditions. However, condoms in low risk sexual situations (i.e., low STD risk) were not significantly different and this could suggest that condoms or other sexual products could serve as rewards in a contingency management framework. Future research could investigate using high quality sexual products as part of a contingency management framework. Use of reproductive health methods is generally low in this population (Terplan et al., 2015) and approaches that increase treatment compliance in addition to reproductive health goals could create great value for a patient and clinic.

It is important to note that all four of the studies (Herrmann et al., 2014; Johnson \& Bruner, 2012, 2013; M. W. Johnson et al., 2015) that tested domain differences (i.e., money vs. sex) used longer, less ecologically relevant delays (i.e., 1 hour, 3 hours, 6 hours, 1 day, 1 week, 1 month, and 3 months) instead of shorter, more ecologically relevant delays (i.e., 0 minute, 2 minute, 5 minute, 15 minute, 30 minute, 1 hour, 3 hours, 6 hours) like those used in the present 
study. Ecologically relevant delays are more than a methodological curiosity. Assessing "heat of the moment" sexual decision-making where the sexual partner is immediately available and willing can be more accurately captured by using shorter delays because this context is highly salient (Johnson et al., 2017; Stoltman et al., 2015). Additionally, differences in likelihood of waiting until a condom was available (i.e., AUC) were different between conditions with these shorter delays, suggesting that ecologically relevant delays provide a valuable approach to assess sexual discounting in a hypothetical context.

\section{Condition Differences between Delay Discounting AUCs}

After establishing between domain differences, the present study also explored domain differences within the various Sexual Discounting Tasks. The High Attraction condition AUC was significantly lower than all other Sexual Discounting Task AUCs except for the Low STD Risk condition. Similarly, the Low STD Risk condition AUC was significantly lower than all other Sexual Discounting Task condition AUCs except for the High Attraction condition. This indicates that participants were similarly less likely to wait until a delayed condom was available in both the High Attraction and Low STD Risk condition. These two conditions had the highest reward value out of the six Sexual-Discounting Task conditions assessed. While High Attraction and Low STD Risk could be considered lower risk, they are not no risk sexual conditions. Future interventions can prioritize these two conditions (i.e., High Attraction and Low STD Risk) as the greatest likelihood to not wait for a condom. This might suggest that participants who have less likelihood to wait for delayed condoms in these conditions could benefit from enhanced contraceptive counseling that prioritizes long-acting reversible contraceptive use in addition to condom use. The best form of contraception use is dual use or "condoms plus" because this is the only approach to reduce both unintended pregnancies and STD risk (Cates \& Steiner, 2002). 
It is important to note that the Low Pregnancy Risk AUC was significantly higher than both High Attraction AUC and Low STD Risk AUC; although, the Low Pregnancy Risk condition could be considered conceptually similar to the Low STD Risk condition. Further support for this difference in STD risk and pregnancy risk comes from the direct comparison of STD Risk and Pregnancy Risk. There was no significant difference between the High STD Risk and High Pregnancy Risk conditions suggest that participants evaluated them similarly. Taken together, these findings involving STD risk and pregnancy risk suggest that pregnancy risk could be more salient than STD risk. When participants were prompted to imagine a scenario with an immediately available and willing partner that could get pregnant, even a low risk, preferences shifted toward a greater likelihood to wait until a condom is available.

Future studies could include more information related to history with STD treatment and familiarity with STDs. For example, it is not known from the current study what STD they were recalling during the task. Using a specific STD might be preferable in the future. For example, they may have been considering an STD that is readily treatable like gonorrhea rather than the treatment resistant-gonorrhea that is growing in prevalence (Weston, Workowski, Torrone, Weinstock, \& Stenger, 2018). It is possible that a generic STD risk was not as salient as a specific pregnancy risk that entails a life-time of commitment. Future studies could also use more graphic descriptions of the STD to determine whether this influences likelihood of waiting for a condom. It is also worth noting that this is the first time the pregnancy risk condition has been used; therefore, further research into the reliability and replicability of this finding is important.

\section{Reward Magnitude Differences}


Given that there are domain differences between monetary and sexual delay discounting, it is important to further understand if there are differences in reward magnitude within each condition. Magnitude differences can indicate that likelihood of waiting for a condom is context specific (Johnson \& Bruner, 2012). Establishing within condition differences are especially important considering that this study included the novel pregnancy risk condition. Magnitude differences were observed within each condition for AUC. This suggests that participants were sensitive to condition differences and responded in ways that were consistent with the task. For example, in the High Attraction condition, participants had a lower likelihood of waiting until a condom was available compared to the Low Attraction condition. This which suggests that when the sexual partner is perceived to be highly attractive, participants were less likely to wait for a delayed condom than when the hypothetical partner was regarded to have low levels of attraction. Similarly, both risk conditions, STD and pregnancy, were likelihood of waiting for a condom was characteristic of risk. That is, high risk conditions had a greater likelihood of waiting until a condom was available than the low risk conditions. These results are consistent with the previous Sexual Discounting Task studies (Herrmann et al., 2014; Johnson \& Bruner, 2012, 2013; Johnson et al., 2017; M. W. Johnson et al., 2015) and suggest that the new pregnancy risk condition is consistent with what would be expected. These results also suggest that participants, even with a hypothetical laboratory task, had some insight into their behavior when evaluating risky sexual situations. It is possible that in a real-world evaluation, preferences would shift more than the laboratory-based task, however, the present study suggests that there are differences in how risky sexual behavior may drive decision-making. Future studies could evaluate whether these conditions could be used to develop a screener for adults to determine if 
adults with less likelihood of waiting for a delayed condom in high risk conditions need higher levels of care or more targeted interventions.

\section{Individual Differences and Sexual Discounting}

Previous research in individual differences and sexual discounting has been primarily centered on gender differences whereby women had a greater likelihood of waiting until a condom was available than men (Jarmolowicz et al., 2014; Johnson \& Bruner, 2013; Lawyer \& Schoepflin, 2013). Gender differences could inform how condom-based birth control information is provided. The lack of significant differences for our Sexual-Discounting Task conditions by gender contradict previous findings. However, this is the first study to test gender differences with hypothetical, ecologically relevant delays. These findings suggest that, when using shorter delays, women and men have similar likelihood of waiting until a condom is available. Future research could better evaluate gender through questionnaires about endorsement with gender roles and sexual decision making based on gender roles to further understand if there are gender differences in sexual discounting.

Previous research suggests that Future Time Perspective is related to condom use in that male sex workers with higher future orientation had higher condom use with regular partners (Sosa-Rubí et al., 2018). The current study includes various elements of future time orientation to better understand the association between future time perspective and sexual delay discounting. Future as Open was not significantly correlated with any Sexual Discounting Task AUCs. Future as Limited was significantly negatively correlated with the Low Pregnancy Risk AUC. This suggests that participants with less likelihood of waiting until a condom is available (i.e., low AUC) had higher endorsement of the future as limited. Similarly, Future as Ambiguous was negatively correlated with the Low Attraction AUC and the Low Pregnancy Risk AUC. This 
suggests that participants with less likelihood of waiting until a condom is available (i.e., low AUC) had higher endorsement of the future as ambiguous. Future research could continue to evaluate the role of future time perspective and sexual discounting in diverse samples. This is the first study to explicitly include future time perspective and sexual discounting. Future time perspective can be modified through an intervention called Episodic Future Thinking (Atance \& O'Neill, 2001; Kaplan, Reed, \& Jarmolowicz, 2016). Episodic Future Thinking works through shifting time perspective or expanding an adults time horizon. For example, in adults with high future as ambiguous, an Episodic Future Thinking intervention could shift their future orientation to a more open and therefore reduce the risks associated with less likelihood of waiting for a condom in a low attraction or low pregnancy risk scenario.

Condom attitudes have not been directly assessed in previous Sexual Discounting Task research; however, there is reason to believe that condom attitudes can influence condom use (Hood \& Shook, 2013). This study supports the notion that various elements of condom attitudes have different associations with sexual delay discounting. For example, higher endorsement of condoms as pleasurable was associated with greater likelihood of waiting until a delayed condom was available in the High Attraction, Low STD Risk, and Low Pregnancy Risk conditions. Null findings for condom pleasure and Low Attraction, High STD Risk, and High Pregnancy Risk suggest that condom pleasure is not enough to impact willingness to wait for delayed condoms when there is high risk. Additionally, the median for High STD Risk and High Pregnancy Risk was effected by a ceiling effect. This might be due to the shorter, more ecologically valid delays or components unique to these conditions. Participants with higher pleasure from condom use endorsed an overall greater likelihood of waiting until a condom was available. Therefore, improving condom pleasure in future interventions through designs that increase the availability 
of high-quality condoms could change these preferences for those who find condoms to be less pleasurable. Additionally, future interventions could improve the dissemination of information around proper condom use.

Condom Stigma was positively associated with the High STD Risk condition, the High Pregnancy Risk condition, and the Low Pregnancy Risk condition. This suggests that participants who perceived condom use to have high stigma had higher AUC or less preference for immediate condom-less sexual intercourse. Participants with less stigma surrounding condom use were more likely to wait for delayed condoms. Similarly, MCAS Embarrassment About Negotiation and Use of Condoms of was positively associated with both the Low Attraction and the High Pregnancy Risk conditions. Participants with lower likelihood of waiting until a delayed condom was available had more agreement that condom use and negotiations surrounding condom use were embarrassing. These findings suggest that interventions targeting Condom Stigma and techniques to address Embarrassment About Negotiation and Use could contribute to a shift in condom use preferences in high risk situations. Sexual arousal was negatively associated with High Pregnancy Risk which suggests that in participants who had higher sexual arousal, there was less likelihood of waiting until a delayed condom was available in the High Pregnancy Risk condition. However, arousal levels were fairly low in the sample $(M=46.8 \%)$. Future research could better elicit sexual arousal using techniques discussed by Ariely and Loewenstein (2006) such as engaging in manual self-stimulation while watching pornographic videos and evaluating sexual risk behaviors. Mindfulness interventions could be used to help adults at higher risk for engaging in risky-sexual behavior when aroused to reorient and reevaluate their decision. 
Age was positively associated with a greater likelihood of waiting until a condom was available in the High Attraction, Low STD Risk, and High Pregnancy Risk conditions. These positive associations between with the High Attraction and Low STD Risk conditions are noteworthy because participants answering questions during these two conditions generally had the lowest preference for a delayed condom. Additionally, the High Pregnancy Risk condition was primarily associated with greater preference for delayed condoms, so it is noteworthy that older participants were potentially driving this effect. It is noteworthy that due to the nonparametric nature of the data, more advance analysis such as a mediation or moderation are not recommended. Future research could include larger, more diverse samples to determine whether the response restriction was due to unique sample characteristics or is inherent when using the shorter, more ecologically relevant delays.

\section{Limitations}

Although there is evidence from the current data that hypothetical tasks can elucidate difference in choice for delayed rewards as shown through both the between and within condition comparisons, it is unclear from this study how participants would behave in a realworld situation. Rates of non-systematic data in our sample were high (68\%) compared to previous studies that ranged from 6\% (Johnson \& Bruner, 2013) to 12.5\% (Johnson \& Bruner, 2012). A roughly equivalent sample of women in-treatment for Opioid Use Disorder had a 5\% rate of non-systematic data (Herrmann et al., 2014). To reduce the amount of non-systematic data, experimental procedures could be improved in future Sexual Discounting Task administration. Only 27 participants had complete data across all discounting tasks. Future tasks could include terminating the task or interrupt the task to allow for questions if non-systematic data is detected in real-time. Adjusting delay tasks have been developed for Monetary Delay- 
Discounting Tasks (Koffarnus \& Bickel, 2014); however, these tasks have not yet been developed for the Sexual Discounting Task. An adjusting delay task uses a dichotomous choice instead of adjusting amounts that is quicker and ensures systematic data responding. Although this approach cannot eliminate random responding and, it should be evaluated further in future research studies. In this nascent stage of testing the Sexual Discounting Task, it remains important to validate old procedures while balancing the development of tasks that can more accurately capture responding. Issues with tablet sensitivity could also contribute to the high-rate of non-systematic responding in our sample. Future research could include comparisons with paper-and-pencil task administration to isolate whether the technology caused unnecessary complications in task administration. Ultimately, participant burden could have contributed to non-systematic responding. The discounting wave (Wave 2) was only 15 minutes, however, it was completed after participants completed group therapy and medication management (1.5 hours of doctor's appointments). An unknown number also had other appointments that day and external contingencies (e.g., needing to share rides, child care, long travel times) that could have contributed to rushing through some portions of the task. Lastly, using eight discounting tasks in a row can appear redundant because the differences between tasks was subtle. Future research should explore these methods and others to reduce participant burden.

Generalizability of this study is difficult to determine because the sample did not include other in-treatment populations, other conditions associated with impulsive choice responding, or healthy controls. Therefore, to better understand hypothetical sexual delay discounting, a broader survey of patient populations and age groups are needed. The sample derived from the clinic population was a convenience sample and may not represent response patterns for patients in treatment for opioid use disorder. The clinic enrolls roughly 600 patients, and this sample 
included 113. The nature of the questions (sexual and personal) was disclosed during recruiting and may have contributed to response biases or self-section biases. Due to time limitations surrounding recruiting, not all patients at the clinic were approached for the study. Most notably, patients further along in their recovery interact less frequently with the COAT clinic and therefore were under sampled. Not all groups and patients in the clinic Future research could work to include this information into screening material at the clinic. Additionally, overall condom use was low in this sample. This is reflective of patients in-treatment opioid use disorder (Terplan et al., 2015), but may suggest that questions about future condom use are less relevant outside of the context of an intervention to increase condom use. This study includes multiple comparisons that can contribute to inflated Type 1 error rates. Therefore, results should be interpreted with caution prior to replication in other larger, more diverse samples. Lastly, due to the data presented in the published manuscript, it is unclear how participants in the present study compared to Johnson et al. (2017), the only other study to use these same delays.

Despite several weaknesses, this study has unique strengths that can advance the study of sexual discounting and our understanding of sexual decision-making. Methodological advancements from this study include the use of ecologically relevant delays (e.g., minutes, hours) and a new attractiveness rating system. The majority of earlier sexual discounting work has used longer delays (e.g., weeks, months) to compare with monetary delay-discounting tasks. This study used ecologically relevant delays (e.g., minutes, hours) for both monetary and sexual discounting tasks. In contrast to the work by Johnson et al. (2017) that used shorter, ecologically relevant delays for the Low STD Risk condition, the present study assessed sexual discounting across all four of the original conditions (i.e., High/Low Attractiveness, High/Low STD Risk) in addition to the new High/Low Pregnancy Risk condition. This study is the first time a 
streamlined version of the sexual discounting task attractiveness rating is a significant advancement on the previous rating system. Previous versions of the Sexual Discounting Task involved the participant rating 60 photos to identify who they thought was the most/least attractive and high/low STD risk (Johnson \& Bruner, 2012). Determining the efficacy of this new rating system can lead to increased use of the sexual discounting task as a screening tool in clinical settings. While expediency is gained with this new approach to attractiveness rating, this hypothetical approach adds additional confounds. For example, a participant might imagine their partner or spouse that is either too old to become pregnant or not at risk for STDs. Future studies that use this approach can include more explicit instructions to reduce this potential issue.

\section{Future Directions and Conclusions}

Future research can build upon the present study. Little is known about stability in sexual-delay discounting past one-week (Johnson \& Bruner, 2013). For example, future research could investigate change and stability of sexual delay discounting throughout the duration of treatment for opioid use disorder and that could be used to identify patients at high risk for risky sexual intercourse. Additionally, future research can continue to use ecologically relevant delays to better proxy real-world sexual decision-making. Lastly, this task used hypothetical scenarios to elicit sexual arousal instead of the lengthy picture rating task. Future research could validate this approach to advance its use in other studies.

When considering the role of sexual arousal and Sexual Discounting Task AUC, the role of an intervention is complicated (Ariely \& Loewenstein, 2006). For example, because higher sexual arousal was associated with lower preference for hypothetical condom use during the High Pregnancy Risk condition, interventions could bolster mindfulness during sexually arousing situations. However, overall significant differences in Sexual Discounting AUC might 
be tempered by sexual arousal. The "hot-cold" empathy gap theorizes that during emotionally salient decisions, the preference could be different. Therefore, the ability to reduce the effect of sexual arousal on risky sexual decision-making hinges on the fact that individuals do not predict how they would respond in emotionally salient contexts when they are asked in times with less emotional stimuli. This suggests that pregnancy risk is more salient than STD risk and thinking of pregnancy risk can shift preference for delays.

This study advances the field in four important ways. First, this study has found condition differences between monetary and sexual discounting. Second, within condition differences in magnitude were observed for all the tasks including the new pregnancy risk task. Third, individual difference variables suggest that gender might differ less in sexual discounting when considering ecologically relevant delays; however, time perspective and condom attitudes were associated with specific sexual discounting conditions. Fourth, the present study may offer two methodological advancements: first, the inclusion of a new, hypothetical exercise for imaging a sexual partner rather than the traditional picture rating system and, second, the use of ecologically relevant delays. Still, the task is hypothetical and might not proxy real-world highly emotionally salient decisions. 


\section{References}

Adinoff, B., Rilling, L. M., Williams, M. J., Schreffler, E., Schepis, T. S., Rosvall, T., \& Rao, U. (2007). Impulsivity, neural deficits, and the addictions: The "oops" factor in relapse. Journal of Addictive Diseases, 26 Suppl 1(0 1), 25-39. doi:10.1300/J069v26S01_04

Argyriou, E., Um, M., Carron, C., \& Cyders, M. A. (2018). Age and impulsive behavior in drug addiction: A review of past research and future directions. Pharmacology Biochemistry and Behavior, 164, 106-117. doi:10.1016/j.pbb.2017.07.013

Ariely, D., \& Loewenstein, G. (2006). The heat of the moment: The effect of sexual arousal on sexual decision making. Journal of Behavioral Decision Making, 19(2), 87-98. Retrieved from https://onlinelibrary-wiley-com.proxy.lib.wayne.edu/doi/pdf/10.1002/bdm.501

Atance, C. M., \& O'Neill, D. K. (2001). Episodic future thinking. Trends in Cognitive Sciences, $5(12), 533-539$.

Baltes, P. B. (1987). Theoretical propositions of life-span developmental psychology: On the dynamics between growth and decline. Developmental Psychology, 23(5), 611.

Berry, M. S., \& Johnson, M. W. (2018). Does being drunk or high cause HIV sexual risk behavior? A systematic review of drug administration studies. Pharmacology Biochemistry and Behavior, 164, 125-138. doi:10.1016/j.pbb.2017.08.009

Bickel, W. K., \& Mueller, E. T. (2009). Toward the study of trans-disease processes: A novel approach with special reference to the study of co-morbidity. Journal of Dual Diagnosis, 5(2), 131-138. doi:10.1080/15504260902869147

Broos, N., Schmaal, L., Wiskerke, J., Kostelijk, L., Lam, T., Stoop, N., . . Goudriaan, A. E. (2012). The relationship between impulsive choice and impulsive action: A cross-species translational study. PloS One, 7(5), e36781. doi:10.1371/journal.pone.0036781 
Brothers, A., Chui, H., \& Diehl, M. (2014). Measuring future time perspective across adulthood: Development and evaluation of a brief multidimensional questionnaire. Gerontologist, 54(6), 1075-1088. doi:10.1093/geront/gnu076

Carstensen, L. L. (1991). Selectivity theory: Social activity in life-span context. Annual Review of Gerontology and Geriatrics, 11, 195-217.

Carstensen, L. L., Isaacowitz, D. M., \& Charles, S. T. (1999). Taking time seriously. A theory of socioemotional selectivity. American Psychologist, 54(3), 165-181. Retrieved from http://www.ncbi.nlm.nih.gov/pubmed/10199217

Cates, W., Jr., \& Steiner, M. J. (2002). Dual protection against unintended pregnancy and sexually transmitted infections: What is the best contraceptive approach? Sexually Transmitted Disease, 29(3), 168-174.

Copen, C. E. (2017). Condom Use During Sexual Intercourse Among Women and Men Aged 15-44 in the United States: 2011-2015 National Survey of Family Growth. National Health Statistics Reports(105), 1-18.

Cosenza, M., Griffiths, M. D., Nigro, G., \& Ciccarelli, M. (2017). Risk-taking, delay discounting, and time perspective in adolescent gamblers: An experimental study. Journal of Gambling Studies, 33(2), 383-395. doi:10.1007/s10899-016-9623-9

Dalley, J. W., Everitt, B. J., \& Robbins, T. W. (2011). Impulsivity, compulsivity, and top-down cognitive control. Neuron, 69(4), 680-694. doi:10.1016/j.neuron.2011.01.020

Dougherty, D. M., Lake, S. L., Mathias, C. W., Ryan, S. R., Bray, B. C., Charles, N. E., \& Acheson, A. (2015). Behavioral impulsivity and risk-taking trajectories across early adolescence in youths with and without family histories of alcohol and other drug use 
disorders. Alcoholism, Clinical and Experimental Research, 39(8), 1501-1509. doi:10.1111/acer.12787

Field, A. (2013). Discovering statistics using IBM SPSS statistics: Sage.

Field, M., Santarcangelo, M., Sumnall, H., Goudie, A., \& Cole, J. (2006). Delay discounting and the behavioural economics of cigarette purchases in smokers: The effects of nicotine deprivation. Psychopharmacology, 186(2), 255-263. doi:10.1007/s00213-006-0385-4

Giordano, L. A., Bickel, W. K., Loewenstein, G., Jacobs, E. A., Marsch, L., \& Badger, G. J. (2002). Mild opioid deprivation increases the degree that opioid-dependent outpatients discount delayed heroin and money. Psychopharmacology, 163(2), 174-182. doi:10.1007/s00213-002-1159-2

Gollner, L. M., Ballhausen, N., Kliegel, M., \& Forstmeier, S. (2017). Delay of gratification, delay discounting and their associations with age, episodic future thinking, and future time perspective. Frontiers in Psychology, 8, 2304. doi:10.3389/fpsyg.2017.02304

Green, L., \& Myerson, J. (2004). A discounting framework for choice with delayed and probabilistic rewards. Psychological Bulletin, 130(5), 769-792. doi:10.1037/00332909.130.5.769

Green, L., Myerson, J., Oliveira, L., \& Chang, S. E. (2013). Delay discounting of monetary rewards over a wide range of amounts. The Journal of The Experimental Analysis of Behavior, 100(3), 269-281. doi:10.1002/jeab.45

Green, L., Myerson, J., Oliveira, L., \& Chang, S. E. (2014). Discounting of delayed and probabilistic losses over a wide range of amounts. The Journal of The Experimental Analysis of Behavior, 101(2), 186-200. Retrieved from https://www.ncbi.nlm.nih.gov/pubmed/24745086 
Han, B., Compton, W. M., Blanco, C., \& Jones, C. M. (2018). Prescription opioid use, misuse, and use disorders in US adults. Annals of Internal Medicine, 168(5), 383-384. Retrieved from https://annals.org/aim/article-abstract/2674096/prescription-opioid-use-misuse-usedisorders-u-s-adults?doi=10.7326\%2fL17-0700

Hedegaard, H., Bastian, B. A., Trinidad, J. P., Spencer, M., \& Warner, M. (2018). Drugs most frequently involved in drug overdose deaths: United States, 2011-2016. MMWR CDC Surveillance Summaries.

Heil, S. H., Jones, H. E., Arria, A., Kaltenbach, K., Coyle, M., Fischer, G., . . Martin, P. R. (2011). Unintended pregnancy in opioid-abusing women. Journal of Substance Abuse Treatment, 40(2), 199-202. doi:10.1016/j.jsat.2010.08.011

Helweg-Larsen, M., \& Collins, B. E. (1994). The UCLA Multidimensional Condom Attitudes Scale: Documenting the complex determinants of condom use in college students. Health Psychology, 13(3), 224-237. Retrieved from https://www.ncbi.nlm.nih.gov/pubmed/8055858

Herrmann, E. S., Hand, D. J., Johnson, M. W., Badger, G. J., \& Heil, S. H. (2014). Examining delay discounting of condom-protected sex among opioid-dependent women and nondrug-using control women. Drug and Alcohol Dependence, 144, 53-60. doi:10.1016/j.drugalcdep.2014.07.026

Hood, K. B., \& Shook, N. J. (2013). Conceptualizing women's attitudes toward condom use with the tripartite model. Women \& Health, 53(4), 349-368. doi:10.1080/03630242.2013.788610 
Jarmolowicz, D. P., Bickel, W. K., \& Gatchalian, K. M. (2013). Alcohol-dependent individuals discount sex at higher rates than controls. Drug and Alcohol Dependence, 131(3), 320323. doi:https://doi.org/10.1016/j.drugalcdep.2012.12.014

Jarmolowicz, D. P., Landes, R. D., Christensen, D. R., Jones, B. A., Jackson, L., Yi, R., \& Bickel, W. K. (2014). Discounting of money and sex: Effects of commodity and temporal position in stimulant-dependent men and women. Addictive Behaviors, 39(11), 16521657. doi:https://doi.org/10.1016/j.addbeh.2014.04.026

Johnson, M. W., \& Bickel, W. K. (2008). An algorithm for identifying nonsystematic delaydiscounting data. Experimental and Clinical Psychopharmacology, 16(3), 264-274. doi:10.1037/1064-1297.16.3.264

Johnson, M. W., \& Bruner, N. R. (2012). The Sexual Discounting Task: HIV risk behavior and the discounting of delayed sexual rewards in cocaine dependence. Drug and Alcohol Dependence, 123(1-3), 15-21. doi:10.1016/j.drugalcdep.2011.09.032

Johnson, M. W., \& Bruner, N. R. (2013). Test-retest reliability and gender differences in the sexual discounting task among cocaine-dependent individuals. Experimental and Clinical Psychopharmacology, 21(4), 277-286. doi:10.1037/a0033071

Johnson, M. W., Herrmann, E. S., Sweeney, M. M., LeComte, R. S., \& Johnson, P. S. (2017). Cocaine administration dose-dependently increases sexual desire and decreases condom use likelihood: The role of delay and probability discounting in connecting cocaine with HIV. Psychopharmacology, 234(4), 599-612. doi:10.1007/s00213-016-4493-5

Johnson, M. W., Johnson, P. S., Herrmann, E. S., \& Sweeney, M. M. (2015). Delay and probability discounting of sexual and monetary outcomes in individuals with cocaine use 
disorders and matched controls. PloS One, 10(5), e0128641-e0128641.

doi:10.1371/journal.pone.0128641

Johnson, P. S., Herrmann, E. S., \& Johnson, M. W. (2015). Opportunity costs of reward delays and the discounting of hypothetical money and cigarettes. Journal of the Experimental Analysis of Behavior, 103(1), 87-107. Retrieved from https://www.ncbi.nlm.nih.gov/pmc/articles/PMC4428151/pdf/nihms685135.pdf

Kaplan, B. A., Reed, D. D., \& Jarmolowicz, D. P. (2016). Effects of episodic future thinking on discounting: Personalized age-progressed pictures improve risky long-term health decisions. Journal of Applied Behavior Analysis, 49(1), 148-169. doi:10.1002/jaba.277

Kirby, K. N., \& Marakovic, N. N. (1996). Delay-discounting probabilistic rewards: Rates decrease as amounts increase. Psychonomic Bulletin \& Review, 3(1), 100-104. doi:10.3758/bf03210748

Koffarnus, M. N., \& Bickel, W. K. (2014). A 5-trial adjusting delay discounting task: Accurate discount rates in less than one minute. Experimental and Clinical Psychopharmacology, 22(3), 222-228. doi:10.1037/a0035973

Lawyer, S. R., \& Schoepflin, F. J. (2013). Predicting domain-specific outcomes using delay and probability discounting for sexual versus monetary outcomes. Behavioural Processes, 96, 71-78. doi:https://doi.org/10.1016/j.beproc.2013.03.001

Lawyer, S. R., Williams, S. A., Prihodova, T., Rollins, J. D., \& Lester, A. C. (2010). Probability and delay discounting of hypothetical sexual outcomes. Behavioural Processes, 84(3), 687-692. doi:https://doi.org/10.1016/j.beproc.2010.04.002 
Linley, L., Johnson, A. S., Song, R., Wu, B., Hu, S., Singh, S., . . Hernandez, A. (2018). Estimated HIV incidence and prevalence in the United States 2010-2015. MMWR CDC Surveillance Summaries.

Liu, Y., \& De, A. (2015). Multiple imputation by fully conditional specification for dealing with missing data in a large epidemiologic study. International Journal of Statistics in Medical Research, 4(3), 287-295. doi:10.6000/1929-6029.2015.04.03.7

Mack, K. A., Frazier, L., Terplan, M., Iskander, J. K., Thorpe, P., \& Laird, S. (2017). Addressing the unique challenges of opioid use disorder in women. CDC Grand Rounds.

Mazur, J. E. (1987). An adjusting procedure for studying delayed reinforcement. In The effect of delay and of intervening events on reinforcement value. (pp. 55-73). Hillsdale, NJ, US: Lawrence Erlbaum Associates, Inc.

Mitchell, S. H. (2004). Effects of short-term nicotine deprivation on decision-making: Delay, uncertainty and effort discounting. Nicotine \& Tobacco Research, 6(5), 819-828. Retrieved from http://www.ncbi.nlm.nih.gov/pubmed/15700917

Moody, L., Franck, C., Hatz, L., \& Bickel, W. K. (2016). Impulsivity and polysubstance use: A systematic comparison of delay discounting in mono-, dual-, and trisubstance use. Experimental and Clinical Psychopharmacology, 24(1), 30-37. doi:10.1037/pha0000059

Murthy, V. H. (2016). Surgeon general's report on alcohol, drugs, and health. JAMA. doi:10.1001/jama.2016.18215

Myerson, J., Green, L., \& Warusawitharana, M. (2001). Area under the curve as a measure of discounting. Journal of the Experimental Analysis of Behavior, 76(2), 235-243. doi:10.1901/jeab.2001.76-235 
Obeid, J. S., McGraw, C. A., Minor, B. L., Conde, J. G., Pawluk, R., Lin, M., . . Harris, P. A. (2013). Procurement of shared data instruments for Research Electronic Data Capture (REDCap). Journal of Biomedical Informatics, 46(2), 259-265.

doi:https://doi.org/10.1016/j.jbi.2012.10.006

Odum, A. L. (2011a). Delay discounting: I'm a k, you're a k. Journal of the Experimental Analysis of Behavior, 96(3), 427-439. doi:10.1901/jeab.2011.96-423

Odum, A. L. (2011b). Delay discounting: Trait variable? Behavioural Processes, 87(1), 1-9. doi:10.1016/j.beproc.2011.02.007

Odum, A. L., Baumann, A. A., \& Rimington, D. D. (2006). Discounting of delayed hypothetical money and food: Effects of amount. Behavioural Processes, 73(3), 278-284. doi:10.1016/j.beproc.2006.06.008

Odum, A. L., \& Rainaud, C. P. (2003). Discounting of delayed hypothetical money, alcohol, and food. Behavioural Processes, 64(3), 305-313. doi:https://doi.org/10.1016/S0376$\underline{6357(03) 00145-1}$

Paglieri, F. (2013). The costs of delay: Waiting versus postponing in intertemporal choice. Journal of the Experimental Analysis of Behavior, 99(3), 362-377. doi:10.1002/jeab.18

Patrick, S. W., Schumacher, R. E., Benneyworth, B. D., Krans, E. E., McAllister, J. M., \& Davis, M. M. (2012). Neonatal abstinence syndrome and associated health care expenditures: United States, 2000-2009. JAMA, 307(18), 1934-1940. doi:10.1001/jama.2012.3951

Pattij, T., \& De Vries, T. J. (2013). The role of impulsivity in relapse vulnerability. Current Opinion in Neurobiology, 23(4), 700-705. doi:10.1016/j.conb.2013.01.023

Petry, N. M. (2011). Contingency management: What it is and why psychiatrists should want to use it. The Psychiatrist, 35(5), 161-163. doi:10.1192/pb.bp.110.031831 
Reynolds, B., \& Schiffbauer, R. (2005). Delay of gratification and delay discounting: A unifying feedback model of delay-related impulsive behavior. The Psychological Record, 55(3), 439-460.

Rogers, R. D., Moeller, F. G., Swann, A. C., \& Clark, L. (2010). Recent research on impulsivity in individuals with drug use and mental health disorders: Implications for alcoholism. Alcoholism, Clinical and Experimental Research, 34(8), 1319-1333. doi:10.1111/j.15300277.2010.01216.x

Rosengard, C., Anderson, B. J., \& Stein, M. D. (2006). Correlates of condom use and reasons for condom non-use among drug users. American Journal of Drug and Alcohol Abuse, 32(4), 637-644. doi:10.1080/00952990600919047

Shaw, G. M., \& Hunter, E. (2012). HIV transmission. Cold Spring Harbor Perspectives in Medicine, 2(11). doi:10.1101/cshperspect.a006965

Shih, S. L., Kebodeaux, C. A., Secura, G. M., Allsworth, J. E., Madden, T., \& Peipert, J. F. (2011). Baseline correlates of inconsistent and incorrect condom use among sexually active women in the contraceptive CHOICE Project. Sexually Transmitted Diseases, 38(11), 1012-1019. doi:10.1097/OLQ.0b013e318225f8c3

Sosa-Rubí, S. G., Salinas-Rodríguez, A., Montoya-Rodríguez, A. A., \& Galárraga, O. (2018). The relationship between psychological temporal perspective and HIV/STI risk behaviors among male sex workers in Mexico City. Archives of Sexual Behavior, 47(5), 1551-1563. doi:10.1007/s10508-017-1123-1

Stein, J. S., Tegge, A. N., Turner, J. K., \& Bickel, W. K. (2018). Episodic future thinking reduces delay discounting and cigarette demand: An investigation of the good-subject effect. Journal of Behavioral Medicine, 41(2), 269-276. doi:10.1007/s10865-017-9908-1 
Stein, J. S., Wilson, A. G., Koffarnus, M. N., Daniel, T. O., Epstein, L. H., \& Bickel, W. K. (2016). Unstuck in time: Episodic future thinking reduces delay discounting and cigarette smoking. Psychopharmacology, 233(21), 3771-3778. doi:10.1007/s00213-016-4410-y

Stoltman, J. J. K., Woodcock, E. A., Lister, J. J., Lundahl, L. H., \& Greenwald, M. K. (2015). Heroin delay discounting: Modulation by pharmacological state, drug-use impulsivity, and intelligence. Experimental and Clinical Psychopharmacology, 23(6), 455-463. doi:10.1037/pha0000054

Terplan, M., Hand, D. J., Hutchinson, M., Salisbury-Afshar, E., \& Heil, S. H. (2015). Contraceptive use and method choice among women with opioid and other substance use disorders: A systematic review. Preventive Medicine, 80, 23-31. doi:10.1016/j.ypmed.2015.04.008

Tibbs, C. J. (1995). Methods of transmission of Hepatitis C. Journal of Viral Hepatitis, 2(3), 113-119. Retrieved from https://onlinelibrary.wiley.com/doi/abs/10.1111/j.13652893.1995.tb00016.x?sid=nlm\%3Apubmed

Verdejo-Garcia, A., Lawrence, A. J., \& Clark, L. (2008). Impulsivity as a vulnerability marker for substance-use disorders: Review of findings from high-risk research, problem gamblers and genetic association studies. Neuroscience and Biobehavioral Reviews, 32(4), 777-810. doi:10.1016/j.neubiorev.2007.11.003

Verges, A., Littlefield, A. K., Arriaza, T., \& Alvarado, M. E. (2019). Impulsivity facets and substance use initiation: A comparison of two models of impulsivity. Addictive Behaviors, 88, 61-66. doi:10.1016/j.addbeh.2018.08.018

Weatherly, J. N. (2014). On several factors that control rates of discounting. Behavioural Processes, 104, 84-90. doi:10.1016/j.beproc.2014.01.020 
Weston, E. J., Workowski, K., Torrone, E., Weinstock, H., \& Stenger, M. R. (2018). Adherence to CDC recommendations for the treatment of uncomplicated gonorrhea-STD Surveillance Network, United States, 2016. Morbidity and Mortality Weekly Report, 67(16), 473.

Woerner, J., Kopetz, C., Lechner, W. V., \& Lejuez, C. W. (2016). History of abuse and risky sex among substance users: The role of rejection sensitivity and the need to belong. Addictive Behaviors, 62, 73-78. doi:10.1016/j.addbeh.2016.06.006

Xu, S., Korczykowski, M., Zhu, S., \& Rao, H. (2013). Assessment of risk-taking and impulsive behaviors: A comparison between three tasks. Social Behavior and Personality, 41(3), 477-486. doi:10.2224/sbp.2013.41.3.477

Yoon, J. H., De La Garza, R., 2nd, Newton, T. F., Suchting, R., Weaver, M. T., Brown, G. S., . . . Haliwa, I. (2017). A comparison of Mazur's k and area under the curve for describing steep discounters. The Psychological Record, 67(3), 355-363. doi:10.1007/s40732-0170220-9

Yoon, J. H., \& Higgins, S. T. (2008). Turning k on its head: Comments on use of an ED50 in delay discounting research. Drug and Alcohol Dependence, 95(1-2), 169-172. doi:10.1016/j.drugalcdep.2007.12.011

Zheng, W., Nickasch, M., Lander, L., Wen, S., Xiao, M., Marshalek, P., . . Sullivan, C. (2017). Treatment outcome comparison between telepsychiatry and face-to-face buprenorphine medication-assisted treatment for opioid use disorder: A 2-year retrospective data analysis. Journal of Addiction Medicine, 11(2), 138-144.

doi:10.1097/ADM.0000000000000287 
Zibbell, J. E., Asher, A. K., Patel, R. C., Kupronis, B., Iqbal, K., Ward, J. W., \& Holtzman, D. (2018). Increases in acute hepatitis $\mathrm{C}$ virus infection related to a growing opioid epidemic and associated injection drug use, United States, 2004 to 2014. American Journal of Public Health, 108(2), 175-181. 
Table 1

Participant Characteristics

\begin{tabular}{|c|c|c|c|c|c|c|}
\hline & \multicolumn{2}{|c|}{$\begin{array}{c}\text { Analytic Sample } \\
(n=113)\end{array}$} & \multirow[b]{2}{*}{$\begin{array}{l}\text { Analytic } \\
\text { Sample } \\
(n=113)\end{array}$} & \multirow[b]{3}{*}{$F$} & \multirow[b]{3}{*}{$d f$} & \multirow[b]{3}{*}{$p$} \\
\hline & $\begin{array}{c}\text { Incomplete } \\
\text { Discounting } \\
\text { Data } \\
(n=86) \\
\end{array}$ & $\begin{array}{c}\text { Complete } \\
\text { Discounting } \\
\text { Data } \\
(n=27) \\
\end{array}$ & & & & \\
\hline Variable & & $M(S E)$ & & & & \\
\hline Age & $34.39(1.01)$ & $34.19(1.34)$ & $34.34(0.83)$ & 0.01 & 1,111 & .917 \\
\hline Education & $12.53(0.2)$ & $12.04(0.47)$ & $12.42(0.19)$ & 1.30 & 1,111 & .257 \\
\hline \multicolumn{7}{|l|}{ Future Time Perspective } \\
\hline Future as Open & $15.85(0.31)$ & $15.41(0.5)$ & $15.74(0.27)$ & 0.49 & 1,111 & .486 \\
\hline Future as Limited & $12.34(0.35)$ & $11.93(0.66)$ & $12.24(0.31)$ & 0.32 & 1,111 & .571 \\
\hline Future as Ambiguous & $11.9(0.40)$ & $11.48(0.64)$ & $11.8(0.34)$ & 0.27 & 1,111 & .605 \\
\hline \multicolumn{7}{|l|}{ MCAS } \\
\hline Pleasure & $3.15(0.12)$ & $3.54(0.21)$ & $3.25(0.10)$ & 2.54 & 1,111 & .114 \\
\hline Stigma & $5.53(0.14)$ & $6.03(0.17)$ & $5.65(0.11)$ & 3.63 & 1,111 & .060 \\
\hline Negotiation and Use & $5.31(0.14)$ & $5.79(0.19)$ & $5.42(0.12)$ & 3.16 & 1,111 & .078 \\
\hline \multirow[t]{2}{*}{ Sexual Arousal (\%) } & $46.78(3.89)$ & $40.85(6.11)$ & $45.36(3.30)$ & 0.59 & 1,111 & .446 \\
\hline & & $n(\%)$ & & $\chi^{2}$ & $d f$ & $\bar{p}$ \\
\hline Gender & & & & .34 & 1 & .560 \\
\hline Men & $24(27.9)$ & $6(22.2)$ & $30(26.5)$ & & & \\
\hline Women & $62(72.1)$ & $21(77.8)$ & $83(73.5)$ & & & \\
\hline Race & & & & 1.03 & 3 & .795 \\
\hline Non-White & $7(8.1)$ & $1(3.7)$ & $8(7.1)$ & & & \\
\hline White & 79 (91.9) & $26(96.3)$ & $105(92.9)$ & & & \\
\hline
\end{tabular}


Table 2

Descriptive Statistics for AUC Using Imputed

Data $(n=113)$

\begin{tabular}{lcc}
\hline & \multicolumn{2}{c}{ Raw AUC } \\
\hline Variable & $\begin{array}{c}\text { Median } \\
(\mathrm{IQR})\end{array}$ & $\begin{array}{c}\text { Mean } \\
(\mathrm{SE})\end{array}$ \\
\hline Attraction (High) & $.51(.86)$ & $.50(.04)$ \\
Attraction (Low) & $.73(.65)$ & $.66(.04)$ \\
STD Risk (High) & $1.00(.41)$ & $.80(.03)$ \\
STD Risk (Low) & $.58(.77)$ & $.56(.04)$ \\
Pregnancy Risk (High) & $1.00(.43)$ & $.77(.03)$ \\
Pregnancy Risk (Low) & $.73(.66)$ & $.65(.04)$ \\
Monetary (\$10) & $.33(.71)$ & $.46(.04)$ \\
Monetary (\$100) & $.63(.62)$ & $.60(.04)$ \\
\hline n. $b$. AUC = area under the curve; STD = \\
sexually transmitted infection; AUC is \\
calculated individually for each participant by \\
using the participant data at each time point. \\
AUC is on a 0 -1 scale. An AUC closer to 0 \\
indicates steeper discounting or lower \\
preference for delayed condom use.
\end{tabular}




\section{Table 3}

Dependent Samples Wilcoxon Signed-ranks Test Median

Differences Between and Within Conditions using Imputed AUC

Data $(n=113)$

\begin{tabular}{|c|c|c|c|}
\hline \multirow{2}{*}{ Variable } & & \multicolumn{2}{|c|}{ Raw $A U C$} \\
\hline & & $Z$ & $p$ \\
\hline \multirow[t]{7}{*}{ Attraction (High) } & Attraction (Low) & -4.03 & $<.001$ \\
\hline & STD Risk (High) & -5.51 & $<.001$ \\
\hline & STD Risk (Low) & -1.10 & .293 \\
\hline & Pregnancy Risk (High) & -6.44 & $<.001$ \\
\hline & Pregnancy Risk (Low) & -4.00 & $<.001$ \\
\hline & Monetary (\$10) & -0.54 & .605 \\
\hline & Monetary $(\$ 100)$ & -2.37 & .019 \\
\hline \multirow[t]{6}{*}{ Attraction (Low) } & STD Risk (High) & -3.05 & .009 \\
\hline & STD Risk (Low) & -2.68 & .010 \\
\hline & Pregnancy Risk (High) & -3.01 & .030 \\
\hline & Pregnancy Risk (Low) & -1.05 & .415 \\
\hline & Monetary $(\$ 10)$ & -3.44 & .002 \\
\hline & Monetary $(\$ 100)$ & -0.89 & .453 \\
\hline \multirow[t]{5}{*}{ STD Risk (High) } & STD Risk (Low) & -5.39 & $<.001$ \\
\hline & Pregnancy Risk (High) & -0.50 & .647 \\
\hline & Pregnancy Risk (Low) & -3.85 & $<.001$ \\
\hline & Monetary $(\$ 10)$ & -5.48 & $<.001$ \\
\hline & Monetary $(\$ 100)$ & -3.53 & .001 \\
\hline \multirow[t]{4}{*}{ STD Risk (Low) } & Pregnancy Risk (High) & -5.47 & $<.001$ \\
\hline & Pregnancy Risk (Low) & -2.88 & .030 \\
\hline & Monetary (\$10) & -1.12 & .320 \\
\hline & Monetary $(\$ 100)$ & -1.50 & .139 \\
\hline \multirow[t]{3}{*}{ Pregnancy Risk (High) } & Pregnancy Risk (Low) & -3.55 & .001 \\
\hline & Monetary (\$10) & -5.41 & $<.001$ \\
\hline & Monetary $(\$ 100)$ & -3.22 & .003 \\
\hline \multirow[t]{2}{*}{ Pregnancy Risk (Low) } & Monetary $(\$ 10)$ & -2.97 & .014 \\
\hline & Monetary $(\$ 100)$ & -0.38 & .711 \\
\hline Monetary $(\$ 100)$ & Monetary $(\$ 10)$ & -4.41 & $<.001$ \\
\hline
\end{tabular}




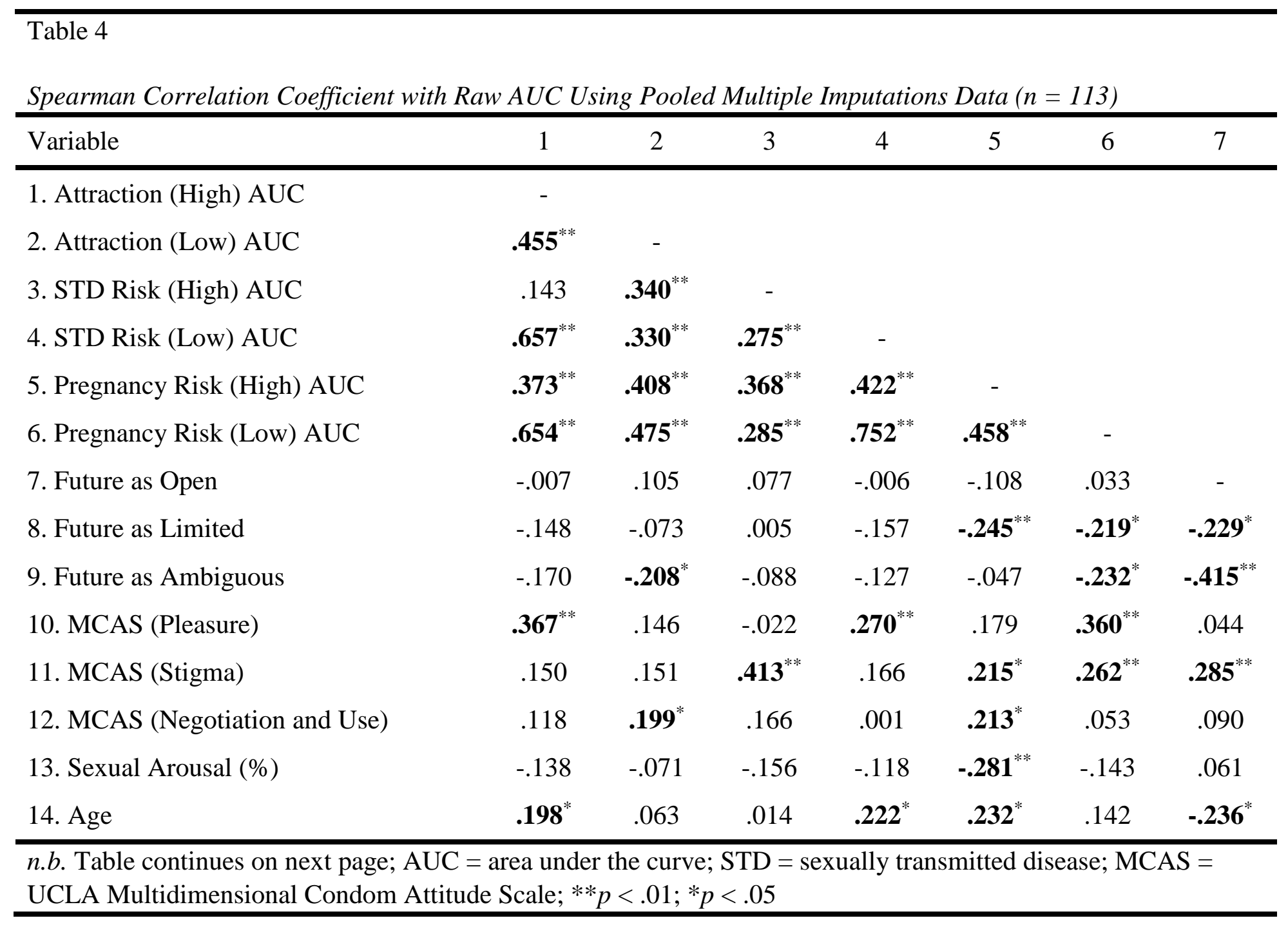




\begin{tabular}{|c|c|c|c|c|c|c|c|}
\hline $\begin{array}{l}\text { Table } 4 \text { (continued) } \\
\text { Spearman Correlation Coefficient }\end{array}$ & Raw $A U$ & $\operatorname{sing} P o$ & Multiple & utations & $a(n=)$ & & \\
\hline Variable & 8 & 9 & 10 & 11 & 12 & 13 & 14 \\
\hline \multicolumn{8}{|l|}{ 1. Attraction (High) AUC } \\
\hline \multicolumn{8}{|l|}{ 2. Attraction (Low) AUC } \\
\hline \multicolumn{8}{|l|}{ 3. STD Risk (High) AUC } \\
\hline \multicolumn{8}{|l|}{ 4. STD Risk (Low) AUC } \\
\hline \multicolumn{8}{|l|}{ 5. Pregnancy Risk (High) AUC } \\
\hline \multicolumn{8}{|l|}{ 6. Pregnancy Risk (Low) AUC } \\
\hline \multicolumn{8}{|l|}{ 7. Future as Open } \\
\hline 8. Future as Limited & - & & & & & & \\
\hline 9. Future as Ambiguous & $.338^{* *}$ & - & & & & & \\
\hline 10. MCAS (Pleasure) & $-.246^{* *}$ & -.116 & - & & & & \\
\hline 11. MCAS (Stigma) & -.109 & -.131 & $.317^{* *}$ & - & & & \\
\hline 12. MCAS (Negotiation and Use) & .0219 & .039 & .133 & $.432^{* *}$ & - & & \\
\hline 13. Sexual Arousal (\%) & .133 & .135 & $-.199^{*}$ & -.144 & .052 & - & \\
\hline 14. Age & -.050 & .014 & .052 & -.037 & -.169 & -.160 & - \\
\hline
\end{tabular}




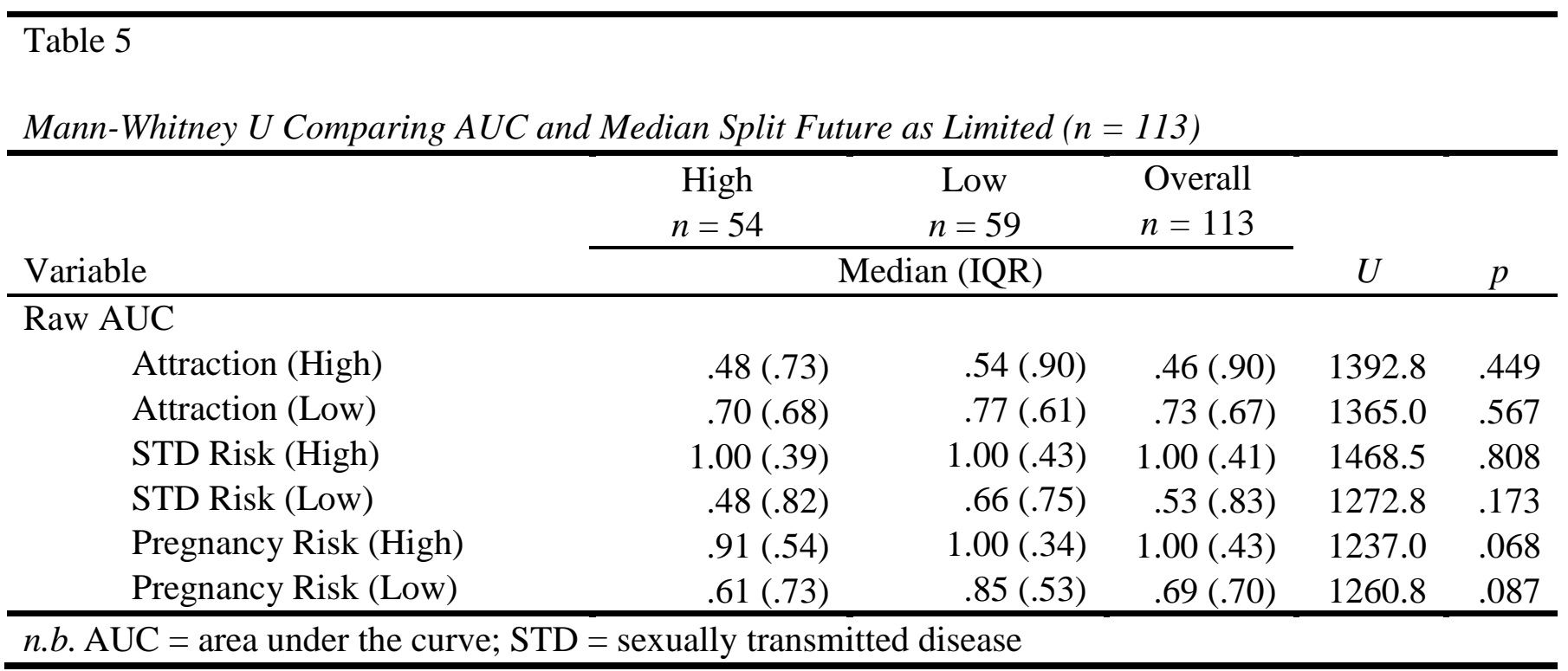




\begin{tabular}{|c|c|c|c|c|c|}
\hline $\begin{array}{l}\text { Table } 6 \\
\text { Mann-Whitney U Comparing A }\end{array}$ & lian Split Fut & as Ambiguous & $n=113)$ & & \\
\hline & $\begin{array}{c}\text { High } \\
n=47\end{array}$ & $\begin{array}{c}\text { Low } \\
n=66\end{array}$ & $\begin{array}{c}\text { Overall } \\
n=113\end{array}$ & & \\
\hline Variable & \multicolumn{3}{|c|}{ Median (IQR) } & $U$ & $p$ \\
\hline \multicolumn{6}{|l|}{ Raw AUC } \\
\hline Attraction (High) & $.47(.72)$ & $.55(.87)$ & $.46(.90)$ & 1276.2 & .228 \\
\hline Attraction (Low) & $.62(.74)$ & $.77(.56)$ & $.73(.67)$ & 1171.0 & .148 \\
\hline STD Risk (High) & $1.00(.39)$ & $1.00(.43)$ & $1.00(.41)$ & 1385.7 & .574 \\
\hline STD Risk (Low) & $.51(.68)$ & $.63(.83)$ & $.53(.83)$ & 1330.8 & .432 \\
\hline Pregnancy Risk (High) & $.94(.41)$ & $1.00(.42)$ & $1.00(.43)$ & 1343.8 & .342 \\
\hline Pregnancy Risk (Low) & $.59(.70)$ & $.86(.53)$ & $.69(.70)$ & 1100.2 & .015 \\
\hline
\end{tabular}




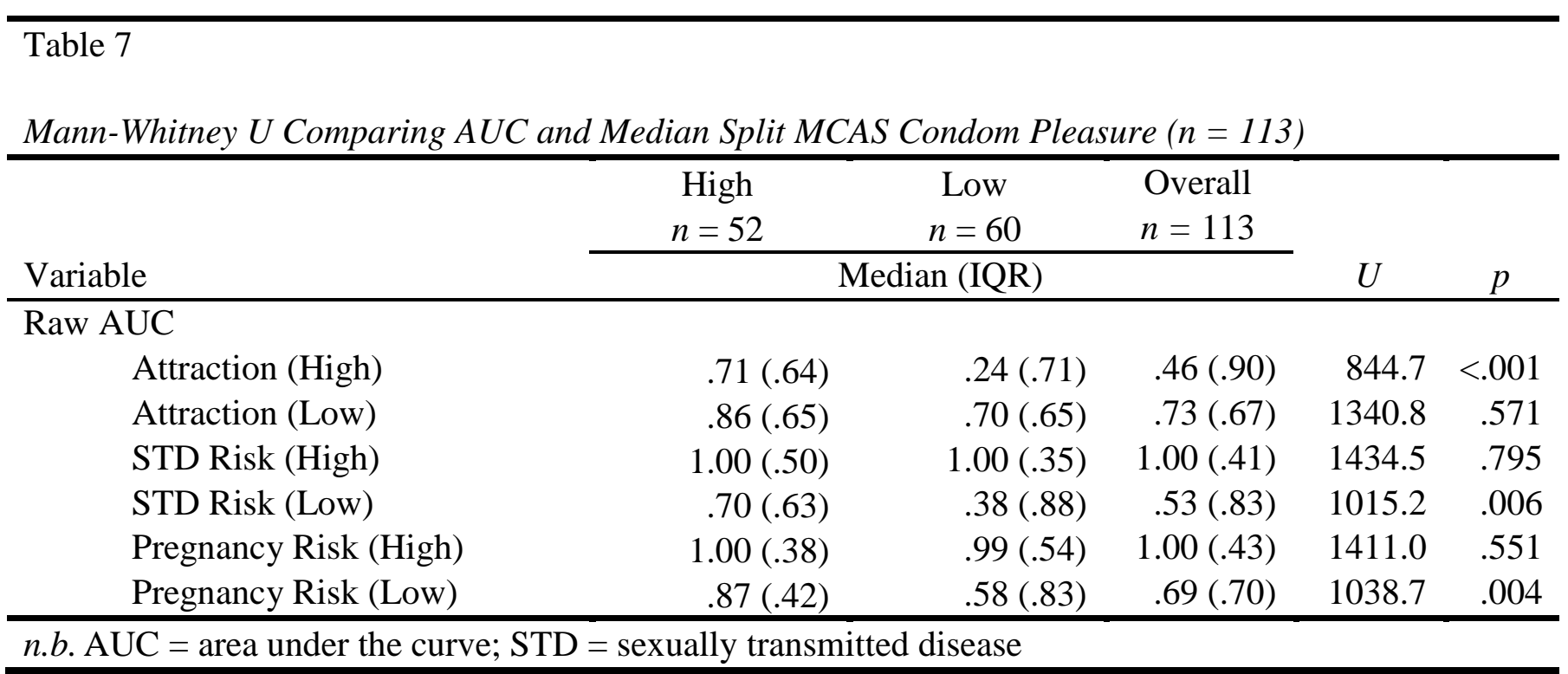




\begin{tabular}{|c|c|c|c|c|c|}
\hline $\begin{array}{l}\text { Table } 8 \\
\text { Mann-Whitney U Comparing A }\end{array}$ & \multicolumn{5}{|c|}{ Table 8} \\
\hline & $\begin{array}{c}\text { High } \\
n=62\end{array}$ & $\begin{array}{c}\text { Low } \\
n=51\end{array}$ & $\begin{array}{l}\text { Overall } \\
n=113\end{array}$ & & \\
\hline Variable & \multicolumn{3}{|c|}{ Median (IQR) } & $U$ & $p$ \\
\hline \multicolumn{6}{|l|}{ Raw AUC } \\
\hline Attraction (High) & $.60(.82)$ & $.36(.78)$ & $.46(.90)$ & 1160.7 & .041 \\
\hline Attraction (Low) & $.79(.54)$ & $.62(.74)$ & $.73(.67)$ & 1148.0 & .068 \\
\hline STD Risk (High) & $1.00(.01)$ & $.80(.60)$ & $1.00(.41)$ & 925.2 & .001 \\
\hline STD Risk (Low) & $.70(.71)$ & $.39(.72)$ & $.53(.83)$ & 1157.3 & .049 \\
\hline Pregnancy Risk (High) & $1.00(.25)$ & $.88(.65)$ & $1.00(.43)$ & 1120.2 & .012 \\
\hline Pregnancy Risk (Low) & $.89(.46)$ & $.53(.74)$ & $.69(.70)$ & 1048.0 & .004 \\
\hline
\end{tabular}




\begin{tabular}{|c|c|c|c|c|c|}
\hline \multicolumn{6}{|l|}{ Table 9} \\
\hline \multicolumn{6}{|c|}{$\begin{array}{l}\text { Mann-Whitney U Comparing AUC and Median Split MCAS Embarrassment About Negotiation and Use } \\
\text { of Condoms }(n=113)\end{array}$} \\
\hline & $\begin{array}{l}\text { High } \\
n=56\end{array}$ & $\begin{array}{c}\text { Low } \\
n=56\end{array}$ & $\begin{array}{l}\text { Overall } \\
n=113\end{array}$ & \multirow[b]{2}{*}{$U$} & \multirow[b]{2}{*}{$p$} \\
\hline Variable & \multicolumn{3}{|c|}{ Median (IQR) } & & \\
\hline \multicolumn{6}{|l|}{ Raw AUC } \\
\hline Attraction (High) & $.59(.85)$ & $.43(.77)$ & $.46(.90)$ & 1345.0 & .378 \\
\hline Attraction (Low) & $.75(.54)$ & $.70(.72)$ & $.73(.67)$ & 1241.8 & .226 \\
\hline STD Risk (High) & $1.00(.39)$ & $1.00(.49)$ & $1.00(.41)$ & 1395.0 & .566 \\
\hline STD Risk (Low) & $.51(.74)$ & $.63(.77)$ & $.53(.83)$ & 1302.7 & .302 \\
\hline Pregnancy Risk (High) & $1.00(.28)$ & $.89(.55)$ & $1.00(.43)$ & 1231.2 & .078 \\
\hline Pregnancy Risk (Low) & $.76(.72)$ & $.70(.62)$ & $.69(.70)$ & 1435.8 & .584 \\
\hline
\end{tabular}




\begin{tabular}{|c|c|c|c|c|c|}
\hline \multicolumn{6}{|l|}{ Table 10} \\
\hline \multirow[b]{2}{*}{ Variable } & $\begin{array}{c}\text { High } \\
n=56\end{array}$ & $\begin{array}{c}\text { Low } \\
n=57\end{array}$ & $\begin{array}{c}\text { Overall } \\
n=113\end{array}$ & \multirow[b]{2}{*}{$U$} & \multirow[b]{2}{*}{$p$} \\
\hline & \multicolumn{3}{|c|}{ Median (IQR) } & & \\
\hline \multicolumn{6}{|l|}{ Raw AUC } \\
\hline Attraction (High) & $.48(.75)$ & $.59(.87)$ & $.46(.90)$ & 1315.2 & .223 \\
\hline Attraction (Low) & $.67(.70)$ & $.78(.59)$ & $.73(.67)$ & 1344.7 & .491 \\
\hline STD Risk (High) & $1.00(.51)$ & $1.00(.30)$ & $1.00(.41)$ & 1383.2 & .435 \\
\hline STD Risk (Low) & $.53(.79)$ & $.61(.75)$ & $.53(.83)$ & 1406.5 & .574 \\
\hline Pregnancy Risk (High) & $.88(.58)$ & $1.00(.28)$ & $1.00(.43)$ & 1178.8 & .029 \\
\hline Pregnancy Risk (Low) & $.69(.64)$ & $.85(.67)$ & $.69(.70)$ & 1424.7 & .448 \\
\hline
\end{tabular}




\begin{tabular}{|c|c|c|c|c|c|}
\hline \multicolumn{6}{|l|}{ Table 11} \\
\hline \multicolumn{6}{|c|}{ Mann-Whitney U Comparing AUC and Gender $(n=113)$} \\
\hline & $\begin{array}{c}\text { Women } \\
n=83\end{array}$ & $\begin{array}{c}\text { Men } \\
n=30\end{array}$ & $\begin{array}{c}\text { Overall } \\
n=113\end{array}$ & \multirow[b]{2}{*}{$U$} & \multirow[b]{2}{*}{$p$} \\
\hline Variable & \multicolumn{3}{|c|}{ Median (IQR) } & & \\
\hline \multicolumn{6}{|l|}{ Raw AUC } \\
\hline Attraction (High) & $.54(.90)$ & $.34(.90)$ & $.49(.90)$ & 1024.0 & .307 \\
\hline Attraction (Low) & $.78(.62)$ & $.58(.69)$ & $.73(.67)$ & 927.0 & .220 \\
\hline STD Risk (High) & $1.00(.40)$ & $1.00(.50)$ & $1.00(.41)$ & 1084.3 & .500 \\
\hline STD Risk (Low) & $.58(.80)$ & $.34(.88)$ & $.56(.83)$ & 960.2 & .176 \\
\hline Pregnancy Risk (High) & $1.00(.37)$ & $.89(.87)$ & $1.00(.43)$ & 951.2 & .096 \\
\hline Pregnancy Risk (Low) & $.82(.61)$ & $.57(.87)$ & $.72(.70)$ & 940.2 & .085 \\
\hline
\end{tabular}




\begin{tabular}{|c|c|c|c|c|c|}
\hline \multicolumn{6}{|l|}{ Table 12} \\
\hline \multicolumn{6}{|c|}{ Mann-Whitney U Comparing AUC and Reproductive-aged Participants $(n=74)$} \\
\hline & $\begin{array}{c}\text { Women } \\
n=44\end{array}$ & $\begin{array}{c}\text { Men } \\
n=30\end{array}$ & $\begin{array}{c}\text { Overall } \\
n=74\end{array}$ & & \\
\hline Variable & \multicolumn{3}{|c|}{ Median (IQR) } & $U$ & $p$ \\
\hline \multicolumn{6}{|l|}{ Raw AUC } \\
\hline Attraction (High) & $.44(.68)$ & $.34(.90)$ & $.41(.77)$ & 601.3 & .814 \\
\hline Attraction (Low) & $.71(.66)$ & $.58(.69)$ & $.68(.71)$ & 506.2 & .349 \\
\hline STD Risk (High) & $1.00(.53)$ & $1.00(.50)$ & $1.00(.49)$ & 605.5 & .822 \\
\hline STD Risk (Low) & $.37(.83)$ & $.34(.88)$ & $.36(.89)$ & 573.0 & .604 \\
\hline Pregnancy Risk (High) & $.97(.46)$ & $.89(.87)$ & $.93(.52)$ & 531.8 & .243 \\
\hline Pregnancy Risk (Low) & $.63(.70)$ & $.57(.87)$ & $.62(.77)$ & 570.8 & .457 \\
\hline
\end{tabular}




\begin{tabular}{|c|c|c|c|c|c|}
\hline $\begin{array}{l}\text { Table } 13 \\
\text { Mann-Whitney U Comparing A }\end{array}$ & elationship Stat & $(n=113)$ & & & \\
\hline & $\begin{array}{c}\text { In a } \\
\text { relationship } \\
n=31\end{array}$ & $\begin{array}{l}\text { Not in a } \\
\text { relationship } \\
n=82\end{array}$ & $\begin{array}{l}\text { Overall } \\
n=113\end{array}$ & \multirow[b]{2}{*}{$U$} & \multirow[b]{2}{*}{$p$} \\
\hline Variable & \multicolumn{3}{|c|}{ Median (IQR) } & & \\
\hline \multicolumn{6}{|l|}{ Raw AUC } \\
\hline Attraction (High) & $.53(.91)$ & $.49(.88)$ & $.49(.90)$ & 1177.2 & .777 \\
\hline Attraction (Low) & $.67(.73)$ & $.75(.64)$ & $.73(.67)$ & 1125.2 & .808 \\
\hline STD Risk (High) & $1.00(.39)$ & $1.00(.42)$ & $1.00(.41)$ & 1185.8 & .884 \\
\hline STD Risk (Low) & $.39(.79)$ & $.59(.82)$ & $.56(.83)$ & 1054.5 & .318 \\
\hline Pregnancy Risk (High) & $1.00(.52)$ & $.98(.43)$ & $1.00(.43)$ & 1205.3 & .868 \\
\hline Pregnancy Risk (Low) & $.73(.59)$ & $.72(.77)$ & $.72(.70)$ & 1103.3 & .380 \\
\hline
\end{tabular}

n.b. AUC = area under the curve; STD = sexually transmitted disease; participants who responded

"married" and "separated" were combined to create a variable representing participants that are "in a relationship"; participants who responded, "never married", "divorced", "living with a partner", and "widowed" were combined to create a variable representing participants that are "not in a relationship" 
Figure 1. Sexual Discounting Task data orderliness.

\begin{tabular}{|c|}
\hline $\begin{array}{c}\text { Total Consented Participants } \\
\mathrm{N}=128\end{array}$ \\
\hline $\begin{array}{c}\text { Incomplete Data } \\
n=6\end{array}$ \\
\hline Randomization Error (Missing Pregnancy Unlikely) \\
$n=9$
\end{tabular}

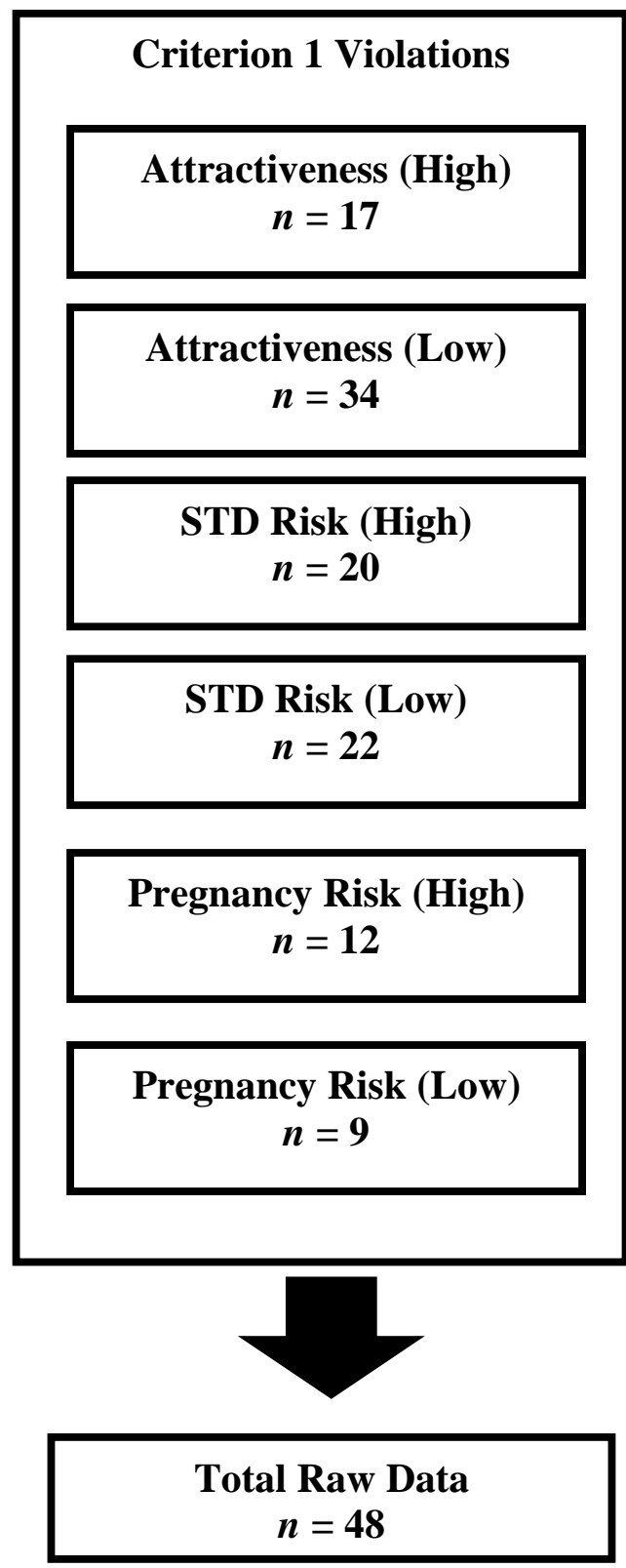


Figure 2. Monetary Delay-Discounting Task data orderliness.

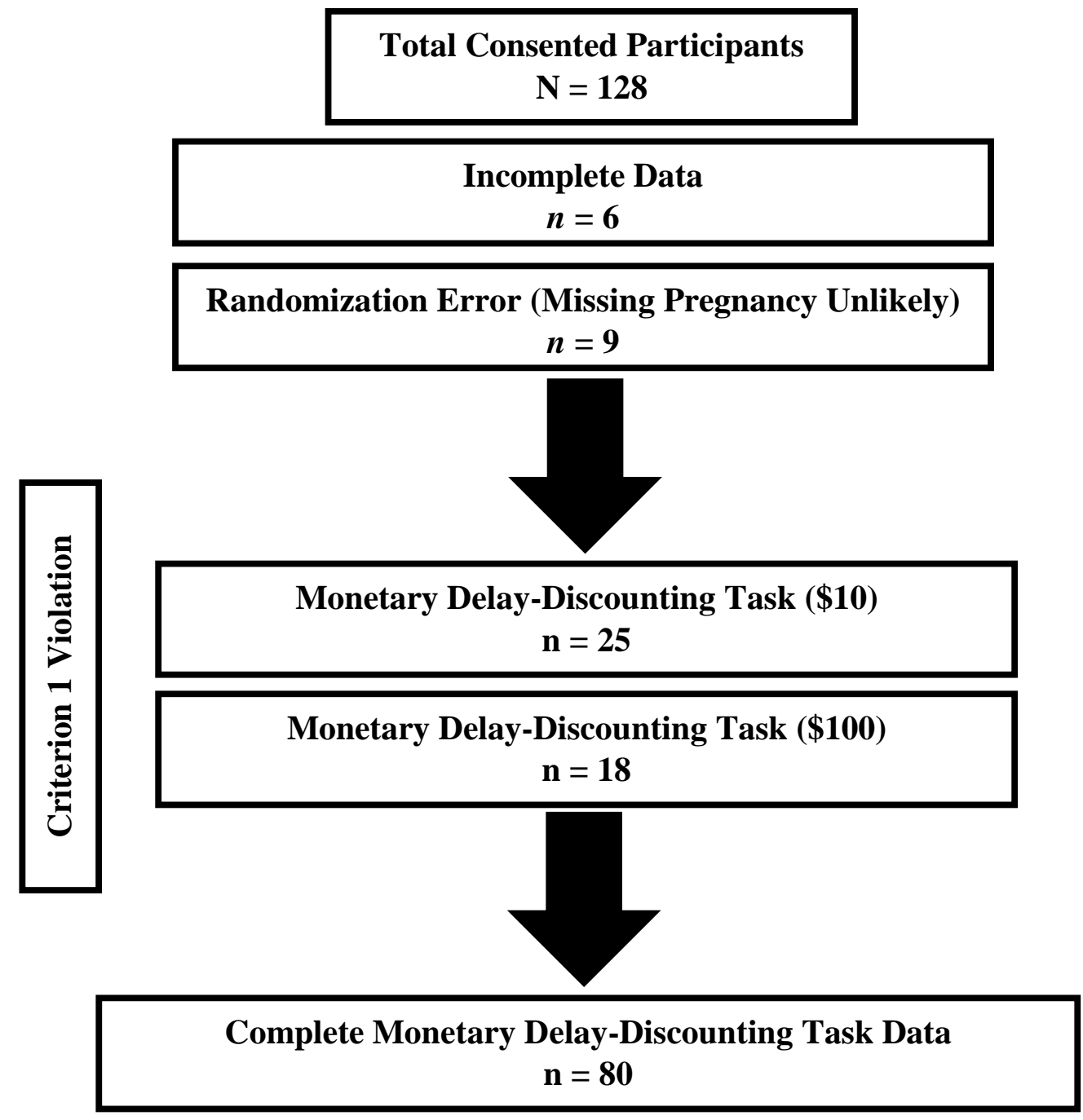


Figure 3. Illustrating the computation of AUC using sample data.

\section{Computing Area Under the Curve}

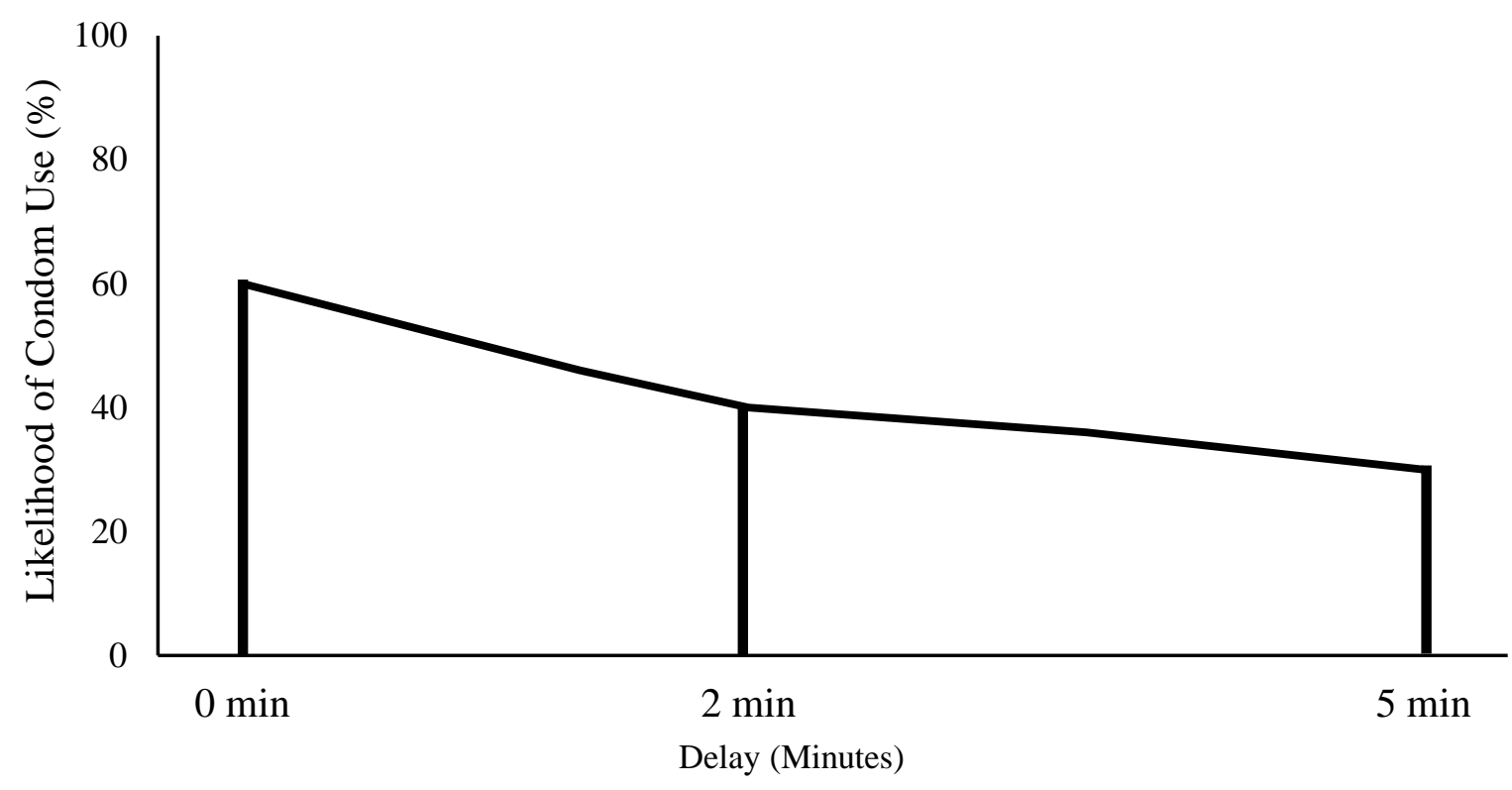


Figure 4. Sample participant data with Criterion 1 violations.

Characteristic Non-systematic Data (Criterion 1)

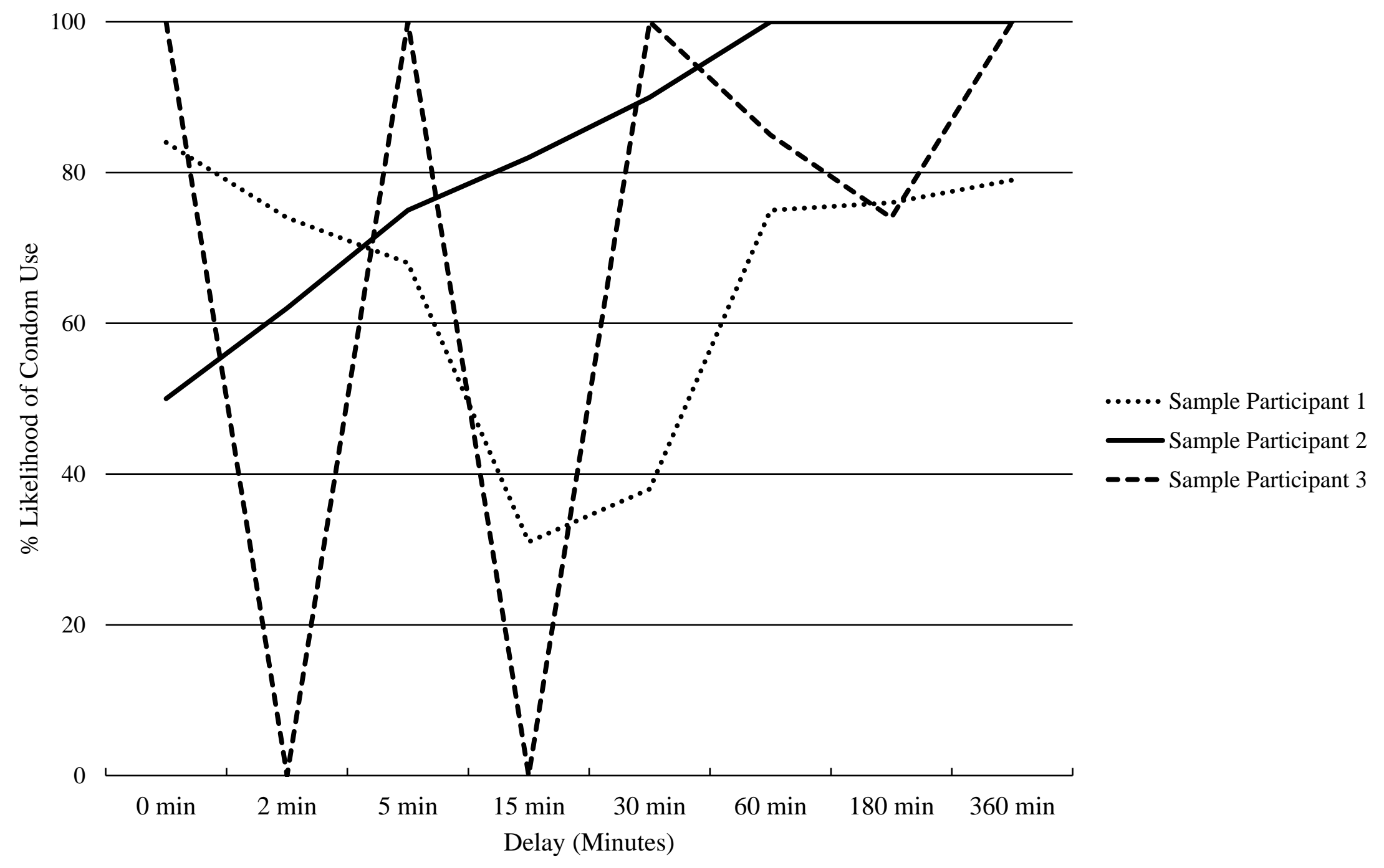


Figure 5. Monetary Delay Discounting Task Means

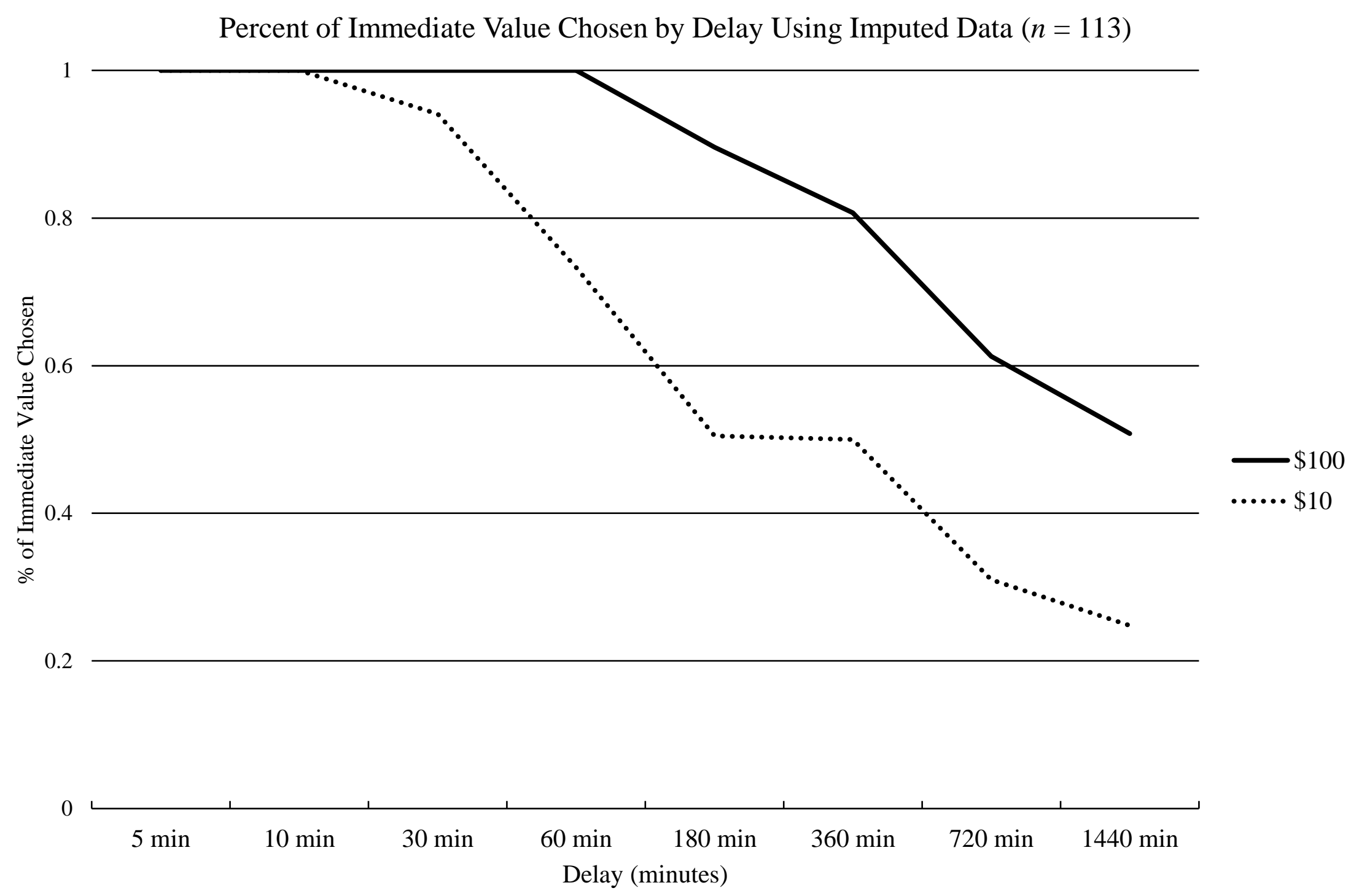


Figure 6. Sexual Discounting Task Means

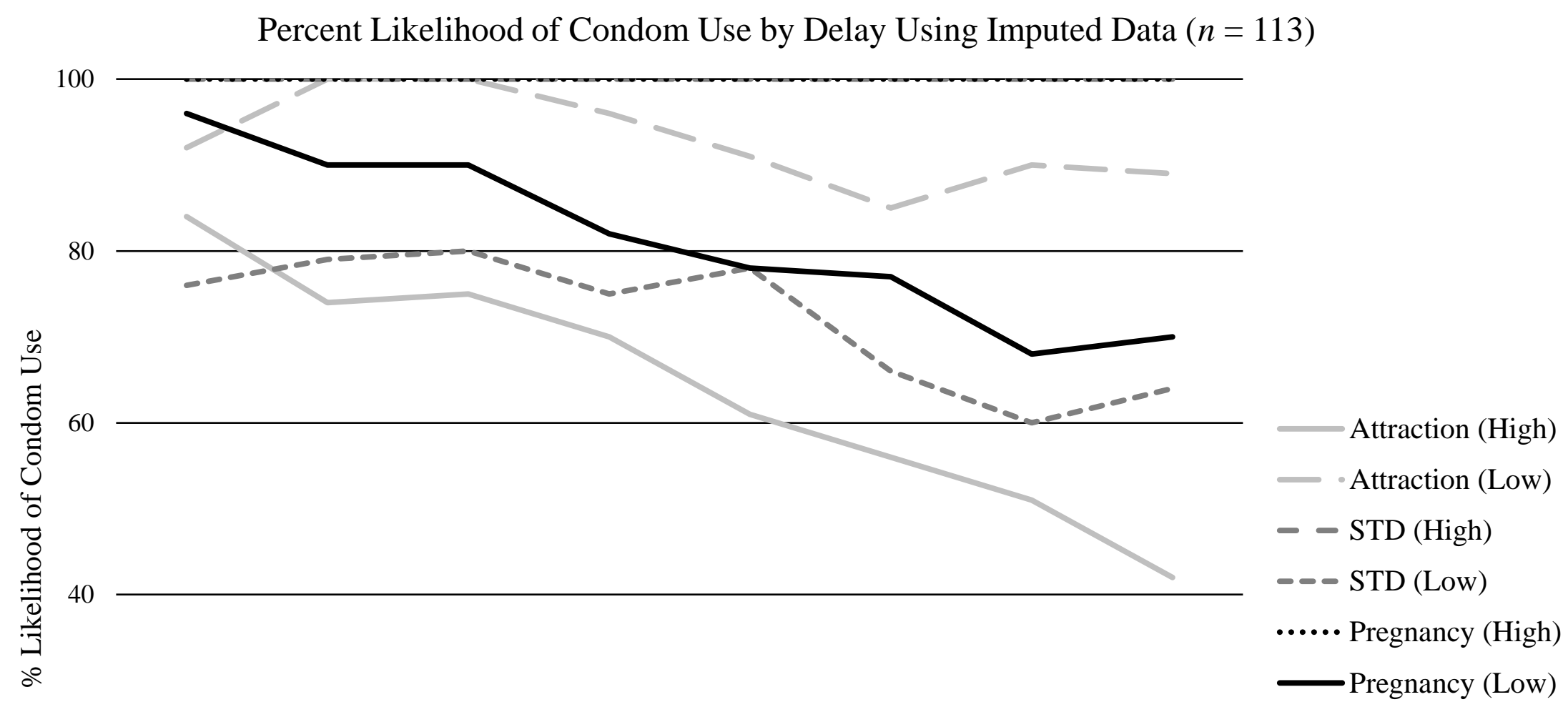

20

\begin{tabular}{|c|c|c|c|c|c|c|c|}
\hline $0 \mathrm{~min}$ & $2 \min$ & $5 \min$ & $\begin{array}{r}15 \text { min } \\
\text { Delay }\end{array}$ & $\begin{array}{l}30 \mathrm{~min} \\
\text { inutes) }\end{array}$ & $60 \mathrm{~min}$ & $180 \mathrm{~min}$ & $360 \mathrm{~min}$ \\
\hline
\end{tabular}




\section{Appendix A}

\section{WestVirginiaUniversitys Human Research Protocol OFFICE OF RESEARCH IIITEGRIT S COMPLIANCE
(With HIPAA)}

\section{Only Minimal Risk Consent Information and HIPAA Form}

$\begin{array}{ll}\begin{array}{l}\text { Principal Investigator } \\ \text { Department }\end{array} & \begin{array}{l}\text { Laura Lander, MSW, LICSW } \\ \text { Behavioral Medicine and Psychiatry } \\ \text { Protocol Number }\end{array} \\ \text { Study Title } & \begin{array}{l}\text { Comprehensive Opioid Addiction Treatment Reproductive Health } \\ \text { Study }\end{array} \\ \text { Co-Investigator(s) } & \begin{array}{l}\text { Julie Hicks Patrick, Ph.D.; Jonathan JK Stoltman, M.A.; } \\ \text { Valerie K. Blake, M.S.; Sara C. Warfield, M.P.H.; Boglarka Vizy, M.A.; } \\ \text { Michaela S. Reardon, M.A.; Tanisha M. Hendrix }\end{array} \\ \text { Sponsor (if any) } & \text { WVUCTSI U54GM104942 }\end{array}$

\section{Contact Persons}

In the event you experience any side effects or injury related to this research, you should contact Laura Lander at (304) 293-3965. (After hours contact: Laura Lander at (304)598-4214). If you have any questions, concerns, or complaints about this research, you can contact Laura Lander at (304) 293-3965.

For information regarding your rights as a research subject, to discuss problems, concerns, or suggestions related to the research, to obtain information or offer input about the research, contact the Office of Research Compliance at (304) 293-7073.

In addition, if you would like to discuss problems, concerns, have suggestions related to research, or would like to offer input about the research, contact the Office of Research Integrity and Compliance at 304-293-7073.

Introduction

You, _L have been asked to participate in this research study, which has been explained to you by ___. This study is being conducted by Laura Lander in the Department of Behavioral Medicine and Psychiatry, as well as Julie Hicks Patrick and Jonathan Stoltman in the Department of Psychology at West Virginia University. Valerie Blake, Boglarka Vizy, Michaela Reardon, and Tanisha Hendrix in the Department of Psychology and Sara Warfield in the School of Public Health at West Virginia University will also be assisting with the study. Funding was provided by the West Virginia Clinical Translational Science Institute and the West Virginia University Department of Psychology.

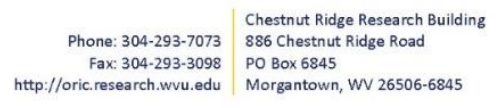

Approved:28-Aug-2017Expires:16-May-2018Number:1705577322 


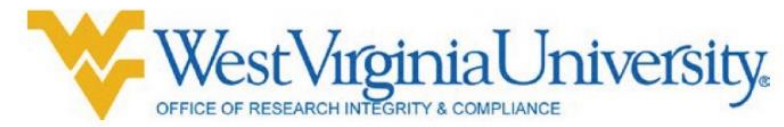

OFFICE OF RESEARCH INTEGRITY \& COMPLIANCE
Human Research Protocol

Only Minimal Risk Consent Form

(With HIPAA)

\section{Purpose(s) of the Study}

The purpose of this study is to learn more about reproductive health behaviors in individuals enrolled in medication assisted treatment for opioid use disorders. We hope you can share with us: what birth control you use or would like to use, how hard it is to see your doctor, how you feel about using birth control in general, and how well you understand what your doctors tell you. With this information we want to better understand how to help you and how to guide other clinics like this toward improving patient reproductive health needs. This study will be conducted at Chestnut Ridge Center in Morgantown WW. We expect to enroll approximately 150 females and 100 male participants.

\section{Description of Procedures}

This study involves surveying reproductive health behaviors of people in opioid maintenance treatment. You are being asked to participate in this study to see if there are any differences in health literacy, access to birth control, birth control use patterns, and menstrual health among women or erectile function in men in opioid maintenance treatment. The aim of this study is to better understand the high rate of unplanned pregnancies observed in other opioid maintenance clinic settings. You will be asked to complete a survey that will be administered using tablet computers, at three different time points. Each survey will take approximately 15 minutes to complete.

The initial survey will ask about health literacy, birth control use type, birth control access, reproductive health, attitudes towards birth control use, time perspective, and personality. A second survey at a later date will ask questions about STI risk evaluation and STI testing. The final survey will follow-up on questions in the first survey in addition to questions on general health and substance use initiation. At the end of the final survey, participants will be provided resource pamphlets for the Rape and Domestic Violence Information Center. Additionally, those requesting further consultation regarding this will be put in contact with their group leader or study Co-Investigator Laura Lander, depending on participant preference.

You do not have to answer all the questions. You will have the opportunity to see the survey before signing this consent form.

For those interested in taking survey 2 or 3 , an additional question has been added to the survey to determine your willingness to be re-contact for those portions of the study. Re-contact will take place before your COAT clinic meeting and only for those interested in continued participation. We will use your anonymous participant ID number from survey 1 to securely connect data across the three surveys.

At the end of each survey wave, you will be given the opportunity to schedule an appointment at WVU hospitals or in your local community to have a consultation regarding contraception and your reproductive health. If you are interested in an appointment, study staff will help you schedule one. To do so they will need your full name and date of birth. This data will not be recorded as part of the study data and will only be used for purpose of helping you make an appointment.

We will check to see if you attend this appointment if one was made at WV. If you do not attend there will be no penalty for you. Attendance at this appointment is voluntary.

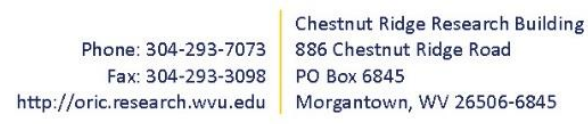

Phone: $304-293-7073$ http://oric. research.wvu.ed

\author{
Morgantown, WV 26506-6845
}

$P a g$ e 12

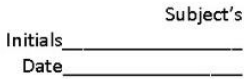




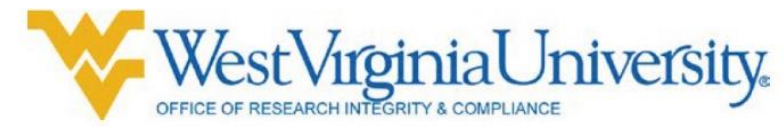

Human Research Protocol
Only Minimal Risk Consent Form
(With HIPAA)

\section{Discomforts}

There are no risks as a control participant in this study. Participants sometimes experience mild frustration associated with answering the questions on the surveys. If during answering any study questions a patient should become upset or there is an identified need for therapeutic intervention, the patient will be asked for permission to contact the patient's individual or group therapist for follow up with the patient and therapeutic services will be provided. If the patient would like a consultation with a different provider than one they already see, co-investigator Laura Lander will be contacted and will facilitate the request. Additionally, all participants will be provided resource pamphlets from the Rape and Domestic Violence Information Center upon completing wave 3 of the study.

\section{Alternatives}

You do not have to participate in this study. There are no alternatives to participation.

\section{Benefits}

The knowledge gained from this study may eventually benefit others. You will have the opportunity to access contraceptive resources as part of participation in the study. If you choose not to participate in the study, you can also access those resources.

\section{Financial Considerations}

There are no fees for participating in this study. The expense of participating in the COAT program will continue to be billed to your insurance company. The cost of attending and appointment for a consultation regarding contraception and your reproductive health will also be billed to your insurance. You will be responsible for any co-pay associated with your appointment if your insurance requires that.

You will be paid $\$ 10$ in the form of a gift card when you complete the first survey. The second survey and final survey will be paid $\$ 15$ and $25 \$$ respectively. If you participate in all 3 study waves, your total payment for completing the study is $50 \$$.

\section{Confidentiality}

Any information about you that is obtained as a result of your participation in this research will be kept as confidential as legally possible. Your research records and test results, just like hospital records, may be subpoenaed by court order or may be inspected by the study sponsor or federal regulatory authorities without your additional consent.

In any publications that result from this research, neither your name nor any information from which you might be identified will be published without your consent.

\section{HIPAA}

We know that information about you and your health is private. We are dedicated to protecting the privacy of that information. Because of this promise, we must get your written authorization (permission) before we may use or disclose your protected health information or share it with others for research purposes.

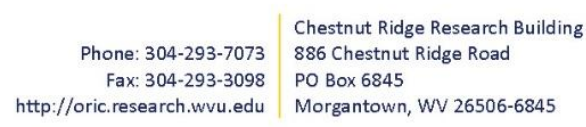

$$
\text { Page } 13
$$

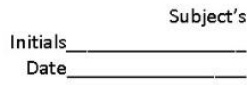




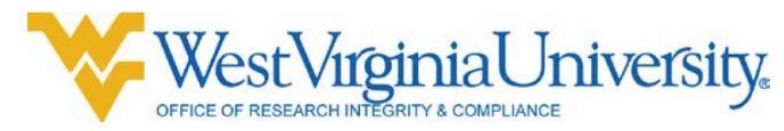

Human Research Protocol
Only Minimal Risk Consent Form
(With HIPAA)

You can decide to sign or not to sign this authorization section. However, if you choose not to sign this authorization, you will not be able to take part in the research study. Whatever choice you make about this research study will not have an effect on your access to medical care.

\section{Persons/Organizations Providing the Information}

Patient/West Virginia University Hospitals

Persons/Organizations Receiving the Information

- The research site(s) carrying out this study. This includes UHA or UHA Affiliated, WWU, WVU Hospitals. It also includes each site's research staff and medical staff

- Health care providers who provide services to you as part of this research study.

- The members and staff of any Institutional Review Board (IRB) that oversees this research study.

- West Virginia University Office of Research Compliance and Office of Sponsored Programs.

The Following Information Will Be Used

Only information that you provide to us on the study survey will be used in the study. We will also follow up and see if you were able to attend your appointment if you made one at Ruby Hospital OBGYN for consultation regarding contraception and your reproductive health. Information from your Chestnut Ridge medical chart including: start date at the clinic, days clean or days since last used substances at date of participation, opioid use disorder diagnosis, mental health diagnoses history, and referral source.

\section{The Information is Being Disclosed for the Following Reasons}

- Review of your data for quality assurance purposes

- $\quad$ Publication of study results (without identifying you)

- Other research purposes such as reviewing the safety or effectiveness of the study therapies; evaluating therapies for patients; developing a better understanding of disease; improving the design of future clinical trials

\section{You May Cancel this Authorization at Any Time by Writing to the Principal Investigator}

Laura R. Lander, MSW, LICSW

Assistant Professor

Chestnut Ridge Center

930 Chestnut Ridge Road

Morgantown WW, 26505

West Virginia University

304-293-3965

llander@hsc.wvu.edu

If you cancel this authorization, any information that was collected already for this study cannot be withdrawn. Once information is disclosed, according to this authorization, the recipient may redisclose it and then the information may no longer be protected by federal regulations.

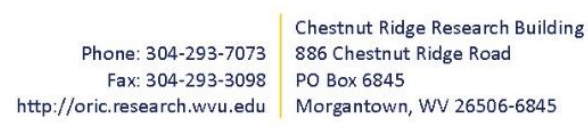

$P a g$ e 14

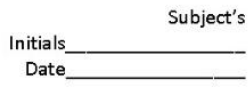




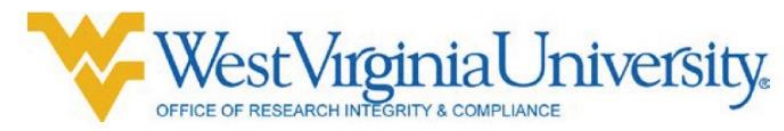

Human Research Protocol

Only Minimal Risk Consent Form

(With HIPAA)

You have a right to see and make copies of your medical records. You will not be able to see or copy your records related to the study until the sponsor has completed all work related to the study. At that time you may ask to see the study doctor's files related to your participation in the study and have the study doctor correct any information about you that is wrong.

This authorization will expire at the end of the study unless you cancel it before that time.

\section{Voluntary Participation}

Participation in this study is voluntary. You are free to withdraw your consent to participate in this study at any time.

Refusal to participate or withdrawal will not affect your treatment and will involve no penalty to you. Refusal to participate or withdrawal will not affect your future care.

In the event new information becomes available that may affect your willingness to participate in this study, this information will be given to you so that you can make an informed decision about whether or not to continue your participation.

You have been given the opportunity to ask questions about the research, and you have received answers concerning areas you did not understand.

Upon signing this form, you will receive a copy.

I willingly consent to participate in this research.

\section{Signatures}

Signature of Subject

\begin{tabular}{lll}
\hline Printed Name & Date & Time
\end{tabular}

The participant has had the opportunity to have questions addressed. The participant willingly agrees to be in the study.

Signature of Investigator or Co-Investigator

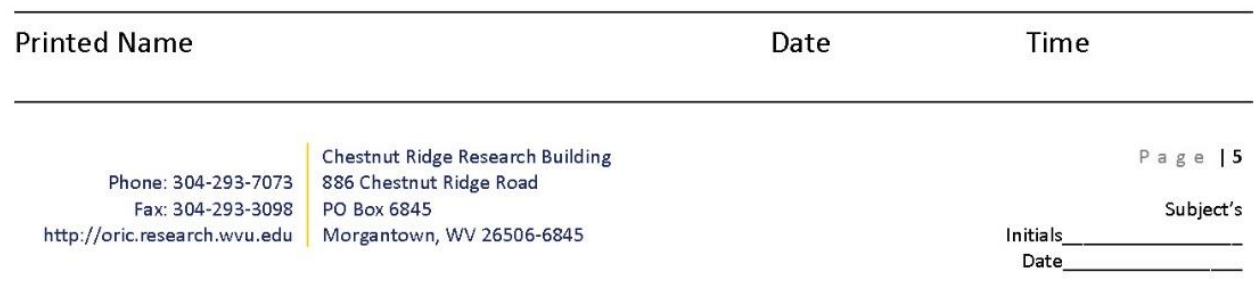


Confidential

Page 2 of 42

Thank you for agreeing to take part in this survey on reproductive health at the COAT clinic. Please answer as
truthfully and accurately as possible. All responses will be kept confidential.
Date subject signed consent
(MM-DD-YYYY)
I consent to the following study
Yes
No




\section{Confidential}

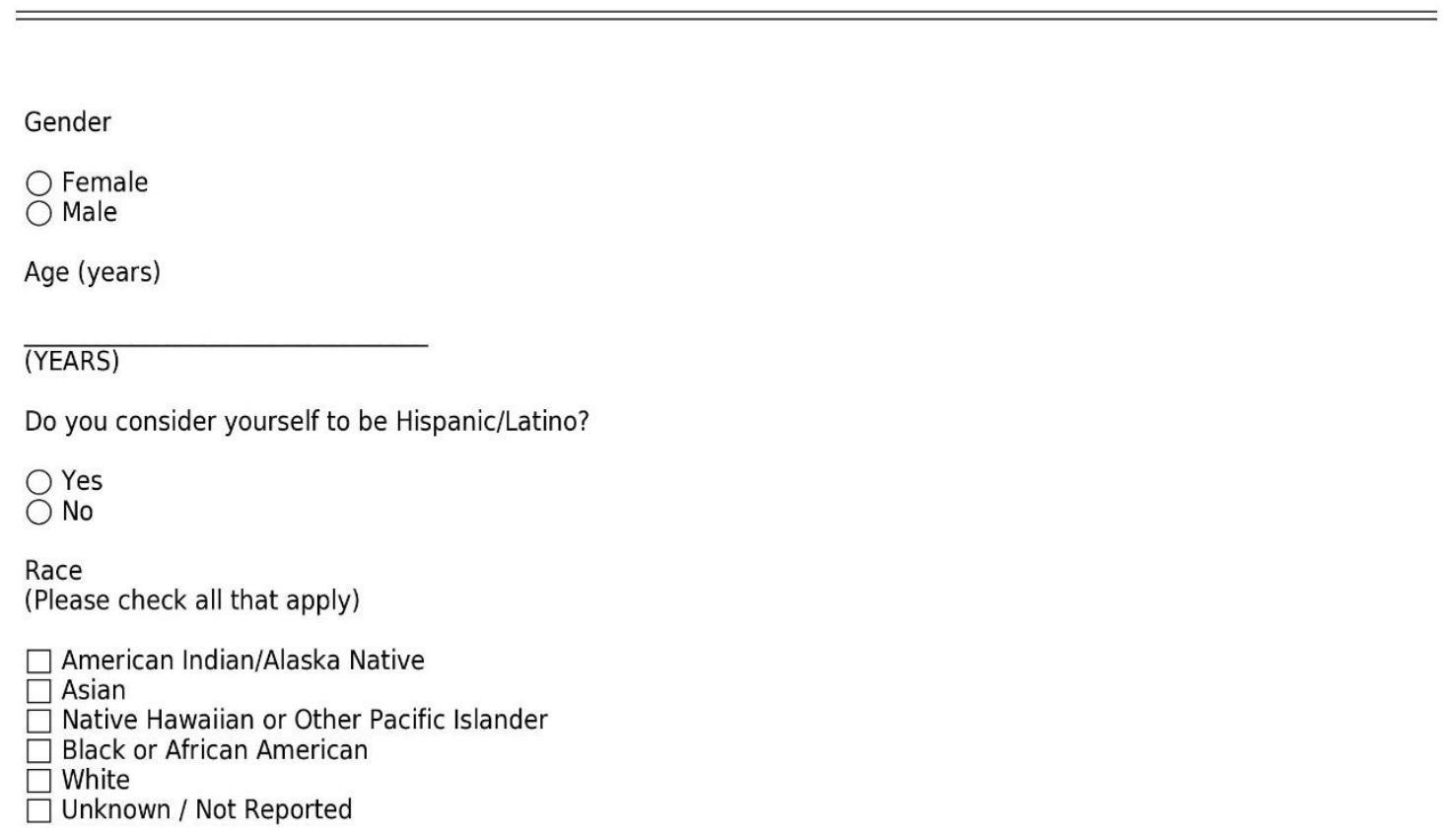


Confidential

Page 4 of 42

$=$

How tall are you?

Feet

Inches

How much do you weigh (in kilograms)?

(KILOGRAMS)

Years of education?

(For example - completed high school or GED $=12$ years) 


\section{Confidential}

Time Perspective (pt. 1)

Please answer the following questions about how you feel about the future and your general personality traits

I look forward to the future with hope and enthusiasm

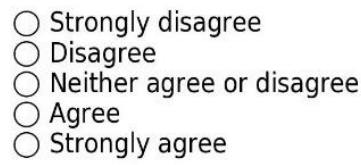

When I think about the future, I expect good things to happen

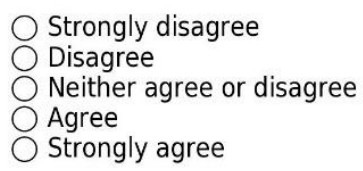

I have little hope for the future

Strongly disagree

Disagree

Neither agree or disagree

Agree

Strongly agree

Each new day and season presents me with interesting opportunities

$\bigcirc$ Strongly disagree

Disagree

Neither agree or disagree

Agree

$\bigcirc$ Strongly agree

I have the sense that time is running out

Strongly disagree

Disagree

Neither agree or disagree

Agree

$\bigcirc$ Strongly agree

I am beginning to experience that time is limited

Strongly disagree

Disagree

Neither agree or disagree

Agree

Strongly agree 


\section{Confidential}

Time Perspective (pt. 2)

Please answer the following questions about how you feel about the future and your general personality traits

Increasingly I feel like time is against me

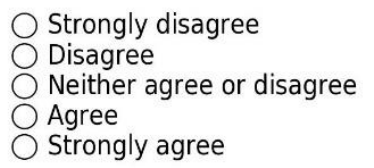

I know that I do not have all the time in the world

Strongly disagree

$\bigcirc$ Disagree

Neither agree or disagree

Agree

$\bigcirc$ Strongly agree

My future is uncertain

Strongly disagree

Disagree

Neither agree or disagree

Agree

Strongly agree

You cannot really plan for the future because things change so much

$\bigcirc$ Strongly disagree

Disagree

Neither agree or disagree

Agree

$\bigcirc$ Strongly agree

My future seems very vague and uncertain to me

Strongly disagree

$\bigcirc$ Disagree

Neither agree or disagree

Agree

$\bigcirc$ Strongly agree

I do not focus on the future because it is so uncertain to me anyway

Strongly disagree

Disagree

Neither agree or disagree

Agree

Strongly agree 


\section{Confidential}

Page 80 of 80

\section{Section Break}

We would like to contact you in the future to see if you would be interested in participating in another part of THIS sexual health research study.

Please indicated if you would be willing to be contacted about future parts of this study:

Yes, I agree to be contacted

$\bigcirc$ No, I don't want to be contacted further

Thank you for participating in this survey!

Is there anything else you think we should consider regarding women's health at the COAT clinic? 


\section{Confidential}

\section{Condom Pleasure}

The use of condoms can make sex more stimulating

Strongly disagree

$\bigcirc$ Disagree

$\bigcirc$ Somewhat disagree

Neither agree or disagree

$\bigcirc$ Somewhat agree

Agree

Strongly agree

Condoms ruin the sex act

Strongly disagree

Disagree

$\bigcirc$ Somewhat disagree

Neither agree or disagree

$\bigcirc$ Somewhat agree

Agree

$\bigcirc$ Strongly agree

Condoms are uncomfortable for both partners

Strongly disagree

$\bigcirc$ Disagree

$\bigcirc$ Somewhat disagree

Neither agree or disagree

$\bigcirc$ Somewhat agree

Agree

$\bigcirc$ Strongly agree

Condoms are a lot of fun

Strongly disagree

$\bigcirc$ Disagree

$\bigcirc$ Somewhat disagree

Neither agree or disagree

Somewhat agree

Agree

Strongly agree

Use of a condom is an interruption of foreplay

Strongly disagree

Disagree

$\bigcirc$ Somewhat disagree

Neither agree or disagree

$\bigcirc$ Somewhat agree

Agree

Strongly agree 


\section{Confidential}

\section{Identity Stigma}

Men who suggest using a condom are really boring

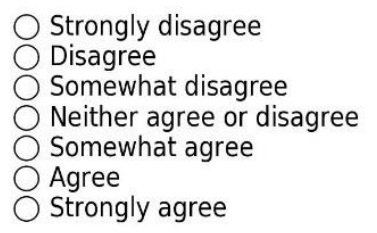

If a couple is about to have sex and the man suggests using a condom, it is less likely that they will have sex

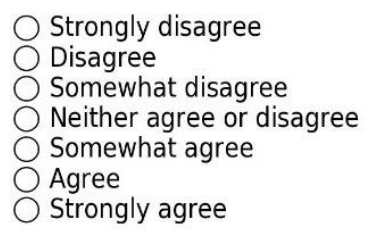

Women think men who use condoms are jerks

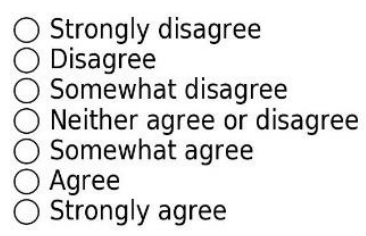

A woman who suggests using a condom does not trust her partner

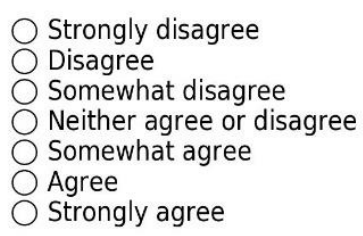

People who suggest condom use are a little bit geeky

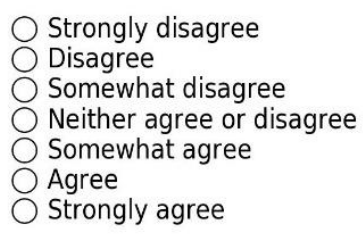




\section{Confidential}

\section{Negotiation and Use}

When I suggest using a condom I am almost always embarrassed

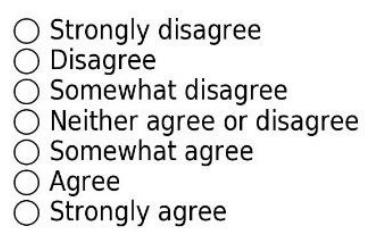

It is really hard to bring up the issue of using condoms to my partner

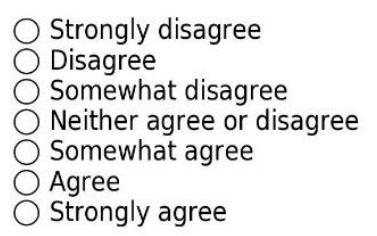

It is easy to suggest to my partner that we use a condom

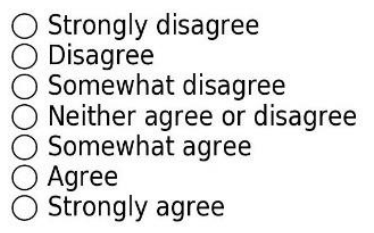

I'm comfortable talking about condoms with my partner

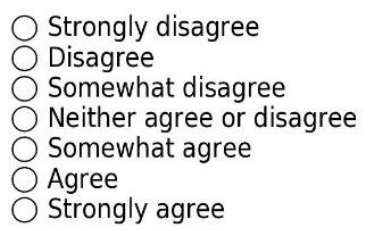

I never know what to say when my partner and I need to talk about condoms or other protection

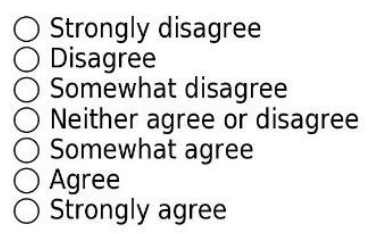




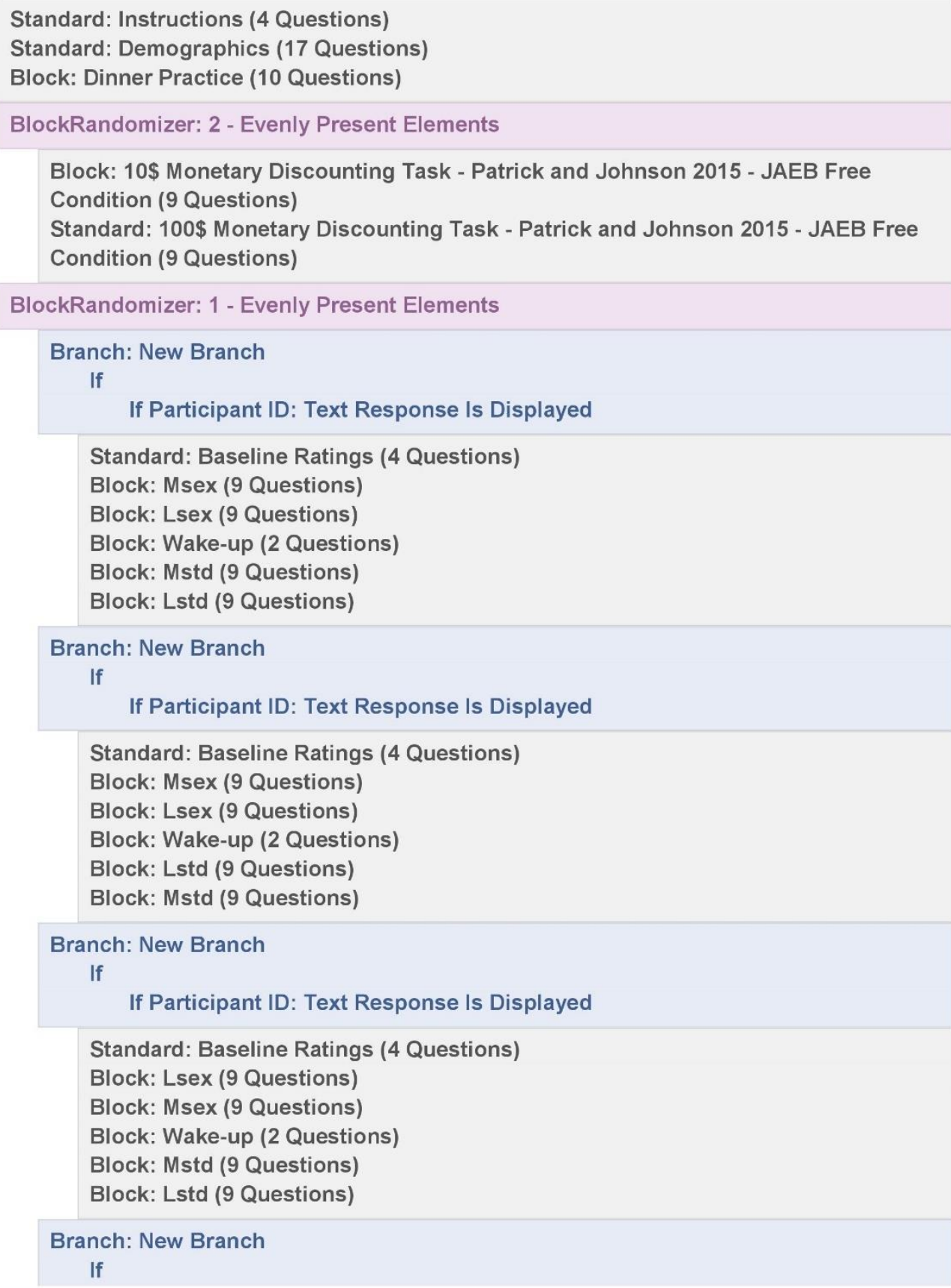




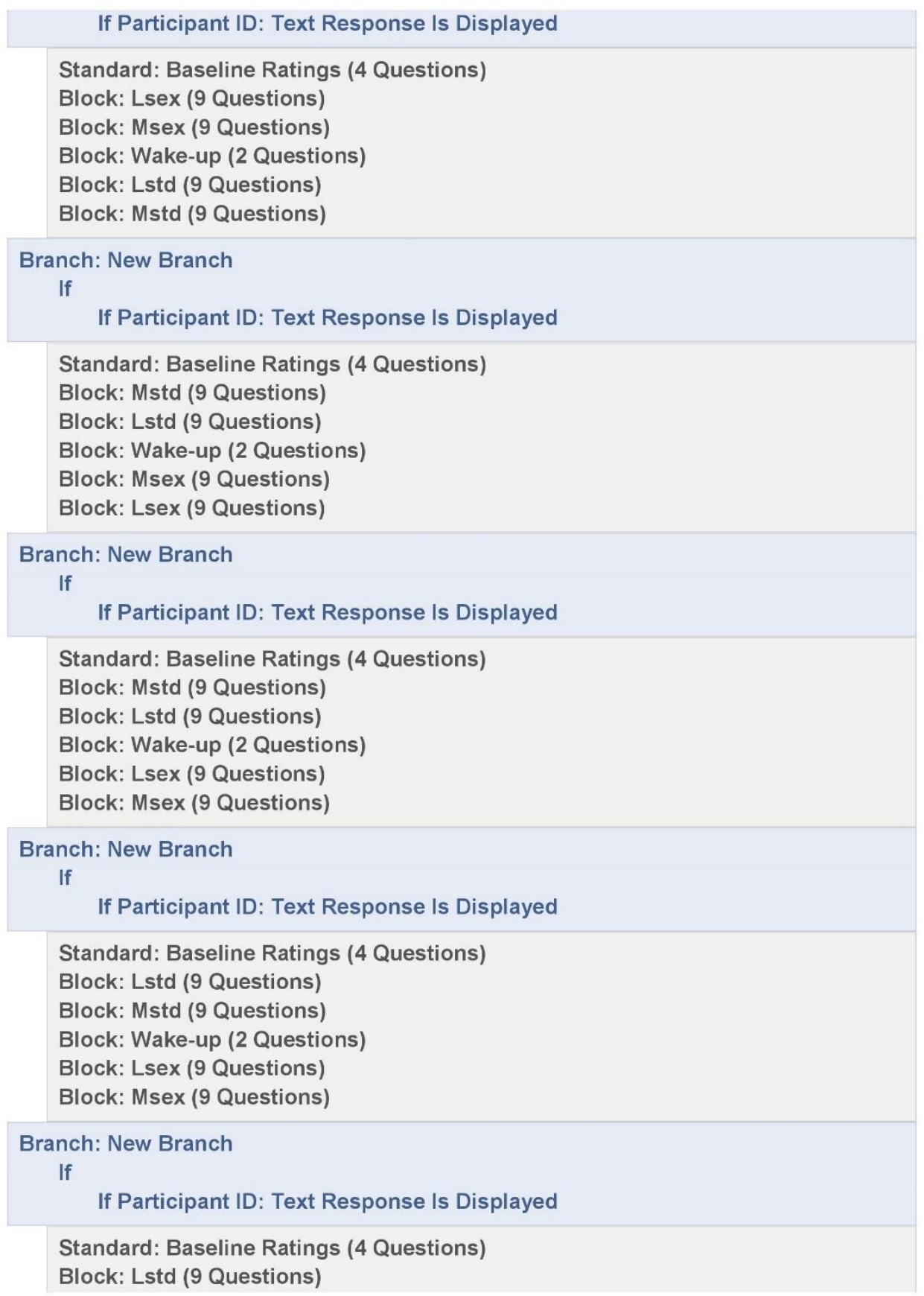


Block: Mstd (9 Questions)

Block: Wake-up (2 Questions)

Block: Msex (9 Questions)

Block: Lsex (9 Questions)

BlockRandomizer: 1 - Evenly Present Elements

Branch: New Branch

If

If Participant ID: Text Response Is Displayed

Block: Pre_preg Reminder Block (2 Questions)

Block: PREGlikely (9 Questions)

Block: Wake-up (2 Questions)

Block: PREGUNlikely (9 Questions)

Branch: New Branch

If

If Participant ID: Text Response Is Displayed

Block: Pre_preg Reminder Block (2 Questions)

Block: PREGUNlikely (9 Questions)

Block: Wake-up (2 Questions)

Block: PREGlikely (9 Questions)

Branch: New Branch

If

If Gender Male Is Selected

Block: Final Questions - Male (13 Questions)

Branch: New Branch

If

If Gender Female Is Selected

Standard: Final Questions - Female (13 Questions)

EndSurvey: Default

Page 4 of 100 
Start of Block: Instructions

ty Thank you for agreeing to take part in this survey on sexual health at the COAT clinic.

Please answer as truthfully and accurately as possible. All responses will be kept confidential.

P_id Participant ID:

date_enrolled Please choose todays date Qualtrics.SurveyEngine.addOnload(function()\{var qid =this.questionld; var calid = qid +'_cal';var y =QBuilder('div'); $\$(y)$. setStyle(\{clear:'both'\});var $\mathrm{d}=$ QBuilder('div',\{className:'yui-skin-sam'\},[QBuilder('div',\{id:calid\}), y ]);var c =this.questionContainer; $\quad c=\$(c)$.down('.QuestionText'); c.appendChild(d);var cal1 =new YAHOO.widget.Calendar(calid); cal1.render();var input $=\$(' Q R \sim '+$ qid);

\$(input). setStyle(\{marginTop:'20px', width:'150px'\});var $p=\$$ (input).up();var $x=Q B u i l d e r(' d i v ')$; $\$(x)$.setStyle(\{clear:'both'\}); p.insert(x,\{position:'before'\});

cal1.selectEvent. subscribe (function(e,dates) $\{$ var date $=$ dates[0][0];if(date[1]

is

consent I consent to the following study

Yes (1)

No (2)

End of Block: Instructions

Start of Block: Demographics

$x \rightarrow$

Page 5 of 100 


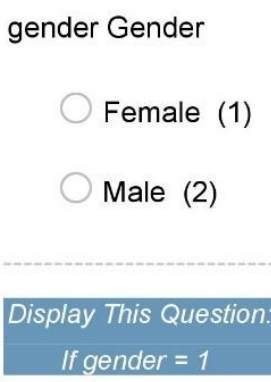




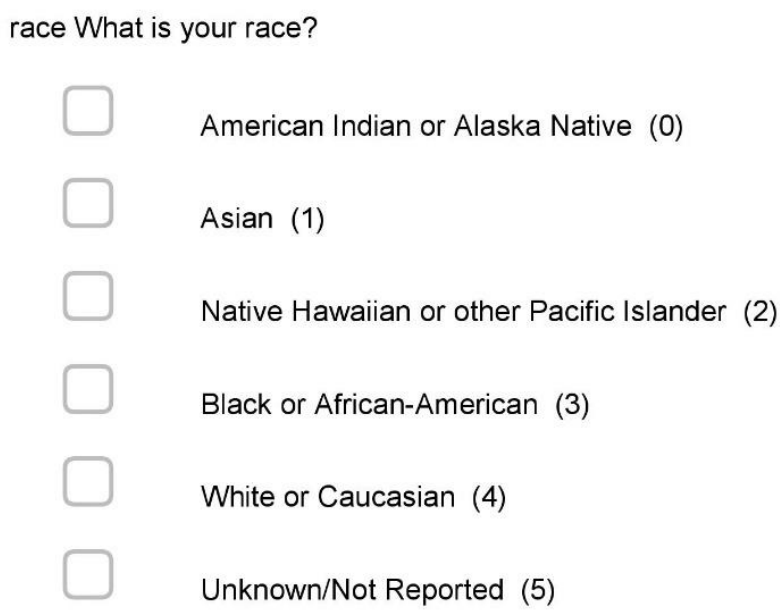


edu Years of education?

(For example, completed high school or GED $=12$ years)

$x \rightarrow$

relationship What is your relationship status?

Never married (1)

Married (2)

Divorced (3)

Separated (4)

Living with a partner (5)

Widowed (6)

$x \rightarrow$

previous_childbirth Do you have any children?

Yes (1)

No (0)

Display This Question:

If previous_childbirth $=1$

numberof_children How many children do you have?

Page 8 of 100 


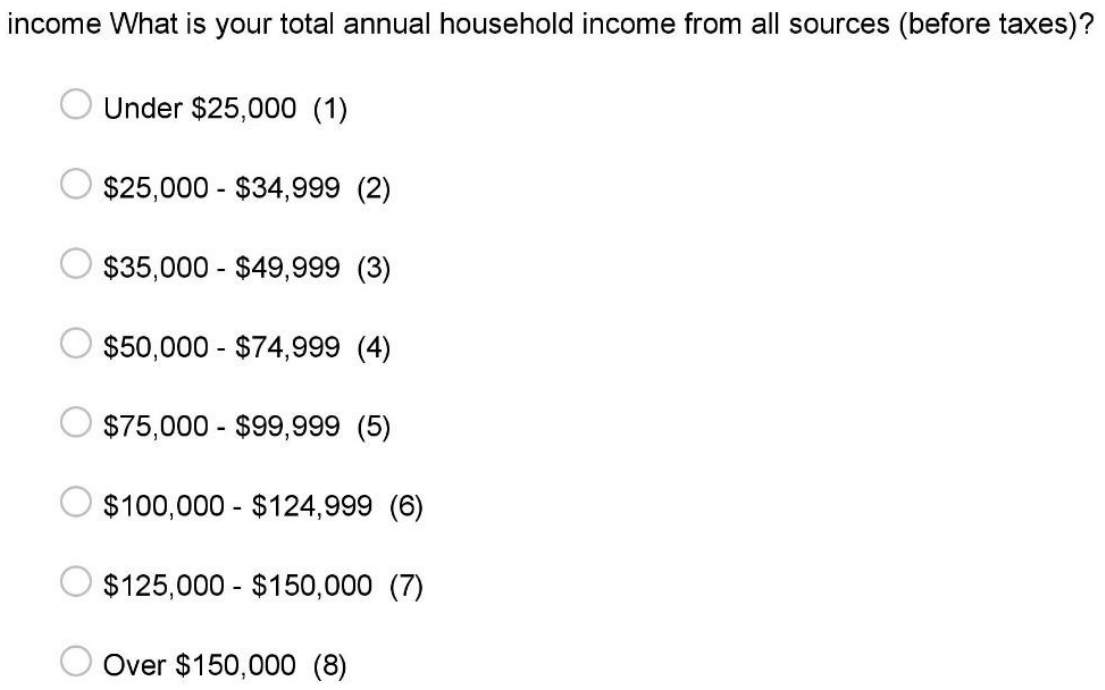


prac_instr

For this task, you will be asked to respond by dragging the cursor along the line. Please note that the numeric response that you provide will appear to the right of each line.

So that you understand how to respond, we have provided the following examples.

NOTE: Please take your time. You will NOT be able to move on to the next example until you provide the correct answer.

水

prac_1 Imagine that a question is "How likely are you to eat dinner tonight?"

Imagine you had a big lunch, and felt that you would definitely not eat dinner tonight (i.e., a $0 \%$ chance that you would eat dinner). Please respond to this question with a $0 \%$ chance you would eat dinner by dragging the cursor to the " 0 ".

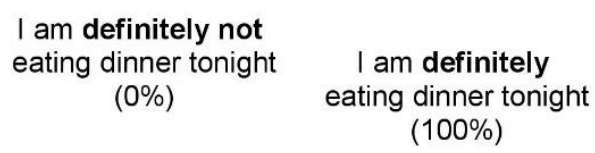

I am definitely eating dinner tonight

$(100 \%)$

$\begin{array}{lllllllllll}0 & 10 & 20 & 30 & 40 & 50 & 60 & 70 & 80 & 90 & 100\end{array}$

How likely are you to eat dinner tonight? ()

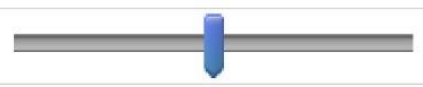




\section{is}

prac_2 Again, imagine that a question is "How likely are you to eat dinner tonight?"

Imagine you had a big lunch, and felt that you almost definitely would not eat dinner tonight (e.g., only a $5 \%$ chance that you would eat dinner). Please respond to this question with a $5 \%$ chance you would eat dinner.

$$
\begin{aligned}
& \text { I am definitely not } \\
& \text { eating dinner tonight I am definitely } \\
& (0 \%) \quad \text { eating dinner tonight } \\
& \text { (100\%) } \\
& \begin{array}{lllllllllll}
0 & 10 & 20 & 30 & 40 & 50 & 60 & 70 & 80 & 90 & 100
\end{array}
\end{aligned}
$$

How likely are you to eat dinner tonight? ()

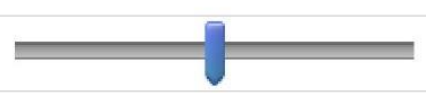


prac_3 Again, imagine that a question is "How likely are you to eat dinner tonight?"

Imagine you had a normal-sized lunch, and felt that you probably would not eat dinner tonight (e.g., a $25 \%$ chance that you would eat dinner). Please respond to this question with a $25 \%$ chance you would eat dinner.

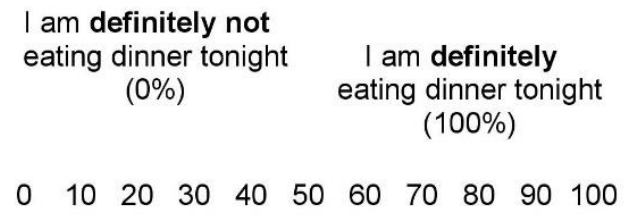

How likely are you to eat dinner tonight? ()

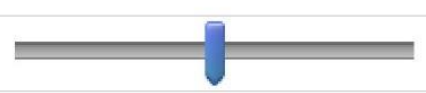


is

prac_4 Again, imagine that a question is "How likely are you to eat dinner tonight?"

Imagine you had a normal-sized lunch, and felt that you were unsure if you would eat dinner tonight (e.g., a $50 \%$ chance that you would eat dinner). Please respond to this question with a $50 \%$ chance you would eat dinner.

$$
\begin{aligned}
& \text { I am definitely not } \\
& \text { eating dinner tonight I am definitely } \\
& (0 \%) \quad \text { eating dinner tonight } \\
& \text { (100\%) } \\
& \begin{array}{lllllllllll}
0 & 10 & 20 & 30 & 40 & 50 & 60 & 70 & 80 & 90 & 100
\end{array}
\end{aligned}
$$

How likely are you to eat dinner tonight? ()

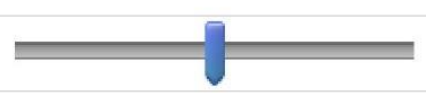


prac_5 Again, imagine that a question is "How likely are you to eat dinner tonight?"

Imagine you had a smaller-sized lunch, and felt that you would most likely eat dinner tonight (e.g., a $75 \%$ chance that you would eat dinner). Please respond to this question with a $75 \%$ chance you would eat dinner.

$$
\begin{aligned}
& \text { I am definitely not } \\
& \text { eating dinner tonight I am definitely } \\
& (0 \%) \quad \text { eating dinner tonight } \\
& \text { (100\%) } \\
& \begin{array}{lllllllllll}
0 & 10 & 20 & 30 & 40 & 50 & 60 & 70 & 80 & 90 & 100
\end{array}
\end{aligned}
$$

How likely are you to eat dinner tonight? ()

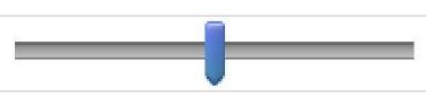


*⿻一𠃋十

prac_5 Again, imagine that a question is "How likely are you to eat dinner tonight?"

Imagine you had a smaller-sized or no lunch at all, and felt that you were extremely likely to eat dinner tonight (e.g., a 95\% chance that you would eat dinner). Please respond to this question with a $95 \%$ chance you would eat dinner.

$$
\begin{aligned}
& \text { I am definitely not } \\
& \text { eating dinner tonight I am definitely } \\
& (0 \%) \quad \text { eating dinner tonight } \\
& \text { (100\%) } \\
& \begin{array}{lllllllllll}
0 & 10 & 20 & 30 & 40 & 50 & 60 & 70 & 80 & 90 & 100
\end{array}
\end{aligned}
$$

How likely are you to eat dinner tonight? ()

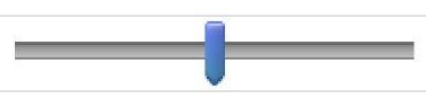




\section{is}

prac_6 Again, imagine that a question is "How likely are you to eat dinner tonight?"

Imagine you hadn't eaten all day, and felt that you would definitely eat dinner tonight (i.e., a $100 \%$ chance that you would eat dinner). Please respond to this question with a $100 \%$ chance you would eat dinner.

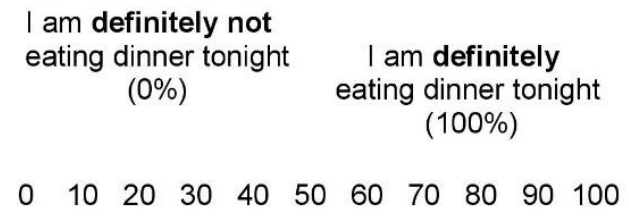

How likely are you to eat dinner tonight? ()

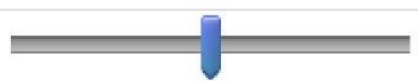


exmplnote

Please note that these are just examples.

You can mark anywhere on the response line, not just at the marks as shown in these examples.

\section{End of Block: Dinner Practice}

Start of Block: 10\$ Monetary Discounting Task - Patrick and Johnson 2015 - JAEB Free Condition

intro_mddx_10 For each of the next 16 questions, imagine the following hypothetical (pretend) scenario:

You are presented with a choice between money now or later.

For the money now option: The money is deposited automatically into your bank account now and you are immediately free to pursue other activities.

For the money later option: You don't have to wait at the computer or return to the computer to have the (money deposited into your bank account). Instead, you are immediately free to pursue other activities. After the specified time, the (money is deposited automatically into your bank account), regardless of where you are at that time.

Your job is to use the slider tool to tell us the amount of money that you would like to receive immediately that would make you feel JUST AS GOOD as you would if you were to receive money after the specified time.

Although the scenarios are pretend, we ask that you consider each scenario as if it was real and as if it was the only scenario you would face today.

Finally, when considering each scenario, you should take into account your financial circumstances (e.g., current account balance, rent or bills due). You will now take a short quiz to confirm your understanding of these instructions. 


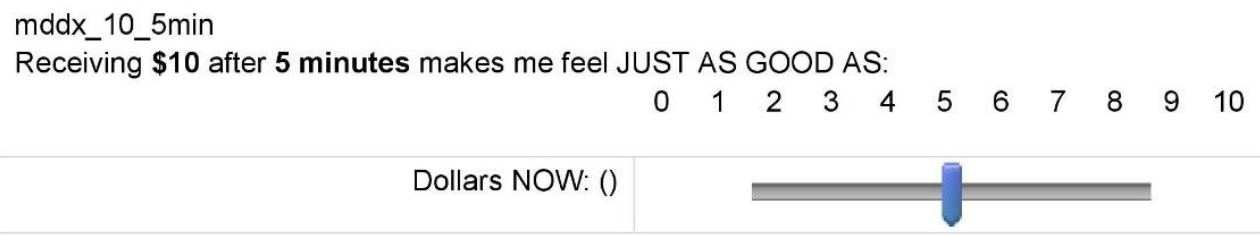

Page Break

Page 22 of 100 


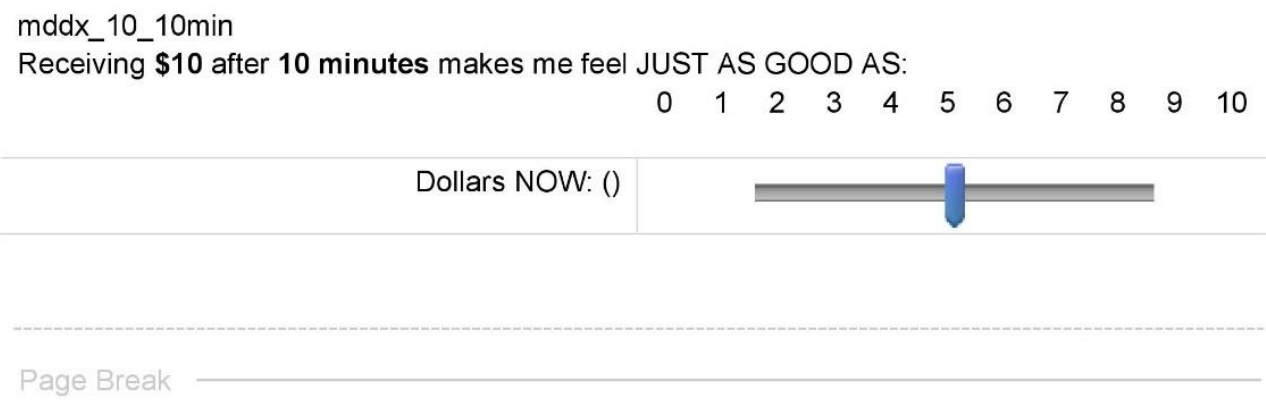




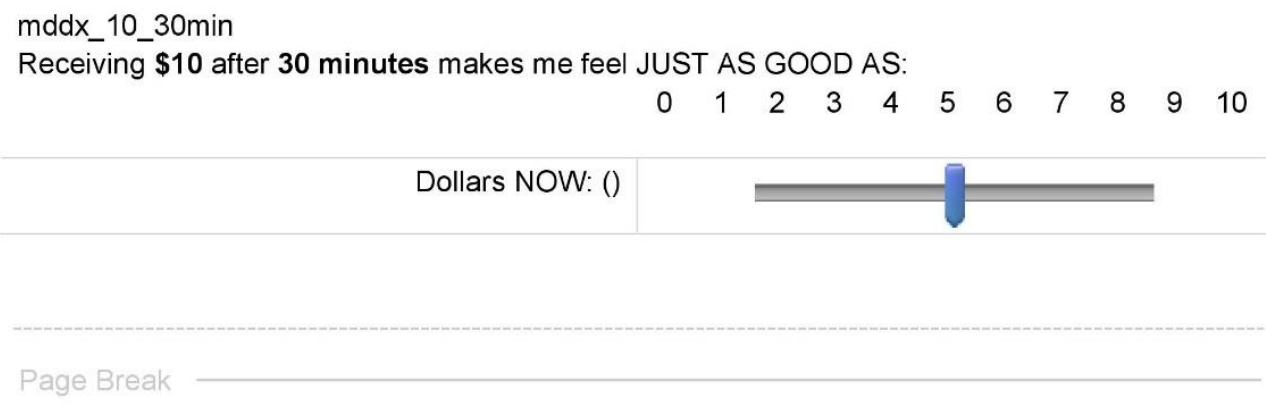

Page 24 of 100 


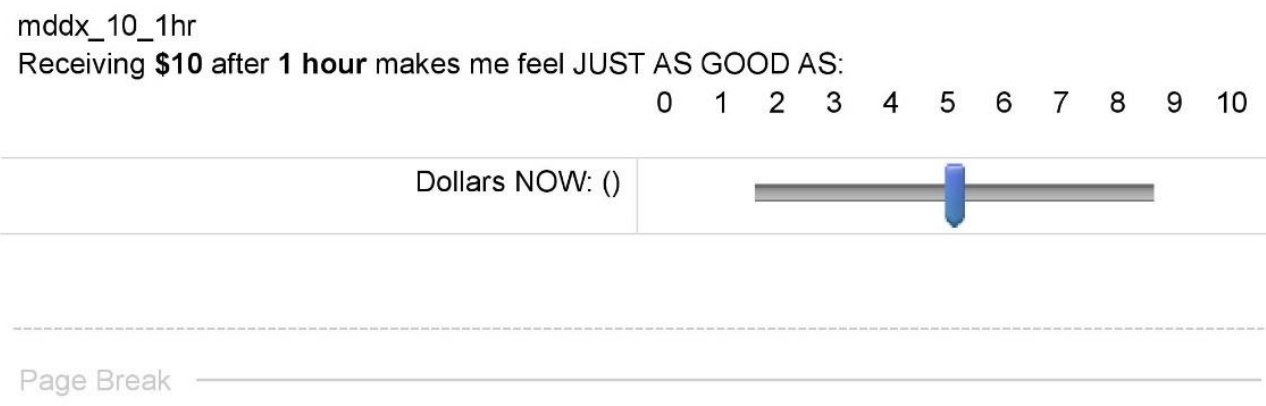

Page 25 of 100 


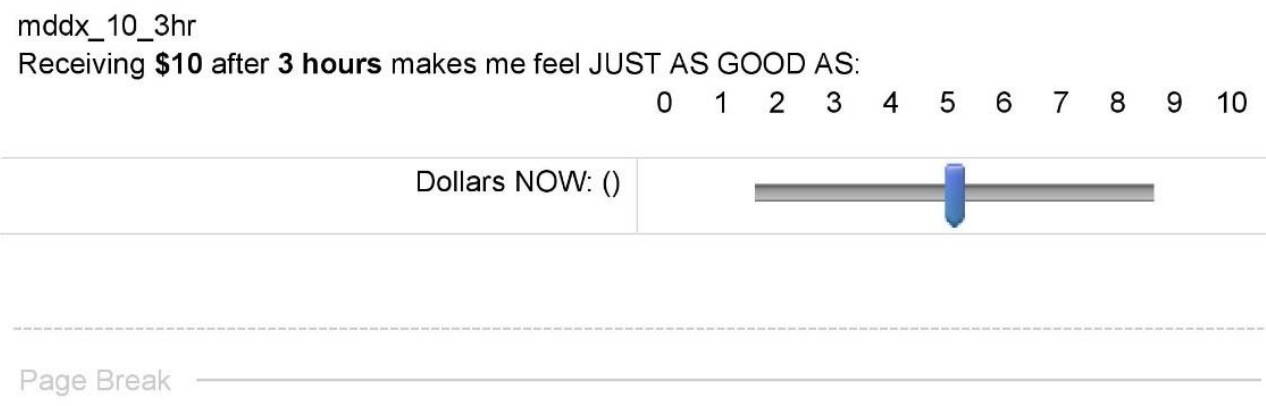

Page 26 of 100 


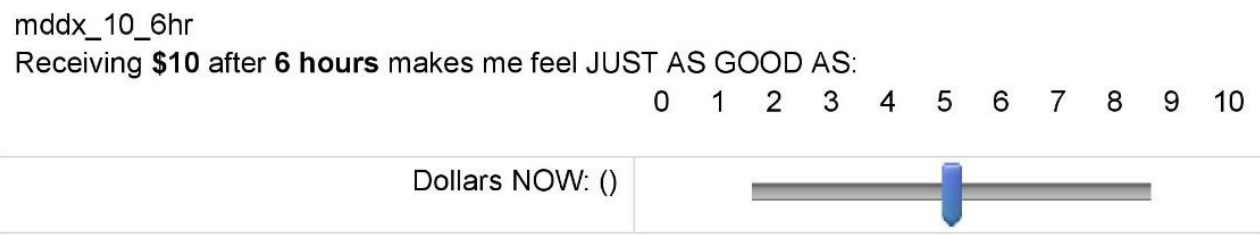

Page Break

Page 27 of 100 
mddx_10_12hr

Receiving $\$ 10$ after 12 hours makes me feel JUST AS GOOD AS:

$\begin{array}{lllllllllll}0 & 1 & 2 & 3 & 4 & 5 & 6 & 7 & 8 & 9 & 10\end{array}$

Dollars NOW: ()

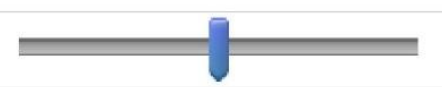

Page Break

Page 28 of 100 


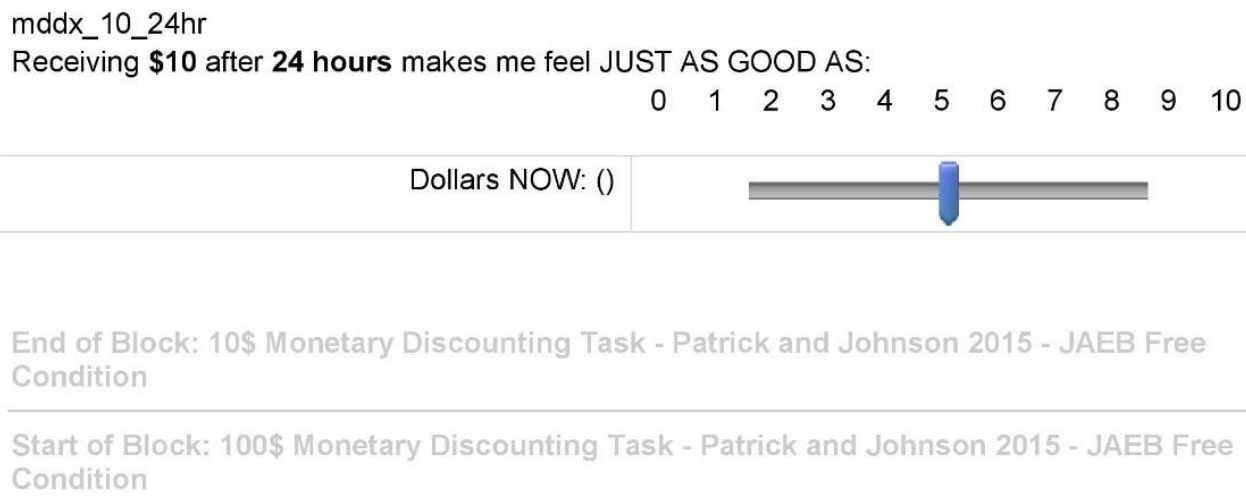


intro_mddx_100 For each of the next 16 questions, imagine the following hypothetical (pretend) scenario:

You are presented with a choice between money now or later.

For the money now option: The money is deposited automatically into your bank account now and you are immediately free to pursue other activities.

For the money later option: You don't have to wait at the computer or return to the computer to have the (money deposited into your bank account). Instead, you are immediately free to pursue other activities. After the specified time, the (money is deposited automatically into your bank account), regardless of where you are at that time.

Your job is to use the slider tool to tell us the amount of money that you would like to receive immediately that would make you feel JUST AS GOOD as you would if you were to receive money after the specified time.

Although the scenarios are pretend, we ask that you consider each scenario as if it was real and as if it was the only scenario you would face today.

Finally, when considering each scenario, you should take into account your financial circumstances (e.g., current account balance, rent or bills due). You will now take a short quiz to confirm your understanding of these instructions.

mddx_100_5min

Receiving $\$ 100$ after 5 minutes makes me feel JUST AS GOOD AS:

$\begin{array}{lllllllllll}0 & 10 & 20 & 30 & 40 & 50 & 60 & 70 & 80 & 90 & 100\end{array}$

Dollars NOW: ()

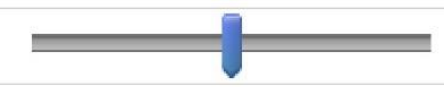

Page 30 of 100 


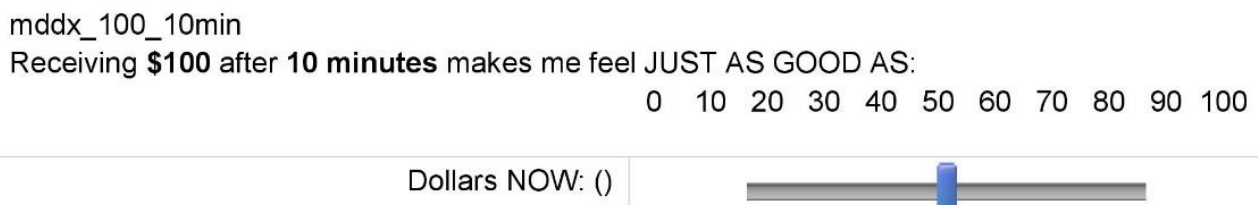




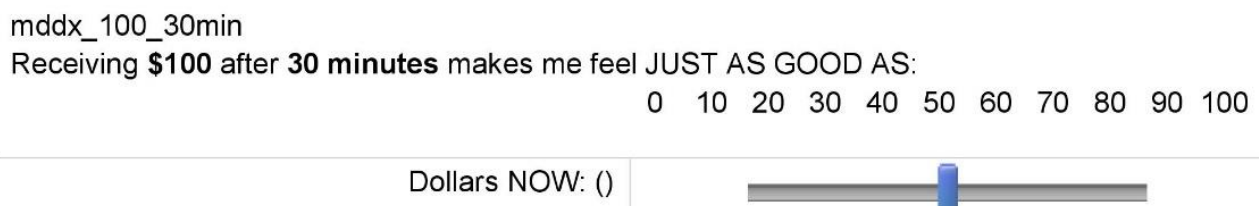




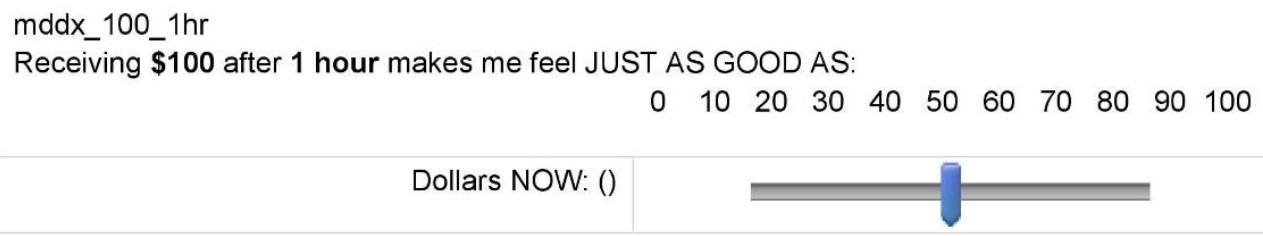

Page Break

Page 33 of 100 


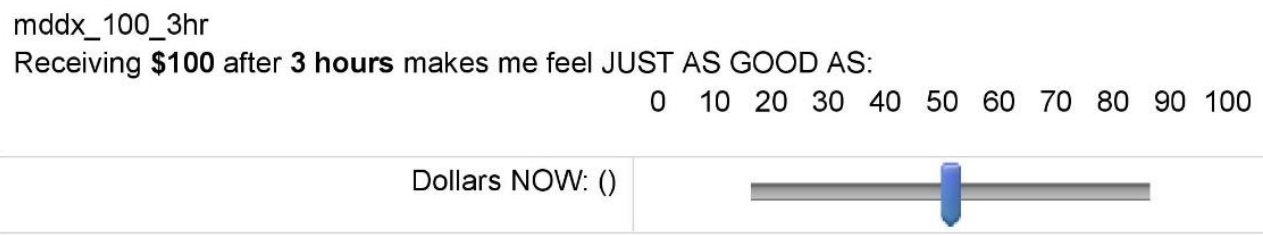

Page Break

Page 34 of 100 


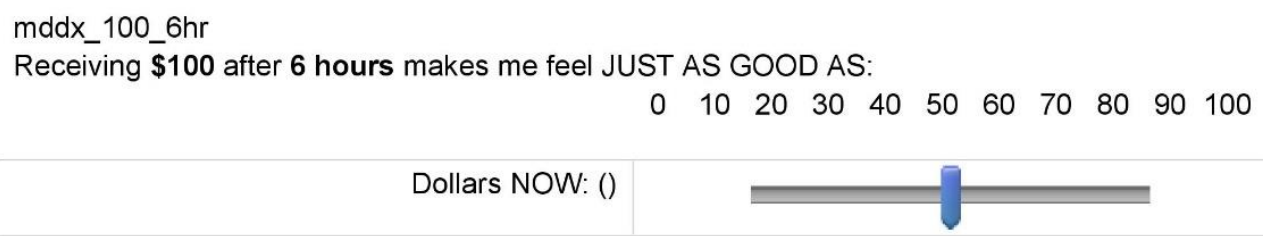

Page Break

Page 35 of 100 
mddx_100_12hr

Receiving \$100 after 12 hours makes me feel JUST AS GOOD AS:

$\begin{array}{lllllllllll}0 & 10 & 20 & 30 & 40 & 50 & 60 & 70 & 80 & 90 & 100\end{array}$

Dollars NOW: ()

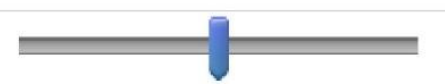

Page Break

Page 36 of 100 
mddx_100_24hr

Receiving $\$ 100$ after $\mathbf{2 4}$ hours makes me feel JUST AS GOOD AS:

$\begin{array}{lllllllllll}0 & 10 & 20 & 30 & 40 & 50 & 60 & 70 & 80 & 90 & 100\end{array}$

Dollars NOW: ()

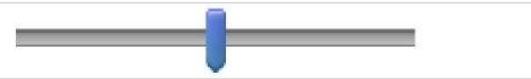

End of Block: 100\$ Monetary Discounting Task - Patrick and Johnson 2015 - JAEB Free Condition

Start of Block: Baseline Ratings

appearance_intro2

For the next task, we will ask you hypothetical (pretend) questions about your willingness to have sex in various situations

For the purpose of this task, please pretend that you are NOT currently in a committed sexual relationship even if you are.

In other words, please pretend that you are single and available, and that you are not cheating on anybody if you indicate you would have sex with somebody in this task.

appearance_intro1 For each person you think about, we would like you to think about how attractive that person is based on physical appearance alone.

Please think about whether each person is someone that you would consider having sex with in the right environment and if you liked the person's personality.

Page Break

Page 37 of 100 


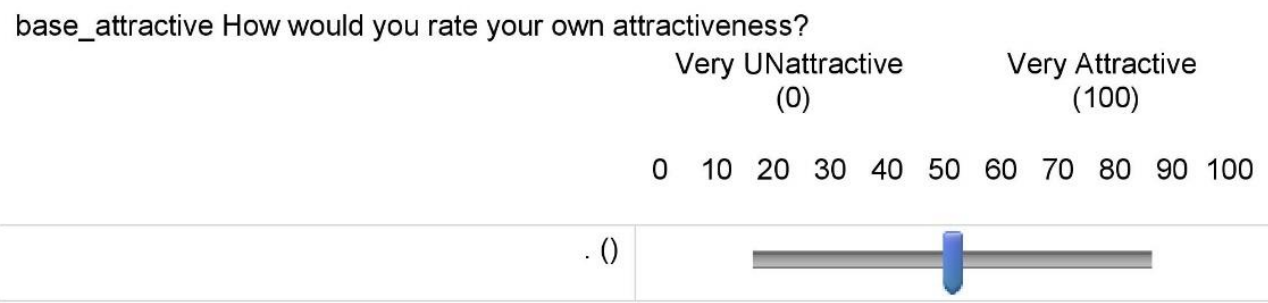

base_sexdesire

How interested are you in having sex right now?

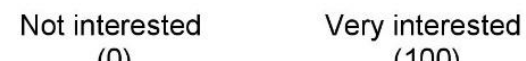

(0)

(100)

$\begin{array}{lllllllllll}0 & 10 & 20 & 30 & 40 & 50 & 60 & 70 & 80 & 90 & 100\end{array}$

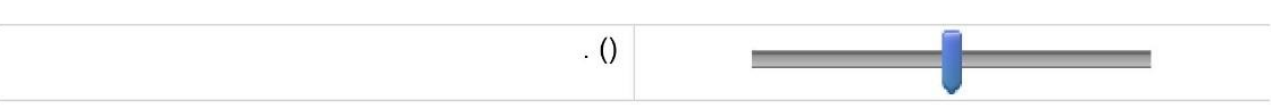

\section{End of Block: Baseline Ratings}

Start of Block: Msex

msex_attract

Imagine the person you would MOST want to have sex with.

You are very interested in having sex with this individual.

How would you rate this individual's attractiveness?

$\begin{array}{cc}\text { Very UNattractive } & \text { Very Attractive } \\ (0) & (100)\end{array}$

$\begin{array}{lllllllllll}0 & 10 & 20 & 30 & 40 & 50 & 60 & 70 & 80 & 90 & 100\end{array}$

. ()

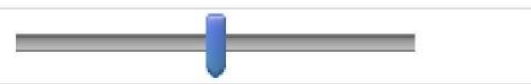

Page 38 of 100 
msex_0

Imagine the person you would MOST want to have sex with.

You are very interested in having sex with this individual.

Imagine that you have just met this person. You are getting along great and they are interested in having sex with you now.

Imagine you are confident that there is no chance of pregnancy, for example, you know that one of you is either on the pill, has had their "tubes tied", or had a vasectomy.

Imagine that there is a condom readily and immediately available.

Please rate how likely you are to use a condom or not.

I will definitely have sex I will definitely have sex with this person with this person

right away

without a condom.

(0)

right away

with a condom.

(100)

$\begin{array}{lllllllllll}0 & 10 & 20 & 30 & 40 & 50 & 60 & 70 & 80 & 90 & 100\end{array}$

()

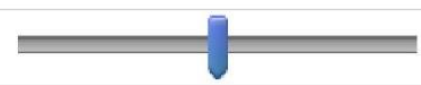

Page Break

Page 40 of 100 


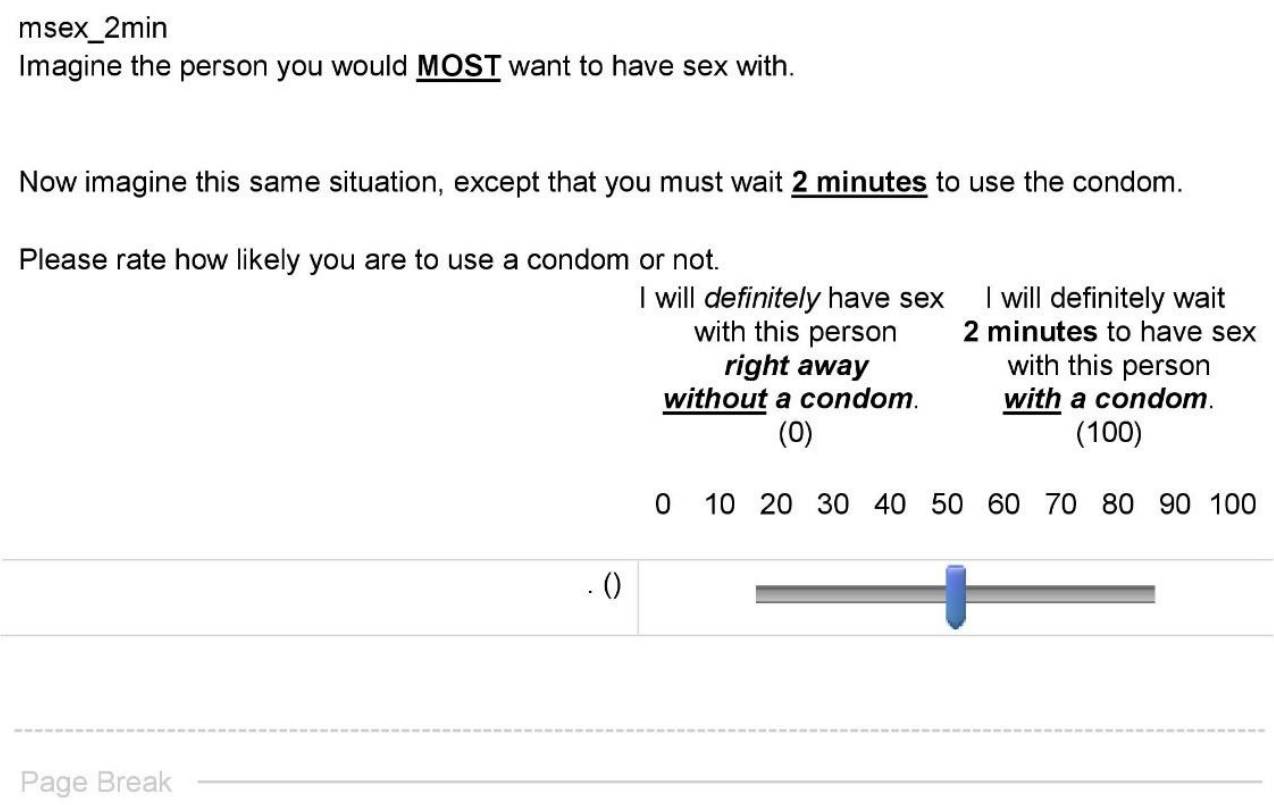




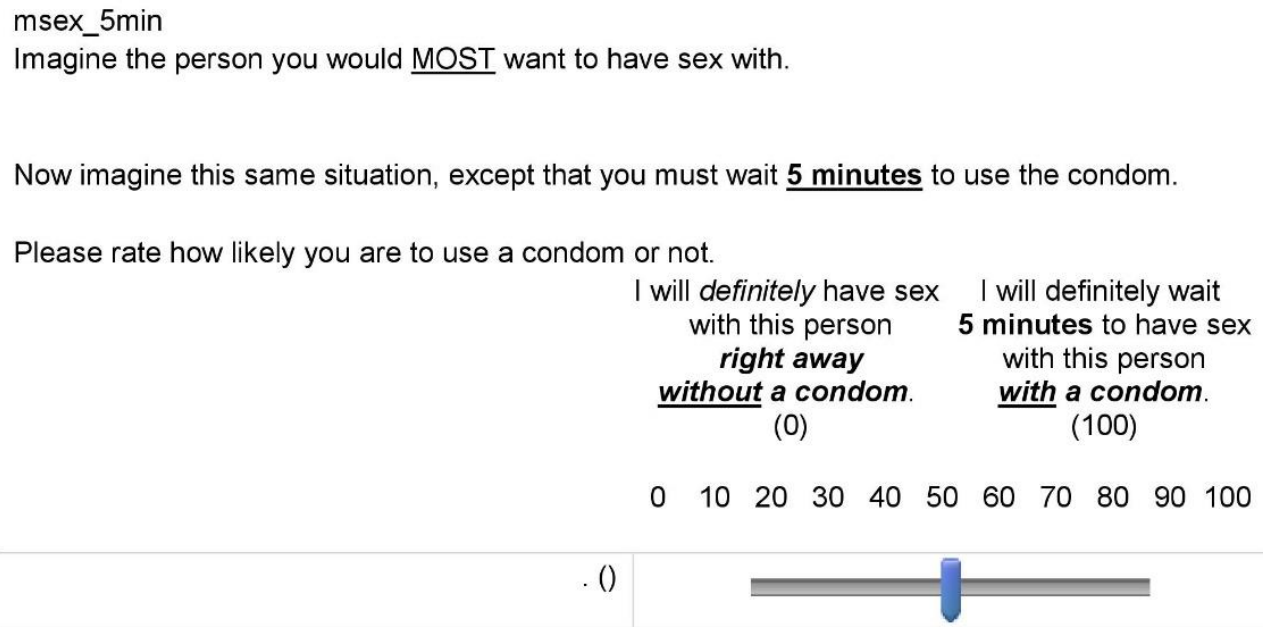


msex_15min

Imagine the person you would MOST want to have sex with.

Now imagine this same situation, except that you must wait 15 minutes to use the condom.

Please rate how likely you are to use a condom or not.

I will definitely have sex with this person right away without a condom (0)

I will definitely wait 15 minutes to have sex with this person with a condom.

(100)

$\begin{array}{lllllllllll}0 & 10 & 20 & 30 & 40 & 50 & 60 & 70 & 80 & 90 & 100\end{array}$ ()

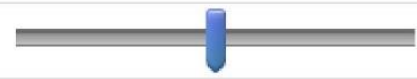




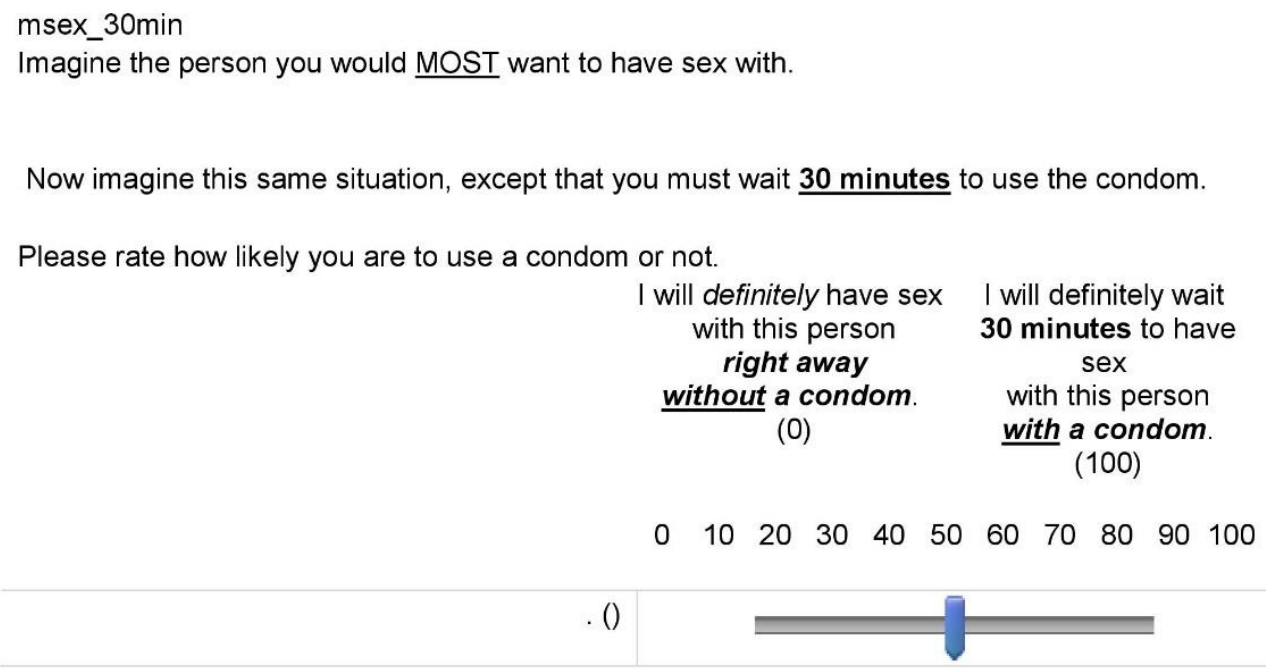


msex_1hr
Imagine the person you would MOST want to have sex with.

Now imagine this same situation, except that you must wait 1 hour to use the condom.

Please rate how likely you are to use a condom or not.

I will definitely have sex I will definitely wait with this person $\quad 1$ hour to have sex right away with this person without a condom. with a condom.

(0) (100)

$\begin{array}{lllllllllll}0 & 10 & 20 & 30 & 40 & 50 & 60 & 70 & 80 & 90 & 100\end{array}$

()

$\underline{\underline{2}}$

Page Break

Page 45 of 100 
msex_3hr
Imagine the person you would MOST want to have sex with.

Now imagine this same situation, except that you must wait $\underline{3 \text { hours }}$ to use the condom.

Please rate how likely you are to use a condom or not.

I will definitely have sex I will definitely wait with this person 3 hours to have sex right away with this person without a condom. with a condom.

(0) (100)

$\begin{array}{lllllllllll}0 & 10 & 20 & 30 & 40 & 50 & 60 & 70 & 80 & 90 & 100\end{array}$

()

$\underline{\overline{2}}$

Page Break

Page 46 of 100 
msex_6hr
Imagine the person you would MOST want to have sex with.

Now imagine this same situation, except that you must wait $\underline{6 \text { hours }}$ to use the condom.

Please rate how likely you are to use a condom or not.

I will definitely have sex I will definitely wait

with this person $\quad 6$ hours to have sex

right away with this person

without a condom. with a condom.

(0)

(100)

$\begin{array}{lllllllllll}0 & 10 & 20 & 30 & 40 & 50 & 60 & 70 & 80 & 90 & 100\end{array}$

()

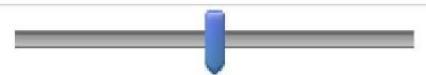

End of Block: Msex

Start of Block: Lsex

Isex_attract

NOW, Imagine the person you would LEAST want to have sex with.

You are interested in having sex with this individual, just barely though.

How would you rate this individual's attractiveness?

Very UNattractive Very Attractive

(0)

(100)

$\begin{array}{lllllllllll}0 & 10 & 20 & 30 & 40 & 50 & 60 & 70 & 80 & 90 & 100\end{array}$

Page Break

Page 47 of 100 


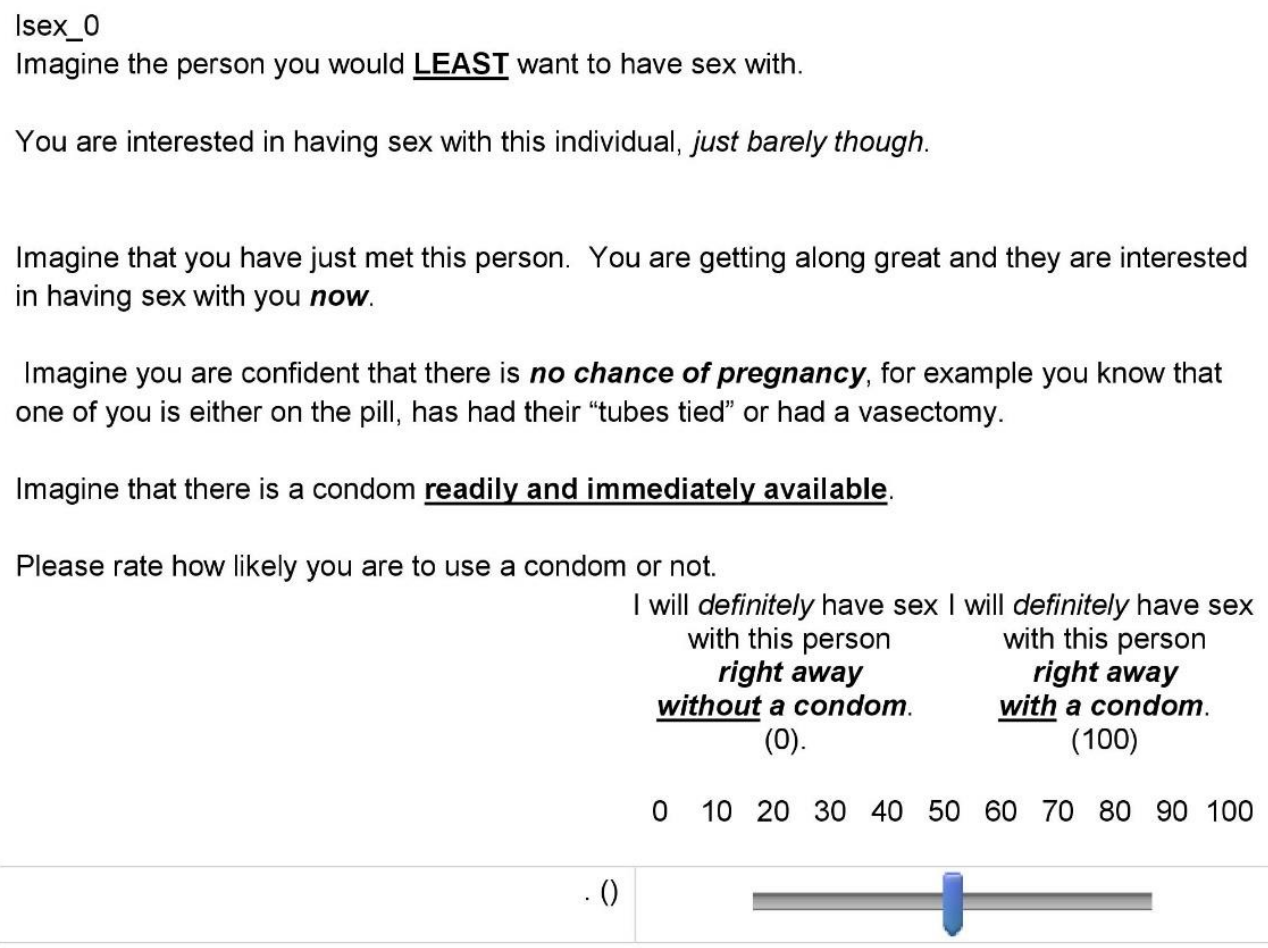


Isex_2min
Imagine the person you would LEAST want to have sex with.

Now imagine this same situation, except that you must wait $\underline{2}$ minutes to use the condom.

Please rate how likely you are to use a condom or not.

I will definitely have sex I will definitely wait with this person 2 minutes to have sex right away with this person without a condom. with a condom.

(0) (100)

$\begin{array}{lllllllllll}0 & 10 & 20 & 30 & 40 & 50 & 60 & 70 & 80 & 90 & 100\end{array}$

()

$\underline{\underline{2}}$

Page Break

Page 49 of 100 
Isex_5min

Imagine the person you would LEAST want to have sex with.

Now imagine this same situation, except that you must wait $\underline{5 \text { minutes }}$ to use the condom.

Please rate how likely you are to use a condom or not.

I will definitely have sex I will definitely wait with this person 5 minutes to have sex right away with this person without a condom. with a condom.

(0) (100)

$\begin{array}{lllllllllll}0 & 10 & 20 & 30 & 40 & 50 & 60 & 70 & 80 & 90 & 100\end{array}$

()

$\underline{\underline{2}}$

Page Break

Page 50 of 100 
Isex_15min
Imagine the person you would LEAST want to have sex with.

Now imagine this same situation, except that you must wait $\underline{15 \text { minutes }}$ to use the condom.

Please rate how likely you are to use a condom or not.

I will definitely have sex with this person right away without a condom. (0)

I will definitely wait 15 minutes to have sex with this person with a condom.

(100)

$\begin{array}{lllllllllll}0 & 10 & 20 & 30 & 40 & 50 & 60 & 70 & 80 & 90 & 100\end{array}$ ()

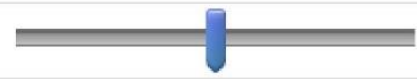


Isex_30min

Imagine the person you would LEAST want to have sex with.

Now imagine this same situation, except that you must wait $\underline{30 \text { minutes }}$ to use the condom.

Please rate how likely you are to use a condom or not.

I will definitely have sex I will definitely wait with this person $\quad \mathbf{3 0}$ minutes to have right away sex without a condom. (0) with this person with a condom.

(100)

$\begin{array}{lllllllllll}0 & 10 & 20 & 30 & 40 & 50 & 60 & 70 & 80 & 90 & 100\end{array}$ ()

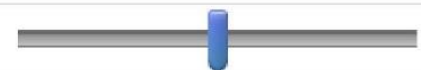


Isex_1hr
Imagine the person you would LEAST want to have sex with.

Now imagine this same situation, except that you must wait 1 hour to use the condom.

Please rate how likely you are to use a condom or not.

I will definitely have sex I will definitely wait with this person $\quad 1$ hour to have sex right away with this person without a condom. with a condom.

(0) (100)

$\begin{array}{lllllllllll}0 & 10 & 20 & 30 & 40 & 50 & 60 & 70 & 80 & 90 & 100\end{array}$

()

$\underline{\overline{2}}$

Page Break

Page 53 of 100 
Isex_3hr

Imagine the person you would LEAST want to have sex with.

Now imagine this same situation, except that you must wait $\underline{3 \text { hours }}$ to use the condom.

Please rate how likely you are to use a condom or not.

I will definitely have sex I will definitely wait with this person $\quad 3$ hours to have sex right away with this person without a condom. with a condom.

(0) (100)

$\begin{array}{lllllllllll}0 & 10 & 20 & 30 & 40 & 50 & 60 & 70 & 80 & 90 & 100\end{array}$

()

$\underline{\overline{2}}$

Page Break

Page 54 of 100 

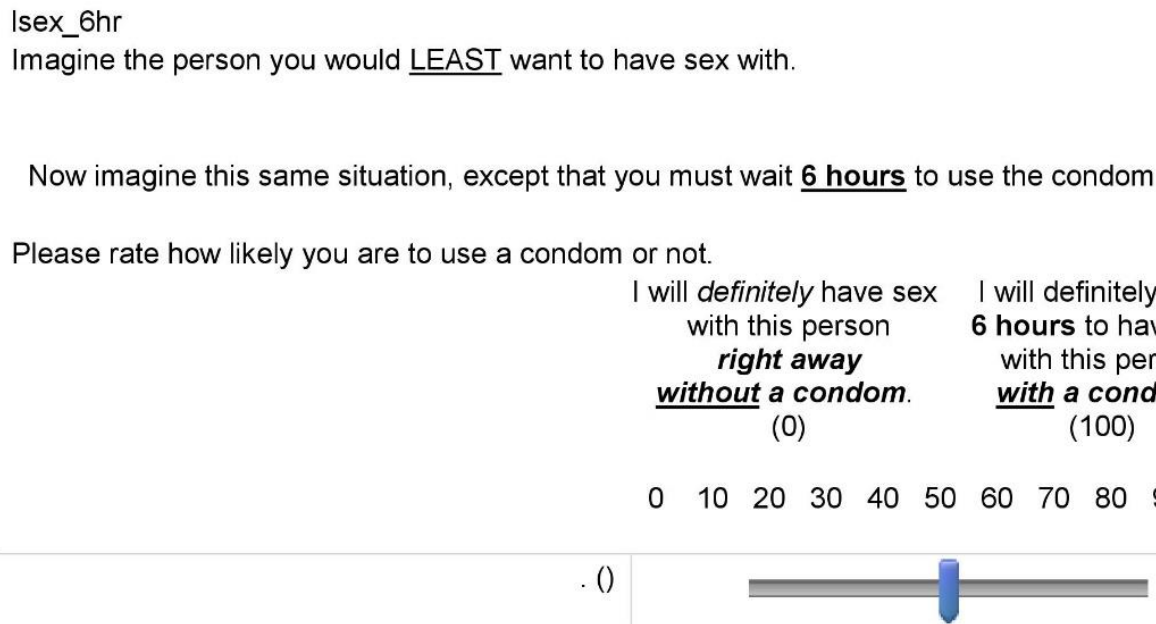

End of Block: Lsex

Start of Block: Wake-up

*

wakeup1 Please enter the text below:

West Virginia

*

wakeup3 Please move the slider to 75

$\begin{array}{lllllllllll}0 & 10 & 20 & 30 & 40 & 50 & 60 & 70 & 80 & 90 & 100\end{array}$

Slide me to 75 ()

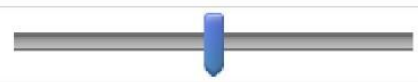




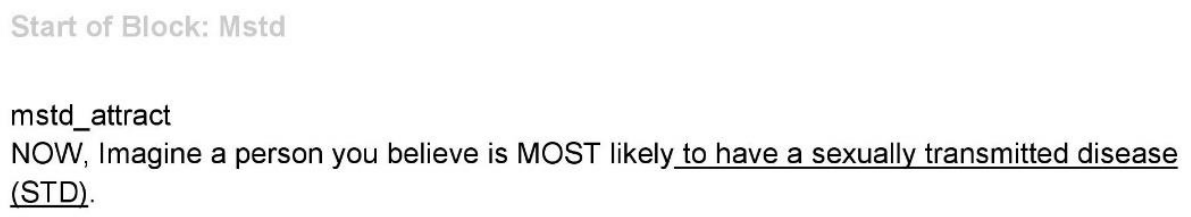

$\begin{array}{lllllllllll}0 & 10 & 20 & 30 & 40 & 50 & 60 & 70 & 80 & 90 & 100\end{array}$

()

$\underline{\underline{2}}$


mstd_0

Imagine a person you believe is MOST likely to have a sexually transmitted disease (STD).

Imagine that you have just met this person. You are getting along great and they are interested in having sex with you now.

Imagine you are confident that there is no chance of pregnancy, for example you know that one of you is either on the pill, has had their "tubes tied" or had a vasectomy.

Imagine that there is a condom readily and immediately available.

Please rate how likely you are to use a condom or not.

I will definitely have sex I will definitely have sex with this person with this person

right away right away

without a condom.

(0)

with a condom.

(100)

$\begin{array}{lllllllllll}0 & 10 & 20 & 30 & 40 & 50 & 60 & 70 & 80 & 90 & 100\end{array}$

()

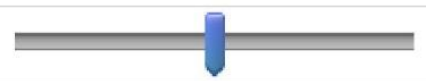

Page Break

Page 57 of 100 


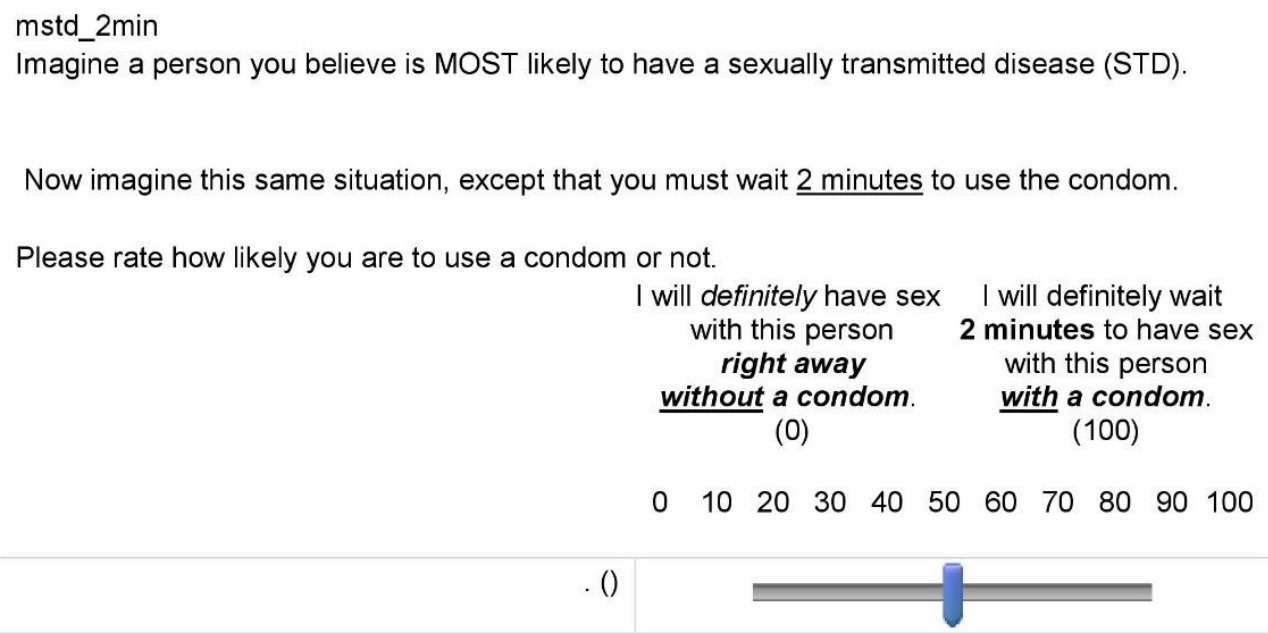

Page Break

Page 58 of 100 


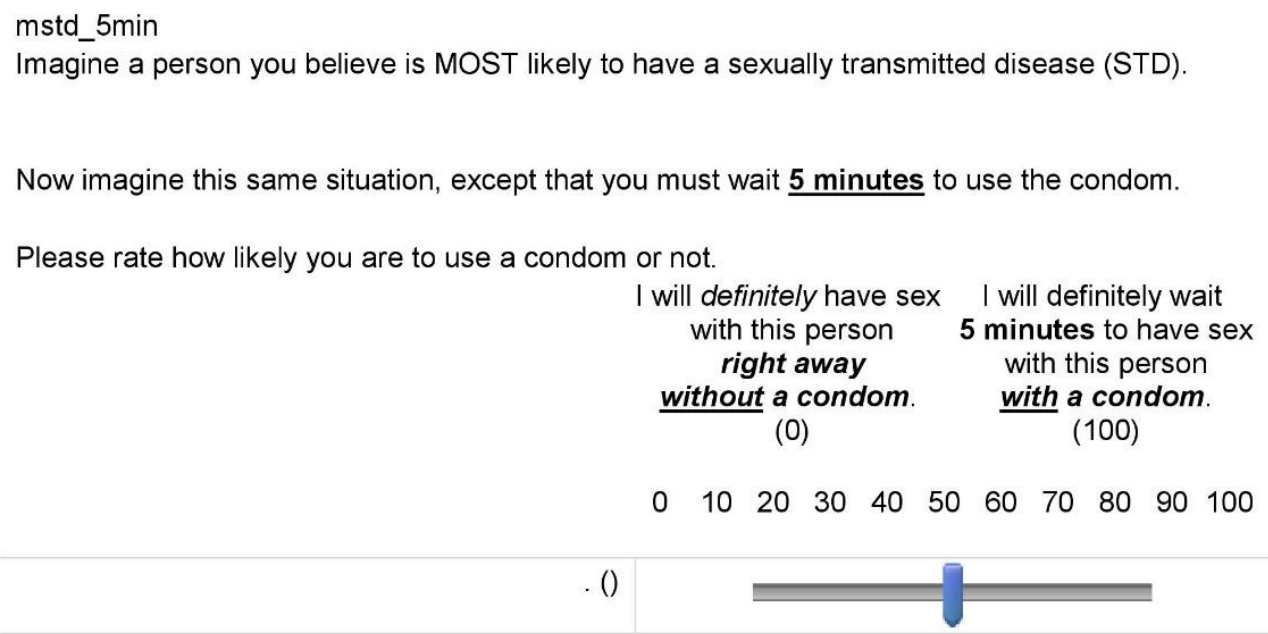


mstd_15min

Imagine a person you believe is MOST likely to have a sexually transmitted disease (STD).

Now imagine this same situation, except that you must wait $\underline{15 \text { minutes }}$ to use the condom.

Please rate how likely you are to use a condom or not.

I will definitely have sex with this person right away without a condom

I will definitely wait 15 minutes to have sex with this person with a condom.

$\begin{array}{lllllllllll}0 & 10 & 20 & 30 & 40 & 50 & 60 & 70 & 80 & 90 & 100\end{array}$ ()

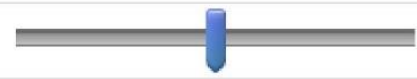




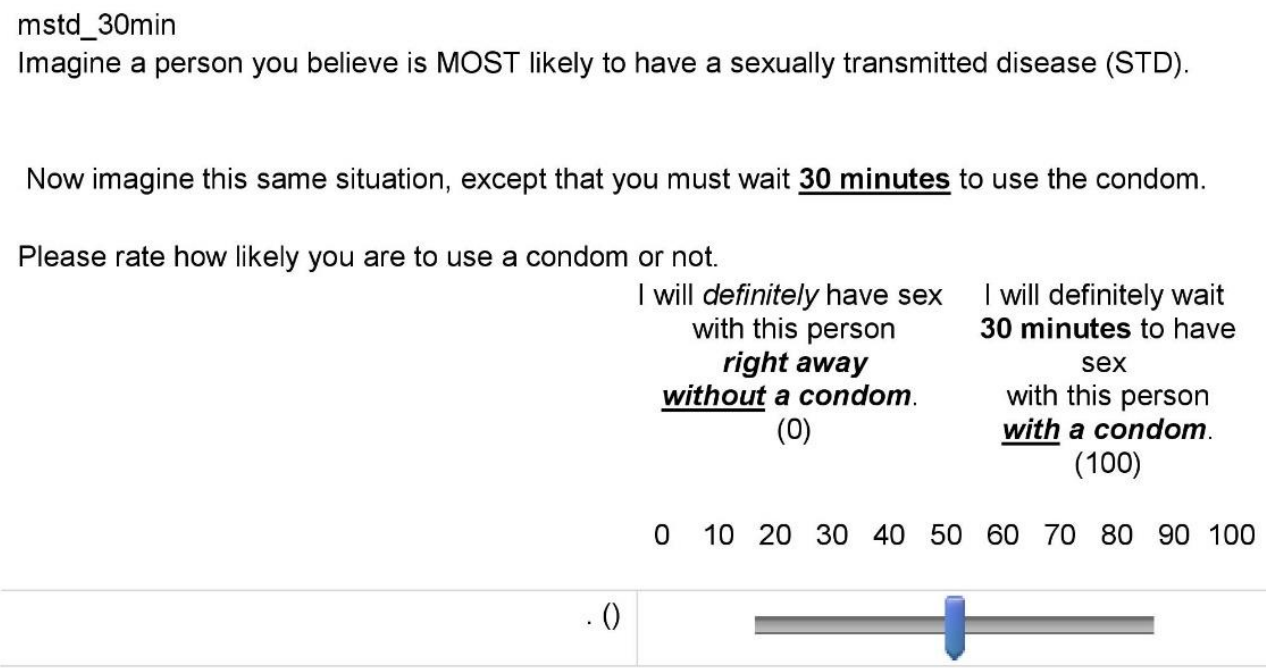




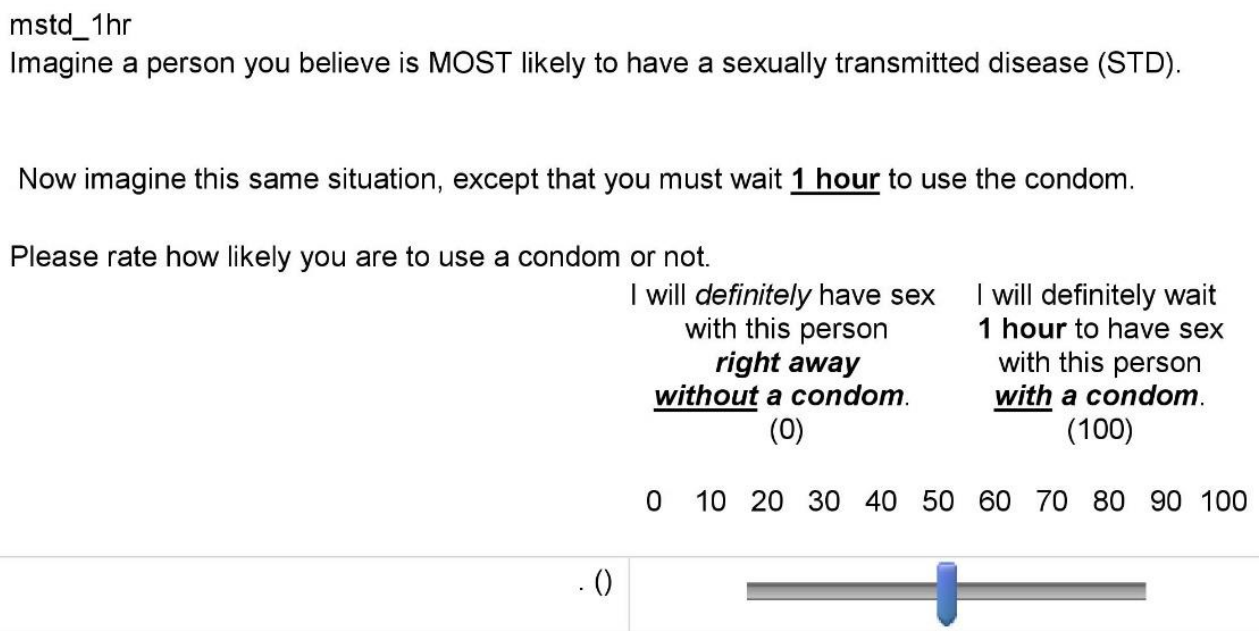




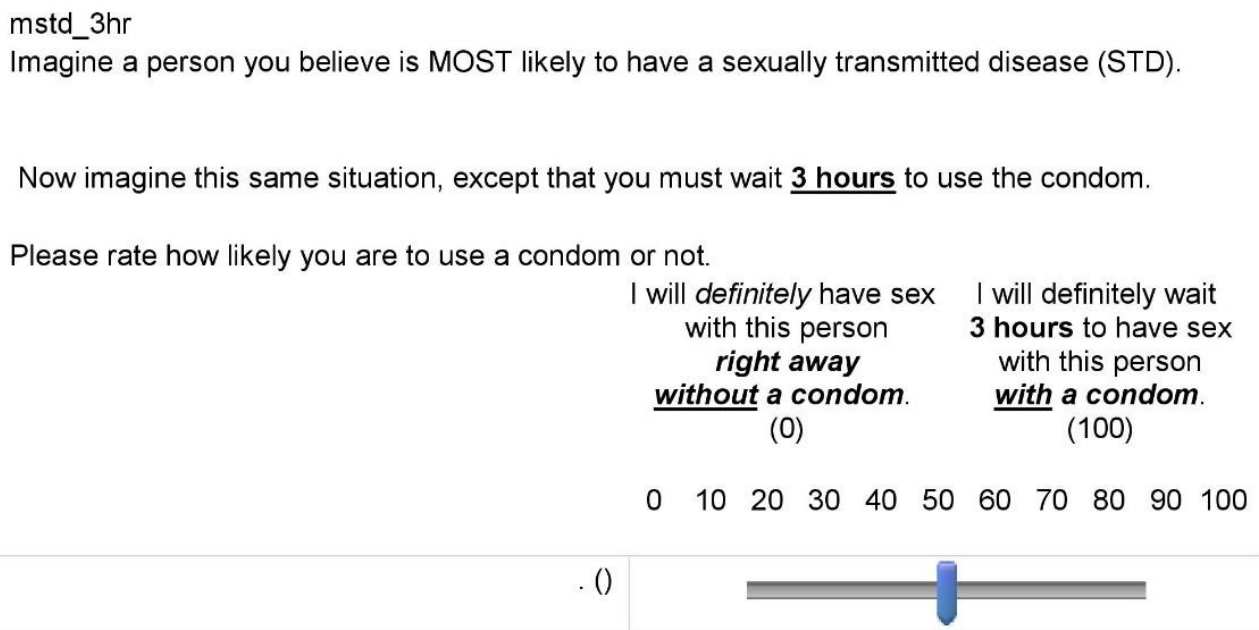




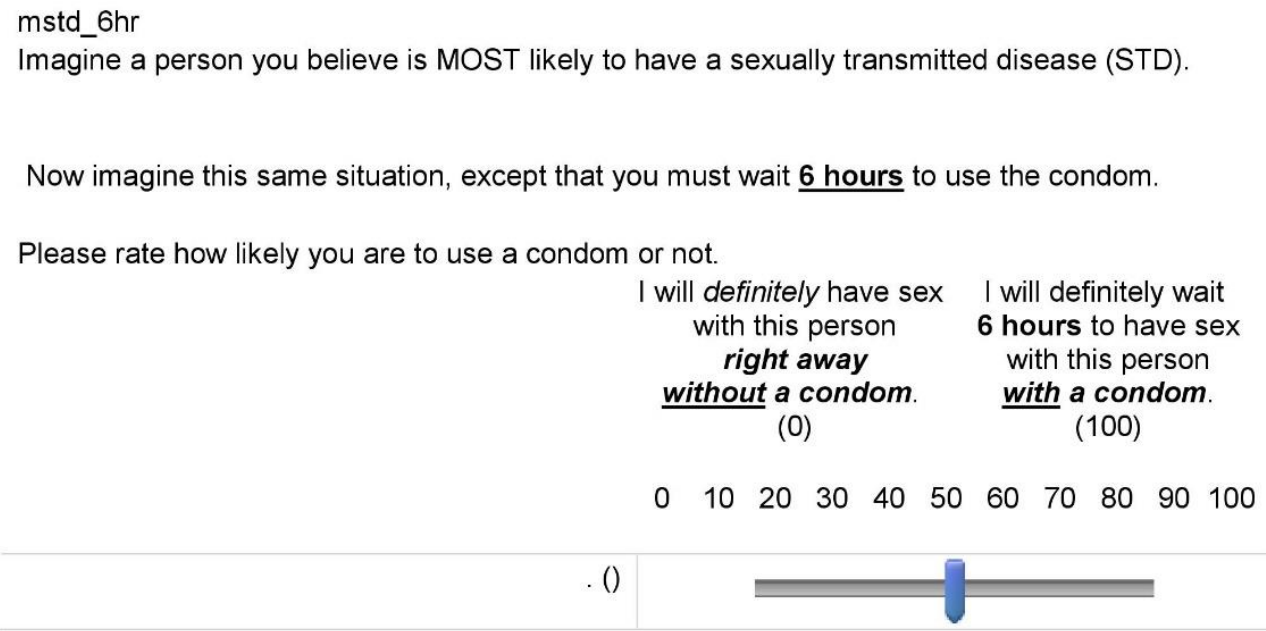

Page Break

Page 64 of 100 
End of Block: Mstd

Start of Block: Lstd

Istd_attract

NOW, Imagine a person you believe is LEAST likely to have a sexually transmitted disease (STD).

How would you rate this individual's attractiveness?

Very UNattractive Very Attractive

(0)

(100)

$\begin{array}{lllllllllll}0 & 10 & 20 & 30 & 40 & 50 & 60 & 70 & 80 & 90 & 100\end{array}$

()

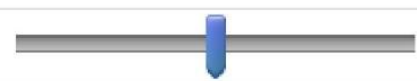

Page Break

Page 65 of 100 
Istd_0

Imagine a person you believe is LEAST likely to have a sexually transmitted disease (STD).

Imagine that you have just met this person. You are getting along great and they are interested in having sex with you now.

Imagine you are confident that there is no chance of pregnancy, for example, you know that one of you is either on the pill, has had their "tubes tied", or had a vasectomy.

Imagine that there is a condom readily and immediately available.

Please rate how likely you are to use a condom or not.

I will definitely have sex I will definitely have sex with this person with this person

right away right away

without a condom. with a condom.

(0)

(100)

$\begin{array}{lllllllllll}0 & 10 & 20 & 30 & 40 & 50 & 60 & 70 & 80 & 90 & 100\end{array}$

()

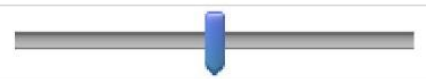

Page Break

Page 66 of 100 
Istd_2min

Imagine a person you believe is LEAST likely to have a sexually transmitted disease (STD).

Now imagine this same situation, except that you must wait $\underline{\mathbf{2} \text { minutes }}$ to use the condom.

Please rate how likely you are to use a condom or not.

I will definitely have sex I will definitely wait with this person 2 minutes to have sex right away with this person without a condom. with a condom. (0)

$\begin{array}{lllllllllll}0 & 10 & 20 & 30 & 40 & 50 & 60 & 70 & 80 & 90 & 100\end{array}$

()

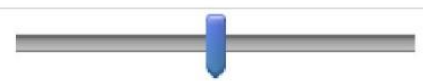

Page Break

Page 67 of 100 


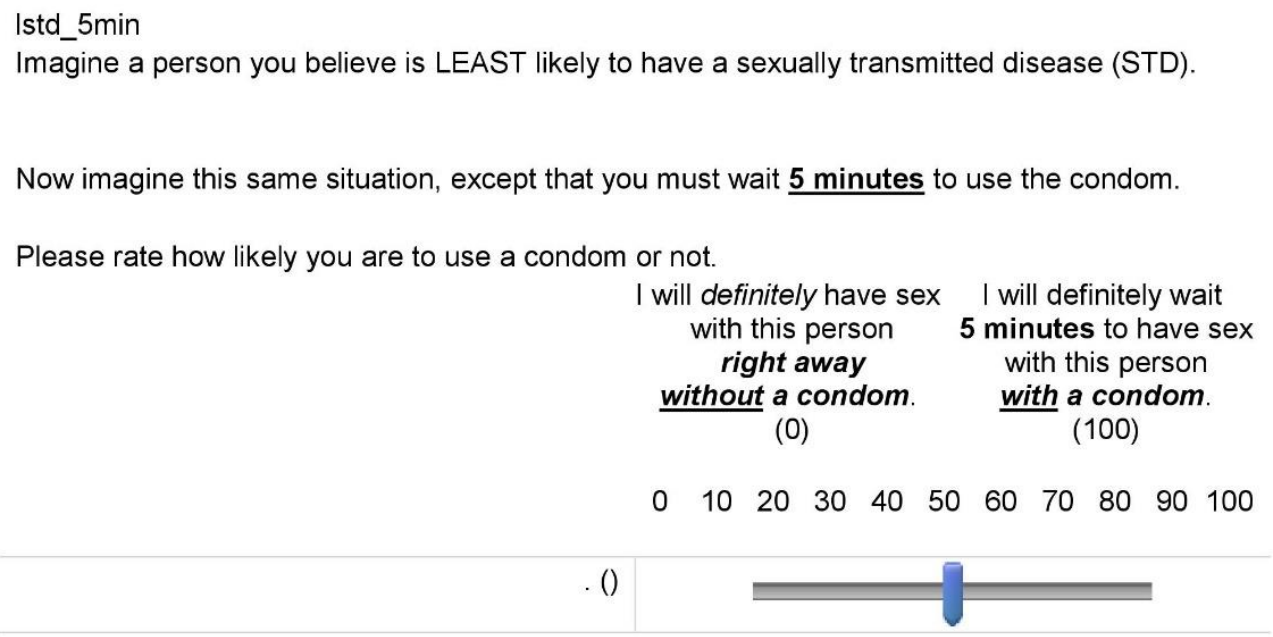

Page Break

Page 68 of 100 
Istd_15min

Imagine a person you believe is LEAST likely to have a sexually transmitted disease (STD).

Now imagine this same situation, except that you must wait $\underline{15 \text { minutes }}$ to use the condom.

Please rate how likely you are to use a condom or not.

I will definitely have sex with this person right away without a condom.

I will definitely wait 15 minutes to have sex with this person with a condom.

$\begin{array}{lllllllllll}0 & 10 & 20 & 30 & 40 & 50 & 60 & 70 & 80 & 90 & 100\end{array}$ ()

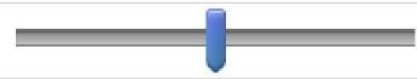




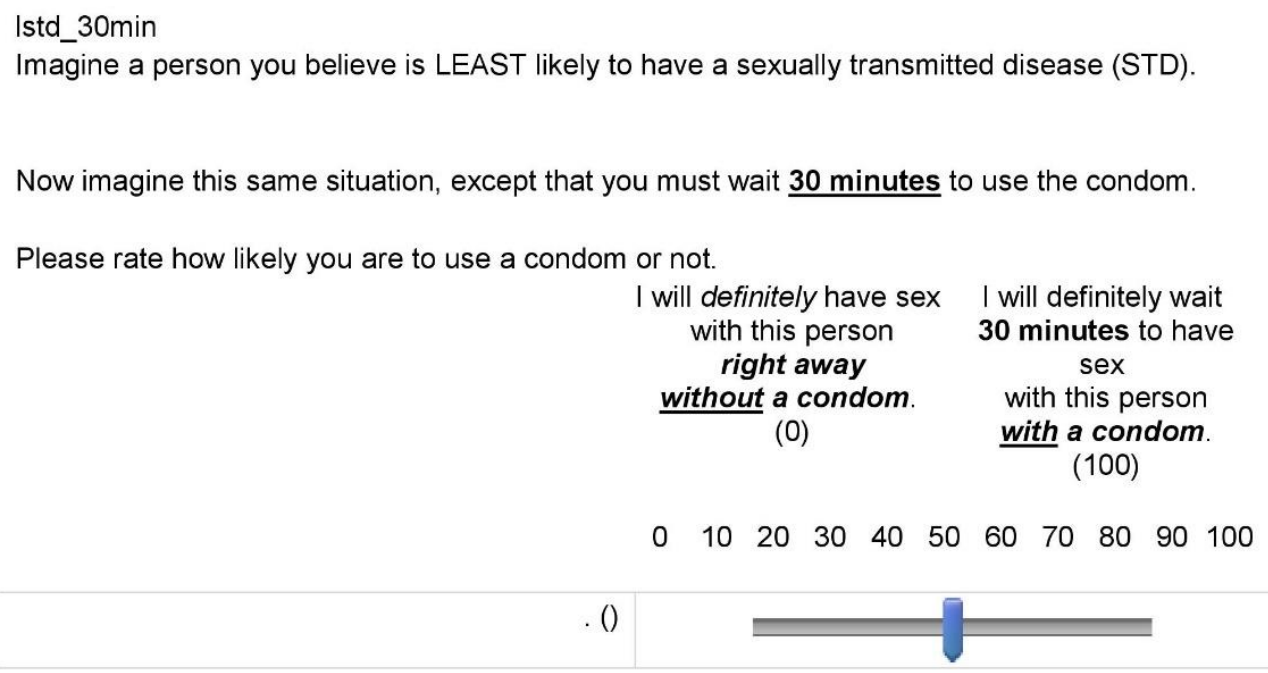




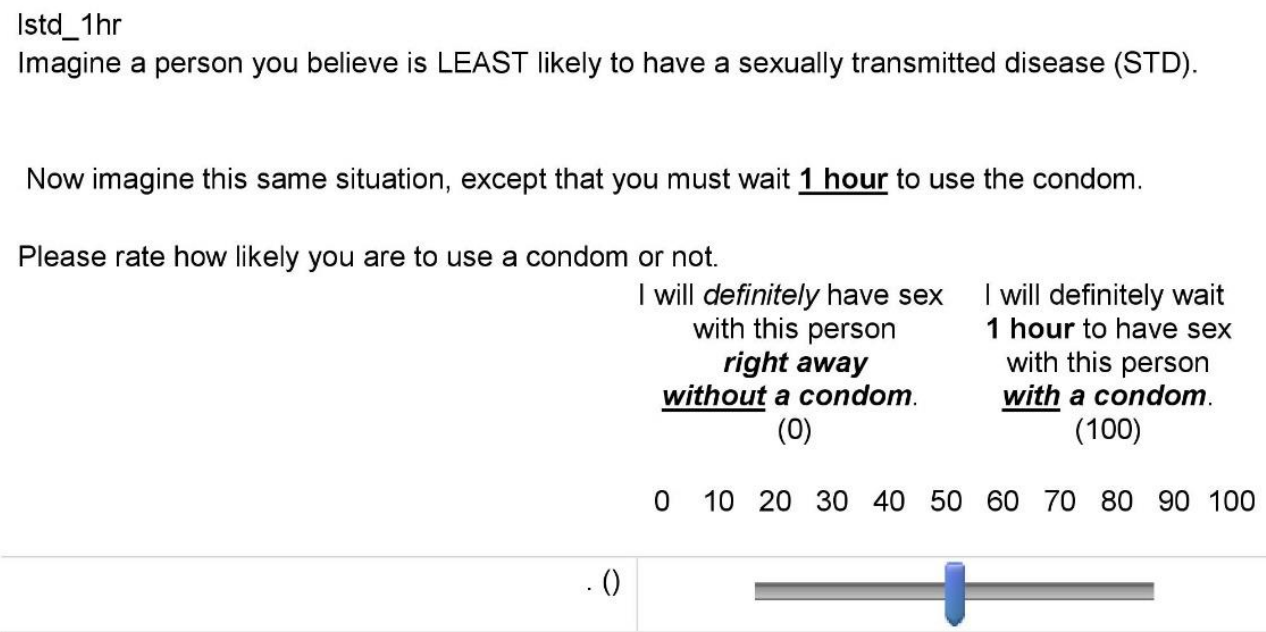

Page Break

Page 71 of 100 


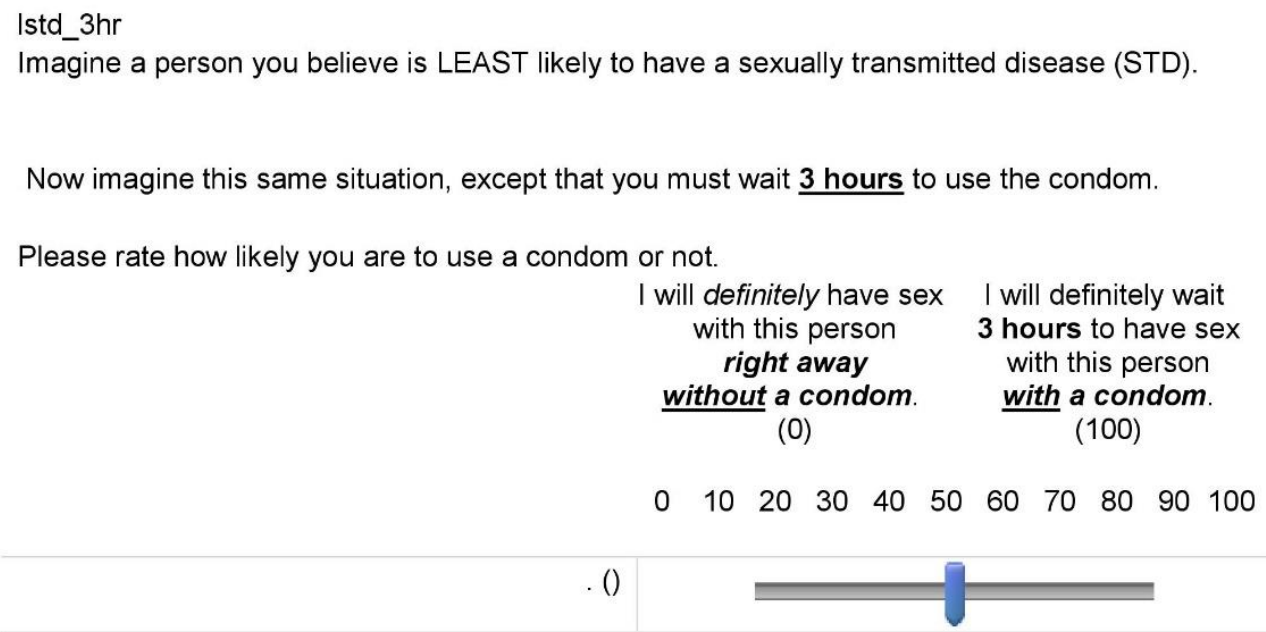

Page Break

Page 72 of 100 
Istd_6hr

Imagine a person you believe is LEAST likely to have a sexually transmitted disease (STD).

Now imagine this same situation, except that you must wait $\underline{6 \text { hours }}$ to use the condom.

Please rate how likely you are to use a condom or not.
I will definitely have sex I will definitely wait with this person $\quad 6$ hours to have sex
right away with this person
without a condom. with a condom.

$\begin{array}{lllllllllll}0 & 10 & 20 & 30 & 40 & 50 & 60 & 70 & 80 & 90 & 100\end{array}$

()

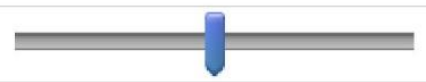

End of Block: Lstd

Start of Block: Pre_preg Reminder Block

prepreg_intro1 For each person you think about, we would like you to think about how attractive that person is based on physical appearance alone. Please think about whether each person is someone that you would consider having sex with in the right environment and if you liked the person's personality.

prepreg_intro2

For the next task, we will ask you hypothetical (pretend) questions about your willingness to have sex in various situations.

For the purpose of this task, please pretend that you are NOT currently in a committed sexual relationship if you are.

In other words, please pretend that you are single and available, and that you are not cheating on anybody if you indicate you would have sex with somebody in this task. 


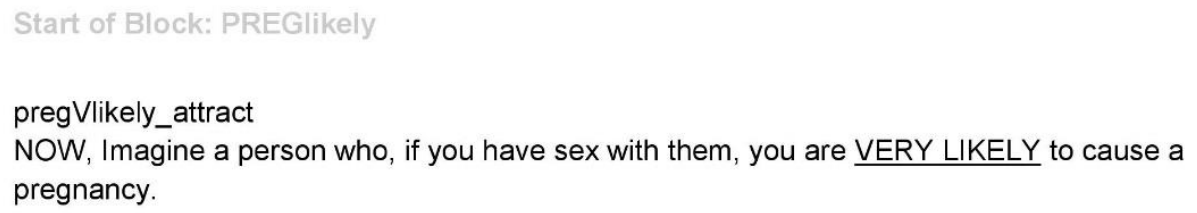


pregVlikely_0

Imagine a person who, if you have sex with them, you are VERY LIKELY to cause a pregnancy.

Imagine that you have just met this person. You are getting along great and they are interested in having sex with you now.

Imagine you are confident that having sex with this person is VERY LIKELY to cause

a pregnancy.

Imagine that there is a condom readily and immediately available.

Please rate how likely you are to use a condom or not.

I will definitely have sex I will definitely have sex with this person with this person

right away right away

without a condom.

(0)

with a condom.

(100)

$\begin{array}{lllllllllll}0 & 10 & 20 & 30 & 40 & 50 & 60 & 70 & 80 & 90 & 100\end{array}$

. ()

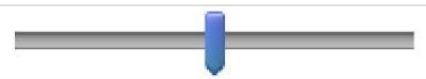

Page Break

Page 75 of 100 


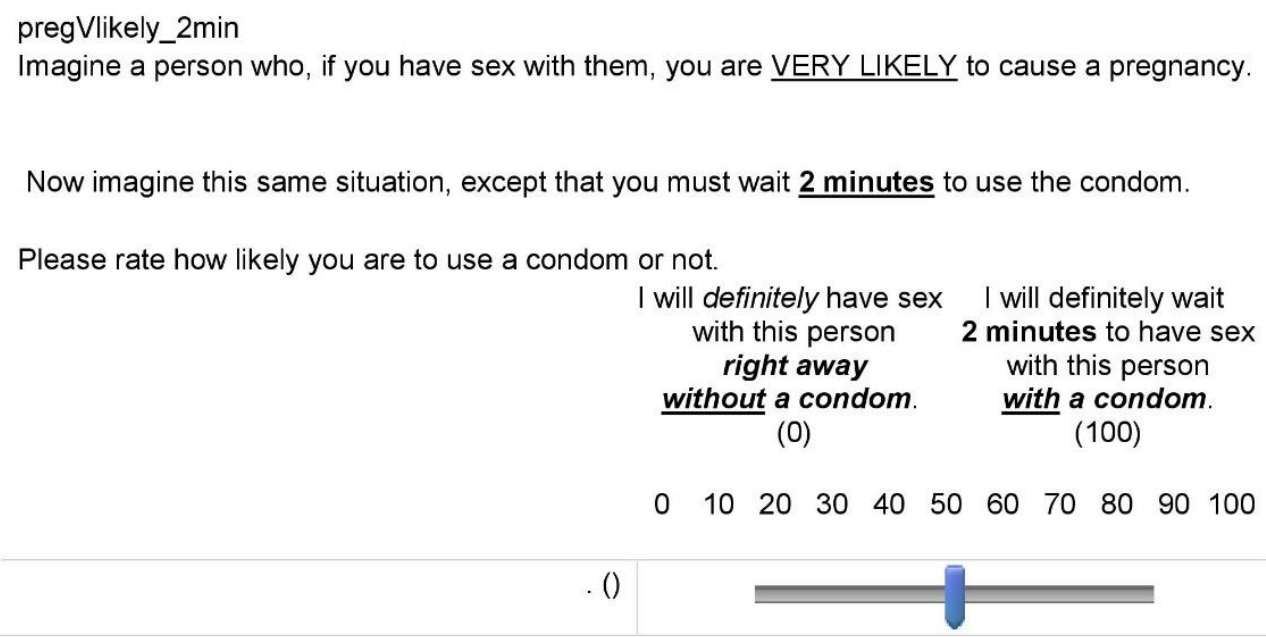

Page Break

Page 76 of 100 


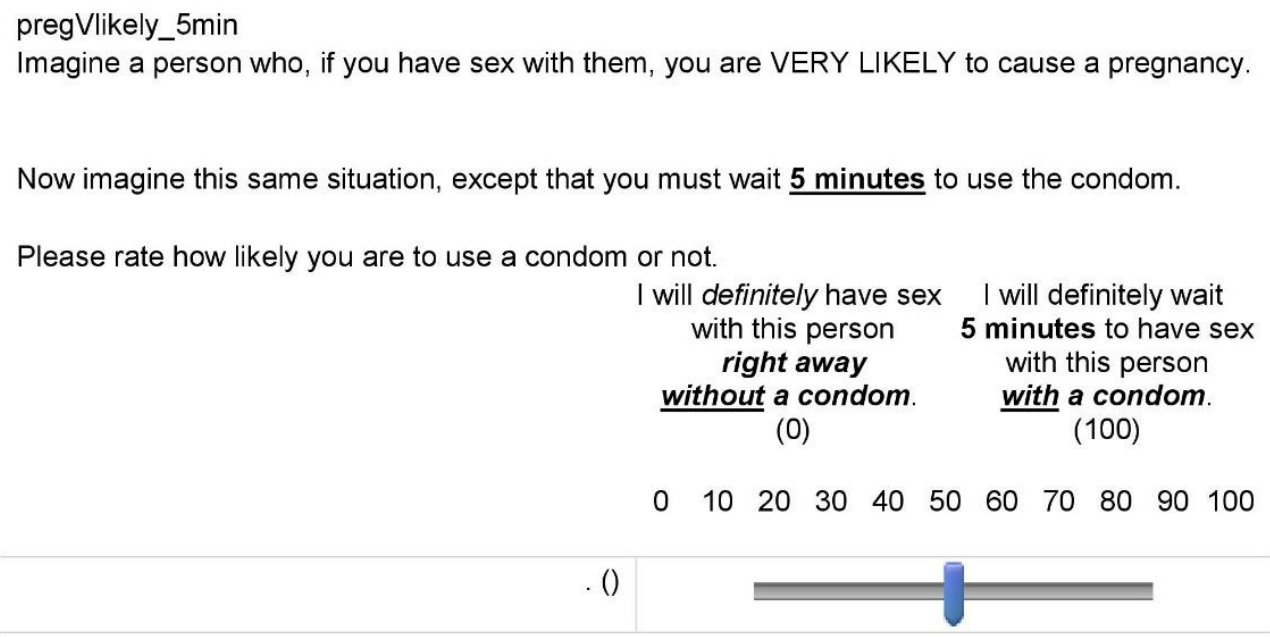

Page Break

Page 77 of 100 
pregVlikely_15min
Imagine a person who, if you have sex with them, you are VERY LIKELY to cause a pregnancy.

Now imagine this same situation, except that you must wait $\underline{15 \text { minutes }}$ to use the condom.

Please rate how likely you are to use a condom or not.

I will definitely have sex with this person right away without a condom.

I will definitely wait 15 minutes to have sex with this person with a condom.

(100)

$\begin{array}{lllllllllll}0 & 10 & 20 & 30 & 40 & 50 & 60 & 70 & 80 & 90 & 100\end{array}$

()

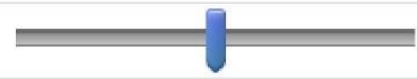


pregVlikely_30min

Imagine a person who, if you have sex with them, you are VERY LIKELY to cause a pregnancy.

Now imagine this same situation, except that you must wait $\underline{\mathbf{3 0} \text { minutes }}$ to use the condom.

Please rate how likely you are to use a condom or not.

I will definitely have sex with this person right away without a condom.

I will definitely wait 30 minutes to have sex with this person with a condom.

$\begin{array}{lllllllllll}0 & 10 & 20 & 30 & 40 & 50 & 60 & 70 & 80 & 90 & 100\end{array}$ ()

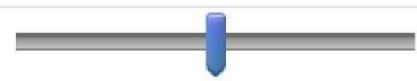




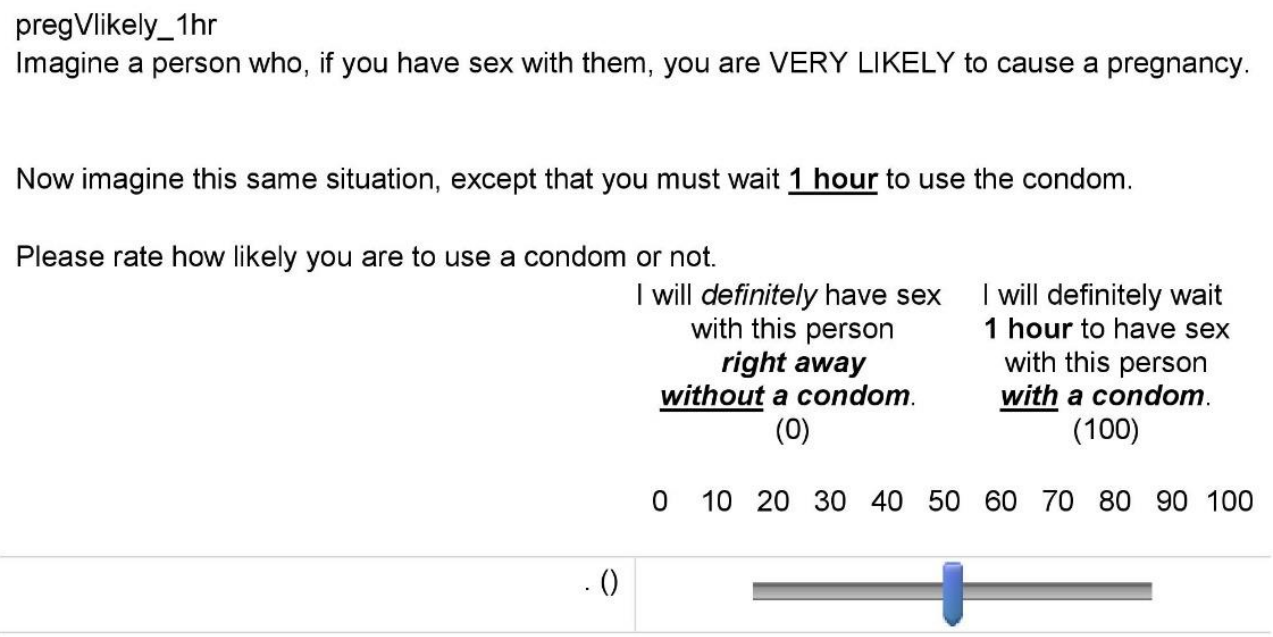

Page Break

Page 80 of 100 


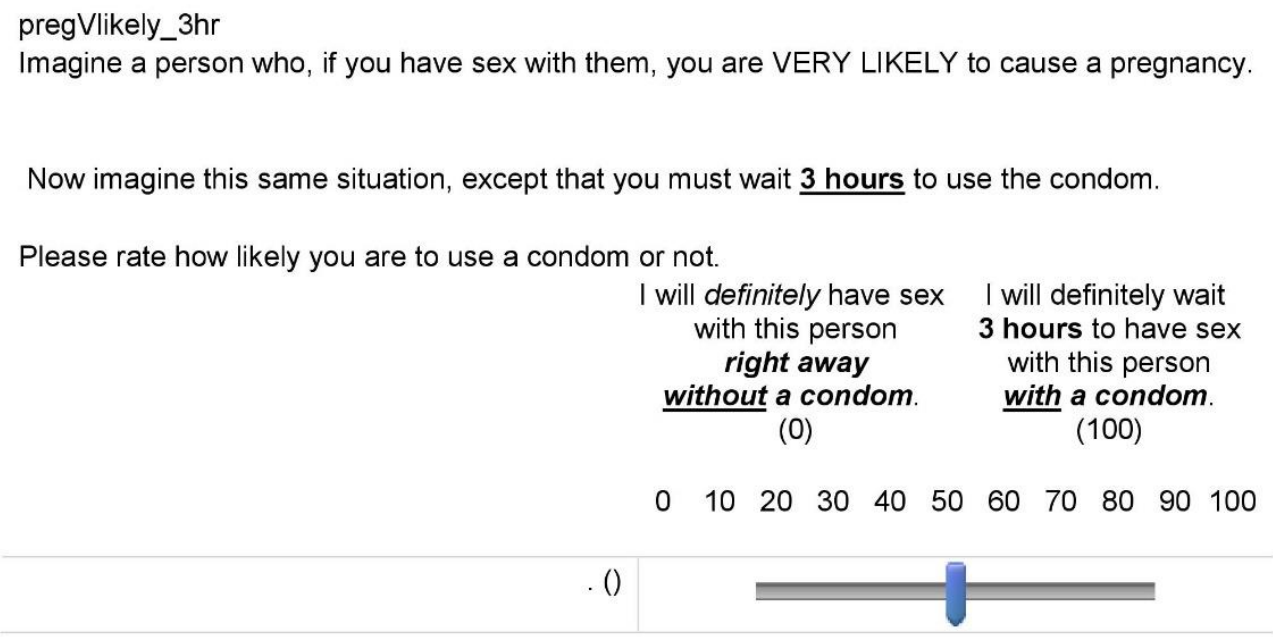

Page Break

Page 81 of 100 


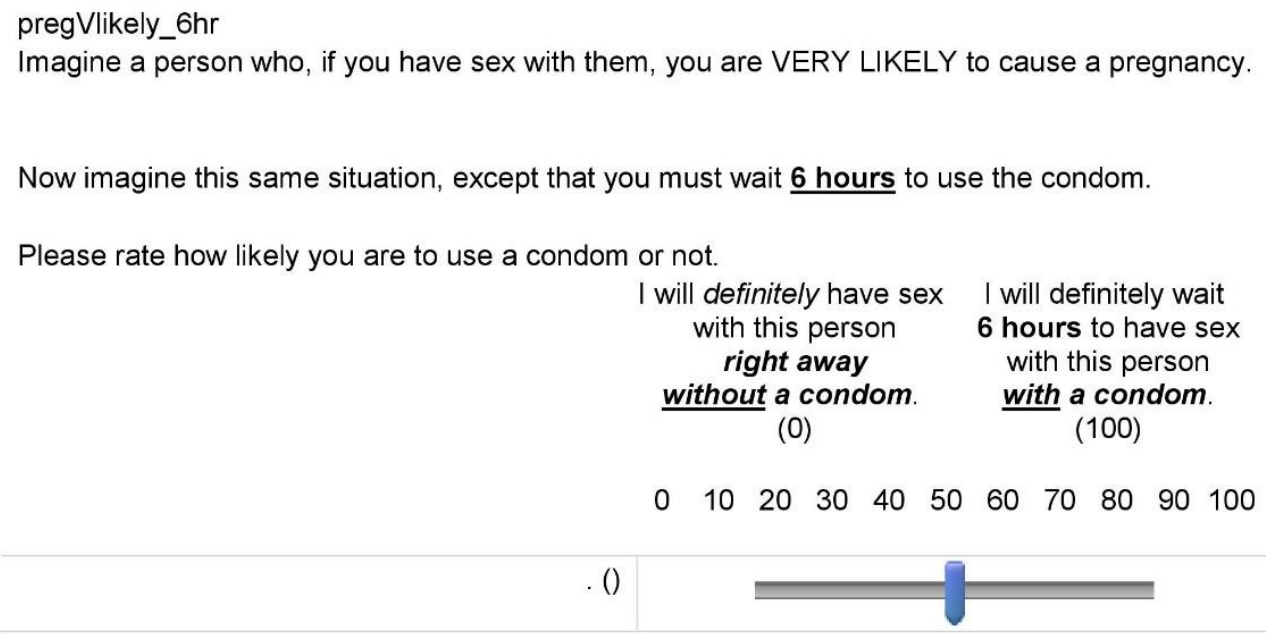

End of Block: PREGlikely

Start of Block: PREGUNlikely

pregUNlikely_attract

NOW, Imagine a person who, if you have sex with them, you are VERY UNlikely to cause a pregnancy.

How would you rate this individual's attractiveness?

$$
\text { Very UNattractive Very Attractive }
$$

$\begin{array}{lllllllllll}0 & 10 & 20 & 30 & 40 & 50 & 60 & 70 & 80 & 90 & 100\end{array}$ 


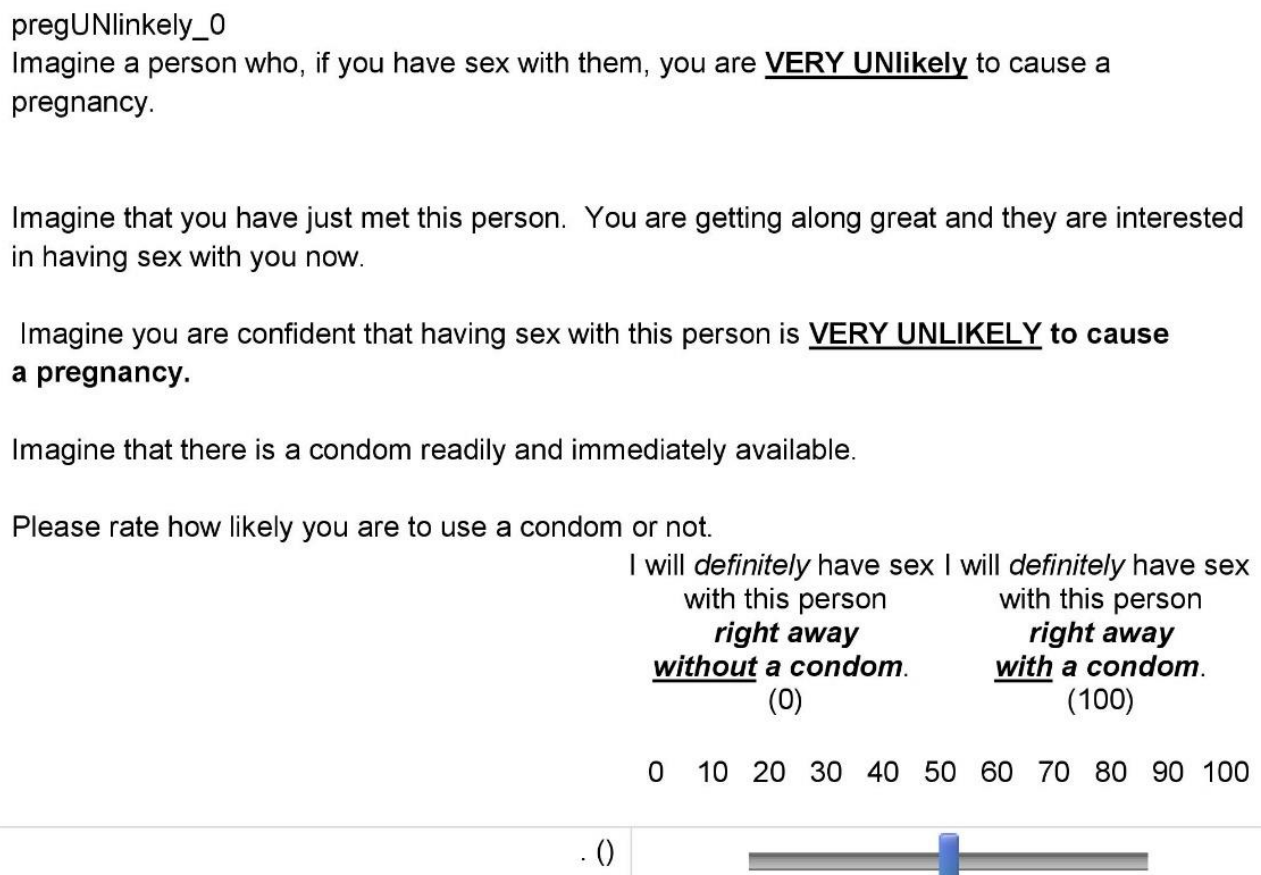




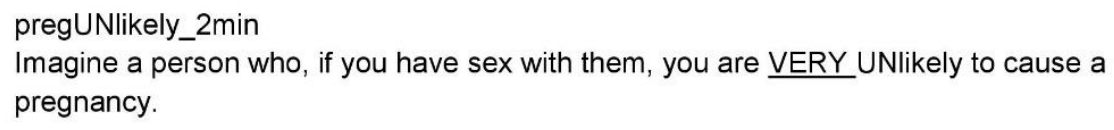

Now imagine this same situation, except that you must wait $\underline{\mathbf{2} \text { minutes }}$ to use the condom.

Please rate how likely you are to use a condom or not.

I will definitely have sex I will definitely wait with this person 2 minutes to have sex right away with this person without a condom. with a condom. (0) (100)

$\begin{array}{lllllllllll}0 & 10 & 20 & 30 & 40 & 50 & 60 & 70 & 80 & 90 & 100\end{array}$

()

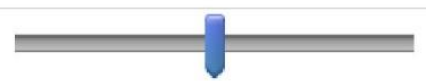

Page Break 


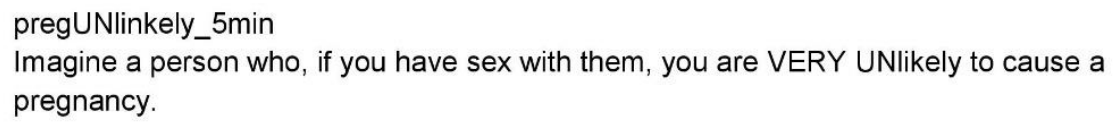

Now imagine this same situation, except that you must wait $\underline{\mathbf{5} \text { minutes }}$ to use the condom.

Please rate how likely you are to use a condom or not.

I will definitely have sex I will definitely wait with this person 5 minutes to have sex right away with this person without a condom. with a condom. (0) (100)

$\begin{array}{lllllllllll}0 & 10 & 20 & 30 & 40 & 50 & 60 & 70 & 80 & 90 & 100\end{array}$ 


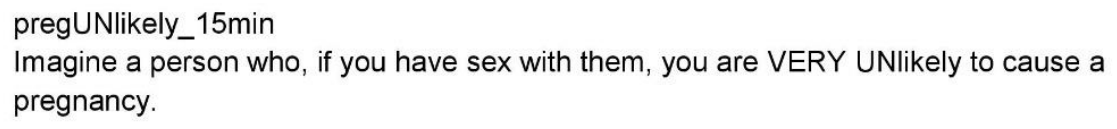

Now imagine this same situation, except that you must wait $\underline{15 \text { minutes }}$ to use the condom.

Please rate how likely you are to use a condom or not.

I will definitely have sex I will definitely wait with this person $\quad 15$ minutes to have right away without a condom (0) sex with this person with a condom. (100)

$\begin{array}{lllllllllll}0 & 10 & 20 & 30 & 40 & 50 & 60 & 70 & 80 & 90 & 100\end{array}$

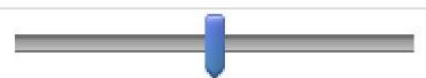




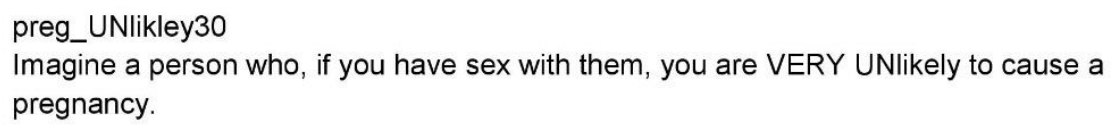

Now imagine this same situation, except that you must wait $\underline{30 \text { minutes }}$ to use the condom.

Please rate how likely you are to use a condom or not.

I will definitely have sex I will definitely wait with this person $\quad \mathbf{3 0}$ minutes to have right away without a condom (0) sex with this person with a condom. (100)

$\begin{array}{lllllllllll}0 & 10 & 20 & 30 & 40 & 50 & 60 & 70 & 80 & 90 & 100\end{array}$ 


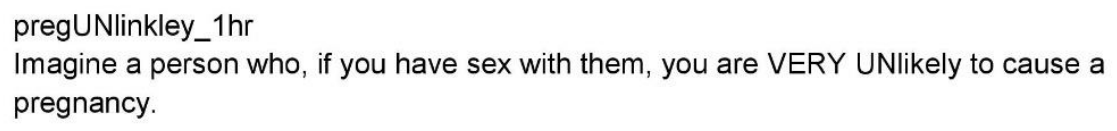

Now imagine this same situation, except that you must wait 1 hour to use the condom.

Please rate how likely you are to use a condom or not.

I will definitely have sex I will definitely wait with this person 1 hour to have sex right away with this person without a condom. with a condom. (0)

(100)

$\begin{array}{lllllllllll}0 & 10 & 20 & 30 & 40 & 50 & 60 & 70 & 80 & 90 & 100\end{array}$

()

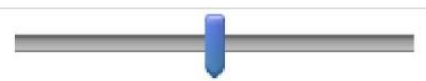

Page Break 


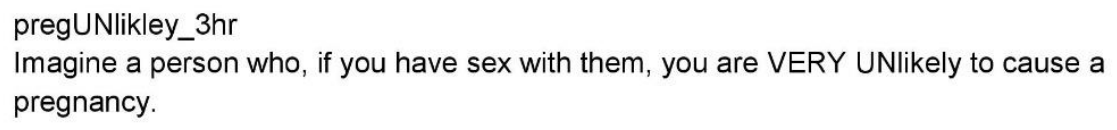

Now imagine this same situation, except that you must wait $\underline{\mathbf{3} \text { hours }}$ to use the condom.

Please rate how likely you are to use a condom or not.

I will definitely have sex I will definitely wait with this person $\quad 3$ hours to have sex right away with this person without a condom. with a condom. (0) (100)

$\begin{array}{lllllllllll}0 & 10 & 20 & 30 & 40 & 50 & 60 & 70 & 80 & 90 & 100\end{array}$

()

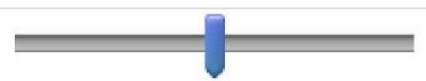

Page Break 


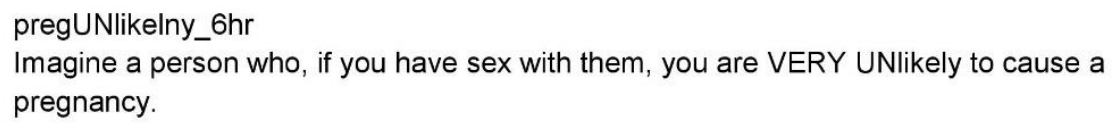

Now imagine this same situation, except that you must wait 6 hours to use the condom.

Please rate how likely you are to use a condom or not.

I will definitely have sex I will definitely wait with this person $\quad 6$ hours to have sex right away with this person without a condom. with a condom.

$\begin{array}{lllllllllll}0 & 10 & 20 & 30 & 40 & 50 & 60 & 70 & 80 & 90 & 100\end{array}$

()

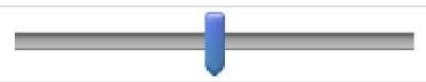

End of Block: PREGUNlikely

Start of Block: Final Questions - Male

rotate 2 Please rotate the tablet to be height-ways. There are no more questions with a slider.

overall_sti_worry Overall, how worried are you about STDs/STIs?

A lot (1)

Somewhat (5)

A little (4)

Not at all (6) 
sexlife_satisfaction Thinking about your sex life in the last year, how much do you agree or disagree with the following statement:

"I feel satisfied with my sex life"'

Strongly agree (13)

Somewhat agree (14)

Neither agree nor disagree (15)

Somewhat disagree (16)

Strongly disagree (17)

condomuse_percent_li Which one of the following percentages best describes the percent of times you had intercourse and you used a condom in your lifetime?

$1 \%-10 \%$ of the time (1)

$11 \%-30 \%$ of the time $(2)$

$31 \%-50 \%$ of the time (3)

$51 \%-70 \%$ of the time $(11)$

$71 \%-90 \%$ of the time $(12)$

$91 \%-99.9 \%$ of the time $(13)$

$100 \%$ of the time - I have always used a condom in my lifetime (14)

$0 \%$ of the time - I have never used a condom in my lifetime (15) 
condomuse_percent_12 Which one of the following percentages best describes the percent of times you had intercourse and you used a condom in the past 12 months?

$1 \%-10 \%$ of the time (1)

$11 \%-30 \%$ of the time (2)

$31 \%-50 \%$ of the time (3)

$51 \%-70 \%$ of the time (11)

$71 \%-90 \%$ of the time $(12)$

$91 \%-99.9 \%$ of the time $(13)$

$100 \%$ of the time - I did always used a condom in the past 12 months (14)

$0 \%$ of the time - I did not use a condom in the past 12 months (15)

ID_STI How easy would it be for you to identify whether a potential sexual partner has an STI, without asking?

Very easy (1)

Easy (2)

Neither easy nor difficult (3)

Difficult (4)

Very difficult (5)

TY1 Thank you for participating in this part of the survey!

End of Block: Final Questions - Male

Start of Block: Final Questions - Female

Page 95 of 100 


\section{Appendix B}

\section{Funding information.}

Initial funding provided by Behavioral Medicine and Psychiatry at West Virginia University was used toward pilot testing Wave 1 of the study to determine interest and feasibility. After initial pilot testing, the protocol was revised and resubmitted to the IRB. Participants who were recruited and consented during the pilot testing phase were re-consented at Wave 2. All other participants were consented at Wave 1. Data for this project is primarily from Wave 2. Before Wave 2 was administered, participants were reminded about the task procedures and questions were answered.

\section{Pilot testing information.}

Data from the first 50 women participants were used to pilot test the survey and technology to determine: ease of use, overall survey length, and acceptability. Questions were clarified using pilot participants' input. Colloquial terms (e.g., rubbers, condoms) were used when possible to enhance readability and clarify the medical terminology included in the survey. No significant issues were identified with the final three survey waves, each of which took about 15 minutes to complete. The Flesch-Kincaid index suggested a six-grade reading level (index = 6.3). Adults who were willing and eligible could complete Wave 1, Wave 1 and Wave 2 on the same day, Wave 2 separately, or Wave 2 and Wave 3 on the same day. Time always separated Wave 1 and Wave 3 to allow measurement of behavior change between Wave 1 and Wave 3 . Not all participants completed all study Waves.

\section{Data collection tools.}

REDCap (Research Electronic Data Capture) is a secure, web-based application designed to support data capture for research studies, providing 1) an intuitive interface for validated data 
entry; 2) audit trails for tracking data manipulation and export procedures; 3) automated export procedures for seamless data downloads to common statistical packages; and 4) procedures for importing data from external sources (Harris et al., 2009). The shared data instrument tool was used in REDCAP for data collection (Obeid et al., 2013).

\section{Dinner Task: Practice.}

After demographics questions in Wave 2, a practice session was used to acclimate participants to the question format, identify any questions participants had about the task, identify problems with the using the VAS on the tablet, and emphasize reading the prompt for each portion of the task. Previous studies have also used a simulated practice session to allow for questions and determine ability to complete the task (Johnson et al., 2017).

The practice questions asked participants to rate "how likely are you to eat dinner tonight?" and instructed participants to move the VAS pointer to a specific percent. The fixed percent responses included: $0 \%, 5 \%, 25 \%, 50 \%, 75 \%, 95 \%$, and $100 \%$. Both poles of the VAS were labeled as either "I am definitely not eating dinner tonight ( $0 \%)$ " and "I am definitely eating dinner tonight (100\%)." Participants were not able to advance to the next delay or task until they correctly moved the VAS pointer to the pre-determined location described in the prompt. The practice task was not included in any analysis as the responses were fixed and all participants completed this task prior to moving on to the other delay discounting tasks. 


\section{Appendix C}

Randomization procedures:

Monetary Delay-Discounting Task - presented $1^{\text {st. }}$

\begin{tabular}{|l|l|}
\hline Block 1 $(n=51)$ & Block 2 $(n=62)$ \\
\hline \$10 Monetary & \$100 Monetary \\
Task & Task \\
\hline \$100 Monetary & \$10 Monetary \\
Task & Task \\
\hline
\end{tabular}

Sexual Discounting Task Attractiveness and STD Risk - blocks were randomly presented from the options below:

\begin{tabular}{|c|c|c|c|c|c|c|c|}
\hline $\begin{array}{l}\text { Block 1 } \\
(n=10)\end{array}$ & $\begin{array}{l}\text { Block } 2 \\
(n=11)\end{array}$ & $\begin{array}{l}\text { Block } 3 \\
(n=12)\end{array}$ & $\begin{array}{l}\text { Block } 4 \\
(n=14)\end{array}$ & $\begin{array}{l}\text { Block 5 } \\
(n=11)\end{array}$ & $\begin{array}{l}\text { Block } 6 \\
(n=13)\end{array}$ & $\begin{array}{l}\text { Block } 7 \\
(n=10)\end{array}$ & $\begin{array}{l}\text { Block } 8 \\
(n=15)\end{array}$ \\
\hline $\begin{array}{l}\text { Attractiv } \\
\text { eness } \\
\text { (High) }\end{array}$ & $\begin{array}{l}\text { Attractiv } \\
\text { eness } \\
\text { (High) }\end{array}$ & $\begin{array}{l}\text { Attractiv } \\
\text { eness } \\
\text { (Low) }\end{array}$ & $\begin{array}{l}\text { Attractiv } \\
\text { eness } \\
\text { (Low) }\end{array}$ & $\begin{array}{l}\text { STD } \\
\text { Risk } \\
\text { (High) }\end{array}$ & $\begin{array}{l}\text { STD } \\
\text { Risk } \\
\text { (High) }\end{array}$ & $\begin{array}{l}\text { STD } \\
\text { Risk } \\
\text { (Low) } \\
\end{array}$ & $\begin{array}{l}\text { STD } \\
\text { Risk } \\
\text { (Low) } \\
\end{array}$ \\
\hline $\begin{array}{l}\text { Attractiv } \\
\text { eness } \\
\text { (Low) }\end{array}$ & $\begin{array}{l}\text { Attractiv } \\
\text { eness } \\
\text { (Low) }\end{array}$ & $\begin{array}{l}\text { Attractiv } \\
\text { eness } \\
\text { (High) }\end{array}$ & $\begin{array}{l}\text { Attractiv } \\
\text { eness } \\
\text { (High) }\end{array}$ & $\begin{array}{l}\text { STD } \\
\text { Risk } \\
\text { (Low) }\end{array}$ & $\begin{array}{l}\text { STD } \\
\text { Risk } \\
\text { (Low) }\end{array}$ & $\begin{array}{l}\text { STD } \\
\text { Risk } \\
\text { (High) }\end{array}$ & $\begin{array}{l}\text { STD } \\
\text { Risk } \\
\text { (High) }\end{array}$ \\
\hline $\begin{array}{l}\text { Validity } \\
\text { Check }\end{array}$ & $\begin{array}{l}\text { Validity } \\
\text { Check }\end{array}$ & $\begin{array}{l}\text { Validity } \\
\text { Check }\end{array}$ & $\begin{array}{l}\text { Validity } \\
\text { Check }\end{array}$ & $\begin{array}{l}\text { Validity } \\
\text { Check }\end{array}$ & $\begin{array}{l}\text { Validity } \\
\text { Check }\end{array}$ & $\begin{array}{l}\text { Validity } \\
\text { Check }\end{array}$ & $\begin{array}{l}\text { Validity } \\
\text { Check }\end{array}$ \\
\hline $\begin{array}{l}\text { STD } \\
\text { Risk } \\
\text { (High) }\end{array}$ & $\begin{array}{l}\text { STD } \\
\text { Risk } \\
\text { (Low) }\end{array}$ & $\begin{array}{l}\text { STD } \\
\text { Risk } \\
\text { (High) }\end{array}$ & $\begin{array}{l}\text { STD } \\
\text { Risk } \\
\text { (Low) }\end{array}$ & $\begin{array}{l}\text { Attractiv } \\
\text { eness } \\
\text { (High) }\end{array}$ & $\begin{array}{l}\text { Attractiv } \\
\text { eness } \\
\text { (Low) }\end{array}$ & $\begin{array}{l}\text { Attractiv } \\
\text { eness } \\
\text { (Low) }\end{array}$ & $\begin{array}{l}\text { Attractiv } \\
\text { eness } \\
\text { (High) }\end{array}$ \\
\hline $\begin{array}{l}\text { STD } \\
\text { Risk } \\
\text { (Low) }\end{array}$ & $\begin{array}{l}\text { STD } \\
\text { Risk } \\
\text { (High) }\end{array}$ & $\begin{array}{l}\text { STD } \\
\text { Risk } \\
\text { (Low) }\end{array}$ & $\begin{array}{l}\text { STD } \\
\text { Risk } \\
\text { (High) }\end{array}$ & $\begin{array}{l}\text { Attractiv } \\
\text { eness } \\
\text { (Low) }\end{array}$ & $\begin{array}{l}\text { Attractiv } \\
\text { eness } \\
\text { (High) }\end{array}$ & $\begin{array}{l}\text { Attractiv } \\
\text { eness } \\
\text { (High) }\end{array}$ & $\begin{array}{l}\text { Attractiv } \\
\text { eness } \\
\text { (Low) }\end{array}$ \\
\hline
\end{tabular}

Sexual Discounting Task Pregnancy Risk - blocks were randomly presented from the options below:

\begin{tabular}{|l|l|}
\hline Block 1 $(n=62)$ & Block 2 $(n=51)$ \\
\hline Pregnancy Risk (Low) & Pregnancy Risk (High) \\
\hline Validity Check & Validity Check \\
\hline Pregnancy Risk (High) & Pregnancy Risk (Low) \\
\hline
\end{tabular}




\section{Appendix D}

\section{Monetary Delay Discounting Task AUC formula.}
$\mathrm{AUC}=\left(5^{*}(5 \mathrm{~min}\right.$ delay $+10 \mathrm{~min}$ delay $\left.) / 2\right)+\left(20^{*}(10 \mathrm{~min}\right.$ delay $+30 \mathrm{~min}$ delay $\left.) / 2\right)+$ $(30 *(30$ min delay +60 min delay $) / 2)+(120 *(60$ min delay +180 min delay $) / 2)+$ $(180 *(180$ min delay +360 min delay $) / 2)+(360 *(360$ min delay +720 min delay $) / 2)+$ $(720 *(720$ min delay +1440 min delay $) / 2)$

\section{Sexual Discounting Task AUC formula.}

$\mathrm{AUC}=(3 *([2 \mathrm{~min}$ delay $/ 0 \mathrm{~min}$ delay $]+[5 \mathrm{~min}$ delay $/ 0 \mathrm{~min}$ delay $]) / 2)+(10 *([5 \mathrm{~min}$ delay/0 min delay $]+[15 \mathrm{~min}$ delay $/ 0 \mathrm{~min}$ delay $]) / 2)+\left(15^{*}([15 \mathrm{~min}\right.$ delay/0 $\min$ delay $]+$ $[30 \mathrm{~min}$ delay/0 min delay $]) / 2)+(30 *([30 \mathrm{~min}$ delay/0 min delay $]+[60 \mathrm{~min}$ delay/0 $\mathrm{min}$ delay $]) / 2)+(120 *([60 \mathrm{~min}$ delay/0 min delay $]+[180 \mathrm{~min}$ delay/0 min delay $]) / 2)+$ $(180 *([180 \mathrm{~min}$ delay/0 min delay $]+[360 \mathrm{~min}$ delay/0 min delay $]) / 2)$ 


\section{Appendix E}

\section{Omnibus Testing}

Three Friedman's non-parametric omnibus tests was computed to determine overall median differences in this repeated measure design. First, a comparison was computed between Raw AUC for all the discounting conditions (i.e., High/Low Attraction, High/Low STD Risk, High/Low Pregnancy Risk). There was a statistically significant difference in Raw AUC across conditions, $\chi^{2}(5)=34.3, p<.001$. Second, a comparison was computed between "Immediately Available" Condom Preference for all the discounting conditions (i.e., 0-delay for each Sexual Discounting Task condition High/Low Attraction, High/Low STD Risk, High/Low Pregnancy Risk). There was a statistically significant difference in "Immediately Available" condom preference across Sexual Discounting Task conditions, $\chi^{2}(5)=14.2, p=.014$. Third, a comparison was computed between Standardized AUC for all the discounting conditions. There was a statistically significant difference in Standardized AUC across Sexual Discounting Task conditions, $\chi^{2}(5)=32.9, p<.001$. Wilcoxon Signed-ranks Tests was computed to probe differences between domains and within conditions.

\section{Standardized Sexual Delay Discounting Analysis.}

To account for individual condom preferences and separate the effect of delay on condom preference, the Sexual Discounting Task AUC values were standardized using the response at 0delay (Herrmann et al., 2014; Johnson et al., 2017). To compute the standardized AUC, raw values are converted to standardized responses (i.e., raw value at time point X/0-delay) for each time point. These standardized values are then used to compute a series of trapezoids to form the final AUC value (Figure 2). For each participant, condition, and temporal delay, the standardized condom preference was used to calculate AUC. Standardized AUC values over 1.0 were rounded 
down to 1.0 (M. W. Johnson et al., 2015). This can happen when subsequent delays are higher than the previous delay, but do not meet Criterion 1 rules (e.g.; 50\%, 51\%, 51\%). A participant could have a Standardized AUC greater than 1 with if their initial preference was lower than other timepoints, but not such that there was a violation of Criterion 1 (i.e., a $\geq 30 \%$ increase between sequential timepoints). For example, a participant with an "Immediately Available" condom use preference of $50 \%$ and subsequent condom use preferences of $55 \%$ would not meet a violation of Criterion 1 but would have higher than 1 for Standardized AUC and thus, were rounded to 1 .

When computing AUC for the Sexual Discounting Task, the "immediately available" delay is used to standardize each participant score based on their overall likelihood to use a condom. For example, if a participant reports that in the MOST attractive condition they are $70 \%$ likely to use a condom if "immediately available," each of the responses for the other delays will be divided by $70 \%$. This approach is used to isolate effects of delay on decisionmaking and remove the influence of individual condom preference. Due to this approach, any participant reporting $0 \%$ likelihood of using an "immediately available" condom will be excluded from analysis using the standardized AUC (Herrmann et al., 2014). If AUC values are over 1 and there is not a Criterion 1 violation, the AUC will be rounded down to 1.

To examine Research Question 1. Are there domain differences in delay discounting? a Wilcoxon Signed-rank Test will be used. To test Research Question 1c. comparisons will be run between Standardized AUC Monetary Delay-Discounting Task (\$10 and \$100) and each Sexual Discounting Task condition Raw AUC (i.e., attractiveness, STD risk, pregnancy risk).

Standardized AUC. Standardized AUC isolate the effect of delay on hypothetical condom use preference. Participants with an "immediately available" preference of $0 \%$ are not 
able to have their Standardized AUC computed because standardization consists of dividing each timepoint by the "immediately available" timepoint. An "immediately available" preference of $0 \%$ indicates that at zero delay, they would hypothetically not use a condom and therefore, are not sensitive to the delays, which is the purpose of computing the Standardized AUC. Because of this, the Standardized AUC the sample size vary: Attraction (High), $n=105$; Attraction (Low), $n$ $=115 ;$ STD $($ High $), n=117 ;$ STD $($ Low $), n=111 ;$ Pregnancy $($ High $), n=120 ;$ Pregnancy (Low), $n=103$.

The Monetary Delay-Discounting Task does not have standardized values because a zero delay is not used for those tasks. As shown in Table 2, the Standardized Attraction (Low), STD (High), STD (Low), Pregnancy (High), and Pregnancy (Low) AUCs were severely negatively skewed. Complete, systematic Raw and Standardized discounting data (e.g., Monetary and Sexual Discounting AUC) were available for 27 participants (21.1\% of the sample). Appendix I includes full results for this sample with complete data.

Research Question 1. Are there domain differences in delay discounting? was broken into three components for analysis, Raw AUC, Standardized AUC, and "Immediately Available" Condom Preferences.

To test Research Question 1a., a series of Wilcoxon Signed-rank Tests was computed between Raw AUC Monetary Delay-Discounting Tasks and each Raw AUC Sexual Discounting Task condition. See Table $3 b$ for a full list of comparisons.

$\$ 10$ Monetary Delay-Discounting Task. The median AUC for the \$10 Monetary DelayDiscounting Task AUC $(\mathrm{Mdn}=.36, \mathrm{IQR}=.82)$ differed from the median Low Attraction Raw AUC $(\mathrm{Mdn}=1.00, \mathrm{IQR}=.19 ; \mathrm{Z}=-2.65, p=.008)$. The $\$ 10$ condition was more steeply discounted than condoms in the Low Attraction condition suggesting a greater preference for 
smaller sooner rewards in the \$10 Monetary condition compared to a greater likelihood of waiting until a condom was available in the Low Attraction condition. See Table $3 \mathrm{~b}$ for a full list of comparisons.

The median AUC for the \$10 Monetary Delay-Discounting Task AUC (Mdn $=.36$, IQR $=.82)$ differed from the median High STD Risk Raw AUC $(\mathrm{Mdn}=1.00, \mathrm{IQR}=.00 ; \mathrm{Z}=-3.81, p$ $<.001)$. The $\$ 10$ condition was more steeply discounted than condoms in the High STD Risk condition suggesting a greater preference for smaller sooner rewards in the $\$ 10$ Monetary condition compared to condoms in the High STD Risk condition. See Table $3 b$ for a full list of comparisons.

The median AUC for the $\$ 10$ Monetary Delay-Discounting Task AUC (Mdn $=.36$, IQR $=.82)$ differed from the median High Pregnancy Risk Raw AUC $(\mathrm{Mdn}=1.00, \mathrm{IQR}=.12 ; \mathrm{Z}=-$ $3.07, p=.002)$. The $\$ 10$ condition was more steeply discounted than condoms in the High Pregnancy Risk condition suggesting a greater preference for smaller sooner rewards in the \$10 Monetary condition compared to condoms in the High Pregnancy Risk condition. See Table 3b for a full list of comparisons.

The median AUC for the $\$ 10$ Monetary Delay-Discounting Task AUC (Mdn $=.36$, IQR $=.82)$ differed from the median Low Pregnancy Risk Raw AUC $(\mathrm{Mdn}=1.00, \mathrm{IQR}=.22 ; \mathrm{Z}=-$ $3.00, p=.003)$. The $\$ 10$ condition was more steeply discounted than condoms in the Low Pregnancy Risk condition suggesting a greater preference for smaller sooner rewards in the $\$ 10$ Monetary condition compared to condoms in the Low Pregnancy Risk condition. See Table 3b for a full list of comparisons.

No significant differences in median AUC were detected between the $\$ 10$ Monetary Delay-Discounting Task and the High Attraction condition $(p=.775)$. Additionally, no 
significant differences in median AUC were detected between the \$10 Monetary DelayDiscounting Task and the Low STD Risk condition $(p=.081)$. See Table $3 b$ for a full list of comparisons.

$\$ 100$ Monetary Delay-Discounting Task. The median AUC for the \$100 Monetary Delay-Discounting Task AUC $(\mathrm{Mdn}=.70, \mathrm{IQR}=.69)$ differed from the median Low Attraction Raw AUC $(\operatorname{Mdn}=1.00, \mathrm{IQR}=.19 ; \mathrm{Z}=-3.58, p<.001)$. The $\$ 100$ condition was less steeply discounted than condoms in the Low Attraction condition suggesting less preference for smaller sooner rewards in the $\$ 100$ Monetary condition compared to condoms in the Low Attraction condition. See Table $3 \mathrm{~b}$ for a full list of comparisons.

The median AUC for the $\$ 100$ Monetary Delay-Discounting Task AUC (Mdn $=.70$, IQR $=.69)$ differed from the median High STD Risk Raw AUC $(\mathrm{Mdn}=1.00, \mathrm{IQR}=.00 ; \mathrm{Z}=-3.82, p$ $<.001)$. The $\$ 100$ was more steeply discounted than condoms in the High STD Risk condition suggesting a greater preference for smaller sooner rewards in the $\$ 100$ Monetary condition compared to condoms in the High STD Risk condition. See Table $3 b$ for a full list of comparisons.

The median AUC for the $\$ 100$ Monetary Delay-Discounting Task AUC (Mdn $=.70$, IQR $=.69)$ differed from the median Low STD Risk Raw AUC $(\mathrm{Mdn}=.92, \mathrm{IQR}=.40 ; \mathrm{Z}=-2.91, p=$ .004). The $\$ 100$ was more steeply discounted than condoms in the Low STD Risk condition suggesting a greater preference for smaller sooner rewards in the $\$ 100$ Monetary condition compared to condoms in the Low STD Risk condition. See Table $3 b$ for a full list of comparisons.

The median AUC for the $\$ 100$ Monetary Delay-Discounting Task AUC (Mdn $=.70$, IQR $=.69)$ differed from the median High Pregnancy Risk Raw AUC (Mdn = 1.00, IQR = .12; Z = - 
$3.82, p<.001)$. The $\$ 100$ condition was more steeply discounted than condoms in the High Pregnancy Risk condition suggesting a greater preference for smaller sooner rewards in the $\$ 100$ Monetary condition compared to condoms in the High Pregnancy Risk condition. See Table $3 b$ for a full list of comparisons.

The median AUC for the $\$ 100$ Monetary Delay-Discounting Task AUC (Mdn $=.70$, IQR $=.69)$ differed from the median Low Pregnancy Risk Raw AUC $(\mathrm{Mdn}=1.00, \mathrm{IQR}=.22 ; \mathrm{Z}=$ $3.91, p<.001)$. The $\$ 100$ condition was more steeply discounted than condoms in the Low Pregnancy Risk condition suggesting a greater preference for smaller sooner rewards in the $\$ 100$ Monetary condition compared to condoms in the Low Pregnancy Risk condition. See Table 3b for a full list of comparisons.

No significant differences in median AUC were detected between the $\$ 100$ Monetary Delay-Discounting Task and the High Attraction condition $(p=.072)$. See Table $3 b$ for a full list of comparisons.

High Attraction Raw AUC. The median AUC for the High Attraction Raw AUC (Mdn = $.78, \mathrm{IQR}=.69)$ differed from the median High STD Risk Raw AUC $(\mathrm{Mdn}=1.00, \mathrm{IQR}=.00 ; \mathrm{Z}$ $=-3.73, p<.001)$. Participants more steeply discounted condoms in the High Attraction compared to the High STD Risk condition. This suggests less preference for using a condom as the delay increased in the High Attraction condition compared to the High STD Risk condition. See Table $3 b$ for a full list of comparisons.

The median AUC for the High Attraction Raw AUC (Mdn = .78, IQR = .69) differed from the median High Pregnancy Risk Raw AUC $(\mathrm{Mdn}=1.00, \mathrm{IQR}=.12 ; \mathrm{Z}=-3.25, p=.001)$. Participants more steeply discounted condoms in the High Attraction compared to the High Pregnancy Risk condition. This suggests less preference for using a condom as the delay 
increased in the High Attraction condition compared to the High Pregnancy Risk condition. See Table $3 b$ for a full list of comparisons.

The median AUC for the High Attraction Raw AUC (Mdn = .78, IQR = .69) differed from the median Low Pregnancy Risk Raw AUC $(\mathrm{Mdn}=1.00, \mathrm{IQR}=.22 ; \mathrm{Z}=-3.49, p<.001)$. Participants more steeply discounted condoms in the High Attraction compared to the Low Pregnancy Risk condition. This suggests less preference for using a condom as the delay increased in the High Attraction condition compared to the Low Pregnancy Risk condition. See Table $3 b$ for a full list of comparisons.

No significant differences in median Raw AUC were detected between the High Attraction condition and the Low STD Risk condition $(p=.117)$. See Table $3 b$ for a full list of comparisons.

Low Attraction Raw AUC. The median AUC for the Low Attraction Raw AUC (Mdn = $1.00, \mathrm{IQR}=.19)$ differed from the median High STD Risk Raw AUC $(\mathrm{Mdn}=1.00, \mathrm{IQR}=.00 ; \mathrm{Z}$ $=-2.28, p=.023)$. Participants more steeply discounted condoms in the Low Attraction compared to the High STD Risk condition. This suggests less preference for using a condom as the delay increased in the Low Attraction condition compared to the High STD Risk condition. See Table $3 b$ for a full list of comparisons.

No significant differences in median Raw AUC were detected between the Low Attraction condition and the Low STD Risk condition $(p=.149)$. Additionally, no significant differences in median Raw AUC were detected between the Low Attraction condition and the High Pregnancy Risk condition $(p=.422)$. Lastly, no significant differences in median Raw AUC were detected between the Low Attraction condition and the Low Pregnancy Risk condition $(p=.836)$. See Table $3 b$ for a full list of comparisons. 
High STD Risk Raw AUC. The median AUC for the High STD Risk Raw AUC (Mdn = $1.00, \mathrm{IQR}=.00)$ differed from the median High Attraction Raw AUC $(\mathrm{Mdn}=.78, \mathrm{IQR}=.69 ; \mathrm{Z}$ $=-3.73, p<.001)$. Participants less steeply discounted condoms in the High STD Risk condition compared to the High Attraction condition. This suggests more preference for using a condom as the delay increased in the High STD Risk condition compared to the High Attraction condition. See Table $3 b$ for a full list of comparisons.

The median AUC for High STD Risk Raw AUC (Mdn = 1.00, IQR = .00) differed from the median the Low Attraction Raw AUC (Mdn = 1.00, IQR = .19; $\mathrm{Z}=-2.28, p=.023)$. Participants less steeply discounted condoms in the High STD Risk condition compared to the Low Attraction condition. This suggests more preference for using a condom as the delay increased in the High STD Risk condition compared to the Low Attraction condition. See Table $3 b$ for a full list of comparisons.

The median AUC for the High STD Risk Raw AUC (Mdn $=1.00, \mathrm{IQR}=.00)$ differed from the median High Pregnancy Risk Raw AUC (Mdn = 1.00, IQR = .12; Z = -2.07, $p=.038)$. Participants less steeply discounted condoms in the High STD Risk condition compared to the High Pregnancy Risk condition. This suggests more preference for using a condom as the delay increased in the High STD Risk condition compared to the High Pregnancy Risk condition. See Table $3 b$ for a full list of comparisons.

The median AUC for the High STD Risk Raw AUC (Mdn = 1.00, IQR =.00) differed from the median Low Pregnancy Risk Raw AUC $(\mathrm{Mdn}=1.00, \mathrm{IQR}=.22 ; \mathrm{Z}=-2.55, p=.011)$. Participants less steeply discounted condoms in the High STD Risk condition compared to the Low Pregnancy Risk condition. This suggests more preference for using a condom as the delay 
increased in the High STD Risk condition compared to the Low Pregnancy Risk condition. See Table $3 b$ for a full list of comparisons.

Low STD Risk Raw AUC. The median AUC for the Low STD Risk Raw AUC (Mdn = $.92, \mathrm{IQR}=.40)$ differed from the median High Pregnancy Risk Raw AUC $(\mathrm{Mdn}=1.00, \mathrm{IQR}=$ $.12 ; \mathrm{Z}=-2.48, p=.013)$. Participants more steeply discounted condoms in the Low STD Risk condition compared to the High Pregnancy Risk condition. This suggests less preference for using a condom as the delay increased in the Low STD Risk condition compared to the High Pregnancy Risk condition. See Table $3 b$ for a full list of comparisons.

The median AUC for the Low STD Risk Raw AUC (Mdn = .92, IQR = .40) differed from the median Low Pregnancy Risk Raw AUC $(\mathrm{Mdn}=1.00, \mathrm{IQR}=.22 ; \mathrm{Z}=-2.10, p=.035)$. Participants more steeply discounted condoms in the Low STD Risk condition compared to the Low Pregnancy Risk condition. This suggests less preference for using a condom as the delay increased in the Low STD Risk condition compared to the Low Pregnancy Risk condition. See Table $3 b$ for a full list of comparisons.

No significant differences in median Raw AUC were detected between Low STD Risk condition and the High Attraction condition $(p=.117)$. Additionally, no significant differences in median Raw AUC were detected between Low STD Risk condition and the Low Attraction condition $(p=.149)$. See Table $3 b$ for a full list of comparisons.

High Pregnancy Risk Raw AUC. The median AUC for the High Pregnancy Risk Raw AUC $(\mathrm{Mdn}=1.00, \mathrm{IQR}=.12)$ differed from the median High Attraction Raw AUC $(\mathrm{Mdn}=.78$, $\mathrm{IQR}=.69 ; \mathrm{Z}=-3.25, p<.001)$. Participants more less steeply discounted condoms in the High Pregnancy Risk condition compared to the High Attraction condition. This suggests more 
preference for using a condom as the delay increased in the High Pregnancy Risk condition compared to the High Attraction condition. See Table $3 b$ for a full list of comparisons.

The median AUC for the High Pregnancy Risk Raw AUC (Mdn = 1.00, IQR = .12) differed from the median High STD Risk Raw AUC $(\mathrm{Mdn}=1.00, \mathrm{IQR}=.00 ; \mathrm{Z}=-2.07, p=$ .038). Participants less steeply discounted condoms in the High Pregnancy Risk condition compared to the High STD Risk condition. This suggests more preference for using a condom as the delay increased in the High Pregnancy Risk condition compared to the High STD Risk condition. See Table $3 b$ for a full list of comparisons.

The median AUC for the High Pregnancy Risk Raw AUC (Mdn = 1.00, IQR = .12) differed from the median Low STD Risk Raw AUC $(\mathrm{Mdn}=.92, \mathrm{IQR}=.40 ; \mathrm{Z}=-2.48, p=.013)$. Participants less steeply discounted condoms in the High Pregnancy Risk condition compared to the Low STD Risk condition. This suggests more preference for using a condom as the delay increased in the High Pregnancy Risk condition compared to the Low STD Risk condition. See Table $3 b$ for a full list of comparisons.

No significant differences in median AUC were detected between the High Pregnancy Risk condition and the Attraction Low condition $(p=.422)$. See Table $3 b$ for a full list of comparisons.

Low Pregnancy Risk Raw AUC. The median AUC for the Low Pregnancy Risk Raw AUC $(\mathrm{Mdn}=1.00, \mathrm{IQR}=.22)$ differed from the median High Attraction Raw AUC $(\mathrm{Mdn}=.78$, $\mathrm{IQR}=.69 ; \mathrm{Z}=-3.49, p<.001)$. Participants less steeply discounted condoms in the Low Pregnancy Risk compared to the High Attraction condition. This suggests more preference for using a condom as the delay increased in the Low Pregnancy Risk condition compared to the High Attraction condition. See Table $3 \mathrm{~b}$ for a full list of comparisons. 
The median AUC for the Low Pregnancy Risk Raw AUC $(\mathrm{Mdn}=1.00, \mathrm{IQR}=.22)$ differed from the median High STD Risk Raw AUC $(\mathrm{Mdn}=1.00, \mathrm{IQR}=.00 ; \mathrm{Z}=-2.55, p=$ .011). Participants more steeply discounted condoms in the Low Pregnancy Risk condition compared to the High STD Risk condition. This suggests less preference for using a condom as the delay increased in the Low Pregnancy Risk condition compared to the High STD Risk condition. See Table $3 b$ for a full list of comparisons.

The median AUC for the Low Pregnancy Risk Raw AUC $(\mathrm{Mdn}=1.00, \mathrm{IQR}=.22)$ differed from the median Low STD Risk Raw AUC $(\mathrm{Mdn}=.92, \mathrm{IQR}=.40 ; \mathrm{Z}=-2.10, p=.035)$. Participants less steeply discounted condoms in the Low Pregnancy Risk condition compared to the Low STD Risk condition. This suggests more preference for using a condom as the delay increased in the Low Pregnancy Risk condition compared to the Low STD Risk condition. See Table $3 b$ for a full list of comparisons.

No significant differences in median AUC were detected between the Low Pregnancy Risk condition and the Low Attraction condition $(p=.836)$. See Table $3 b$ for a full list of comparisons.

To test Research Question 1b., a series of Wilcoxon Signed-rank Tests was computed between "Immediately Available" Condom Preferences for each Sexual Discounting Task condition. See Table $3 \mathrm{~b}$ for a full list of comparisons.

High Attraction “Immediately Available” Condom Preference. The median "Immediately Available" Condom Preference for the High Attraction condition (Mdn = $100.00 \%, \mathrm{IQR}=13.0)$ differed from the median "Immediately Available" Condom Preference for the High STD Risk condition $(\mathrm{Mdn}=100.00 \%, \mathrm{IQR}=.00 ; \mathrm{Z}=-2.67, p=.008)$. Participants 
responded with lower preference for an "immediately available" condom in the High Attraction condition compared to the High STD Risk condition. See Table $3 b$ for a full list of comparisons.

The median "Immediately Available" Condom Preference for the High Attraction condition $(\mathrm{Mdn}=100.00 \%, \mathrm{IQR}=13.0)$ differed from the median "Immediately Available" Condom Preference for the High Pregnancy Risk condition $(\mathrm{Mdn}=100.00 \%, \mathrm{IQR}=.00 ; \mathrm{Z}=-$ $2.40, p=.017)$. Participants responded with lower preference for an "immediately available" condom in the High Attraction condition compared to the High Pregnancy Risk condition. See Table $3 b$ for a full list of comparisons.

No significant differences in median "Immediately Available" Condom Preference were detected between the High Attraction condition and the Low STD Risk condition $(p=.308)$. Additionally, no significant differences in median "Immediately Available" Condom Preference were detected between the High Attraction condition and the Low Pregnancy Risk condition $(p=$ .074). See Table $3 b$ for a full list of comparisons.

Low Attraction "Immediately Available” Condom Preference. No significant differences in median "Immediately Available" Condom Preference were detected between the Low Attraction condition and the High STD Risk condition $(p=.173)$. Additionally, no significant differences in median "Immediately Available" Condom Preference were detected between the Low Attraction condition and the Low STD Risk condition $(p=.463)$. Similarly, no significant differences in median "Immediately Available" Condom Preference were detected between the Low Attraction condition and the High Pregnancy Risk condition $(p=.345)$. Lastly, no significant differences in median "Immediately Available" Condom Preference were detected between the Low Attraction condition and the Low Pregnancy Risk condition $(p=.893)$. See Table $3 \mathrm{~b}$ for a full list of comparisons. 
High STD Risk "Immediately Available" Condom Preference. The median

"Immediately Available" Condom Preference for the High STD Risk condition (Mdn = $100.00 \%, \mathrm{IQR}=.00)$ differed from the median "Immediately Available" Condom Preference for the High Attraction condition $(\mathrm{Mdn}=100.00 \%, \mathrm{IQR}=13.0 ; \mathrm{Z}=-2.67, p=.008)$. Participants responded with higher preference for an "immediately available" condom in the High STD Risk condition compared to the High Attraction condition. See Table $3 b$ for a full list of comparisons.

No significant differences in median "Immediately Available" Condom Preference were detected between the High STD Risk condition and the Low Attraction condition $(p=.173)$. Additionally, no significant differences in median "Immediately Available" Condom Preference were detected between the High STD Risk condition and the High Pregnancy Risk condition $(p=$ .465). Lastly, no significant differences in median "Immediately Available" Condom Preference were detected between the High STD Risk condition and the Low Pregnancy Risk condition $(p=$ .150). See Table $3 b$ for a full list of comparisons.

Low STD Risk “Immediately Available” Condom Preference. No significant differences in median "Immediately Available" Condom Preference were detected between the Low STD Risk condition and the High Attraction condition $(p=.308)$. Additionally, no significant differences in median "Immediately Available" Condom Preference were detected between the Low STD Risk condition and the Low Attraction condition $(p=.463)$. Similarly, no significant differences in median "Immediately Available" Condom Preference were detected between the Low STD Risk condition and the High Pregnancy Risk condition $(p=.173)$. Similarly, no significant differences in median "Immediately Available" Condom Preference were detected between the Low STD Risk condition and the Low Pregnancy Risk condition $(p=.624)$. See Table $3 \mathrm{~b}$ for a full list of comparisons. 
High Pregnancy Risk “Immediately Available” Condom Preference. The median "Immediately Available" Condom Preference for the High Pregnancy Risk condition (Mdn = $100.00 \%, \mathrm{IQR}=.00)$ differed from the median "Immediately Available" Condom Preference for the High Attraction condition $(\mathrm{Mdn}=100.00 \%, \mathrm{IQR}=13.0 ; \mathrm{Z}=-2.40, p=.017)$. Participants responded with higher preference for an "immediately available" condom in the High Pregnancy Risk condition compared to the High Attraction condition. See Table $3 b$ for a full list of comparisons.

No significant differences in median "Immediately Available" Condom Preference were detected between the High Pregnancy Risk and the Low Attraction conditions $(p=.345)$. Additionally, no significant differences in median "Immediately Available" Condom Preference were detected between the High Pregnancy Risk and the High STD Risk conditions $(p=.465)$. Lastly, no significant differences in median "Immediately Available" Condom Preference were detected between the High Pregnancy Risk and the Low STD Risk conditions $(p=.173)$. See Table $3 b$ for a full list of comparisons.

Low Pregnancy Risk “Immediately Available” Condom Preference. No significant differences in median "Immediately Available" Condom Preference were detected between the Low Pregnancy Risk and the High Attraction condition $(p=.075)$. Additionally, no significant differences in median "Immediately Available" Condom Preference were detected between the Low Pregnancy Risk and the Low Attraction condition $(p=.893)$. Similarly, no significant differences in median "Immediately Available" Condom Preference were detected between the Low Pregnancy Risk condition and the High STD Risk condition $(p=.150)$. Lastly, no significant differences in median "Immediately Available" Condom Preference were detected 
between the Low Pregnancy Risk condition and the Low STD Risk condition $(p=.624)$. See Table $3 b$ for a full list of comparisons.

To test Research Question 1c., a series of Wilcoxon Signed-rank Tests was computed between each Standardized AUC Sexual Discounting Task condition. See Table $3 \mathrm{~b}$ for a full list of comparisons.

High Attraction Standardized AUC. The median AUC for the High Attraction Standardized AUC $(\mathrm{Mdn}=.85, \mathrm{IQR}=.43)$ differed from the median High STD Risk Standardized AUC $(\mathrm{Mdn}=1.00, \mathrm{IQR}=.00 ; \mathrm{Z}=-3.54, p<.001)$. Participants more steeply discounted condoms in the High Attraction compared to the High STD Risk condition. This suggests less preference for using a condom as the delay increased in the High Attraction condition compared to the High STD Risk condition. See Table $3 b$ for a full list of comparisons.

The median AUC for the High Attraction Standardized AUC $(\mathrm{Mdn}=.85, \mathrm{IQR}=.43)$ differed from the median Low STD Risk Standardized AUC $(\mathrm{Mdn}=1.00, \mathrm{IQR}=.17 ; \mathrm{Z}=-2.07$, $p=.039)$. Participants more steeply discounted condoms in the High Attraction compared to the Low STD Risk condition. This suggests less preference for using a condom as the delay increased in the High Attraction condition compared to the Low STD Risk condition. See Table $3 b$ for a full list of comparisons.

The median AUC for the High Attraction Standardized AUC $(\mathrm{Mdn}=.85, \mathrm{IQR}=.43)$ differed from the median High Pregnancy Risk Standardized AUC $(\mathrm{Mdn}=1.00, \mathrm{IQR}=.03 ; \mathrm{Z}=$ -3.02, $p=.002)$. Participants more steeply discounted condoms in the High Attraction compared to the High Pregnancy Risk condition. This suggests less preference for using a condom as the delay increased in the High Attraction condition compared to the High Pregnancy Risk condition. See Table $3 \mathrm{~b}$ for a full list of comparisons. 
The median AUC for the High Attraction Standardized AUC $(\mathrm{Mdn}=.85, \mathrm{IQR}=.43)$ differed from the median Low Pregnancy Risk Standardized AUC (Mdn $=1.00, \mathrm{IQR}=.06 ; \mathrm{Z}=$ 3.58, $p<.001)$. Participants more steeply discounted condoms in the High Attraction compared to the Low Pregnancy Risk condition. This suggests less preference for using a condom as the delay increased in the High Attraction condition compared to the Low Pregnancy Risk condition. See Table $3 b$ for a full list of comparisons.

Low Attraction Standardized AUC. The median AUC for the Low Attraction Standardized AUC $(\mathrm{Mdn}=1.00, \mathrm{IQR}=.01)$ differed from the median High STD Risk Standardized AUC $(\mathrm{Mdn}=1.00, \mathrm{IQR}=.00 ; \mathrm{Z}=-1.96, p=.050)$. Participants more steeply discounted condoms in the Low Attraction compared to the High STD Risk condition. This suggests less preference for using a condom as the delay increased in the Low Attraction condition compared to the High STD Risk condition. See Table $3 b$ for a full list of comparisons.

No significant differences in median Standardized AUC were detected between the Low Attraction condition and the Low STD Risk condition $(p=.326)$. Additionally, no significant differences in median Standardized AUC were detected between the Low Attraction condition and the High Pregnancy Risk condition $(p=.695)$. Lastly, no significant differences in median Standardized AUC were detected between the Low Attraction condition and the Low Pregnancy Risk condition $(p=.955)$. See Table $3 b$ for a full list of comparisons.

High STD Risk Standardized AUC. The median AUC for the High STD Risk Standardized AUC $(\mathrm{Mdn}=1.00, \mathrm{IQR}=.00)$ differed from the median High Attraction Standardized AUC $(\mathrm{Mdn}=.85, \mathrm{IQR}=.43 ; \mathrm{Z}=-3.54, p<.001)$. Participants less steeply discounted condoms in the High STD Risk condition compared to the High Attraction condition. 
This suggests more preference for using a condom as the delay increased in the High STD Risk condition compared to the High Attraction condition. See Table $3 b$ for a full list of comparisons.

The median AUC for High STD Risk Standardized AUC (Mdn = 1.00, IQR =.00) differed from the median the Low Attraction Standardized AUC $(\mathrm{Mdn}=1.00, \mathrm{IQR}=.01 ; \mathrm{Z}=$ $1.96, p=.050)$. Participants less steeply discounted condoms in the High STD Risk condition compared to the Low Attraction condition. This suggests more preference for using a condom as the delay increased in the High STD Risk condition compared to the Low Attraction condition. See Table $3 b$ for a full list of comparisons.

The median AUC for the High STD Risk Standardized AUC (Mdn = 1.00, IQR = .00) differed from the median Low Pregnancy Risk Standardized AUC (Mdn = 1.00, IQR = .06; Z = $2.09, p=.037)$. Participants less steeply discounted condoms in the High STD Risk condition compared to the Low Pregnancy Risk condition. This suggests more preference for using a condom as the delay increased in the High STD Risk condition compared to the Low Pregnancy Risk condition. See Table $3 b$ for a full list of comparisons.

No significant differences in median Standardized AUC were detected between the High STD Risk condition and the High Pregnancy Risk condition $(p=.069)$. See Table $3 b$ for a full list of comparisons.

Low STD Risk Standardized AUC. The median AUC for the Low STD Risk Standardized AUC $(\mathrm{Mdn}=1.00, \mathrm{IQR}=.17)$ differed from the median High Attraction Standardized AUC $(\mathrm{Mdn}=.85, \mathrm{IQR}=.43 ; \mathrm{Z}=-2.07, p=.039)$. Participants more steeply discounted condoms in the Low STD Risk condition compared to the High Attraction condition. This suggests less preference for using a condom as the delay increased in the Low STD Risk condition compared to the High Attraction condition. See Table $3 \mathrm{~b}$ for a full list of comparisons. 
The median AUC for the Low STD Risk Standardized AUC $(\mathrm{Mdn}=1.00, \mathrm{IQR}=.17)$ differed from the median Low Pregnancy Risk Standardized AUC (Mdn = 1.00, IQR = .06; Z = $2.51, p=.012)$. Participants more steeply discounted condoms in the Low STD Risk condition compared to the Low Pregnancy Risk condition. This suggests less preference for using a condom as the delay increased in the Low STD Risk condition compared to the Low Pregnancy Risk condition. See Table $3 b$ for a full list of comparisons.

No significant differences in median Standardized AUC were detected between Low STD Risk condition and the Low Attraction condition $(p=.326)$. Additionally, no significant differences in median Standardized AUC were detected between Low STD Risk condition and the High Pregnancy Risk condition $(p=.053)$. See Table $3 b$ for a full list of comparisons.

High Pregnancy Risk Standardized AUC. The median AUC for the High Pregnancy Risk Raw AUC $(\mathrm{Mdn}=1.00, \mathrm{IQR}=.03)$ differed from the median High Attraction Raw AUC $(\mathrm{Mdn}=.85 ; \mathrm{IQR}=.43 ; \mathrm{Z}=-3.02, p=.002)$. Participants more less steeply discounted condoms in the High Pregnancy Risk condition compared to the High Attraction condition. This suggests more preference for using a condom as the delay increased in the High Pregnancy Risk condition compared to the High Attraction condition. See Table $3 b$ for a full list of comparisons.

No significant differences in median AUC were detected between the High Pregnancy Risk condition and the Attraction Low condition $(p=.695)$. Additionally, no significant differences in median AUC were detected between the High Pregnancy Risk condition and the High STD Risk condition $(p=.069)$. Lastly, no significant differences in median AUC were detected between the High Pregnancy Risk condition and the Low STD Risk condition ( $p=$ .053). See Table $3 b$ for a full list of comparisons. 
Low Pregnancy Risk Standardized AUC. The median AUC for the Low Pregnancy Risk Raw AUC $(\mathrm{Mdn}=1.00, \mathrm{IQR}=.06)$ differed from the median High Attraction Raw AUC $(\mathrm{Mdn}=$ $.85, \mathrm{IQR}=.43 ; \mathrm{Z}=-3.58, p<.001)$. Participants less steeply discounted condoms in the Low Pregnancy Risk compared to the High Attraction condition. This suggests more preference for using a condom as the delay increased in the Low Pregnancy Risk condition compared to the High Attraction condition. See Table $3 b$ for a full list of comparisons.

The median AUC for the Low Pregnancy Risk Raw AUC $(\mathrm{Mdn}=1.00, \mathrm{IQR}=.06)$ differed from the median High STD Risk Raw AUC $(\mathrm{Mdn}=1.00, \mathrm{IQR}=.00 ; \mathrm{Z}=-2.09, p=$ .037). Participants more steeply discounted condoms in the Low Pregnancy Risk condition compared to the High STD Risk condition. This suggests less preference for using a condom as the delay increased in the Low Pregnancy Risk condition compared to the High STD Risk condition. See Table $3 b$ for a full list of comparisons.

The median AUC for the Low Pregnancy Risk Raw AUC $(\mathrm{Mdn}=1.00, \mathrm{IQR}=.06)$ differed from the median Low STD Risk Raw AUC $(\mathrm{Mdn}=1.00, \mathrm{IQR}=.17 ; \mathrm{Z}=-2.51, p=$ .012). Participants less steeply discounted condoms in the Low Pregnancy Risk condition compared to the Low STD Risk condition. This suggests more preference for using a condom as the delay increased in the Low Pregnancy Risk condition compared to the Low STD Risk condition. See Table $3 b$ for a full list of comparisons.

No significant differences in median AUC were detected between the Low Pregnancy Risk condition and the Low Attraction condition $(p=.955)$. See Table $3 b$ for a full list of comparisons.

Research Question 2. Does reward magnitude influence preference for monetary and sexual discounting was broken into three components for analysis, Raw AUC, "Immediately 
Available" condom preferences, and Standardized AUC. To test Research Question 2a., a series of Wilcoxon Signed-rank Tests was computed between Raw AUCs within the Monetary DelayDiscounting Task. To test Research Question 2b., a series of Wilcoxon Signed-rank Tests was computed between Raw AUCs within each Sexual Discounting Task condition. To test Research Question 2c., a series of Wilcoxon Signed-rank Tests was computed between "Immediately Available" Condom Preferences within each Sexual Discounting Task condition. See Table 4 for full list of comparisons. To test Research Question 2d., a series of Wilcoxon Signed-rank Tests was computed between Standardized AUCs within each Sexual Discounting Task condition. See Table $4 \mathrm{~b}$ for full list of comparisons.

\section{RQ2a: Within Condition Comparison for Monetary Delay-Discounting Task AUC.}

In order to examine magnitude differences in delay discounting, comparisons were computed within the Monetary Delay-Discounting Task between $\$ 10$ and $\$ 100$ conditions. The median AUC for the $\$ 10$ Monetary condition $(\mathrm{Mdn}=.36, \mathrm{IQR}=.82)$ differed from the median AUC for the $\$ 100$ Monetary condition $(\mathrm{Mdn}=.70, \mathrm{IQR}=.69 ; \mathrm{Z}=-2.22, p=.026)$. Participants more steeply discounted money in the $\$ 10$ Monetary condition compared to the $\$ 100$ Monetary condition. This indicates a greater preference for smaller sooner rewards in the $\$ 10$ Monetary condition compared to the $\$ 100$ Monetary condition. See Figure $1 \mathrm{~b}$ for visual depiction of $\$ 10$ and \$100 Monetary percent of immediate value chosen across delays. See Table $4 \mathrm{~b}$ for full list of comparisons. Figure 3b (\$10) and 4b (\$100) illustrate individual trajectories for each of the 27 participants with complete data.

\section{RQ2b: Within Condition Comparison for All Raw Sexual Discounting Task AUC.} In order to examine magnitude differences in delay discounting, comparisons were computed 
between Raw AUCs within each Sexual Discounting Task condition. See Table 4b for full list of comparisons.

Attraction Raw AUC. The median Raw AUC for the High Attraction condition (Mdn = $.78, \mathrm{IQR}=.69)$ differed from the median Raw AUC for the Low Attraction condition $(\mathrm{Mdn}=$ $1.00, \mathrm{IQR}=.19 ; \mathrm{Z}=-2.66, p=.008)$. Participants more steeply discounted condom use in the High Attraction condition compared to the Low Attraction condition. This indicates less preference for a delayed condom in the High Attraction condition compared to the Low Attraction condition. See Table $4 b$ for full list of comparisons. See Figure $2 b$ for a visual depiction of percent likelihood of condom use by delay in each condition. Figure 5b (High Attractiveness) and 6b (Low Attractiveness) illustrate individual trajectories for each of the 27 participants with complete data.

STD Risk Raw AUC. The median Raw AUC for the High STD Risk condition (Mdn = $1.00, \mathrm{IQR}=.00)$ differed from the median Raw AUC for the Low STD Risk condition $(\mathrm{Mdn}=$ $.92, \mathrm{IQR}=.40 ; \mathrm{Z}=-3.41, p=.001)$. Participants less steeply discounted condom use in the High STD Risk condition compared to the Low STD Risk condition. This indicates more preference for a delayed condom in the High STD Risk condition compared to the Low STD Risk condition. See Table $4 b$ for full list of comparisons. See Figure $2 b$ for a visual depiction of percent likelihood of condom use by delay in each condition. Figure 7b (High STD Risk) and 8b (Low STD Risk) illustrate individual trajectories for each of the 27 participants with complete data.

Pregnancy Risk Raw AUC. The median Raw AUC for the High Pregnancy Risk condition $(\mathrm{Mdn}=1.00, \mathrm{IQR}=.12)$ did not significantly differ from the median Raw AUC for the Low Pregnancy Risk condition $(\mathrm{Mdn}=1.00, \mathrm{IQR}=.22 ; p=.173)$. See Table $4 \mathrm{~b}$ for full list of 
comparisons. See Figure $2 \mathrm{~b}$ for a visual depiction of percent likelihood of condom use by delay in each condition.

To test Research Question 2c., a series of Wilcoxon Signed-rank Tests was computed between "Immediately Available" Condom Preferences within each Sexual Discounting Task condition. See Table $6 \mathrm{~b}$ for full list of comparisons.

Attraction "Immediately Available" Condom Preferences. The median "Immediately Available" Condom Preference for the High Attraction condition $(\mathrm{Mdn}=100.00 \%, \mathrm{IQR}=13.0)$ differed from the "Immediately Available" Condom Preference for the Low Attraction condition $(\mathrm{Mdn}=100.00 \%, \mathrm{IQR}=.00 ; \mathrm{Z}=-2.29, p=.022)$. Participants responded with lower preference for an "immediately available" condom in the High Attraction condition compared to the Low Attraction condition. See Table $6 \mathrm{~b}$ for full list of comparisons.

STD Risk “Immediately Available” Condom Preferences. The median "Immediately Available" Condom Preference for the High STD Risk condition $(\mathrm{Mdn}=100.00 \%, \mathrm{IQR}=.00)$ was not significantly different from the "Immediately Available" Condom Preference for the Low STD Risk condition $(\mathrm{Mdn}=100.00 \%, \mathrm{IQR}=.00 ; \mathrm{Z}=-1.90, p=.058)$. See Table $6 \mathrm{~b}$ for full list of comparisons.

Pregnancy Risk “Immediately Available” Condom Preferences. The median “Immediately Available” Condom Preference for the High Pregnancy Risk condition (Mdn = $100.00 \%, \mathrm{IQR}=.00$ ) was not significantly different from the "Immediately Available" Condom Preference for the Low Pregnancy Risk condition $(\mathrm{Mdn}=100.00 \%, \mathrm{IQR}=.00 ; \mathrm{Z}=-1.15, p=$ .249). See Table $6 \mathrm{~b}$ for full list of comparisons.

\section{RQ2d: Within Condition Comparison for All Standardized Sexual Discounting}

Task AUC. In order to examine magnitude differences in delay discounting, comparisons were 
computed between Standardized AUCs within each Sexual Discounting Task condition. See Table $5 \mathrm{~b}$ for full list of comparisons.

Attraction Standardized AUC. The median Standardized AUC for the High Attraction condition $(\mathrm{Mdn}=.85, \mathrm{IQR}=.43)$ differed from the median Standardized AUC for the Low Attraction condition $(\mathrm{Mdn}=1.00, \mathrm{IQR}=.01 ; \mathrm{Z}=-2.43, p=.015)$. Participants more steeply discounted condom use in the High Attraction condition compared to the Low Attraction condition. This indicates less preference for a delayed condom in the High Attraction condition compared to the Low Attraction condition. See Table $5 b$ for full list of comparisons.

STD Risk Standardized AUC. The median Standardized AUC for the High STD Risk condition $(\mathrm{Mdn}=1.00, \mathrm{IQR}=.00)$ differed from the median Standardized AUC for the Low STD Risk condition $(\mathrm{Mdn}=1.00, \mathrm{IQR}=.17 ; \mathrm{Z}=-2.97, p=.003)$. Participants less steeply discounted condom use in the High STD Risk condition compared to the Low STD Risk condition. This indicates more preference for a delayed condom in the High STD Risk condition compared to the Low STD Risk condition. See Table 5b for full list of comparisons.

Pregnancy Risk Standardized AUC. The median Standardized AUC for the High Pregnancy Risk condition $(\mathrm{Mdn}=1.00, \mathrm{IQR}=.03)$ did not significantly differ from the median Standardized AUC for the Low Pregnancy Risk condition $(\mathrm{Mdn}=1.00, \mathrm{IQR}=.06 ; p=1.00)$. See Table $5 \mathrm{~b}$ for full list of comparisons.

Research Question 3. Are differences in delay discounting associated with individual differences? was broken into two different analysis, Spearman correlations and Mann-Whitney U tests, and three components for analysis, Raw AUC, "Immediately Available” condom preferences, and Standardized AUC. 
To test Research Question 3a, Spearman rank correlations were computed using Future Time Perspective, MCAS Condom Attitudes, sexual arousal and Raw AUC for each Sexual Discounting Task condition. AUC is on a 0-1 scale whereby lower AUC indicates less preference for condoms across delays. See Table $4 \mathrm{~b}$ for the full list of comparisons.

Future Time Perspective. Future Time Perspective was categorized into three subfactors: Future as Open, Future as Limited, and Future as Ambiguous (Brothers et al., 2014).

Future as Open. No significant correlations were observed between Future as Open and Raw AUC across the Sexual Discounting Task conditions: High Attraction, Low Attraction, High STD Risk, Low STD Risk, High Pregnancy Risk, or Low Pregnancy Risk.

Future as Limited. No significant correlations were observed between Future as Limited and Raw AUC across the Sexual Discounting Task conditions: High Attraction, Low Attraction, High STD Risk, Low STD Risk, High Pregnancy Risk, or Low Pregnancy Risk.

Future as Ambiguous. No significant correlations were observed between Future as Limited and Raw AUC across the Sexual Discounting Task conditions: High Attraction, Low Attraction, High STD Risk, Low STD Risk, High Pregnancy Risk, or Low Pregnancy Risk. MCAS. The UCLA Multidimensional Condom Attitude Scale (MCAS) was categorized into three subfactors (Helweg-Larsen \& Collins, 1994).

MCAS Condom Pleasure. No significant correlations were observed between MCAS Condom Pleasure and Raw AUC across the Sexual Discounting Task conditions: High Attraction, Low Attraction, High STD Risk, Low STD Risk, High Pregnancy Risk, or Low Pregnancy Risk.

MCAS Identity Stigma Related to Condom Use. No significant correlations were observed between MCAS Identity Stigma Related to Condom Use and Raw AUC across the Sexual 
Discounting Task conditions: High Attraction, Low Attraction, High STD Risk, Low STD Risk, High Pregnancy Risk, or Low Pregnancy Risk.

MCAS Embarrassment About Negotiation and Use of Condoms. No significant correlations were observed between MCAS Embarrassment About Negotiation and Use of Condoms and Raw AUC across the Sexual Discounting Task conditions: High Attraction, Low Attraction, High STD Risk, Low STD Risk, High Pregnancy Risk, or Low Pregnancy Risk.

Sexual Arousal. No significant correlations were observed between sexual arousal and Raw AUC across the Sexual Discounting Task conditions: High Attraction, Low Attraction, High STD Risk, Low STD Risk, High Pregnancy Risk, or Low Pregnancy Risk.

To test Research Question 3b., a Mann-Whitney U was used due to non-parametric data to test median differences in Raw AUC across gender for each the Sexual Discounting Task condition (Gender X Attractiveness, Gender X STD Risk, Gender X Pregnancy Risk). See Table $7 \mathrm{~b}$ for the full list of gender comparisons.

Gender. No significant differences were observed between gender and Raw AUC across the Sexual Discounting Task conditions High Attraction, Low Attraction, High STD Risk, Low STD Risk, High Pregnancy Risk, or Low Pregnancy Risk.

To test Research Question 3c, Spearman rank correlations were computed using Future Time Perspective, MCAS Condom Attitudes, sexual arousal and "Immediately Available" Condom Preferences for each Sexual Discounting Task condition. Higher "Immediately Available" Condom Preferences indicate higher likelihood of using a condom during the 0-delay condition. See Table $6 b$ for the full list of comparisons.

Future Time Perspective. Future Time Perspective was categorized into three subfactors: Future as Open, Future as Limited, and Future as Ambiguous (Brothers et al., 2014). 
Future as Open. No significant correlations were observed between Future as Open and "Immediately Available" Condom Preferences across the Sexual Discounting Task conditions: High Attraction, Low Attraction, High STD Risk, Low STD Risk, High Pregnancy Risk, or Low Pregnancy Risk.

Future as Limited. No significant correlations were observed between Future as Limited and "Immediately Available" Condom Preferences across the Sexual Discounting Task conditions: High Attraction, Low Attraction, High STD Risk, Low STD Risk, High Pregnancy Risk, or Low Pregnancy Risk.

Future as Ambiguous. No significant correlations were observed between Future as Ambiguous and "Immediately Available" Condom Preferences across the Sexual Discounting Task conditions: High Attraction, Low Attraction, High STD Risk, Low STD Risk, High Pregnancy Risk, or Low Pregnancy Risk.

MCAS. The UCLA Multidimensional Condom Attitude Scale (MCAS) was categorized into three subfactors (Helweg-Larsen \& Collins, 1994).

MCAS Condom Pleasure. No significant correlations were observed between MCAS Condom Pleasure and "Immediately Available" Condom Preferences across the Sexual Discounting Task conditions: High Attraction, Low Attraction, High STD Risk, Low STD Risk, High Pregnancy Risk, or Low Pregnancy Risk.

MCAS Identity Stigma Related to Condom Use. No significant correlations were observed between MCAS Identity Stigma Related to Condom Use and "Immediately Available" Condom Preferences across the Sexual Discounting Task conditions: High Attraction, Low Attraction, High STD Risk, Low STD Risk, High Pregnancy Risk, or Low Pregnancy Risk. 
MCAS Embarrassment About Negotiation and Use of Condoms. MCAS Embarrassment About Negotiation and Use of Condoms was significantly positively correlated with High Pregnancy Risk "Immediately Available" Condom Preferences, $\mathrm{r}_{\mathrm{s}}(25)=.395, p<.05$. That is, a higher preference for an "Immediately Available" condom in High Pregnancy Risk condition was associated with a higher endorsement of the MCAS Embarrassment About Negotiation and Use of Condoms. Conversely, a lower preference for an "Immediately Available" condom in the High Pregnancy Risk condition was associated with a lower endorsement of the MCAS Embarrassment About Negotiation and Use of Condoms.

No significant correlations were observed between MCAS Embarrassment About Negotiation and Use of Condoms and "Immediately Available" Condom Preferences across the Sexual Discounting Task conditions: High Attraction, Low Attraction, High STD Risk, Low STD Risk, or Low Pregnancy Risk.

Sexual Arousal. No significant correlations were observed between Sexual Arousal and "Immediately Available" Condom Preferences across the Sexual Discounting Task conditions: High Attraction, Low Attraction, High STD Risk, Low STD Risk, High Pregnancy Risk, or Low Pregnancy Risk.

To test Research Question 3d., Mann-Whitney U were used due to non-parametric data to test median differences in "Immediately Available" Condom Preferences across gender for each the Sexual Discounting Task condition (Gender X Attractiveness, Gender X STD Risk, Gender X Pregnancy Risk). See Table 7b for the full list of gender comparisons.

Gender. No other significant differences were observed between gender and "Immediately Available" Condom Preferences across the Sexual Discounting Task conditions: 
High Attraction, Low Attraction, High STD Risk, Low STD Risk, High Pregnancy Risk, or Low Pregnancy Risk. See Table 10 for additional information.

To test Research Question 3e, Spearman rank correlations were computed using Future Time Perspective, MCAS Condom Attitudes, sexual arousal and Standardize AUC for each Sexual Discounting Task condition. Standardized AUC uses a participants 0-delay condom preference and divides subsequent timepoints by this value prior to calculating AUC. The standardization is used to isolate the effects of delay on condom preference. Standardized AUC is on a 0-1 scale whereby lower AUC indicates less preference for condoms across delays. See Table $5 b$ for the full list of comparisons.

Future Time Perspective. Future Time Perspective was categorized into three subfactors: Future as Open, Future as Limited, and Future as Ambiguous (Brothers et al., 2014).

Future as Open. No significant correlations were observed between Future as Open and Standardized AUC across the Sexual Discounting Task conditions: High Attraction, Low Attraction, High STD Risk, Low STD Risk, High Pregnancy Risk, or Low Pregnancy Risk.

Future as Limited. No significant correlations were observed between Future as Limited and Standardized AUC across the Sexual Discounting Task conditions: High Attraction, Low Attraction, High STD Risk, Low STD Risk, High Pregnancy Risk, or Low Pregnancy Risk. Future as Ambiguous. No significant correlations were observed between Future as Limited and Standardized AUC across the Sexual Discounting Task conditions: High Attraction, Low Attraction, High STD Risk, Low STD Risk, High Pregnancy Risk, or Low Pregnancy Risk. MCAS. The UCLA Multidimensional Condom Attitude Scale (MCAS) was categorized into three subfactors (Helweg-Larsen \& Collins, 1994). 
MCAS Condom Pleasure. No significant correlations were observed between MCAS Condom Pleasure and Standardized AUC across the Sexual Discounting Task conditions: High Attraction, Low Attraction, High STD Risk, Low STD Risk, High Pregnancy Risk, or Low Pregnancy Risk.

MCAS Identity Stigma Related to Condom Use. No significant correlations were observed between MCAS Identity Stigma Related to Condom Use and Standardized AUC across the Sexual Discounting Task conditions: High Attraction, Low Attraction, High STD Risk, Low STD Risk, High Pregnancy Risk, or Low Pregnancy Risk.

MCAS Embarrassment About Negotiation and Use of Condoms. No significant correlations were observed between MCAS Embarrassment About Negotiation and Use of Condoms and Standardized AUC across the Sexual Discounting Task conditions: High Attraction, Low Attraction, High STD Risk, Low STD Risk, High Pregnancy Risk, or Low Pregnancy Risk.

Sexual Arousal. No significant correlations were observed between sexual arousal and Standardized AUC across the Sexual Discounting Task conditions: High Attraction, Low Attraction, High STD Risk, Low STD Risk, High Pregnancy Risk, or Low Pregnancy Risk.

To test Research Question 3f., a Mann-Whitney U was used due to non-parametric data to test median differences in Standardized AUC across gender for each the Sexual Discounting Task condition (Gender X Attractiveness, Gender X STD Risk, Gender X Pregnancy Risk). See Table $7 \mathrm{~b}$ for the full list of gender comparisons.

Gender. No significant differences were observed between gender and Standardized AUC across the Sexual Discounting Task conditions High Attraction, Low Attraction, High STD Risk, Low STD Risk, High Pregnancy Risk, or Low Pregnancy Risk. 
Table 1b

Participant Characteristics

\begin{tabular}{|c|c|c|c|c|c|}
\hline & \multicolumn{2}{|c|}{$\begin{array}{c}\text { Enrolled Participants } \\
\quad(n=113)\end{array}$} & & & \\
\hline & $\begin{array}{c}\text { Incomplete } \\
\text { Discounting } \\
\text { Data } \\
(n=86)\end{array}$ & $\begin{array}{c}\text { Complete } \\
\text { Discounting } \\
\text { Data } \\
(n=27)\end{array}$ & & & \\
\hline Variable & \multicolumn{2}{|c|}{$M(S E)$} & $F$ & $d f$ & $p$ \\
\hline Age & $34.39(1.01)$ & $34.19(1.34)$ & 0.01 & 1,110 & .917 \\
\hline Education & $12.53(0.2)$ & $12.04(0.47)$ & 1.30 & 1,111 & .257 \\
\hline \multicolumn{6}{|l|}{ Future Time Perspective } \\
\hline Future as Open & $15.85(0.31)$ & $15.41(0.5)$ & 0.49 & 1,111 & .486 \\
\hline Future as Limited & $12.34(0.35)$ & $11.93(0.66)$ & 0.32 & 1,111 & .571 \\
\hline Future as Ambiguous & $11.9(0.4)$ & $11.48(0.64)$ & 0.27 & 1,111 & .605 \\
\hline \multicolumn{6}{|l|}{ MCAS } \\
\hline Pleasure & $3.15(0.12)$ & $3.54(0.21)$ & 2.54 & 1,110 & .114 \\
\hline Stigma & $5.53(0.14)$ & $6.03(0.17)$ & 3.63 & 1,111 & .060 \\
\hline Negotiation and Use & $5.31(0.14)$ & $5.79(0.19)$ & 3.16 & 1,110 & .078 \\
\hline \multirow[t]{2}{*}{ Sexual Arousal $(\%)$} & $46.78(3.89)$ & $40.85(6.11)$ & 0.59 & 1,111 & .446 \\
\hline & \multicolumn{2}{|c|}{$n(\%)$} & $\chi^{2}$ & $d f$ & $p$ \\
\hline Gender & & & .34 & 1 & .560 \\
\hline Men & $24(27.9)$ & $6(22.2)$ & & & \\
\hline Women & $62(72.1)$ & $21(77.8)$ & & & \\
\hline Race & & & 1.03 & 3 & .795 \\
\hline Non-White & $7(8.1)$ & $1(3.7)$ & & & \\
\hline White & 79 (91.9) & $26(96.3)$ & & & \\
\hline $\begin{array}{l}n . b . \text { MCAS = UCLA Multid } \\
\text { made between Incomplete D } \\
\text { values were imputed for the }\end{array}$ & $\begin{array}{l}\text { nsional Cor } \\
\text { unting Dat } \\
\text { ables in thi }\end{array}$ & $\begin{array}{l}\text { Attitude S } \\
\text { d Complete } \\
\text { le; } * p<.05 \text {; }\end{array}$ & $\begin{array}{l}\mathrm{e} ; \mathrm{C} \\
\mathrm{scou}\end{array}$ & $\overline{\text { ariso }}$ & $\begin{array}{l}\text { were } \\
\text { no } \\
1\end{array}$ \\
\hline
\end{tabular}


Table $2 b$

Descriptive Statistics for Raw AUC, Standardized AUC, and "Immediately Available” Condom Preferences $(n=27)$

\begin{tabular}{|c|c|c|c|c|c|c|}
\hline & $\begin{array}{l}\text { Raw } \\
\text { AUC }\end{array}$ & $\begin{array}{c}\text { Standardized } \\
\text { AUC }\end{array}$ & $\begin{array}{l}\text { "Immediately } \\
\text { Available" } \\
\text { Condom } \\
\text { Preferences } \\
(\%)\end{array}$ & $\begin{array}{l}\text { Raw } \\
\text { AUC }\end{array}$ & $\begin{array}{c}\text { Standardized } \\
\text { AUC }\end{array}$ & $\begin{array}{c}\text { "Immediately } \\
\text { Available" } \\
\text { Condom } \\
\text { Preferences } \\
(\%)\end{array}$ \\
\hline Variable & & Median (IRQ) & & & Mean (SE) & \\
\hline Attraction (High) & $.78(.69)$ & $.85(.43)$ & $100(13.0)$ & $.63(.07)$ & $.71(.07)$ & $91.30(2.79)$ \\
\hline Attraction (Low) & $1.00(.19)$ & $1.00(.01)$ & $100(.00)$ & $.86(.05)$ & $.89(.05)$ & $96.52(2.01)$ \\
\hline STD Risk (High) & $1.00(.00)$ & $1.00(.00)$ & $100(.00)$ & $.98(.02)$ & $.98(.02)$ & $99.63(0.33)$ \\
\hline STD Risk (Low) & $.92(.40)$ & $1.00(.17)$ & $100(.00)$ & $.77(.06)$ & $.85(.05)$ & $94.78(2.62)$ \\
\hline Pregnancy Risk (High) & $1.00(.12)$ & $1.00(.03)$ & $100(.00)$ & $.91(.03)$ & $.94(.02)$ & $98.22(1.27)$ \\
\hline Pregnancy Risk (Low) & $1.00(.22)$ & $1.00(.06)$ & $100(.00)$ & $.86(.04)$ & $.93(.03)$ & $96.59(2.05)$ \\
\hline Monetary $(\$ 10)$ & $.36(.82)$ & - & - & $.50(.07)$ & - & - \\
\hline Monetary $(\$ 100)$ & $.70(.69)$ & - & - & $.65(.06)$ & - & - \\
\hline \multicolumn{7}{|c|}{$\begin{array}{l}n . b . \text { AUC = area under the curve; STD = sexually transmitted infection; Raw AUC is calculated individually for each } \\
\text { participant by using the participant data at each time point. AUC is on a } 0-1 \text { scale. An AUC closer to } 0 \text { indicates steeper } \\
\text { discounting or lower preference for delayed condom use; "Immediately Available" Condom preference is based on the } \\
\text { zero-delay response for each condition; Standardized AUC is where the response at each delay is divided by the } \\
\text { immediately available condom response }\end{array}$} \\
\hline
\end{tabular}




\section{Table $3 b$}

Dependent Samples Wilcoxon Signed-ranks Test Median Differences Between and Within Conditions using Raw AUC, Standardized AUC, and "Immediately Available" Condom Preference $(n=27)$

\begin{tabular}{|c|c|c|c|c|c|c|c|}
\hline \multirow[b]{2}{*}{ Variable } & & \multicolumn{2}{|c|}{ Raw $A U C$} & \multicolumn{2}{|c|}{$\begin{array}{c}\text { Standardized } \\
A U C\end{array}$} & \multicolumn{2}{|c|}{$\begin{array}{c}\text { "Immediately } \\
\text { Available" } \\
\text { Condom } \\
\text { Preferences }\end{array}$} \\
\hline & & $Z$ & $p$ & $Z$ & $p$ & $Z$ & $p$ \\
\hline \multirow[t]{7}{*}{ Attraction (High) } & Attraction (Low) & -2.66 & .008 & -2.43 & .015 & -2.29 & .022 \\
\hline & STD Risk (High) & -3.73 & $<.001$ & -3.54 & $<.001$ & -2.67 & .008 \\
\hline & STD Risk (Low) & -1.57 & .117 & -2.07 & .039 & -1.02 & .308 \\
\hline & Pregnancy Risk (High) & -3.25 & .001 & -3.02 & .002 & -2.40 & .017 \\
\hline & Pregnancy Risk (Low) & -3.49 & $<.001$ & -3.58 & $<.001$ & -1.78 & .074 \\
\hline & Monetary $(\$ 10)$ & -0.29 & .775 & & & & \\
\hline & Monetary $(\$ 100)$ & -1.80 & .072 & & & & \\
\hline \multirow[t]{6}{*}{ Attraction (Low) } & STD Risk (High) & -2.28 & .023 & -1.96 & .050 & -1.36 & .173 \\
\hline & STD Risk (Low) & -1.44 & .149 & -0.98 & .326 & -0.73 & .463 \\
\hline & Pregnancy Risk (High) & -0.80 & .422 & -0.39 & .695 & -0.94 & .345 \\
\hline & Pregnancy Risk (Low) & -0.21 & .836 & -0.06 & .955 & -0.14 & .893 \\
\hline & Monetary (\$10) & -2.65 & .008 & & & & \\
\hline & Monetary $(\$ 100)$ & -3.58 & $<.001$ & & & & \\
\hline \multirow{5}{*}{ STD Risk (High) } & STD Risk (Low) & -3.41 & .001 & -2.97 & .003 & -1.90 & .058 \\
\hline & Pregnancy Risk (High) & -2.07 & .038 & -1.82 & .069 & -0.73 & .465 \\
\hline & Pregnancy Risk (Low) & -2.55 & .011 & -2.09 & .037 & -1.44 & .150 \\
\hline & Monetary (\$10) & -3.81 & $<.001$ & & & & \\
\hline & Monetary $(\$ 100)$ & -3.82 & $<.001$ & & & & \\
\hline \multirow[t]{4}{*}{ STD Risk (Low) } & Pregnancy Risk (High) & -2.48 & .013 & -1.93 & .053 & -1.36 & .173 \\
\hline & Pregnancy Risk (Low) & -2.10 & .035 & -2.51 & .012 & -0.49 & .624 \\
\hline & Monetary $(\$ 10)$ & -1.74 & .081 & & & & \\
\hline & Monetary $(\$ 100)$ & -2.91 & .004 & & & & \\
\hline \multirow[t]{3}{*}{ Pregnancy Risk (High) } & Pregnancy Risk (Low) & -1.36 & .173 & 0.00 & 1.00 & -1.15 & .249 \\
\hline & Monetary $(\$ 10)$ & -3.07 & .002 & & & & \\
\hline & Monetary $(\$ 100)$ & -3.82 & $<.001$ & & & & \\
\hline \multirow[t]{2}{*}{ Pregnancy Risk (Low) } & Monetary $(\$ 10)$ & -3.00 & .003 & & & & \\
\hline & Monetary $(\$ 100)$ & -3.91 & $<.001$ & & & & \\
\hline Monetary (\$100) & Monetary $(\$ 10)$ & -2.22 & .026 & & & & \\
\hline
\end{tabular}




\begin{tabular}{|c|c|c|c|c|c|c|c|}
\hline \multicolumn{8}{|c|}{$\begin{array}{l}\text { Table } 4 \mathrm{~b} \\
\text { Spearman Correlation Coefficient with Raw AUC }(n=27)\end{array}$} \\
\hline Variable & 1 & 2 & 3 & 4 & 5 & 6 & 7 \\
\hline 1. Attraction (High) AUC & - & & & & & & \\
\hline 2. Attraction (Low) AUC & .367 & - & & & & & \\
\hline 3. STD Risk (High) AUC & -.081 & .019 & - & & & & \\
\hline 4. STD Risk (Low) AUC & $.642^{* *}$ & .295 & .138 & - & & & \\
\hline 5. Pregnancy Risk (High) AUC & .200 & .325 & .305 & .342 & - & & \\
\hline 6. Pregnancy Risk (Low) AUC & $.431^{*}$ & .265 & .074 & $.696^{* *}$ & $.541^{* *}$ & - & \\
\hline 7. Future as Open & .053 & .140 & -.009 & -.269 & -.263 & -.160 & - \\
\hline 8. Future as Limited & -.111 & -.006 & -.058 & -.195 & -.277 & -.103 & -.119 \\
\hline 9. Future as Ambiguous & -.272 & -.120 & -.105 & -.034 & .105 & .088 & $-.563^{* *}$ \\
\hline 10. MCAS (Pleasure) & -.109 & -.239 & .137 & -.134 & -.208 & -.099 & .248 \\
\hline 11. MCAS (Stigma) & .068 & -.161 & .294 & .269 & .042 & .373 & .146 \\
\hline 12. MCAS (Negotiation and Use) & .048 & .015 & .057 & .056 & .324 & .196 & .023 \\
\hline 13. Sexual Arousal & -.137 & .060 & -.028 & .097 & .010 & .251 & -.378 \\
\hline
\end{tabular}




\begin{tabular}{|c|c|c|c|c|c|c|}
\hline \multicolumn{7}{|c|}{$\begin{array}{l}\text { Table 4b (continued) } \\
\text { Spearman Correlation Coefficient with Raw AUC }(n=27)\end{array}$} \\
\hline Variable & 8 & 9 & 10 & 11 & 12 & 13 \\
\hline \multicolumn{7}{|l|}{ 1. Attraction (High) AUC } \\
\hline \multicolumn{7}{|l|}{ 2. Attraction (Low) AUC } \\
\hline \multicolumn{7}{|l|}{ 3. STD Risk (High) AUC } \\
\hline \multicolumn{7}{|l|}{ 4. STD Risk (Low) AUC } \\
\hline \multicolumn{7}{|l|}{ 5. Pregnancy Risk (High) AUC } \\
\hline \multicolumn{7}{|l|}{ 6. Pregnancy Risk (Low) AUC } \\
\hline \multicolumn{7}{|l|}{ 7. Future as Open } \\
\hline 8. Future as Limited & - & & & & & \\
\hline 9. Future as Ambiguous & .026 & - & & & & \\
\hline 10. MCAS (Pleasure) & .047 & -.381 & - & & & \\
\hline 11. MCAS (Stigma) & .144 & -.309 &. $\mathbf{5 7 0}^{* *}$ & - & & \\
\hline 12. MCAS (Negotiation and Use) & -.264 & -.200 & .226 & $.462^{*}$ & - & \\
\hline 13. Sexual Arousal & .157 & $.488^{* *}$ & -.141 & .036 & .035 & - \\
\hline
\end{tabular}




\begin{tabular}{|c|c|c|c|c|c|c|c|}
\hline \multicolumn{8}{|c|}{$\begin{array}{l}\text { Table } 5 b \\
\text { Spearman Correlation Coefficient with Standardized AUC }(n=27)\end{array}$} \\
\hline Variable & 1 & 2 & 3 & 4 & 5 & 6 & 7 \\
\hline 1. Attraction (High) AUC & - & & & & & & \\
\hline 2. Attraction (Low) AUC & .282 & - & & & & & \\
\hline 3. STD Risk (High) AUC & .077 & .312 & - & & & & \\
\hline 4. STD Risk (Low) AUC & $.621^{* *}$ & .056 & .244 & - & & & \\
\hline 5. Pregnancy Risk (High) AUC & .178 & .334 & .374 & .325 & - & & \\
\hline 6. Pregnancy Risk (Low) AUC & $.440^{*}$ & .001 & .291 & $.771^{* *}$ & $.538^{* *}$ & - & \\
\hline 7. Future as Open & .094 & .181 & -.065 & -.267 & -.222 & -.125 & - \\
\hline 8. Future as Limited & -.062 & .027 & -.152 & -.243 & -.260 & -.150 & -.119 \\
\hline 9. Future as Ambiguous & -.281 & -.115 & -.051 & .010 & .063 & .060 & $-.563^{* *}$ \\
\hline 10. MCAS (Pleasure) & -.081 & -.133 & .228 & -.048 & -.172 & -.085 & .248 \\
\hline 11. MCAS (Stigma) & .075 & -.218 & .178 & .178 & .081 & .282 & .146 \\
\hline 12. MCAS (Negotiation and Use) & -.016 & .037 & .089 & -.056 & .284 & .038 & .023 \\
\hline 13. Sexual Arousal & -.185 & -.034 & .089 & .115 & -.038 & .205 & -.378 \\
\hline
\end{tabular}




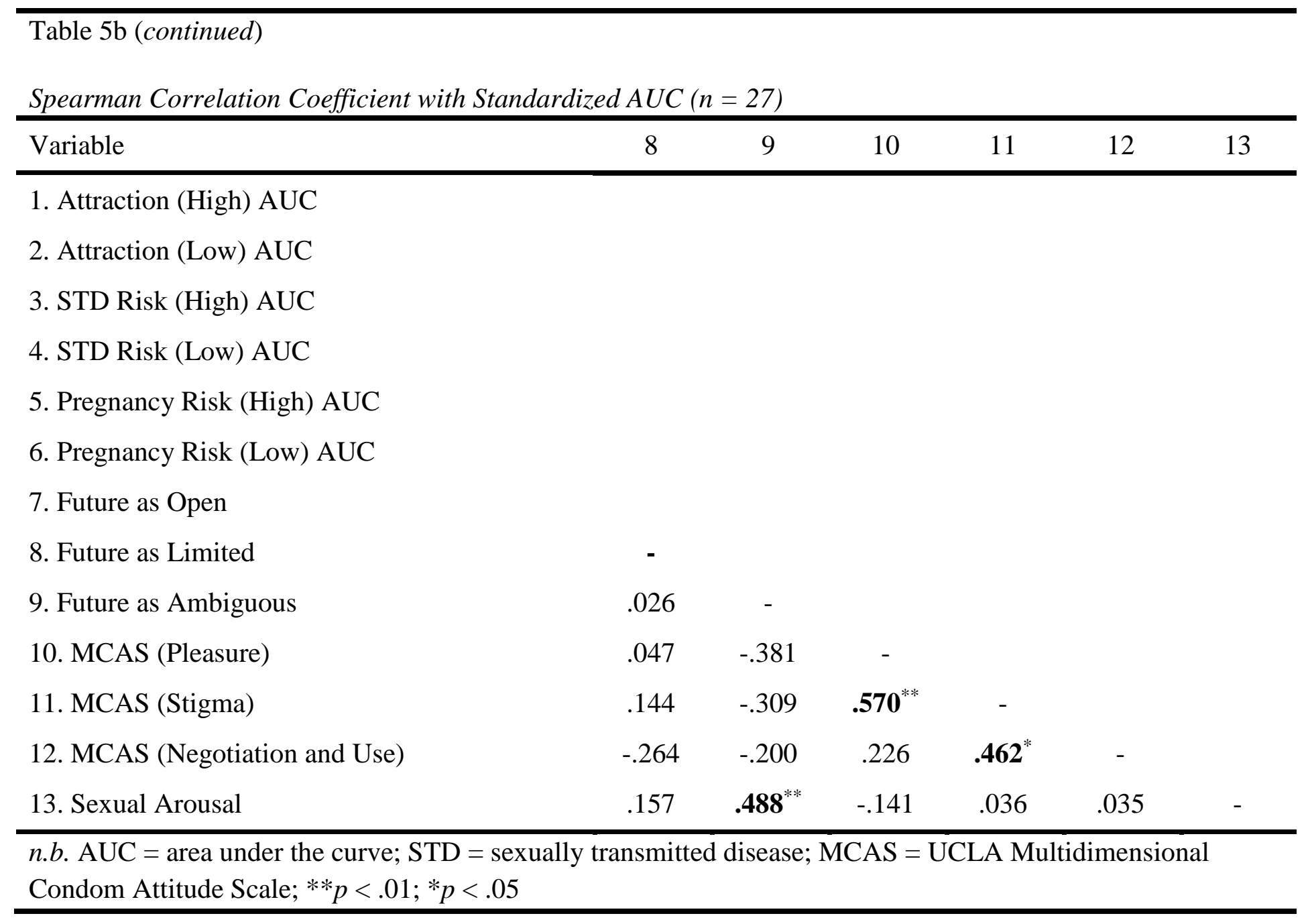




\begin{tabular}{|c|c|c|c|c|c|c|c|}
\hline \multicolumn{8}{|l|}{ Table $6 \mathrm{~b}$} \\
\hline Variable & 1 & 2 & 3 & 4 & 5 & 6 & 7 \\
\hline 1. Attraction (High) & - & & & & & & \\
\hline 2. Attraction (Low) & $.613^{* *}$ & - & & & & & \\
\hline 3. STD Risk (High) & -.209 & -.117 & - & & & & \\
\hline 4. STD Risk (Low) & .315 & $.387^{*}$ & .213 & - & & & \\
\hline 5. Pregnancy Risk (High) & $.394^{*}$ & .307 & -.080 & .253 & - & & \\
\hline 6. Pregnancy Risk (Low) & $.410^{*}$ & $.613^{* *}$ & -.134 & .085 & .266 & - & \\
\hline 7. Future as Open & -.104 & .019 & .042 & -.098 & -.287 & -.115 & - \\
\hline 8. Future as Limited & -.228 & -.119 & .049 & -.171 & -.245 & -.098 & -.119 \\
\hline 9. Future as Ambiguous & -.170 & -.150 & -.090 & -.149 & .213 & .255 & $-.563^{* *}$ \\
\hline 10. MCAS (Pleasure) & -.165 & -.249 & -.012 & -.041 & -.332 & -.208 & .248 \\
\hline 11. MCAS (Stigma) & -.113 & .269 & .225 & .206 & -.166 & .073 & .146 \\
\hline 12. MCAS (Negotiation and Use) & .174 & .238 & .001 & .122 & $.395^{*}$ & .083 & .023 \\
\hline 13. Sexual Arousal & .032 & .016 & -.106 & -.184 & .146 & .226 & -.378 \\
\hline
\end{tabular}




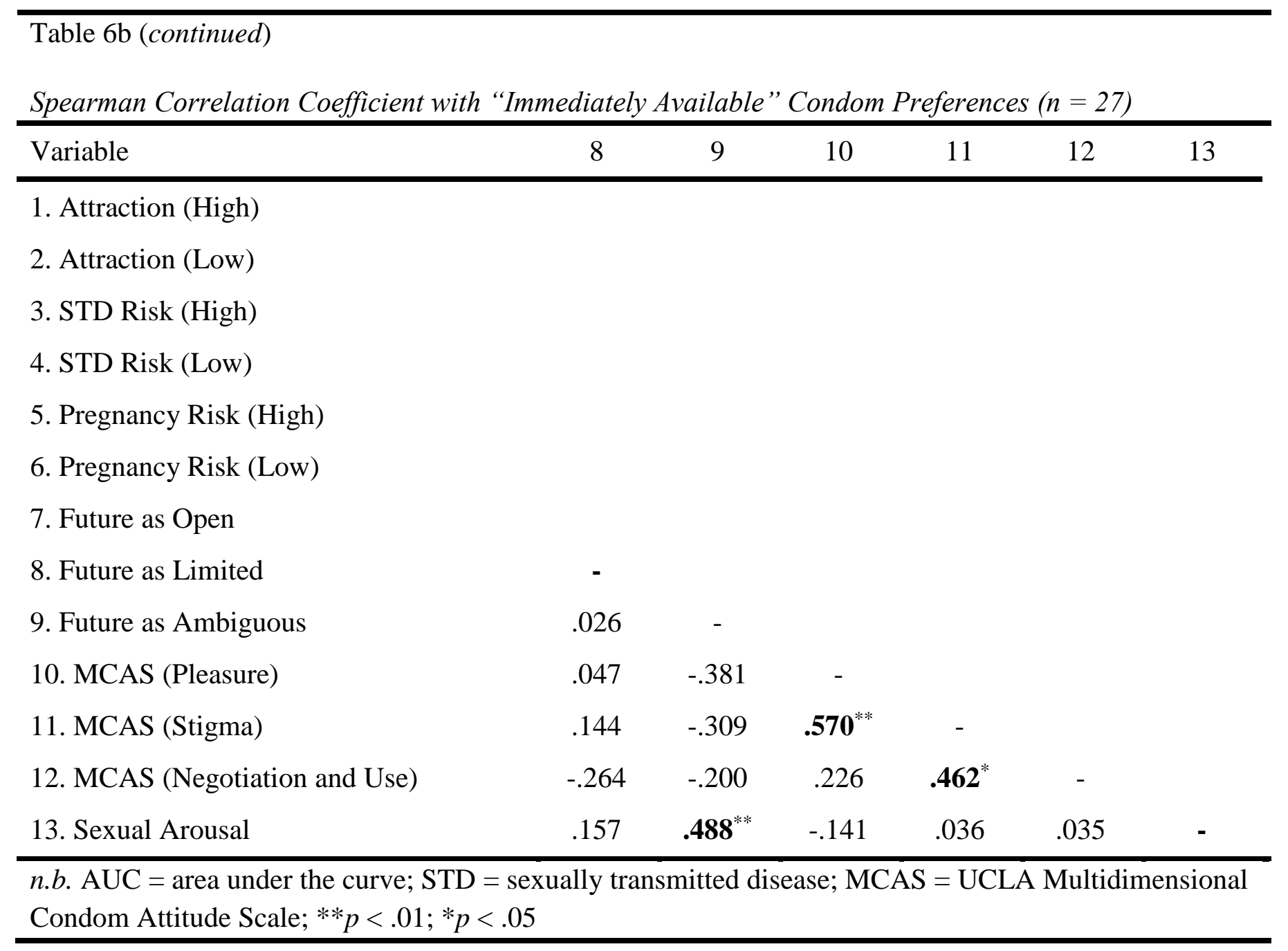




\begin{tabular}{|c|c|c|c|c|c|}
\hline \multicolumn{6}{|l|}{ Table $7 \mathrm{~b}$} \\
\hline & $\begin{array}{l}\text { Women } \\
n=21\end{array}$ & $\begin{array}{l}\text { Men } \\
n=6\end{array}$ & Overall & \multirow[b]{2}{*}{$U$} & \multirow[b]{2}{*}{$p$} \\
\hline Variable & & Median & & & \\
\hline \multicolumn{6}{|l|}{ Raw AUC } \\
\hline Attraction (High) & $.69(.82)$ & $.89(.64)$ & $.78(.69)$ & 60.0 & .860 \\
\hline Attraction (Low) & $1.00(.26)$ & $1.00(.06)$ & $1.00(.19)$ & 46.0 & .253 \\
\hline STD Risk (High) & $1.00(.00)$ & $1.00(.00)$ & $1.00(.00)$ & 59.5 & .708 \\
\hline STD Risk (Low) & $.92(.44)$ & $.92(.43)$ & $.92(.40)$ & 59.0 & .807 \\
\hline Pregnancy Risk (High) & $1.00(.20)$ & $1.00(.04)$ & $1.00(.12)$ & 51.0 & .386 \\
\hline Pregnancy Risk (Low) & $1.00(.34)$ & $1.00(.04)$ & $1.00(.22)$ & 46.0 & .276 \\
\hline \multicolumn{6}{|l|}{ Standardized AUC } \\
\hline Attraction (High) & $.80(.61)$ & $.99(.56)$ & $.85(.43)$ & 62.5 & .976 \\
\hline Attraction (Low) & $1.00(.14)$ & $1.00(.01)$ & $1.00(.01)$ & 51.0 & .386 \\
\hline STD Risk (High) & $1.00(.00)$ & $1.00(.00)$ & $1.00(.00)$ & 60.0 & .593 \\
\hline STD Risk (Low) & $1.00(.26)$ & $.98(.28)$ & $1.00(.17)$ & 57.0 & .706 \\
\hline Pregnancy Risk (High) & $1.00(.06)$ & $1.00(.04)$ & $1.00(.03)$ & 53.0 & .470 \\
\hline Pregnancy Risk (Low) & $1.00(.13)$ & $1.00(.03)$ & $1.00(.06)$ & 52.0 & .459 \\
\hline \multicolumn{6}{|c|}{ "Immediately Available" Condom Preferences (\%) } \\
\hline Attraction (High) & $100(12.5)$ & $100(20.00)$ & $100(13.0)$ & 60.0 & .840 \\
\hline Attraction (Low) & $100(.00)$ & $100(4.25)$ & $100(.00)$ & 62.0 & .925 \\
\hline STD Risk (High) & $100(.00)$ & $100(0.25)$ & $100(.00)$ & 56.0 & .369 \\
\hline STD Risk (Low) & $100(.00)$ & $100(30.25)$ & $100(.00)$ & 49.5 & .245 \\
\hline Pregnancy Risk (High) & $100(.00)$ & $100(.00)$ & $100(.00)$ & 57.0 & .441 \\
\hline Pregnancy Risk (Low) & $100(.50)$ & $100(.00)$ & $100(.00)$ & 48.0 & .197 \\
\hline
\end{tabular}




\begin{tabular}{|c|c|c|c|c|c|c|}
\hline $\begin{array}{l}\text { Table } 8 \mathrm{~b} \\
\text { Mann-Whitney U Comparison }\end{array}$ & Participants & ith Imputed L & ta to those 1 & Complete 1 & & \\
\hline & $\begin{array}{c}\text { Complete } \\
\text { Discounting } \\
\text { Data } \\
(n=27) \\
\end{array}$ & $\begin{array}{c}\text { Imputed } \\
\text { Discounting } \\
\text { Data } \\
(n=86)\end{array}$ & $\begin{array}{c}\text { Complete } \\
\text { Discounting } \\
\text { Data } \\
(n=27) \\
\end{array}$ & $\begin{array}{c}\text { Imputed } \\
\text { Discounting } \\
\text { Data } \\
(n=86)\end{array}$ & & \\
\hline Variable & Medial & $(I Q R)$ & Mear & $(S E)$ & $U$ & $p$ \\
\hline Raw AUC & & & & & & \\
\hline Attraction (High) & $.78(.69)$ & $.38(.82)$ & $.63(.07)$ & $.43(.04)$ & 750.5 & .011 \\
\hline Attraction (Low) & $1.00(.19)$ & $.53(.81)$ & $.86(.05)$ & $.55(.04)$ & 525.7 & .002 \\
\hline STD Risk (High) & $1.00(.00)$ & $.98(.67)$ & $.98(.02)$ & $.69(.04)$ & 569.2 & $<.001$ \\
\hline STD Risk (Low) & $.92(.40)$ & $.35(.82)$ & $.77(.06)$ & $.44(.04)$ & 563.8 & $<.001$ \\
\hline Pregnancy Risk (High) & $1.00(.12)$ & $.92(.64)$ & $.91(.03)$ & $.69(.04)$ & 686.0 & .002 \\
\hline Pregnancy Risk (Low) & $1.00(.22)$ & $.54(.80)$ & $.86(.04)$ & $.53(.04)$ & 494.7 & $<.001$ \\
\hline Monetary (\$10) & $.36(.82)$ & $.31(.64)$ & $.50(.07)$ & $.44(.04)$ & 984.8 & .431 \\
\hline Monetary (\$100) & $.70(.69)$ & $.59(.59)$ & $.65(.06)$ & $.57(.04)$ & 982.7 & .381 \\
\hline Standardized & & & & & & \\
\hline Attraction (High) & $.85(.43)$ & $.54(.79)$ & $.71(.07)$ & $.56(.04)$ & 863.5 & .196 \\
\hline Attraction (Low) & $1.00(.01)$ & $.68(.79)$ & $.89(.05)$ & $.60(.05)$ & 659.5 & .077 \\
\hline STD Risk (High) & $1.00(.00)$ & $1.00(.83)$ & $.98(.02)$ & $.72(.04)$ & 672.0 & .006 \\
\hline STD Risk (Low) & $1.00(.17)$ & $.54(.79)$ & $.85(.05)$ & $.58(.05)$ & 664.2 & .069 \\
\hline Pregnancy Risk (High) & $1.00(.03)$ & $1.00(.57)$ & $.94(.02)$ & $.76(.04)$ & 832.3 & .073 \\
\hline Pregnancy Risk (Low) & $1.00(.06)$ & $.97(.67)$ & $.93(.03)$ & $.71(.04)$ & 783.8 & .079 \\
\hline "Immediately Available" Cond & m Preferences & $(\%)$ & & & & \\
\hline Attraction (High) & $100(13)$ & $69(98.00)$ & $91.3(2.79)$ & $57.9(4.5)$ & 619.5 & $<.001$ \\
\hline Attraction (Low) & $100(0)$ & $77.5(53.00)$ & $96.5(2.01)$ & $66.2(3.9)$ & 529.0 & $<.001$ \\
\hline STD Risk (High) & $100(0)$ & $100(49.25)$ & $99.6(0.33)$ & $73.9(3.9)$ & 669.5 & $<.001$ \\
\hline STD Risk (Low) & $100(0)$ & $52(75.25)$ & $94.8(2.62)$ & $55.9(4.0)$ & 419.5 & $<.001$ \\
\hline Pregnancy Risk (High) & $100(0)$ & $100(34.00)$ & $98.2(1.27)$ & $79.0(3.6)$ & 696.5 & $<.001$ \\
\hline Pregnancy Risk (Low) & $100(0)$ & $79(81.50)$ & $96.6(2.05)$ & $62.3(4.3)$ & 509.0 & $<.001$ \\
\hline
\end{tabular}




\section{Figure 1b.}

Percent of Immediate Value Chosen by Delay Using Complete Data $(n=27)$

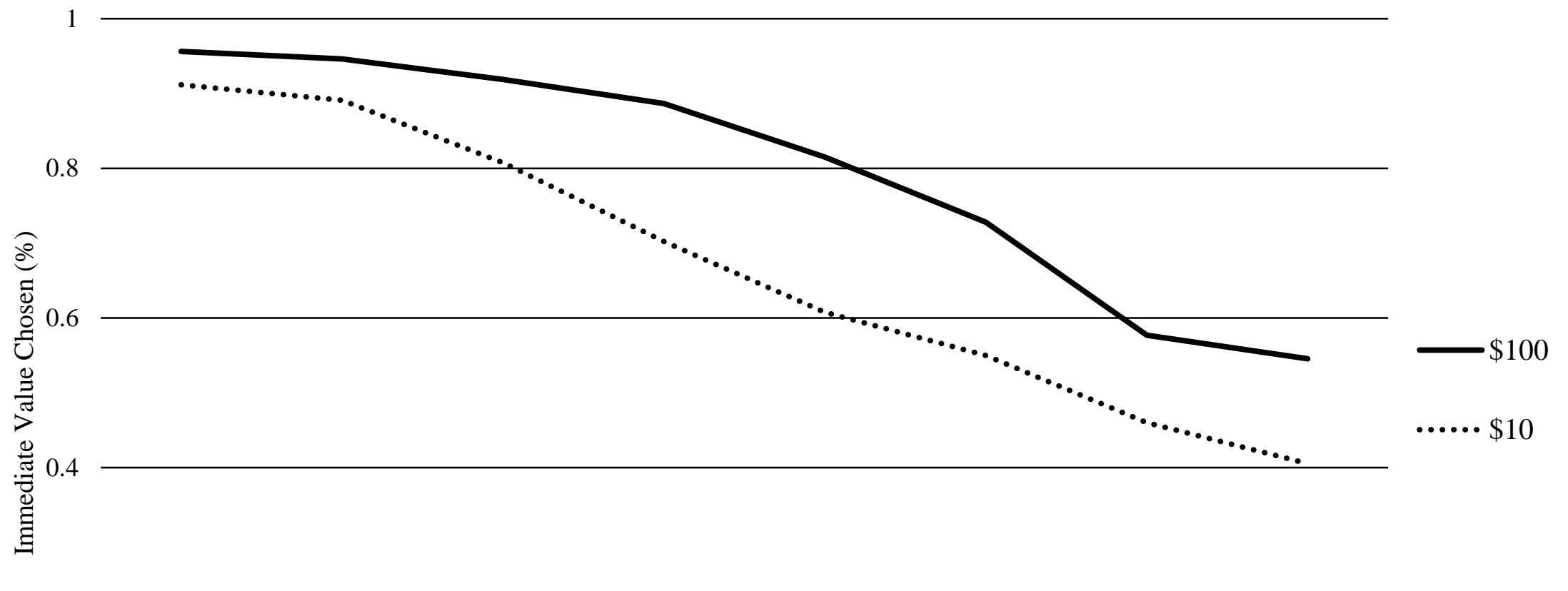

0.2

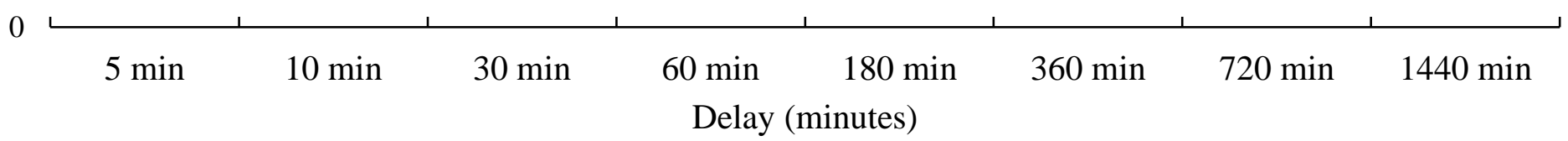




\section{Figure 2b.}

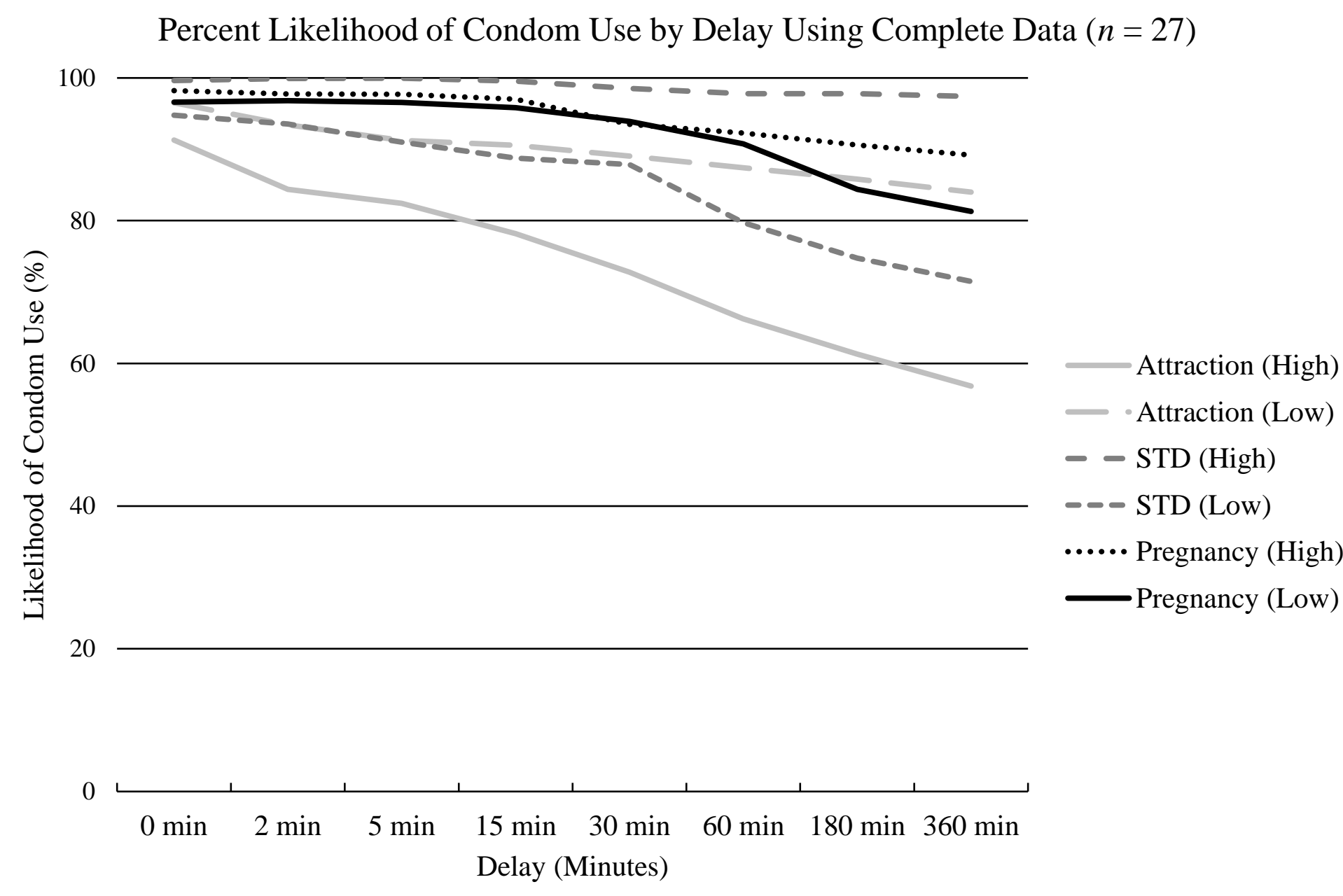




\section{Figure 3b.}

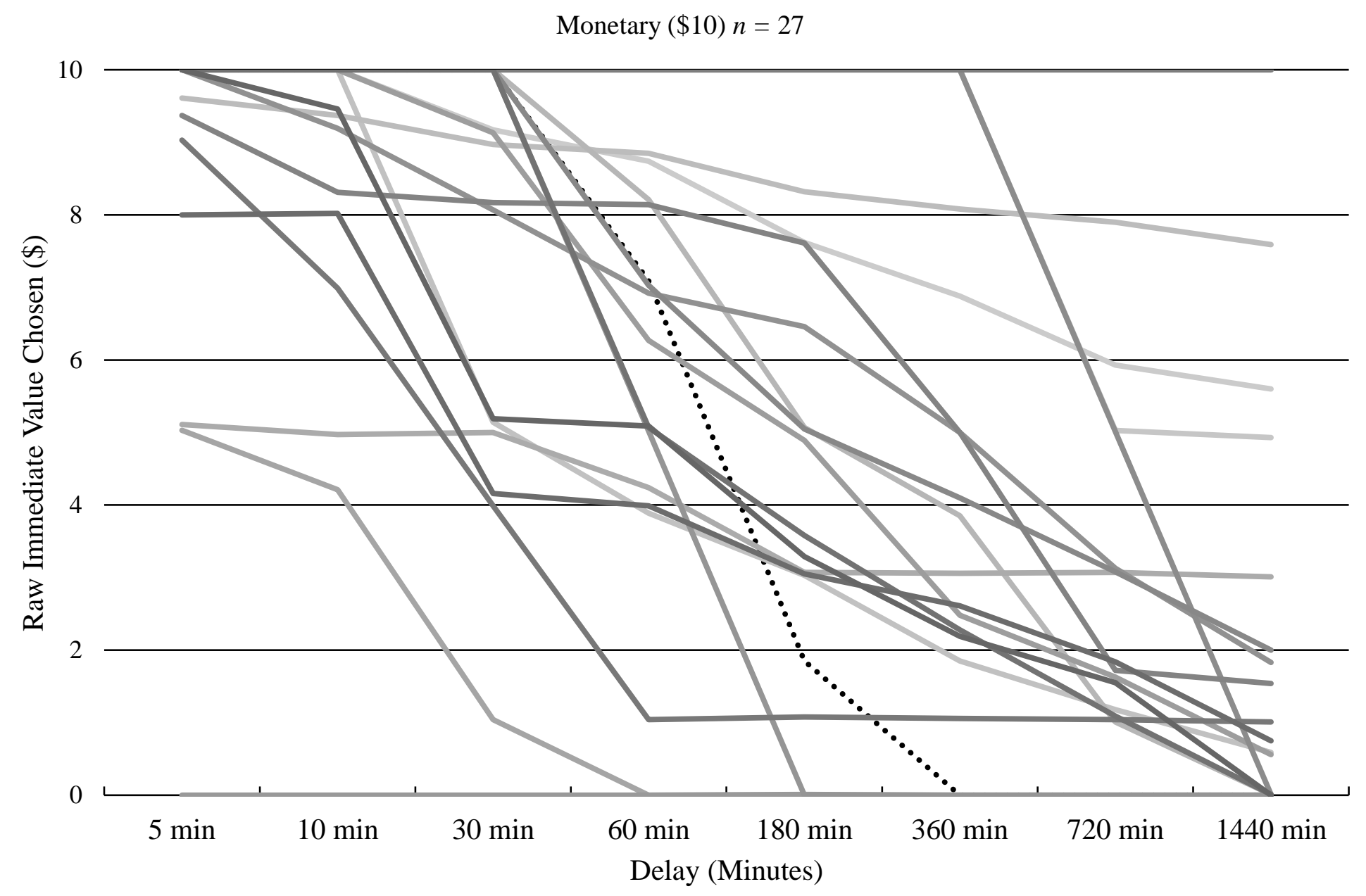




\section{Figure 4b.}

Monetary $(\$ 100) n=27$

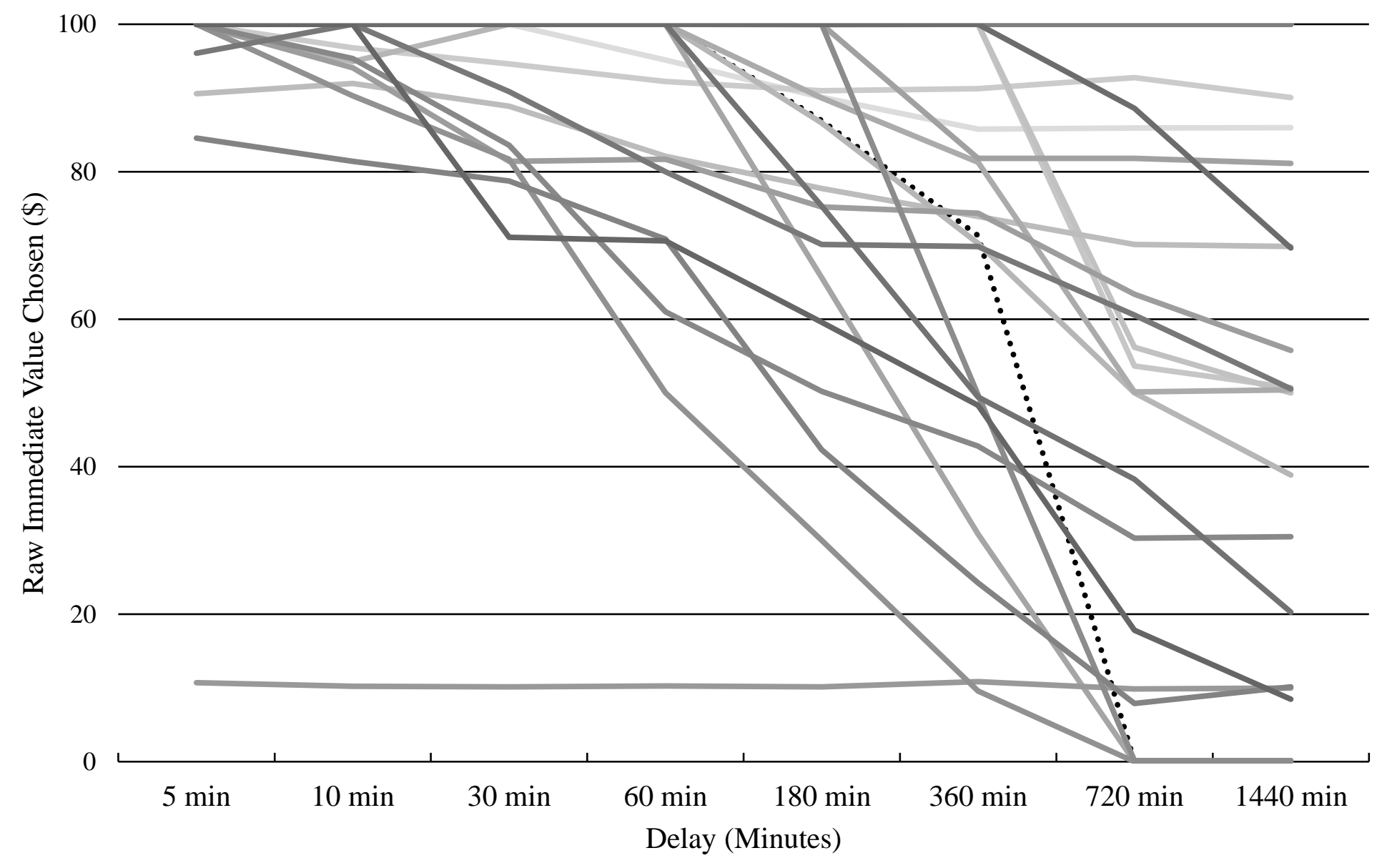




\section{Figure 5b.}

High Attractiveness $n=27$

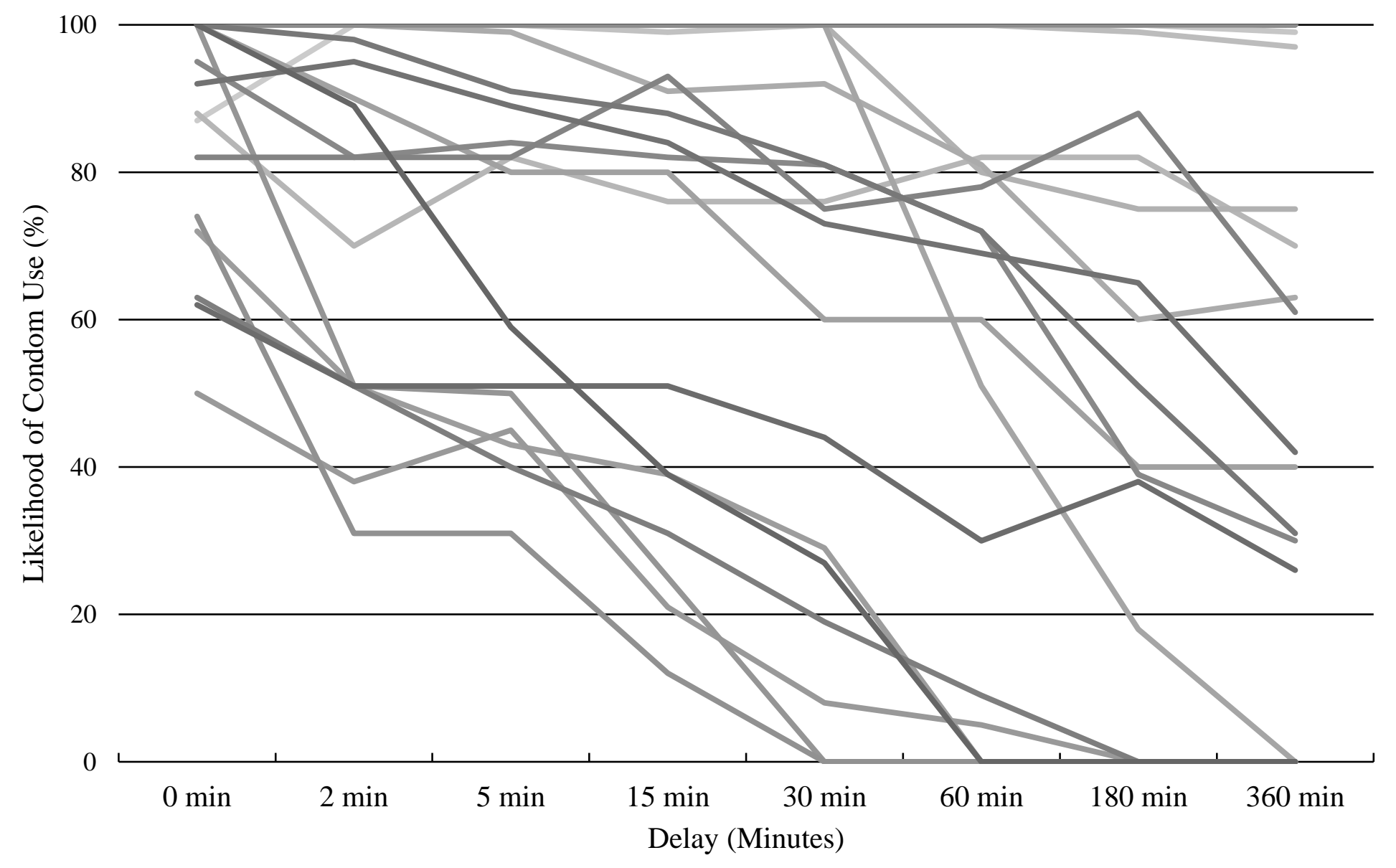




\section{Figure $6 b$.}

Low Attractiveness $n=27$

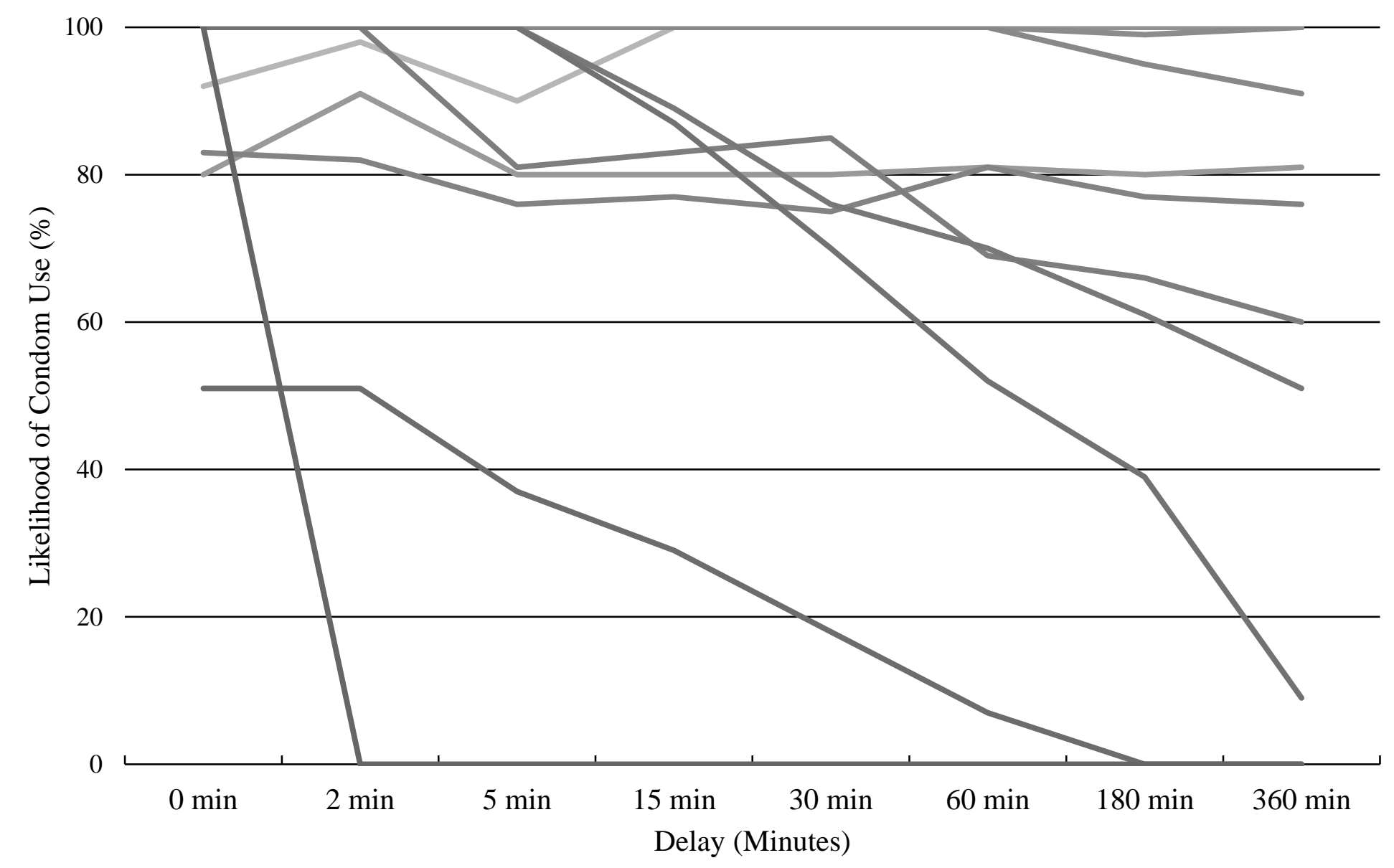




\section{Figure $7 b$.}

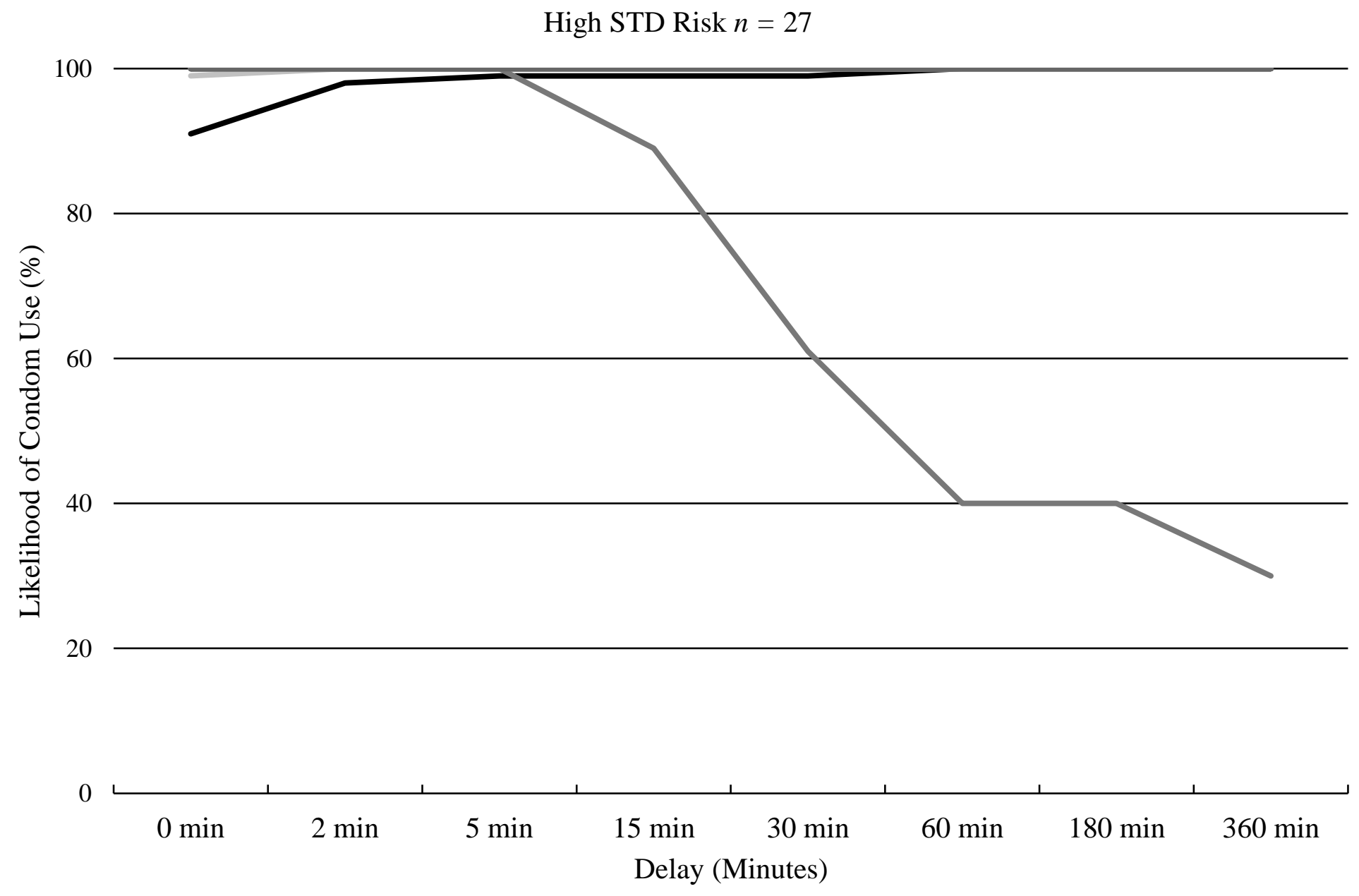




\section{Figure 8b.}

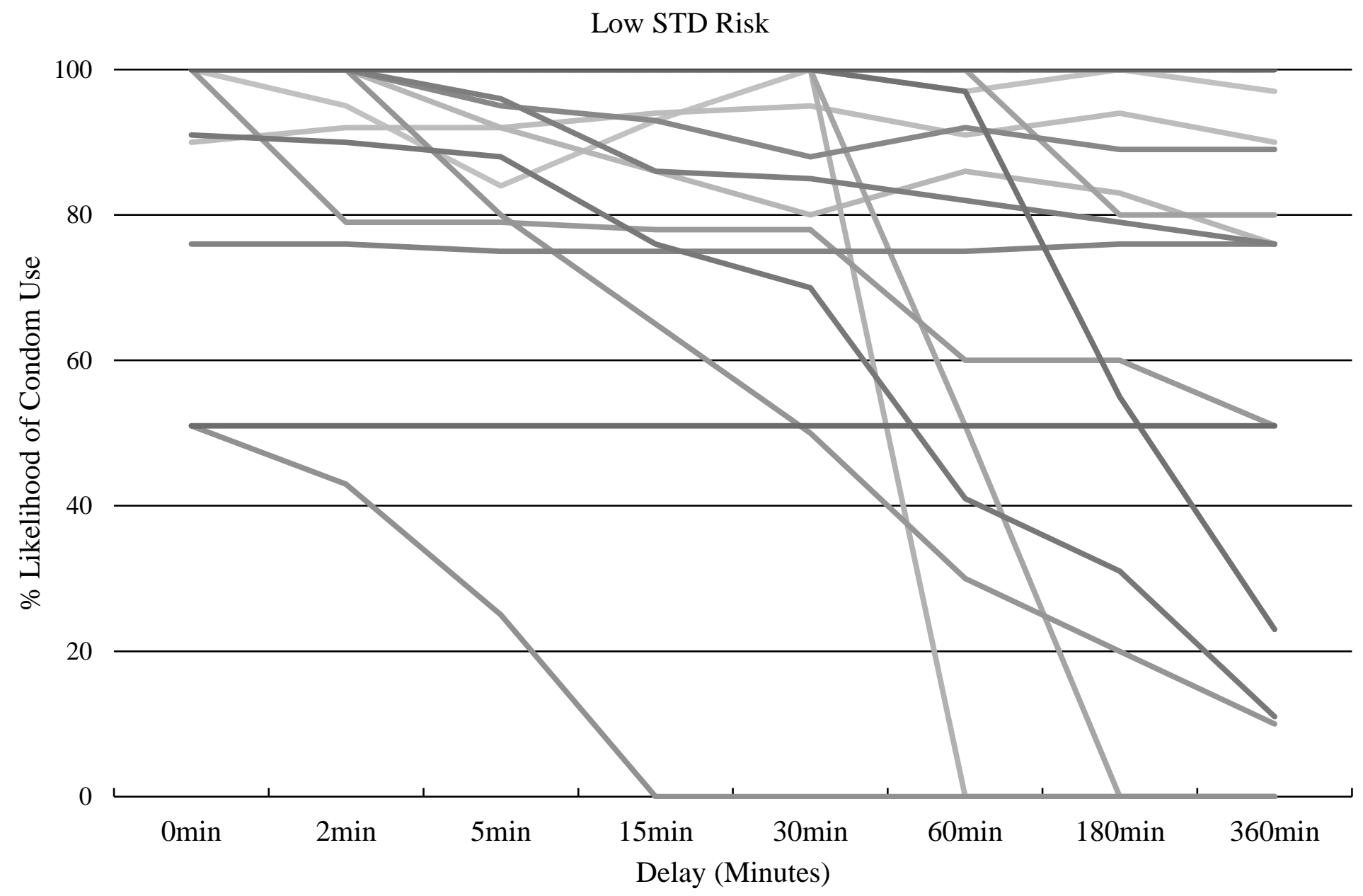




\section{Figure 9b.}

High Pregnancy Risk $n=27$

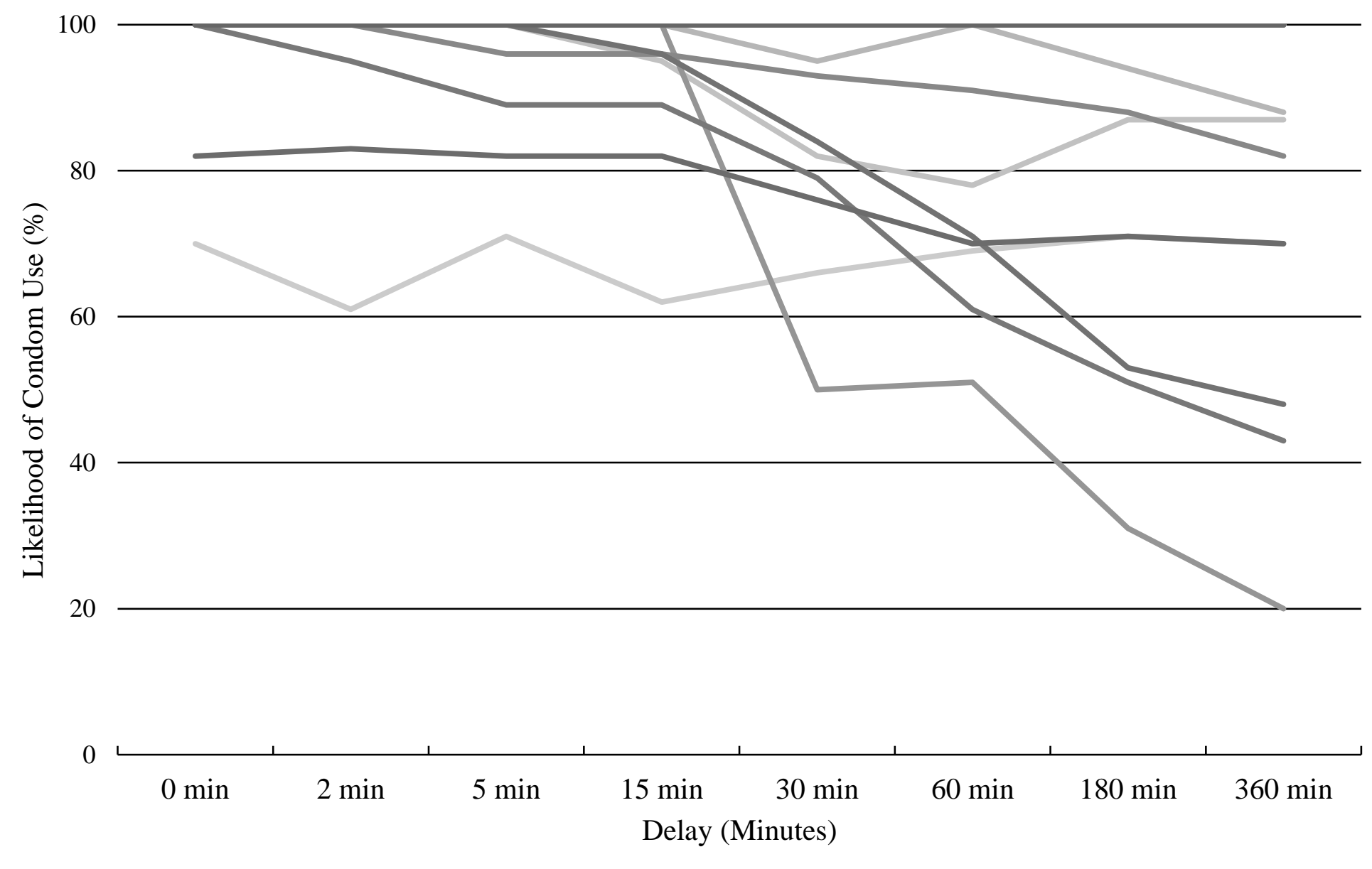




\section{Figure 10b.}

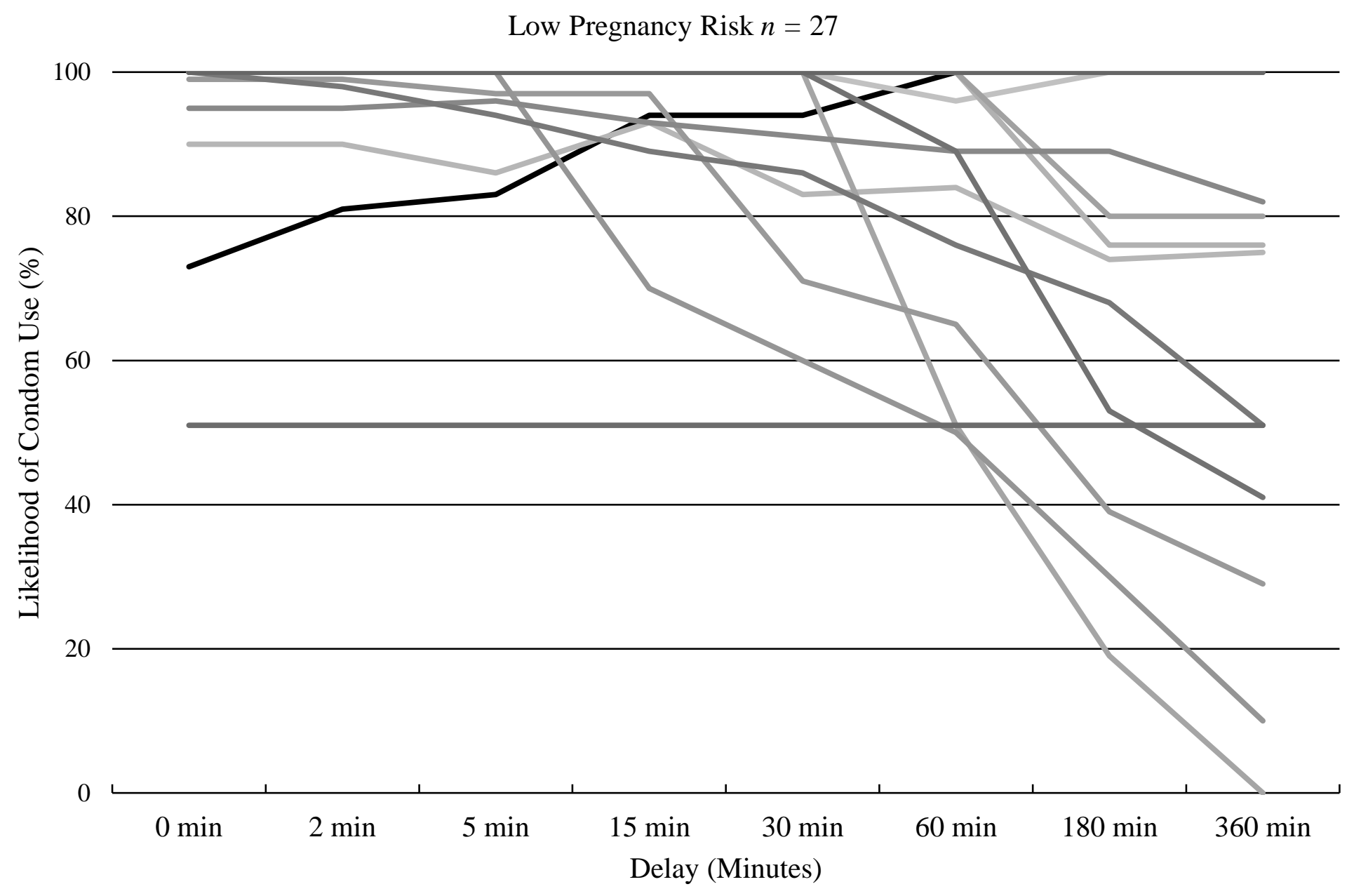




\section{Figure $11 b$.}

Incomplete and Complete Data (Monetary)

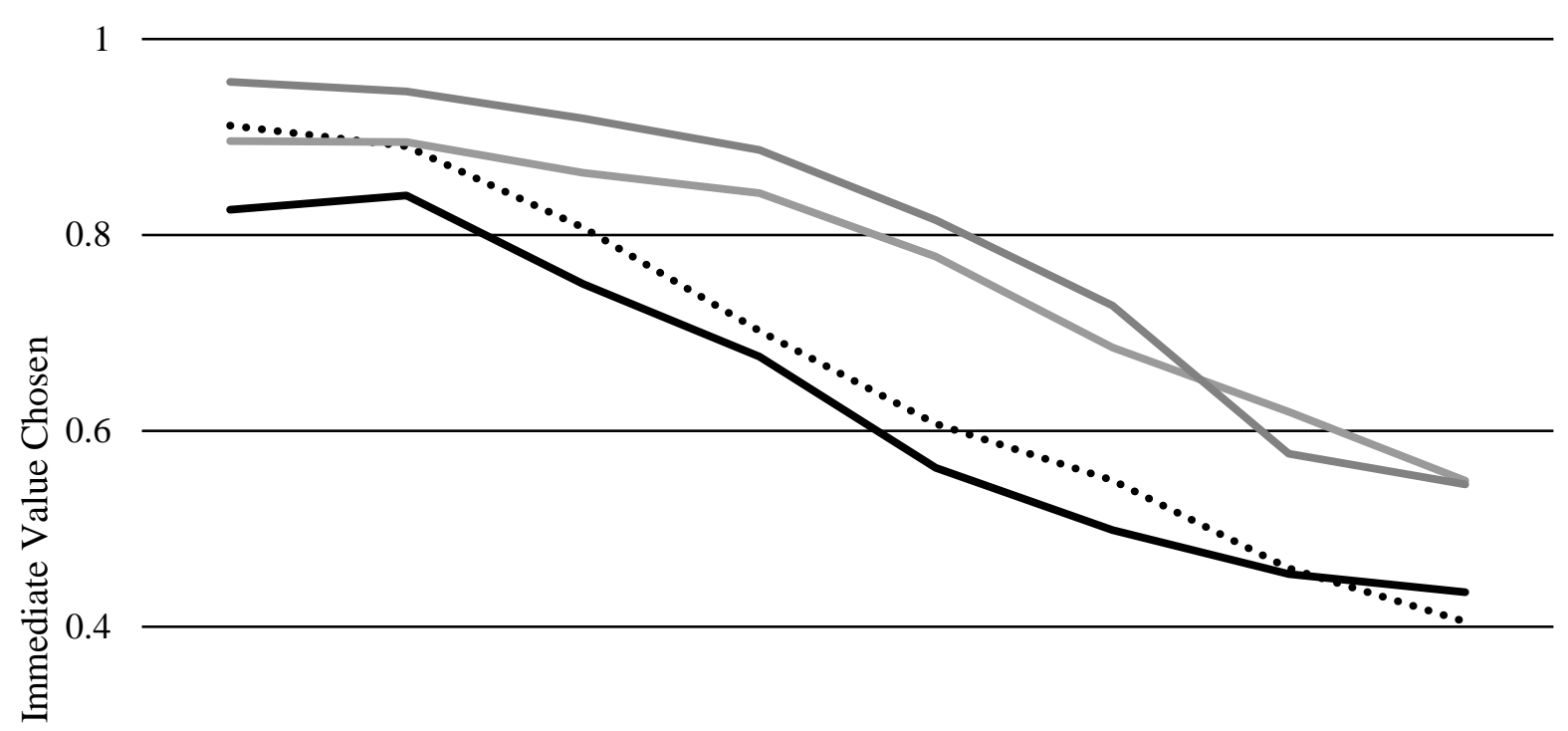

$\longrightarrow \$ 10-$ Incomplete ......\$10 Complete $\longrightarrow \$ 100$ Incomplete $\longrightarrow \$ 100$ Complete

0.2

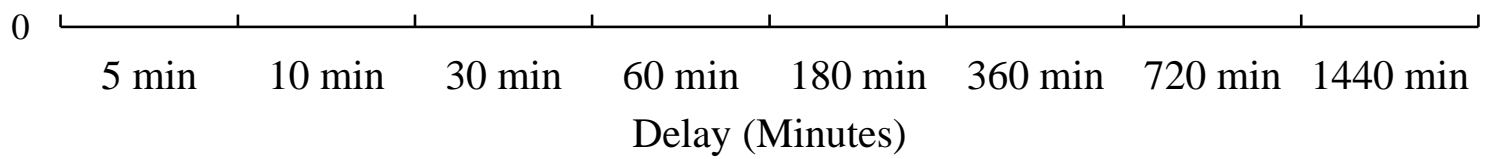




\section{Figure 12b.}

Incomplete and Complete Data (Attraction)

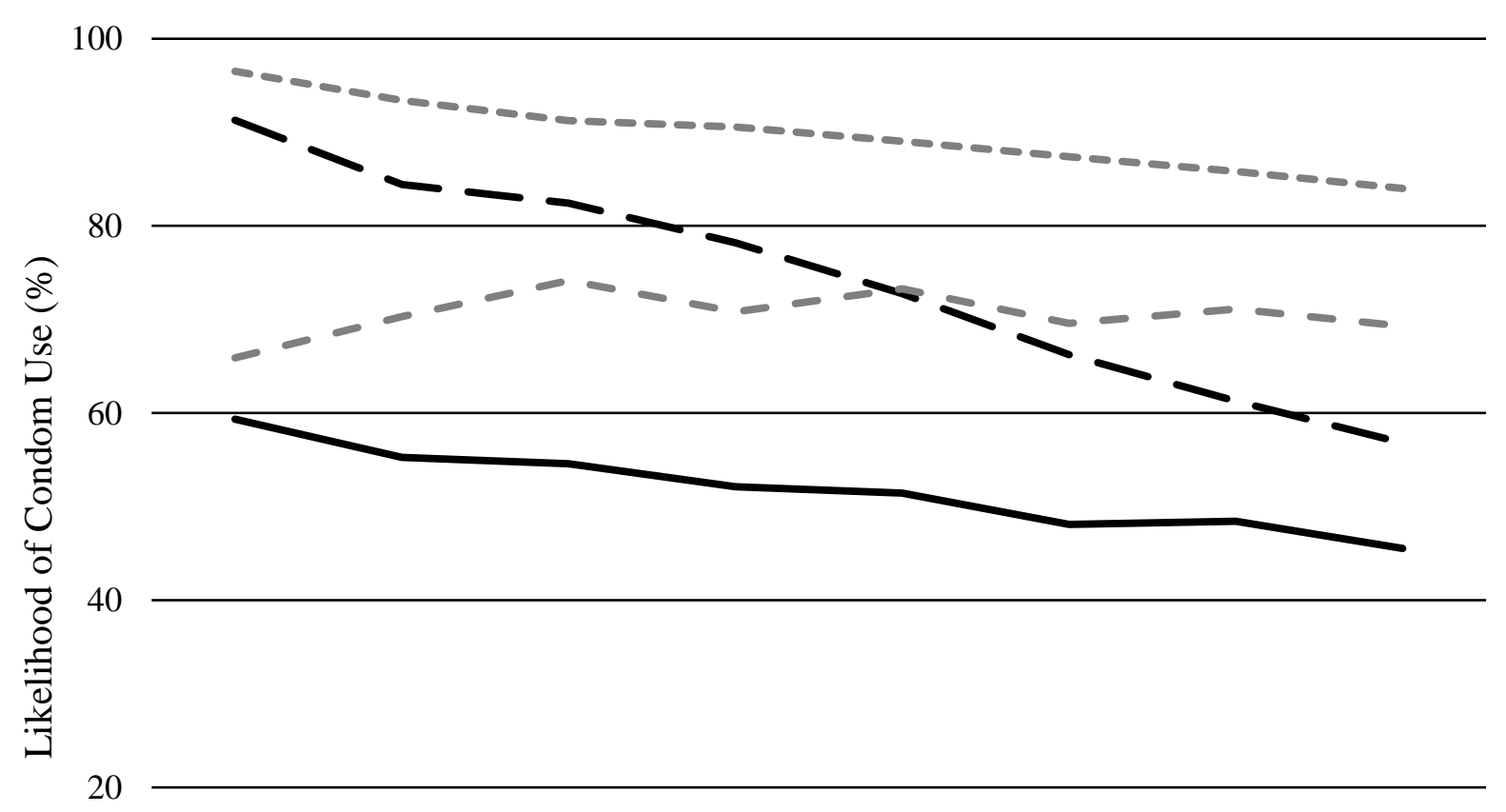

Attraction (High) Incomplete - Attraction (High) Complete - Attraction (Low) Incomplete

\begin{tabular}{|c|c|c|c|c|c|c|}
\hline $0 \mathrm{~min}$ & $2 \min$ & $5 \mathrm{~min}$ & $\begin{array}{l}15 \text { min } \\
\text { Delay }\end{array}$ & $\begin{array}{l}30 \mathrm{~min} \\
\text { Minutes) }\end{array}$ & $60 \mathrm{~min}$ & $180 \mathrm{~min} 360 \mathrm{~min}$ \\
\hline
\end{tabular}




\section{Figure 13b.}

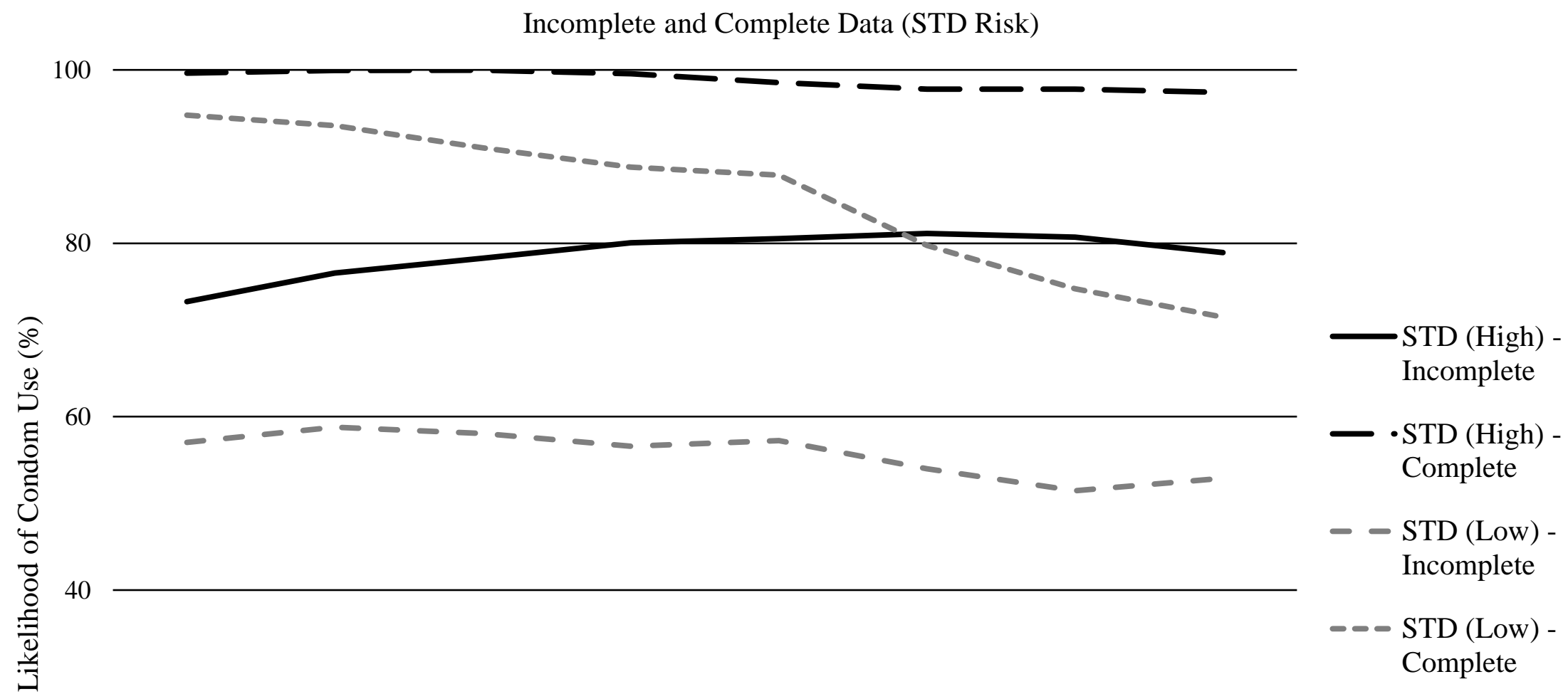

20

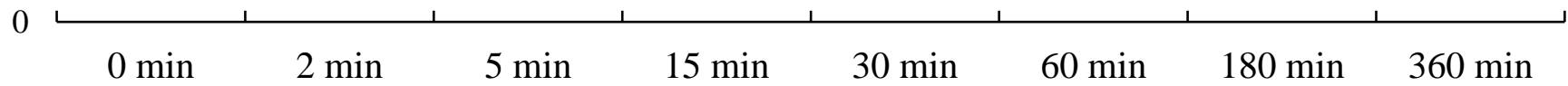

Delay (Minutes) 
Figure 14b.

Incomplete and Complete Data (Pregnancy Risk)

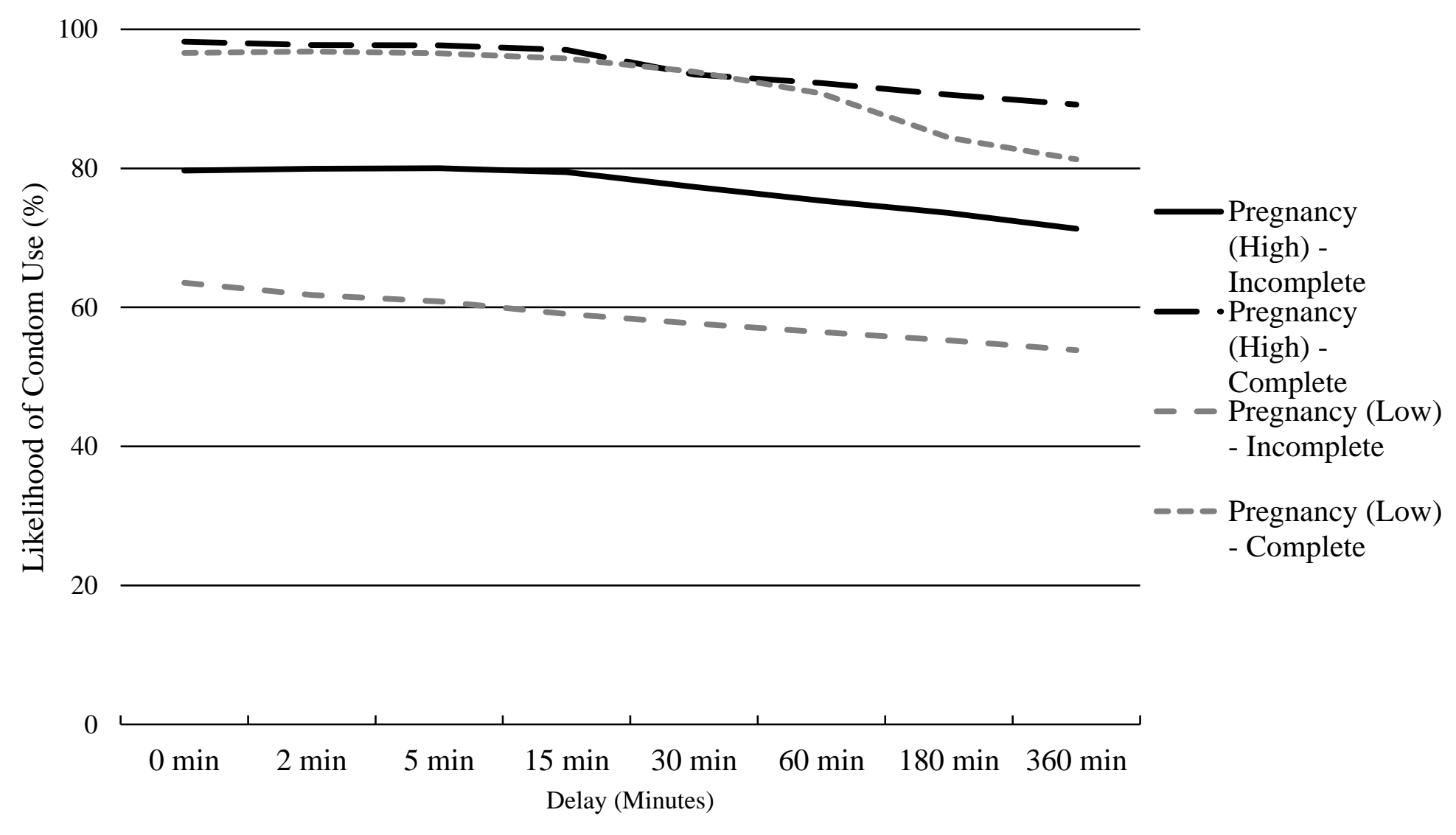




\section{Appendix F}

A series of one sample $t$-tests were used to compare a sample of non-treatment seeking cocaine dependent individuals (Johnson \& Bruner, 2012) mean AUC and our sample mean AUC. Few other sexual discounting studies included descriptives for the Sexual Discounting Task. No studies that used ecologically valid delays for the Sexual Discounting Task included descriptives of AUC or "Immediately Available" Condom Preferences. Comparisons of with the Pregnancy Risk condition are not available due to the novelty of this task.

Participants in the analytic sample did not significantly differ on AUC in the High Attraction condition $\left(M_{A U C}=.47\right)$ compared to the Johnson and Bruner $(2012)$ sample $\left(M_{A U C}=\right.$ $.42) ; t(112)=1.91, p=.156$. Participants in the analytic sample did not significantly differ on AUC in the Low Attraction condition $\left(M_{A U C}=.62\right)$ compared to the Johnson and Bruner $(2012)$ sample $\left(M_{A U C}=.61\right) ; t(112)=1.33, p=.749$. Additionally, participants in the analytic sample did not significantly differ on AUC in the High STD Risk condition $\left(M_{A U C}=.76\right)$ compared to the Johnson and Bruner $(2012)$ sample $\left(M_{A U C}=.75\right) ; t(112)=1.20, p=.721$. Lastly, participants in the analytic sample had significantly higher AUC in the Low STD Risk condition $\left(M_{A U C}=\right.$ $.52)$ compared to the Johnson and Bruner (2012) sample $\left(M_{A U C}=.44\right) ; t(112)=2.71, p=.044$.

When comparing "Immediately Available" Condom Preference, different patterns emerged. Participants in the analytic sample significantly differed on immediately available condom preference in the High Attraction condition $(M=65.9 \%)$ compared to the Johnson and Bruner (2012) sample $(M=75.4 \%) ; t(112)=-2.55, p=.012$. Participants in the analytic sample significantly differed on immediately available condom preference in the Low Attraction condition $(M=73.4 \%)$ compared to the Johnson and Bruner (2012) sample $(M=85.6 \%) ; t(112)$ $=-3.76, p<.001$. Additionally, participants in the analytic sample significantly differed on 
immediately available condom preference in the High STD Risk condition $(M=80.0 \%)$ compared to the Johnson and Bruner (2012) sample $(M=96.9 \%) ; t(112)=-5.36, p<.001$. Lastly, participants in the analytic sample were not significantly different on immediately available condom preference in the Low STD Risk condition $(M=65.2 \%)$ compared to the Johnson and Bruner (2012) sample $(M=71.7 \%) ; t(112)=-1.87, p=.065$.

A series of one sample $t$-tests were used to compare a sample of not-in-treatment individuals who reported heroin and cocaine use MCAS subscale means and our sample means. Participants in the analytic sample had significantly lower MCAS Condom Pleasure $(M=16.2)$ compared to the Rosengard et al. (2006) sample $(M=18.7) ; t(111)=-4.71, p<.001$. Additionally, participants in the analytic sample did not significantly differ on MCAS Identity Stigma Related to Condom Use $(M=28.2)$ compare to the Rosengard et al. (2006) sample $(M=$ $28.5) ; t(112)=-.45, p=.657$. Lastly, participants in the analytic sample did not significantly differ on MCAS Embarrassment About Negotiation and Use of Condoms $(M=27.1)$ compare to the Rosengard et al. (2006) sample $(M=26.1) ; t(111)=1.73, p=.086$.

A series of one sample $t$-tests were used to compare a healthy adult sample Future Time Perspective subscale means and our sample means. Participants in the analytic sample had slightly lower perception of the Future as Open $(M=15.7)$ compared to the Brothers et al. (2014) sample $(M=16.6) ; t(112)=-3.22, p=.002$. Additionally, participants in the analytic sample did not significantly differ on the perception of the Future as Limited $(M=12.2)$ compared to the Brothers et al. (2014) sample $(M=12.3) ; t(112)=-.20, p=.843$. Lastly, participants in the analytic sample had significantly higher perception of the Future as Ambiguous $(M=11.8)$ compared to the Brothers et al. (2014) sample $(M=10.0)$; t(112) $=5.30, p<.001$. 